\title{
Applied Stochastic Control in High Frequency and Algorithmic Trading
}

by

\author{
Jason Ricci
}

A thesis submitted in conformity with the requirements

for the degree of Doctor of Philosophy Graduate Department of Statistical Sciences University of Toronto

(C) Copyright 2014 by Jason Ricci 


\begin{abstract}
Applied Stochastic Control in High Frequency and Algorithmic Trading

Jason Ricci

Doctor of Philosophy

Graduate Department of Statistical Sciences

University of Toronto
\end{abstract}

2014

In this thesis, problems in the realm of high frequency trading and optimal market making are established and solved in both single asset and multiple asset economies. For an agent that is averse to holding large inventories for long periods of time, optimal high frequency trading strategies are derived via stochastic control theory and solving the corresponding Hamilton-Jacobi-Bellman equations. These strategies are analyzed and it is shown that both inventory control and accounting for adverse selection play critical roles in the success of an algorithmic trading strategy.

In the single asset problem, a market maker actively modifies her limit quotes in an economy with asymmetric information. She attempts to keep her inventory small and posts her limit orders in the limit order book at a depth that mitigates her adverse selection risk, while not posting too deep in the book as to generate no trade flow. In addition to this behaviour, a profit maximizing investor trading in multiple assets also seeks out statistical arbitrage opportunities and acts aggressively via the submission of market orders when it is deemed optimal to do so.

Throughout this thesis, numerical and practical considerations are made a priority. Full scale calibration and estimation methods are given in detail, as well as dimensional reductions for large scale numerical procedures, where appropriate. The bridge from abstract mathematical theory to practical real-time implementation is made complete as an entire chapter is dedicated to applications on real data. 


\section{Acknowledgements}

First and foremost, I would like to express my deepest gratitude to my thesis advisor, Sebastian Jaimungal, who has provided an unparallelled level of guidance and support throughout these past few years. This thesis would not have been possible without his insight and excellence in the discipline.

I would also like to thank my coauthor, Álvaro Cartea from University College London, for always being willing to share his ideas and advice during our collaboration.

Furthermore, I am grateful to Jeffrey Rosenthal, Luis Seco, and Bálint Virág for taking the time to be part of my thesis committee and providing valuable input throughout various stages of my research, as well as Mark Reesor from the University of Western Ontario for having been my external examiner.

I would also like to thank the graduate administrator of the Department of Statistical

Sciences, Andrea Carter, for her unwavering patience when I had inquiries regarding administrative matters.

Finally, I gratefully acknowledge the financial support of Bank of Montreal (BMO) Capital Markets and the Ontario Graduate Scholarship (OGS). 


\section{Contents}

Table of Contents $\quad$ iv

List of Tables $\quad$ x

List of Figures $\quad$ xiv

1 Introduction $\quad 1$

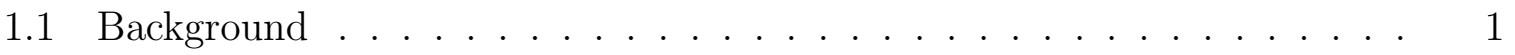

1.2 Previous Literature . . . . . . . . . . . . . . . . 3

1.3 Main Results \& Outline . . . . . . . . . . . . . . . 5

$\begin{array}{lll}2 & \text { The Market Making Problem } & 7\end{array}$

2.1 Formal Description of the Problem . . . . . . . . . . . . 8

2.2 The Solution . . . . . . . . . . . . . . . . . . 12

2.3 Features of the Optimal Strategy . . . . . . . . . . . . . . . . 14

2.4 The Inclusion of Market Orders . . . . . . . . . . . . . . . . . . . . 17

3 Market Making in a Single Asset $\quad 22$

3.1 Introduction . . . . . . . . . . . . . . . . . . 22 
3.2 Arrival of Market Orders and Price Dynamics . . . . . . . . . . . . 25

3.2 .1 Price Dynamics . . . . . . . . . . . . . . . . . . . 25

3.2.2 Self-Exciting Incoming Market Order Dynamics . . . . . . . . 26

3.3 Limit Quote Arrival Dynamics and Fill Rates . . . . . . . . . . . . 29

3.4 Short Term Alpha Dynamics: Directional Strategies and Adverse Selection 33

3.5 The HFT's Optimization Problem . . . . . . . . . . . . . . . . . 34

3.5.1 Formulation of the HF Trading Problem . . . . . . . . . 35

3.5.2 The Feedback Control of the Optimal Trading Strategy . . . . . 37

3.5.3 The Asymptotic Solution of the Optimal Trading Strategy . . . . 39

3.6 HF Market Making, Short Term Alpha, and Directional Strategies . . . . 43

3.7 Conclusions . . . . . . . . . . . . . . . . . . . . . . . 54

4 Algorithmic Trading in Multiple Assets 56

4.1 Introduction $\ldots \ldots \ldots \ldots \ldots \ldots \ldots \ldots$

4.2 The Model . . . . . . . . . . . . . . . . . . . . . . . . . . . 58

4.3 The HFT's Optimization Problem . . . . . . . . . . . . . . . 62

4.3.1 Statement of the Problem . . . . . . . . . . . . . 62

4.3 .2 Solving the Value Function . . . . . . . . . . . . . 64

4.3.3 Analysis of the Optimal Trading Strategy . . . . . . . . . 66

4.4 Simulation Study: PnL of the Strategy . . . . . . . . . . . . . . 73

4.4 .1 Accounting for Adverse Selection $\ldots \ldots \ldots \ldots$

4.4.2 Results of the Simulation Study . . . . . . . . . . . 76 
4.4.3 Comparison to Other Strategies . . . . . . . . . . . . 80

4.5 Numerical Scheme for Exponential Fill Rate . . . . . . . . . . . . . . . 82

4.5.1 Dimensional Reduction of Spatial Variables . . . . . . . . . 82

4.5.2 Description of Numerical Scheme . . . . . . . . . . . 83

4.5.3 Convergence of the Numerical Scheme . . . . . . . . . . 85

4.6 Conclusions . . . . . . . . . . . . . . . . . . . 88

5 The Generalized Latent Hawkes Process $\quad 90$

5.1 Motivation . . . . . . . . . . . . . . . . . 91

5.2 The Generalized Hawkes Process . . . . . . . . . . . . . . . 93

5.2.1 The Model ... . . . . . . . . . . . . . . 93

5.2 .2 The Data . . . . . . . . . . . . . . . . . 94

5.2.3 Probabilistic Quantities of Interest . . . . . . . . . . . . 95

5.2 .4 Renewal Points .................... 96

5.3 Calibration Algorithm I: Isolated Likelihood . . . . . . . . . . . . . 97

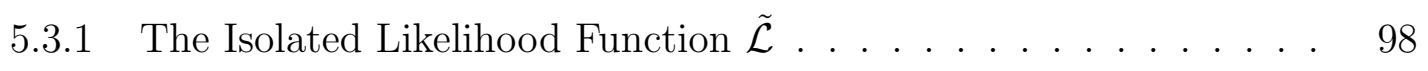

5.3.2 The Calibration Algorithm . . . . . . . . . . . . . . . 99

5.3.3 Bounding the Error of the Isolated Likelihood Function . . . . . . 101

5.3.4 Link to Composite Likelihood Methods . . . . . . . . . . . . . . 101

5.3.5 Numerical Results on Simulated Data . . . . . . . . . . . . . 102

5.4 Calibration Algorithm II: Sequential Monte Carlo . . . . . . . . . . . . . 110

5.4.1 Sequential Monte Carlo \& Particle Filtering - Classical Method . 110 
5.4 .2 The Calibration Algorithm . . . . . . . . . . . . . . . . . 111

5.4.3 Numerical Results on Simulated Data: $\boldsymbol{\eta}(0)=\mathbf{0} \ldots \ldots \ldots \ldots$

5.4.4 Numerical Results on Simulated Data: $\boldsymbol{\eta}(0) \geq 0 \ldots \ldots 117$

$5.5 \overline{\mathcal{F}}_{t}$ - Adapted Estimators for $\boldsymbol{\lambda}_{t} \ldots \ldots \ldots \ldots \ldots \ldots \ldots \ldots$

5.5.1 Via Renewal Points . . . . . . . . . . . . . . . . . . . . . . 122

5.5.2 Via Sequential Monte Carlo . . . . . . . . . . . . . . . . . 122

5.6 Other Applications . . . . . . . . . . . . . . . . . . . . . 125

6 Application to Real Data $\quad 127$

6.1 Statistical Results: Generalized Latent Hawkes Process when $\boldsymbol{\eta}(0)=\mathbf{0}$. 127

6.1.1 Parameter Estimates . . . . . . . . . . . . . . . . 127

6.1.2 Model Validation: Probability Integral Transform . . . . . . . . 131

6.1.3 Model Validation: Probability-Probability Plots . . . . . . . . 137

6.2 Statistical Results: Generalized Latent Hawkes Process when $\boldsymbol{\eta}(0) \geq \mathbf{0}$. 139

6.3 Algorithmic Trading in a Single Asset . . . . . . . . . . . . . . . . 143

6.3.1 The Inclusion of Market Orders . . . . . . . . . . . . . . 145

6.3.2 Performace of the Strategy . . . . . . . . . . . . . . 146

$\begin{array}{llr}7 & \text { Final Conclusions } & 151\end{array}$

7.1 Summary of Contributions . . . . . . . . . . . . . . . . . . 151

7.2 Future Directions . . . . . . . . . . . . . . . . . . . . . 152

$\begin{array}{ll}\text { A Acronyms and Notation } & 155\end{array}$

A.1 Acronyms . . . . . . . . . . . . . . . . . . 155 
A.2 Notation for Chapter $3 \ldots \ldots \ldots \ldots$. . . . . . . . . . . 157

A.3 Notation for Chapter $4 \ldots \ldots \ldots$

A.4 Notation for Chapter $5 \ldots \ldots \ldots$

$\begin{array}{ll}\text { B Supplementary Background Material } & 165\end{array}$

B.1 Hamilton-Jacobi-Bellman Equations . . . . . . . . . . . . . . . . . 165

B.2 Viscosity Solutions . . . . . . . . . . . . . . . . . 167

B.3 Feynman-Kac Forumla for Jump Diffusions . . . . . . . . . . . . 167

$\begin{array}{lr}\text { C Proofs } & 169\end{array}$

C.1 Proof of Lemma $3.2 .2 \ldots \ldots \ldots \ldots$

C.2 Proof of Proposition $3.5 .1 \ldots \ldots \ldots \ldots$

C.3 Proof of Theorem $3.5 .2 \ldots \ldots \ldots \ldots \ldots$

C.4 Proof of Corollary $3.5 .3 \ldots \ldots \ldots \ldots \ldots$

C.5 Proof of Lemma 4.5.1: . . . . . . . . . . . . . . . . . . . 172

C.6 Proof of Lemma 4.5.3: . . . . . . . . . . . . . . . . . . . . . . 173

C.7 Proof of Proposition 4.5.4 (Monotonicity): . . . . . . . . . . . . 174

C.8 Proof of Proposition 4.5.6 (Consistency): . . . . . . . . . . . . . 175

C.9 Proof of Theorem $5.3 .1 \ldots \ldots \ldots \ldots \ldots$

C.10 Proof of Theorem $5.3 .2 \ldots \ldots \ldots \ldots \ldots$

$\begin{array}{lr}\text { D Some Explicit Formulae } & 178\end{array}$

D.1 Exact Optimal Trading Strategy in the Single Asset Hawkes Framework . 178

D.2 Explicit Computation of $b_{\varepsilon} \ldots \ldots \ldots \ldots \ldots \ldots \ldots \ldots$ 
D.3 Conditional Mean of LOB Depth Process . . . . . . . . . . . . . . . 180

$\begin{array}{lr}\text { E Numerical Procedures } & 181\end{array}$

E.1 Fitting the Classical Hawkes Model to Level I Trade Data . . . . . . . . 181

E.2 Simulation Procedure for Section 3.6 . . . . . . . . . . . . . . . . . 182

E.3 Evaluating Non-Trivial Terms in the Numerical Scheme of Section 4.5.2 . 184

E.3.1 The Mixed Partial Term . . . . . . . . . . . . . . . . . . 184

E.3.2 Dimensional Reduction . . . . . . . . . . . . . . . . . . 184

E.3.3 The Neumann Condition . . . . . . . . . . . . . . . . . . . . . . 185

E.3.4 Explicit Example when $n=3 \ldots \ldots \ldots$. . . . . . . 185

E.4 Computing the Posterior Distribution of $\boldsymbol{\lambda}_{t} \ldots \ldots \ldots \ldots$

$\begin{array}{ll}\text { Bibliography } & 187\end{array}$ 


\section{List of Tables}

2.1 Model parameters used in the simulation study for the market making problem. . . . . . . . . . . . . . . . . 16

3.1 The mean and standard deviation of the $\mathrm{PnL}$ from the various strategies as the inventory-management parameter $\phi$ increases, $\varepsilon=0.04$, and final inventory liquidation costs are $1 \mathrm{bps}$ and $10 \mathrm{bps}$ per share. Recall that only the benchmark HFT, who uses $\rho=0.7$, is able to correctly specify the dynamics of short-term-alpha. $\ldots \ldots \ldots \ldots \ldots$

3.2 The mean and standard deviation of the $\mathrm{PnL}$ from the various strategies as the inventory-management parameter $\phi$ increases, $\varepsilon=0.02$, and final inventory liquidation costs are $1 \mathrm{bps}$ and $10 \mathrm{bps}$ per share. Recall that only the benchmark HFT, who uses $\rho=0.7$, is able to correctly specify the dynamics of short-term-alpha. . . . . . . . . . . . . . . 51

3.3 The mean and standard deviation of the $\mathrm{PnL}$ from the various strategies as the inventory-management parameter $\phi$ increases, $\varepsilon=0.04$ and 0.02, and final inventory liquidation costs are $1 \mathrm{bps}$ per share. Recall that only the benchmark HFT, who uses $\rho=0.3$, is able to correctly specify the dynamics of short-term-alpha. $\ldots \ldots \ldots \ldots \ldots$

4.1 Model parameters used in the analysis of the function $g(t, \mathbf{q}, \mathbf{s})$, the continuation region, and the optimal inventory level. Recall that the fill rate assumption $h_{i}^{ \pm}(\delta)=\lambda_{i}^{ \pm} e^{-\kappa_{i}^{ \pm}\left(\delta-\xi_{i}\right)}$ from Example 4.3.3 is being made. . . .

4.2 Model parameters used in the simulation study. Recall that the fill rate assumption $h_{i}^{ \pm}(\delta)=\lambda_{i}^{ \pm} e^{-\kappa_{i}^{ \pm}\left(\delta-\xi_{i}\right)}$ from Example 4.3.3 is being made. . .

5.1 Parameter estimates used for constructing the plots in Figure 5.1. . . . . 
5.2 Mean and standard deviations of estimators (1,000 simulations) when $n=$ $p=2, \boldsymbol{\eta}(0)=\mathbf{0}, \eta_{11}(1)=\eta_{22}(1), \eta_{12}(1)=\eta_{21}(1), \beta_{1}=\beta_{2}, \theta_{1}=\theta_{2}$, and using Algorithm I. Total number of free parameters is 5. LRM refers to the long run mean of activity rate of any component of $\mathbf{N}_{t}$ and runtime is in seconds. . . . . . . . . . . . . . . .

5.3 Mean and standard deviations of estimators (1,000 simulations) when $n=p=2$ and $\boldsymbol{\eta}(0)=\mathbf{0}$ and using Algorithm I. Total number of free parameters is 9

5.4 Quantities associated with judging fixed point stability. 1,000 simulations of 2,000 points when $n=p=2, \boldsymbol{\eta}(0)=\mathbf{0}, \eta_{11}(1)=\eta_{22}(1), \eta_{12}(1)=\eta_{21}(1)$, $\beta_{1}=\beta_{2}, \theta_{1}=\theta_{2}$, and using Algorithm I. . . . . . . . . 108

5.5 Quantities associated with judging fixed point stability. 1,000 simulations of 10,000 points when $n=p=2, \boldsymbol{\eta}(0)=\mathbf{0}, \eta_{11}(1)=\eta_{22}(1), \eta_{12}(1)=$ $\eta_{21}(1), \beta_{1}=\beta_{2}, \theta_{1}=\theta_{2}$, and using Algorithm I.

5.6 Quantities associated with judging fixed point stability. 1,000 simulations of 2,000 points when $n=p=2, \boldsymbol{\eta}(0)=\mathbf{0}$, and using Algorithm I. This is the non-symmetric case.

5.7 Quantities associated with judging fixed point stability. 1,000 simulations of 10,000 points when $n=p=2, \boldsymbol{\eta}(0)=\mathbf{0}$, and using Algorithm I. This is the non-symmetric case. . . . . . . . . . . . . . . . .

5.8 Mean and standard deviations of estimators (1,000 simulations) when $n=$ $p=2, \boldsymbol{\eta}(0)=\mathbf{0}, \eta_{11}(1)=\eta_{22}(1), \eta_{12}(1)=\eta_{21}(1), \beta_{1}=\beta_{2}, \theta_{1}=\theta_{2}$, and using Algorithm II. Total number of free parameters is 5 . . . . . . . . .

5.9 Mean and standard deviations of estimators (1,000 simulations) when $n=$ $p=2, \boldsymbol{\eta}(0)=\mathbf{0}$, and using Algorithm II. Total number of free parameters is 9 .

5.10 Mean and standard deviations of estimators (250 simulations) when $n=$ $p=2, \eta_{11}(1)=\eta_{22}(1), \eta_{12}(1)=\eta_{21}(1), \beta_{1}=\beta_{2}, \theta_{1}=\theta_{2}$, and using Algorithm II. Total number of free parameters is $7 . \ldots \ldots \ldots$

5.11 Mean and standard deviations of estimators (250 simulations) when $n=$ $p=2$ and using Algorithm II. Total number of free parameters is 13. 
6.1 Mean and standard deviations of estimators on historical data when $n=$ $p=2, \boldsymbol{\eta}(0)=\mathbf{0}, \eta_{11}(1)=\eta_{22}(1), \eta_{12}(1)=\eta_{21}(1), \beta_{1}=\beta_{2}, \theta_{1}=\theta_{2}$, and using Algorithm I with a cluster size of $m=4$. Total number of free parameters is 5. LRM refers to the long run mean of activity rate of any component of $\mathbf{N}_{t}$, given by Lemma 3.2.2. Sample consists of disjoint 60-minute windows between 10:00am and 4:00pm from Jan-May 2011. . .

6.2 Mean and standard deviations of estimators on historical data when $n=$ $p=2, \boldsymbol{\eta}(0)=\mathbf{0}$, and using Algorithm I with a cluster size of $m=4$. $L R M$ refers to the long run mean of activity rate of $\mathbf{N}_{t}$, given by Lemma 3.2.2. Sample consists of disjoint 60-minute windows between 10:00am and 4:00pm from Jan-May 2011. . . . . . . . . . . . . . .

6.3 Sample correlation of selected pairs of estimators when the calibration does not impose symmetry. . . . . . . . . . . . . . . . . . .

6.4 Parameter estimates used for PIT and P-P model diagnotics for the 2:00$3: 00$ window of May 31, 2011. . . . . . . . . . . . . . . 131

6.5 Parameter estimates used for PIT and P-P model diagnotics for the 3:004:00 window of May 31, 2011. . . . . . . . . . . . . .

6.6 Mean and standard deviations of estimators on historical data when $n=$ $p=2, \eta_{11}(1)=\eta_{22}(1), \eta_{12}(1)=\eta_{21}(1), \beta_{1}=\beta_{2}, \theta_{1}=\theta_{2}$, and using Algorithm II with 200 particles. Total number of free parameters is 7 . $L R M$ refers to the long run mean of activity rate of any component of $\mathbf{N}_{t}$. Sample consists of disjoint 60-minute windows between 10:00am and 4:00pm from May 2011. . . . . . . . . . . . . . . .

6.7 Mean and standard deviations of estimators on historical data when $n=$ $p=2$ and using Algorithm II with 200 particles. Total number of free parameters is 13. Sample consists of disjoint 60-minute windows between 10:00am and 4:00pm from May 2011. . . . . . . . . . . .

6.8 Sample correlation of seletced pairs of estimators when the calibration does not impose symmetry for general $\boldsymbol{\eta}(0) \geq \mathbf{0} \ldots \ldots \ldots \ldots$

6.9 Fitted parameters to be used in the backtesting of our single asset market making strategy. Sample period is Jan-Apr 2011. . . . . . . . . . . 144

6.10 Other model parameters to be used in the backtesting of the single asset market making strategy. . . . . . . . . . . . . . . 144 
6.11 Daily PnL results for BBBY. The asset's daily closing price ranged from approximately $\$ 45$ to $\$ 58$ throughout the trading period. . . . . . . . . 147

6.12 Daily PnL results for MSFT. The asset's daily closing price ranged from approximately $\$ 25$ to $\$ 29$ throughout the trading period. . . . . . . . . 148

6.13 Daily PnL results for TEVA. The asset's daily closing price ranged from approximately $\$ 45$ to $\$ 57$ throughout the trading period. . . . . . . . . . 149 


\section{List of Figures}

2.1 A snapshot of the (NASDAQ) LOB for IBM on June 21, 2011 at 11:45:20.26. 8

2.2 Fill rates $\Lambda^{ \pm}$with $\kappa_{t}^{+} \equiv 2, \kappa_{t}^{-} \equiv 1, \lambda_{t}^{+} \equiv 0.75$ and $\lambda_{t}^{-} \equiv 1$. As $\kappa_{t}^{ \pm}$ evolve, the fill rate shape changes, while the market order activity rates $\lambda^{ \pm}$modulate the vertical scale. The maximum fill rate is achieved at $\delta=0.10$

2.3 Optimal posting depth of limit buy quotes $\delta_{t}^{-}$for various inventory levels $q$ versus time with terminal investment horizon $T=120$ and maximum inventory size, for the purpose of numerically solving (2.10), of 40 units

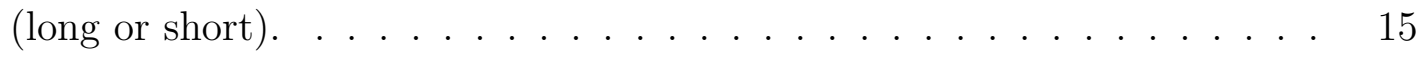

2.4 Distributional properties associated with the optimal strategy for various inventory-aversion levels,$\phi \ldots \ldots \ldots \ldots$

2.5 Performance of strategy for various $\phi$, ranging from 0.3 to 200 , with and without market orders. . . . . . . . . . . . . . . . . . 19

2.6 Distribution of the number of executed marker orders for various $\phi$. . . . 20

2.7 Distribution of $\max _{0 \leq t \leq T}\left\{\left|q_{t}\right|\right\}$ for various $\phi \ldots \ldots \ldots \ldots$

3.1 IBM market orders. market orders. Historical running intensity versus smoothed fitted intensity using a 1 second sliding window for IBM for a 2 minute period, between 3:30pm and 3:32pm, February 1, 2008. . . . .

3.2 Sample path of market order activity rates. When influential trades arrive, the activity of both buy and sell orders increase but by differing amounts. Circles indicate the arrival of an influential market order, while squares indicate the arrival of non-influential trades. . . . . . . . . . . 
3.3 Mean inventory drift. . . . . . . . . . . . . . . . . .

3.4 A sample path of the HFTs' interpretation of the stochastic drift, MO activity, and FP parameter. The benchmark model has the correct data generating path, while the others under- or over-estimate the role of trade

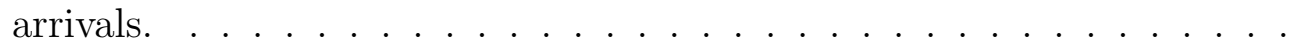

3.5 A sample path generated by the strategy under an exponential fill rate. When influential trades arrive, the activity of both buy and sell orders increase but by differing amounts, the FP parameters also jump, and both decay to their long-run levels. Circles indicate the arrival of an influential market order, while squares indicate the arrival of non-influential trades. Open symbols indicate those MOs which do not fill the trader's LO, while filled in symbols indicate those MOs which do fill the trader's LO. . . . .

$4.1 \mathrm{~g}$ surface for various $\mathbf{s}$. Figures 4.1(a) and 4.1(c) show the surface and heatmap, respectively, of $g(t, \mathbf{s}, \mathbf{q})$ when the assets' midprices are equal. Figures 4.1(b) and 4.1(d) show the surface and heatmap, respectively, of $g(t, \mathbf{s}, \mathbf{q})$ when Asset 1 is cheap relative to Asset $2\left(s_{1}=s_{2}-0.012\right) \ldots$.

4.2 Continuation region for various $\mathbf{s}$. The different coloured regions correspond to different MO submissions. Namely, light blue: Asset 1 buy MO; orange: Asset 1 sell MO; dark blue: Asset 2 buy MO; burgundy: Asset 2 sell MO; green: continuation region; and white: unattainable. Recall that Figure $4.2(\mathrm{~b})$ is specified by $s_{1}=s_{2}-0.012 \ldots \ldots \ldots \ldots$

4.3 Inventory drift when the investor is flat (i.e., $q_{1}=q_{2}=0$ ). . . . 70

4.4 Inventory drift when the assets' midprices are equal (i.e., $s_{1}=s_{2}$ ). . . 71

4.5 Inventory drift when Asset 1 is cheap relative to Asset 2 (i.e., $s_{1}=s_{2}-0.012$ ). 71

4.6 Mean reversion point of $\mathbf{q}$ as a function of $s_{1}-s_{2}$. The red (blue) dots correspond to small (large) values of $s_{1}-s_{2} \ldots \ldots \ldots \ldots \ldots$

4.7 Sample paths of the midprice process $\mathbf{S}_{t}$ and drift process $\boldsymbol{\alpha}_{t}$. The red lines in Figure 4.7(c) represent the boundary of the no-arbitrage region. . 76

4.8 Sample paths of the investor's LO depth and her inventory level for $\phi=10.77$ 
4.9 Distribution of terminal profit and loss. The probability of an informed MO is fixed at $\rho=0.50 \ldots \ldots \ldots \ldots \ldots \ldots \ldots$

4.10 Performance of strategy for various $\phi$ with different drift impact scale parameters $\epsilon$. The probability of an informed MO is fixed at $\rho=0.50$. .

4.11 Performance of strategy for various $\phi$ with different probabilities of informed MOs $\rho$. The drift impact scale parameter is fixed at $\epsilon=0.01$. .

4.12 Distribution of the number of executed MOs for various inventory penalty levels when $\epsilon=0.01$ and $\rho=0.50 \ldots \ldots \ldots \ldots$

4.13 Comparison of efficient frontiers for the full strategy (solid lines), the no boundary strategy (dashed line), and the single asset benchmark (dotted line). The blue curves refer to $\epsilon=0$ and the red curves refer to $\epsilon=0.02$. In both cases, $\rho=0.50 \ldots \ldots \ldots \ldots \ldots \ldots$

5.1 IBM market orders. Historical running intensity versus smoothed fitted intensity using a 1 second sliding window for IBM for a 2 minute period, between 3:30pm and 3:32pm, February 1, 2008. . . . . . . . . .

5.2 A graphical model representation of dependencies in our self-exciting process with random jump sizes $\boldsymbol{\eta}_{t_{k}} \ldots \ldots \ldots \ldots \ldots \ldots$

5.3 Distribution of estimators (1,000 simulations of 2,000 data points) when $n=p=2, \boldsymbol{\eta}(0)=\mathbf{0}, \eta_{11}(1)=\eta_{22}(1), \eta_{12}(1)=\eta_{21}(1), \beta_{1}=\beta_{2}, \theta_{1}=\theta_{2}$, and using Algorithm I with $m=3 . \ldots \ldots \ldots \ldots$

5.4 Distribution of estimators (1,000 simulations of 2,000 data points) when $n=p=2, \boldsymbol{\eta}(0)=\mathbf{0}$, and using Algorithm I with $m=3$. This is the non-symmetric case.

5.5 Distribution for the number of iterations of the calibration algorithm on 1,000 simulations of 2,000 points in the symmetric model. . . . . . . . . 109

5.6 Distribution of estimators (1,000 simulations of 2,000 data points) when $n=p=2, \boldsymbol{\eta}(0)=\mathbf{0}, \eta_{11}(1)=\eta_{22}(1), \eta_{12}(1)=\eta_{21}(1), \beta_{1}=\beta_{2}, \theta_{1}=\theta_{2}$, and using Algorithm II with 20 particles. . . . . . . . . . . 115 
5.7 Distribution of estimators (1,000 simulations of 2,000 data points) when $n=p=2, \boldsymbol{\eta}(0)=\mathbf{0}$, and using Algorithm II with 20 particles. This is the non-symmetric case.

5.8 Distribution of estimators (250 simulations of 2,000 data points) when $n=p=2$ and using Algorithm II with 50 particles. This is the non-

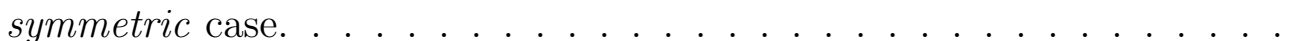

5.9 Distribution of estimators (250 simulations of 2,000 data points) when $n=p=2$ and using Algorithm II with 50 particles. This is the nonsymmetric case. . . . . . . . . . . . . . . . .

5.10 Propagation of the progressively measureable particle filter using the mean of 3 particles via SMC with simulated data. . . . . . . . . . . . . . .

6.1 Frequency of parameter estimates when constrained to $n=p=2, \boldsymbol{\eta}(0)=$ $\mathbf{0}, \eta_{11}(1)=\eta_{22}(1), \eta_{12}(1)=\eta_{21}(1), \beta_{1}=\beta_{2}, \theta_{1}=\theta_{2}$, and using Algorithm I. Sample consists of disjoint 60-minute windows between 10:00am and 4:00pm from Jan-May 2011.

6.2 A workflow diagram representing the PIT calculation of the $i^{\text {th }}$ particle for the $k^{\text {th }}$ interarrival time $T_{k} \ldots \ldots \ldots \ldots$

6.3 Distribution of the PIT quantities when constrained to $n=p=2, \boldsymbol{\eta}(0)=$ $\mathbf{0}, \eta_{11}(1)=\eta_{22}(1), \eta_{12}(1)=\eta_{21}(1), \beta_{1}=\beta_{2}, \theta_{1}=\theta_{2}$, and using Algorithm I for the 2:00-3:00 window.

6.4 Distribution of the PIT quantities when constrained to $n=p=2, \boldsymbol{\eta}(0)=$ $\mathbf{0}, \eta_{11}(1)=\eta_{22}(1), \eta_{12}(1)=\eta_{21}(1), \beta_{1}=\beta_{2}, \theta_{1}=\theta_{2}$, and using Algorithm I for the 3:00-4:00 window.

6.5 Autocorrelation function of the PIT quantities when constrained to $n=$ $p=2, \boldsymbol{\eta}(0)=\mathbf{0}, \eta_{11}(1)=\eta_{22}(1), \eta_{12}(1)=\eta_{21}(1), \beta_{1}=\beta_{2}, \theta_{1}=\theta_{2}$, and using Algorithm I for the 2:00-3:00 window. The blue lines indicate 95\% confidence intervals. . . . . . . . . . . . . . . . . . .

6.6 Autocorrelation function of the PIT quantities when constrained to $n=$ $p=2, \boldsymbol{\eta}(0)=\mathbf{0}, \eta_{11}(1)=\eta_{22}(1), \eta_{12}(1)=\eta_{21}(1), \beta_{1}=\beta_{2}, \theta_{1}=\theta_{2}$, and using Algorithm I for the 3:00-4:00 window. The blue lines indicate 95\% confidence intervals. . . . . . . . . . . . . . . . . . . 136 
6.7 Exponential P-P plots when constrained to $n=p=2, \boldsymbol{\eta}(0)=\mathbf{0}, \eta_{11}(1)=$ $\eta_{22}(1), \eta_{12}(1)=\eta_{21}(1), \beta_{1}=\beta_{2}, \theta_{1}=\theta_{2}$, and using Algorithm I. . . . 138

6.8 Frequency of parameter estimates when constrained to $n=p=2, \eta_{11}=$ $\eta_{22}, \eta_{12}=\eta_{21}, \beta_{1}=\beta_{2}, \theta_{1}=\theta_{2}$, and using Algorithm II. . . . . . . . 142

6.9 Distribution of $\max _{0 \leq t \leq T}\left\{\left|q_{t}\right|\right\}$ for various MO aggression levels, $\bar{\alpha}$. The inventory aversion level is fixed at $\phi=10^{-7}$. There are 1,386 5-minute trading intervals in this sample. . . . . . . . . . . . . . 150 


\section{Chapter 1}

\section{Introduction}

\section{$1.1 \quad$ Background}

Most of the traditional stock exchanges have converted from open outcry communications between human traders to electronic markets where the activity between participants is handled by computers. In addition to those who have made the conversion, such as the New York Stock Exchange (NYSE) and the London Stock Exchange, new electronic trading platforms have entered the market - for example, NASDAQ in the US and Chi-X in Europe. Along with the exchanges, market participants have been increasingly relying on the use of computers to handle their trading needs. Initially, computers were employed to execute trades, but today computers manage inventories and make trading decisions; this modern way of trading in electronic markets is known as algorithmic trading (AT).

In the past, markets were quote driven which means that market makers (MMs) quoted buy and sell prices and investors would trade with them. Today, there are limit order markets where all participants can post limit buy or sell orders, i.e., behave as market makers in the old quote driven market. The limit orders show an intention to buy or sell and must indicate the number of shares and the price at which the agent is willing to trade. The limit buy (sell) order with the highest (lowest) price tag is known as the best bid (best offer). During the trading day, all orders are accumulated in the limit order book (LOB) until they find a counterparty for execution or are cancelled by the agent who posted them. The counterparty is a market order which is an order to buy or sell a number of shares, regardless of the price, which is immediately executed against limit orders resting in the LOB at the best execution prices.

Trade initiation may be motivated by many factors which have been extensively stud- 
ied in literature; see, for example, Sarkar and Schwartz (2009). Some of these include asymmetric information, differences in opinion or differential information, and increased proportion of impatient (relative to patient) traders. Likewise, trade clustering can be the result of various market events; see Cartea and Jaimungal (2013a). For instance, increases in market activity could be due to shocks to the fundamental value of the asset, or the release of public or private information that generates an increase in trading (twosided or one-sided) until all information is impounded in stock prices. However, judging by the sharp rise of AT in the recent years and the explosion in the volume of submissions and order cancellations, it is also plausible to expect that certain AT strategies that generate trade clustering are not necessarily motivated by the factors mentioned above. An extreme example occurred during the flash crash of May 6, 2010 where it is clear that trading between high frequency traders (HFTs) generated more trading giving rise to the "hot potato" effect. ${ }^{1}$

The profitability of these low latency AT strategies depends on how they interact with the dynamics of the LOB and, more importantly, how these AT strategies coexist. The recent increase in the number of orders and in the frequency of LOB updates shows that fast traders are responsible for most of the market activity, and it is very difficult to link news arrival or other classical ways of explaining motives for trade to the activity one observes in electronic markets. Superfast algorithms make trading decisions in split milliseconds. This speed, and how other superfast traders react, makes it difficult to link trade initiation to private or public information arrival, a particular type of trader, liquidity shock, or any other market event.

Very little is known about the details of the strategies that are employed by AT desks or the more specialized proprietary high frequency trading trading (HFT) desks. Algorithms are designed for different purposes and to seek profits in different ways (see Bouchard et al. (2011)). For example, there are algorithms that are designed to find the best execution prices for investors who wish to minimize the price impact of large buy or sell orders (see Almgren and Chriss (2000), Kharroubi and Pham (2010), and Bayraktar and Ludkovski (2012)), while others are designed to manage inventory risk; see Guéant et al. (2013). There are high frequency (HF) strategies that specialize in arbitraging across different trading venues, while others seek to profit from short-term deviations in stock prices. And finally, there are trading algorithms that seek to profit from providing liquidity by posting bids and offers simultaneously; see Guilbaud and Pham (2013b) and Cartea et al. (2013). In previous works on algorithmic trading in LOBs, the mid-price is assumed to be independent of the arrival and volume of market orders, and market orders arrive at Poisson times. The works presented in Chapter 3 and Chapter 4 differ

\footnotetext{
${ }^{1}$ During that day between 13:45:13 and 13:45:27 CT, HFTs traded over 27,000 contracts which accounted for approximately $49 \%$ of the total trading volume, while their net position changed by only about 200 contracts (see Kirilenko et al. (2010)).
} 
significantly in that we do not assume independence between the arrival of market orders, the LOB, and mid-price processes.

The increase in computing power has made it easier for market participants to deploy ever more complicated trading strategies to profit from changes in market conditions. Key to the success of HF strategies is the speed at which agents can process information and news events to take trading decisions. A unique characteristic of HF trading is that the strategies are designed to hold close to no inventories over very short periods of time (seconds, minutes, or at most one day) to avoid both exposure to markets after close and the need to post collateral overnight. Thus, profits are made by turning over positions very quickly to make a very small margin per roundtrip transaction (buy followed by a sell or vice-versa) and repeating it as many times as possible during each trading day.

Despite the substantial changes that markets have undergone in the recent past (see Cvitanic and Kirilenko (2010)), some strategies used by investors remain the same. When asked about how to make money in the stock market, an old adage responds "Buy low and sell high." Although in principle this sounds like a good strategy, its success relies on spotting opportunities to buy and sell at the right time. Surprisingly, more than ever, due to the incredible growth in computing power, a great deal of the activity in the US and European stock exchanges is based on trying to profit from short-term price predictions by buying low and selling high. ${ }^{2}$ The effectiveness of these computerized short-term strategies, a subset of AT known as HFT, depends on the ability to process information and send messages to the electronic markets in microseconds; see Cartea and Penalva (2012). In this thesis, we develop HF trading strategies in a variety of market settings that profit from having access to a low latency connection with the exchange and to decide when and how to enter and exit the market over extremely short time intervals.

\subsection{Previous Literature}

In light of the technological advances highlighted in Section 1.1, there has also been a flurry of activity in academic research which has addressed a variety of interesting problems. ${ }^{3}$ Large-scale macroscopic modeling assumptions in classical mathematical finance literature (see, e.g., Kou (2002) for the modeling of asset prices as jump diffusions, Heston (1993) for option pricing with stochastic volatility, and Carr et al. (2003) for stochastic volatility with Lévy models) give rise to overly simplistic and unrealistic microstructure dynamics. Due to the high speed nature of these trading algorithms, it has become a

\footnotetext{
${ }^{2}$ See, for example, CFTC and SEC (2010) and The Government Office for Science, London (2012).

${ }^{3}$ See Hasbrouck and Saar (2013) for a study on the effects that low-latency trading has on short-term volatility, bid/offer spread, and depth of the LOB.
} 
neccessity to model tick data directly with careful considerations given to the bid/offer spread, liquidity (or lack thereof), market impact of trading, and the overall structure of the LOB.

In response to such shortcomings, there have been several attempts to model this event data directly. Cont et al. (2010) pose a discrete-space endogenous queuing model ${ }^{4}$ for the limit order book that directly models limit order (LO) submissions/cancellations and market order (MO) arrivals. Roşu (2009) develops a stylistic model that includes different types of agents (i.e., aggressive vs passive, patient vs impatient, etc). Finally, Shek (2011) uses trade size and LOB imbalance in conjunction with a marked bivariate Hawkes process to model MO arrivals. See Madhavan (2000) for a detailed survey on market microstructure modeling. Advancements on the front of event modeling in tick data via Hakwes processes are further discussed in Chapter 5.

Although some of the previously mentioned authors have touched on the performance of particular trading strategies in their respective modeling frameworks, special attention should be given to papers that address HFT problems directly in a more systematic framework. One of the first problems to get the attention of academics is that of optimal liquidation/acquisition where a firm must liquidate (or acquire) a fixed, large number of units of an asset by some fixed time horizon. Classical quantitative finance assumes frictionless markets and the absence of any price impact as a result of trading - assumptions that are clearly violated in practice when attempting to acquire a large sum of shares in a short period of time.

To this end, the pivotal research done in Almgren and Chriss (2000) was one of the first to directly address the issue of market impact in the context of optimal execution in a continuous time setting ${ }^{5}$, and is considered by some to be one of the most influential papers within the scope of high frequency optimal execution literature. Several risk metrics were considered in this piece including a modification to VaR (value at risk) that accounts for liquidity in a way that explicitly considers the tradeoff between volatility risk and liquidation costs.

There are countless extensions to the work presented in Almgren and Chriss (2000). Obizhaeva and Wang (2013) considers the combined discrete and continuous trading problem, and Alfonsi and Fruth (2010) allow for a general LOB shape function. Alfonsi et al. (2012) extend the relation between reslience functions and arbitrage to include the weaker notion transaction-triggered price manipulation which results in an agent (who is responsible for a large acquisition) to trade in quantities far larger than the total lot in an attempt to reduce overall transaction costs (in contrast to classical arbitrage which

\footnotetext{
${ }^{4}$ See Horst and Rothe (2008) for another example of an agent-based queuing model.

${ }^{5}$ Bertsimas and Lo (1998) were the first authors to solve such a problem in discrete time.
} 
allows the agent to produce a profit). Modifications to allow the investor to place market orders and take liquidity from the market can be found in Cartea and Jaimungal (2014) where the authors use TWAP ${ }^{6}$ as a benchmark. The inclusion of a price limiter, where the agent is unwilling to pay above a certain price for any number of shares in her acquisition strategy, is done in Jaimungal and Kinzebulatov (2014). Guéant et al. (2013) focus specifically on the dynamics initiated by the agent's aggressive orders hitting the LOB. The accelerated share repurchase problem is addressed in an optimal control framework in Jaimungal et al. (2013). Finally, Almgren (2012) revisits the optimal execution problem in the presence of varying market liquidity and stochastic volatility.

There has also been a rapidly increasing amount of attention given to HFT problems where where traders are allowed to both buy and sell assets, rather than trade in only one direction as in the liquidation problem. That is, if the agent trades using only limit orders, she behaves somewhat like a traditional market maker. This movement was lead by Avellaneda and Stoikov (2008) where the authors apply the theory of stochastic optimal control to a utility maximization problem in the context of high frequency trading. Although the underlying modeling assumptions are simplistic relative to the aforementioned works, this work was unique in the sense that it directly maximized terminal utility as a result of trading (in contrast to minimizing the cost to liquidate a given number of shares). There is no doubt that the results presented Avellaneda and Stoikov (2008) have greatly motivated the original research presented in Chapter 3 and, ultimately, this entire thesis.

\subsection{Main Results \& Outline}

This thesis establishes mathematically rigorous frameworks for market microstructure and constructs optimal HFT strategies within such frameworks, while keeping practical considerations in mind. The main theoretical tools that will be employed are stochastic optimal control theory, sequential monte carlo, and particle filtering methods.

While most of the published works referenced in Section 1.2 are focused on either the theoretical aspects of HFT problems or carry out empirical studies on real data, this thesis is aimed at providing a seamless bridge between these two focuses. More specifically, special considerations are given to real-world applications of derived theoretical concepts. Moreover, an entire chapter is dedicated to providing methods for the application of the

\footnotetext{
${ }^{6}$ A Time Weighted Average Price (TWAP) execution strategy refers to buying shares at a constant rate with respect to time. Similarly, a Volume Weighted Average Price (VWAP; see Guéant and Royer (2014) and Frei and Westray (2013)) execution strategy refers to buying shares at a rate that is proportional to the current volume being traded at that time.
} 
involved Generalized Hawkes model that is presented in a market making problem, and a further chapter is devoted to the application of these results (and the corresponding HFT strategy) to real data. The author has made every effort to ensure that the mathematical results presented in this thesis can be applied in real world scenarios.

This thesis contains the first work to solve a stochastic control problem that explicitly incorporates both adverse selection and a self-exciting point process for modeling event data in the context of HF market making. ${ }^{7}$ Full calibration and estimation methodologies are also provided for the probabilistic model introduced in this piece to ensure a seamless bridge between the theory and application. This thesis is also the first to contain work related to HFT strategies that profit statistical arbitrage opportunities that arise in economies where the midprice process ventures close to the no-arbitrage boundary. The structure present in such economic systems is made mathematically precise and the optimal HFT strategy is derived, analyzed, and tested in a simulation study that includes adverse selection. An explicit numerical scheme, along with a dimensional reduction, is provided for solving this problem and we prove that this scheme converges to the corresponding viscosity solution as the discretized time and space step sizes become small. Considerations regarding numerical techniques for all results presented in this thesis are discussed at length to ensure that all strategies can be implemented in real-time.

This thesis is organized as follows: Chapter 2 introduces the market making problem in a formal mathematical setting with a simplified example and discusses some stylized features of the solution. Chapter 3 poses and solves a stochastic control problem associated with market making in a single asset in the prescence of adverse selection and market order clustering. Chapter 4 poses and solves a stochastic control problem associated with algorithmic trading in multiple assets that are structurally dependent (in some well defined way). Chapter 5 introduces a further generalization to the process discussed in Chapter 3, as well as provides efficient parameter calibration algorithms and real-time estimation of the latent intensity process. Chapter 6 applies some of the previously derived results to real-world data. Finally, Chapter 7 summarizes the conclusions of this thesis and outlines directions for future work in this field. A number of Appendicies (A to E) also provide a notation index, extra background material, proofs, some explicit formulae, and details regarding numerical procedures.

\footnotetext{
${ }^{7}$ This work is common to Cartea et al. (2014).
} 


\section{Chapter 2}

\section{The Market Making Problem}

A pillar of capital markets is the provision of liquidity to investors when they need it. As compensation for providing immediacy, market makers or liquidity providers earn the realized spread, which is the market maker's expected gain from a roundtrip trade (i.e. a buy followed by a sell or vice-versa). These expected gains depend on, among other things, the architecture of the limit order book (LOB), and on the ability that market makers have to hold inventories which gives them the opportunity to build strategic long or short positions.

In the LOB, limit orders are prioritized first according to price and then according to time. ${ }^{8}$ For example, if two sell (buy) limit orders are sent to the exchange, the one with the lowest (highest) price is placed ahead in the queue. Similarly, orders that improve the prices for buy or sell will jump ahead of others regardless of how long they have been resting in the book. Thus, based on the price/time priority rule the LOB stacks on one side all buy orders (also referred to as bids) and on the other side all sell orders (also referred to as offers). The difference between the best offer and best bid is known as the spread, and their mean is referred to as the midquote price. Another dimension of the book is the quantities on the sell and buy sides for each price tick which give "shape" to the LOB. See Figure 2.1 for a visual snapshot of the LOB for IBM.

The role of the market maker (MM) is to provide the market with liquidity without the intention of holding large (either long or short) inventories for an extended period of time. The market making problem is to devise how one would send limit quotes to the LOB in an optimal way (subject to some perfomance criterion). Carmona and Webster

\footnotetext{
${ }^{8}$ This is the case for most exchanges. Some exchanges use prorata order books, where market orders are matched with all traders posting at the best bid or offer proportional their posted volume (see, e.g., Guilbaud and Pham (2013a)) or other alternatives such as Broker priority in Scandinavian markets.
} 


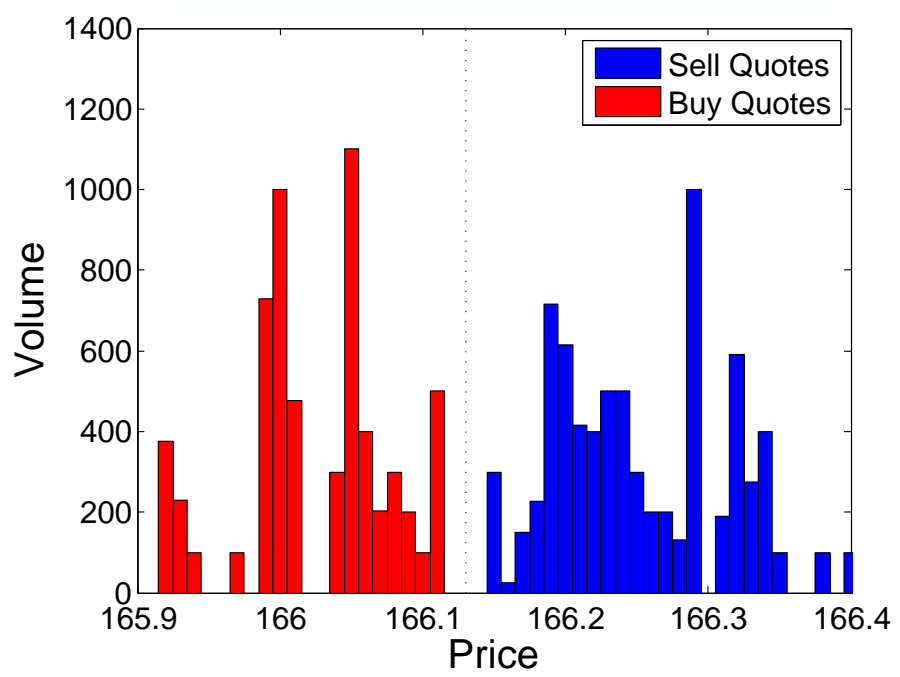

Figure 2.1: A snapshot of the (NASDAQ) LOB for IBM on June 21, 2011 at 11:45:20.26.

(2012b) proposes an equilibrium model showing how such MMs provide liquidity.

In this chapter, two simplified formulations of the market making problem are presented as groundwork for more involved settings (as proposed in Chapters 3 and 4). Section 2.1 gives a formal description of the stochastic control problem. Sections 2.2 and 2.3 derive the solution and describe the financial intuition embedded in the form of the optimal control, respectively. Finally Section 2.4 discusses the effect of allowing the MM to also execute market orders (MOs). The reader is encouraged to consult Appendix B.1 for background information on stochastic control theory and the general form of the corresponding Hamilton-Jacobi-Bellman (HJB) equations.

\subsection{Formal Description of the Problem}

In this section, a high frequency marking making problem similar to that of Avellaneda and Stoikov (2008) is described. More specifically, the MM is to select the optimal depth to post limit quotes on both sides of the LOB with a fixed volume (normalized to one unit of inventory). If we denote the midprice process by $\left\{S_{t}\right\}_{0 \leq t \leq T}$ and the depth of the MM's posted limit buy (resp. sell) quote is denoted $\delta^{-}\left(\operatorname{resp} . \delta^{+}\right)$, then the limit buy (sell) quote would be posted at $S_{t}-\delta^{-}\left(\operatorname{resp} . S_{t}+\delta^{+}\right)$. That is, the strategy $\boldsymbol{\delta}=\left(\delta^{-}, \delta^{+}\right)$ describes where the MM posts her quotes relative to the midprice. 
We assume that the midprice (or fundamental price) of the traded asset follows

$$
d S_{t}=\sigma d W_{t}
$$

where $\left\{W_{t}\right\}_{0 \leq t \leq T}$ is a $\mathbb{P}$-standard Brownian Motion ${ }^{9}$ (for some $T<\infty$ ), and $S_{0}>0$ and $\sigma>0$ are constants. ${ }^{10}$ In this chapter, we do not assume any adverse selection effects (i.e. the drift of $S_{t}$ is always zero). The discussion on adverse selection and its connection to short-term-drift is postponed to Section 3.4.

The only other sources of randomness in this simplified framework are the dynamics describing incoming market orders, the shape of the limit order book, and the distribution governing the size of incoming market orders.

Let $\left\{M_{t}^{-}\right\}_{0 \leq t \leq T}$ and $\left\{M_{t}^{+}\right\}_{0 \leq t \leq T}$ denote the counting processes associated with number of market orders up to time $t$. The sell market orders (that fill limit buy quotes) correspond to $M_{t}^{-}$, and the buy market orders (that fill limit sell quotes) correspond to $M_{t}^{+}$. We assume that $M_{t}^{-}$and $M_{t}^{+}$are independent poisson processes (and also independent of $W_{t}$ ) with constant rates $\lambda^{-}$and $\lambda^{+}$, respectively.

The remaining two sources of randomness (the shape of the limit order book and the distribution governing the size of incoming market orders) can be combined into one assumption. The MM really only cares about the rate, per unit of time, that her limit quote gets filled as a function of the depth $\delta$. We assume that at depth $\delta^{ \pm}$, the MM gets filled at rate $\Lambda^{ \pm}:=\lambda^{ \pm} \exp \left\{-\kappa^{ \pm} \delta^{ \pm}\right\}$when $\delta^{ \pm} \geq 0$ for some positive constants $\lambda^{ \pm}$and $\kappa^{ \pm}{ }^{11}$ Observe the trade off for selecting different depths of $\delta$. When $\delta$ is small, then the arrival rate of filled quotes increases, but the executed price is worse (from the point of view of the MM). Alternatively, larger values of $\delta$ will cause fewer filled limit quotes, but each filled quote will occur a better price.

Figure 2.2 illustrates the shape of the fill rates at time $t$ describing the rate of arrival of market orders which fill limit orders placed at price levels $S_{t} \pm \delta_{t}^{ \pm}$. Notice that these rates peak at zero spread at which point they are equal to the arrival rate of market orders. In the figure, these rates are asymmetric and decay at differing speeds because we have assumed different parameters for the buy and sell sides, $\kappa_{t}^{+} \equiv 2, \kappa_{t}^{-} \equiv 1, \lambda_{t}^{+} \equiv 0.75$ and $\lambda_{t}^{-} \equiv 1$.

\footnotetext{
${ }^{9}$ The results in this chapter still hold if we instead model $S_{t}$ as a compensated jump process, provided that $S_{t}$ meets some integrability conditions.

${ }^{10}$ Unless otherwise stated, all random variables and stochastic processes in this chapter are defined on the completed, filtered probability space $\left(\Omega, \mathcal{F}_{T}, \mathbb{F}, \mathbb{P}\right)$ with filtration $\mathbb{F}=\left\{\mathcal{F}_{t}\right\}_{0 \leq t \leq T}$ and where $\mathbb{P}$ is the real-world probability measure. In this chapter, $\mathbb{F}$ is the natural filtration generated by $\left(W_{t}, N_{t}\right)$, all of which will be defined later.

${ }^{11}$ This assumption can also be derived by assuming that the LOB volume is constant and MO volumes are iid exponential random variables.
} 


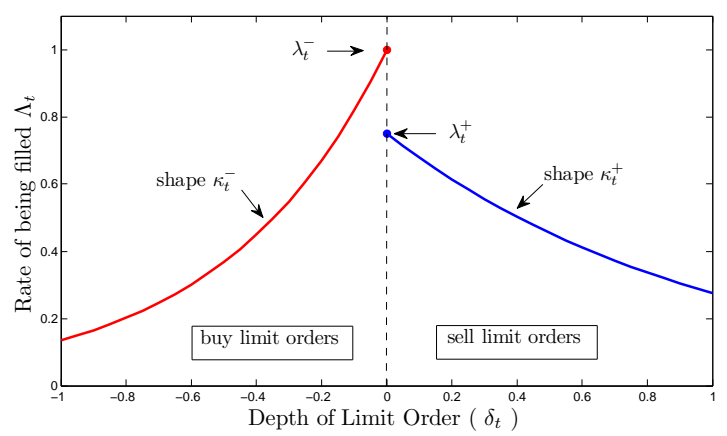

Figure 2.2: Fill rates $\Lambda^{ \pm}$with $\kappa_{t}^{+} \equiv 2, \kappa_{t}^{-} \equiv 1, \lambda_{t}^{+} \equiv 0.75$ and $\lambda_{t}^{-} \equiv 1$. As $\kappa_{t}^{ \pm}$evolve, the fill rate shape changes, while the market order activity rates $\lambda^{ \pm}$modulate the vertical scale. The maximum fill rate is achieved at $\delta=0$.

In the more stylized framework discussed in Chapter 3 , the rates $\lambda_{t}^{ \pm}$and $\kappa_{t}^{ \pm}$will all be stochastic processes to better represent the emperical features found in real data. Real data will be analysed in Chapter 6 with proper statistical analysis to support this claim.

The last notational component to this setup is to let $\left\{N_{t}^{-}\right\}_{0 \leq t \leq T}$ and $\left\{N_{t}^{+}\right\}_{0 \leq t \leq T}$ be counting processes corresponding to the MM's filled limit buy and sell quotes, respectively. If the MM is not allowed to execute market orders, then the current inventory is simply $q_{t}=N_{t}^{-}-N_{t}^{+}$(since all posted quotes are of unit volume) and the agent's cash reserve process is given by

$$
d X_{t}=\left(S_{t}+\delta_{t-}^{+}\right) d N_{t}^{+}-\left(S_{t}-\delta_{t-}^{-}\right) d N_{t}^{-}
$$

where $\delta_{t-}^{ \pm}$denotes the left limit of the process $\delta_{t}^{ \pm}$and $X_{0}=0$. Note that it is equivalent to use $S_{t}$ instead of $S_{t-}$ in the above equation since the trajectories of $S_{t}$ are continuous.

For a given $\mathcal{F}_{t}$ previsible strategy $\boldsymbol{\delta}_{t}$, the performance criterion of the MM (for $t \in[0, T]$ ) is

$$
J^{\delta}(t, x, s, q)=\mathbb{E}_{(t, x, s, q)}^{\mathbb{P}}\left[X_{T}^{\delta}+q_{T}^{\delta}\left(S_{T}-\gamma q_{T}^{\delta}\right)-\phi \int_{t}^{T}\left(q_{s}^{\delta}\right)^{2} d s\right]
$$

where $\phi>0$ and $\gamma>0$ are constants, and the processes $X_{t}$ and $q_{t}$ depend on the control $\boldsymbol{\delta}_{t}$. Going forward, this explicit dependence will often be supressed, along with the relevant probability measure $\mathbb{P}$. Finally, the subscript $(t, x, s, q)$ denotes that we are conditioning on the event $\left(t, X_{t}, S_{t}, q_{t}\right)=(t, x, s, q) \cdot{ }^{12}$

The parameter $\phi$ is an inventory management parameter, and its value depends on the

\footnotetext{
${ }^{12}$ More generally, we are conditioning on $\mathcal{F}_{t}$. However, we can simply condition on the relevant states at time $t$ since all processes are Markovian wrt $(\mathbb{F}, \mathbb{P})$.
} 
MM's inventory-aversion ${ }^{13}$ level. The last term in (2.2) is a penalty term that is associated with the MM holding non-zero inventory. The performance criterion is then the penalized market value of terminal holdings, where the penalty is related to how much inventory was held during the trading period. Some works, as in Avellaneda and Stoikov (2008), have perfomance criterion equal to expected utility (where the exponential utility function is the primary focus).

Although it is possible to have the integral of any non-negative convex penalty function, analytic tractability is immediately lost in such generality. The choice of squared inventory can stem for many different (and independent) sources. The analysis in Chapter 4 shows that such a penalty, even in a much more general framework, is equal to the intra-trade quadratic variation of the MM's market value process. On the other hand, Cartea et al. (2013) show that this exact penalty stems from ambiguity aversion in the drift of the midprice process.

The constant $\gamma$ is a market impact liquidation penalty for holding non-zero inventory at the terminal time $T$. Alternatively, this terminal penalty can be seen as stemming from the agent's aversion to holding inventory overnight. The explicit form of $\gamma q^{2}$ is due to the simplified assumption that the limit order book is flat. To liquidate a positive inventory of $q$, the average price per share received decreases linearly in $q$ and hence, the cost is quadratic in $q$. The case when covering a short position of $q$ is analogous.

The value function is then given by

$$
\Phi(t, x, s, q)=\sup _{\delta} J^{\delta}(t, x, s, q)
$$

where the supremum is taken over all $\mathcal{F}_{t}$ previsible functions $\boldsymbol{\delta}:[0, T] \times \Omega \longrightarrow \mathbb{R}^{2}$, and $J^{\delta}$ is defined in (2.2).

The goal of this problem is to determine the optimal strategy $\boldsymbol{\delta}_{t}^{*}$ that satisfies $\Phi=J^{\delta^{*}}$. The value function $\Phi$ is somewhat less interesting than the optimal strategy itself, even though its structure determines the optimal strategy.

\footnotetext{
${ }^{13}$ Some authors refer to this as risk-aversion. In this thesis, we emphasize that it is holding a large inventory that the high frequency trader is averse to.
} 


\subsection{The Solution}

To go from the definition of the value function to the HJB equation, we will employ a dynamic programming argument. To this end, consider a strategy $\widehat{\boldsymbol{\delta}}_{t}$ of the form

$$
\widehat{\boldsymbol{\delta}}_{u}= \begin{cases}\boldsymbol{\delta}_{u} & u \in[t, t+h] \\ \boldsymbol{\delta}_{u}^{*} & u \in(t+h, T]\end{cases}
$$

where $h>0, \boldsymbol{\delta}_{t}$ is an arbitrary control, and $\boldsymbol{\delta}_{t}^{*}$ is the optimal control. We proceed to evaluate the performance criterion given by the strategy $\widehat{\boldsymbol{\delta}}_{t}$.

$$
\begin{aligned}
J^{\widehat{\delta}}(t, x, s, q) & =\mathbb{E}_{(t, x, s, q)}\left[X_{T}^{\widehat{\delta}}+q_{T}^{\widehat{\delta}}\left(S_{T}-\gamma q_{T}^{\widehat{\delta}}\right)-\phi \int_{t}^{T}\left(q_{s}^{\widehat{\delta}}\right)^{2} d s\right] \\
& =\mathbb{E}_{(t, x, s, q)}\left[X_{T}^{\widehat{\delta}}+q_{T}^{\widehat{\delta}}\left(S_{T}-\gamma q_{T}^{\widehat{\delta}}\right)-\phi \int_{t+h}^{T}\left(q_{s}^{\delta^{*}}\right)^{2} d s-\phi \int_{t}^{t+h}\left(q_{s}^{\delta}\right)^{2} d s\right] \\
& =\mathbb{E}_{(t, \cdot)}\left[\mathbb{E}_{(t+h, \cdot)}\left\{X_{T}^{\widehat{\delta}}+q_{T}^{\widehat{\delta}}\left(S_{T}-\gamma q_{T}^{\widehat{\delta}}\right)-\phi \int_{t+h}^{T}\left(q_{s}^{\delta^{*}}\right)^{2} d s\right\}-\phi \int_{t}^{t+h}\left(q_{s}^{\delta}\right)^{2} d s\right] \\
& =\mathbb{E}_{(t, \cdot)}\left[\Phi\left(t+h, X_{t+h}^{\delta}, S_{t+h}^{\delta}, q_{t+h}^{\delta}\right)-\phi \int_{t}^{t+h}\left(q_{s}^{\delta}\right)^{2} d s\right] \\
& =\Phi(t, x, s, q)+\mathbb{E}_{(t, \cdot)}\left[\int_{t}^{t+h}\left\{\left(\partial_{t}+\mathcal{L}\right) \Phi\left(u, X_{u}^{\delta}, S_{u}^{\delta}, q_{u}^{\delta}\right) d u-\phi\left(q_{u}^{\delta}\right)^{2} d u\right\}\right]
\end{aligned}
$$

where the last equality is by Dynkin's Formula and the infinitesimal generator is given by

$$
\begin{aligned}
& \mathcal{L} \Phi(t, x, s, q)=\frac{1}{2} \sigma^{2} \partial_{s s} \Phi(t, x, s, q)+\lambda^{-} e^{-\kappa^{-} \delta^{-}}\left[\Phi\left(t, x-s+\delta^{-}, s, q+1\right)-\Phi(t, x, s, q)\right] \\
&+\lambda^{+} e^{-\kappa^{+} \delta^{+}}\left[\Phi\left(t, x+s+\delta^{+}, s, q-1\right)-\Phi(t, x, s, q)\right] .
\end{aligned}
$$

Taking supremum over all admissible controls $\boldsymbol{\delta}_{t}$ on the interval $[t, t+h]$ and dividing by 
$h$ yields

$$
\sup _{\delta} \mathbb{E}_{(t, \cdot)}\left[\frac{1}{h} \int_{t}^{t+h}\left\{\left(\partial_{t}+\mathcal{L}\right) \Phi\left(u, X_{u}^{\delta}, S_{u}^{\delta}, q_{u}^{\delta}\right) d u-\phi\left(q_{u}^{\delta}\right)^{2}\right\} d u\right]=0 .
$$

By taking the limit $h \downarrow 0$, the value function $\Phi$ then satisfies the following HJB equation with terminal condition

$$
\left\{\begin{array}{r}
\partial_{t} \Phi-\phi q^{2}+\frac{1}{2} \partial_{s s} \Phi+\lambda^{-} \sup _{\delta^{-}}\left\{e^{-\kappa^{-} \delta^{-}}\left[\Phi\left(t, x-s+\delta^{-}, s, q+1\right)-\Phi\right]\right\} \\
+\lambda^{+} \sup _{\delta^{+}}\left\{e^{-\kappa^{+} \delta^{+}}\left[\Phi\left(t, x+s+\delta^{+}, s, q-1\right)-\Phi\right]\right\}=0 \\
\Phi(T, \cdot)=x+q(s-\gamma q)
\end{array}\right.
$$

where the "." notation in $\Phi(T, \cdot)$ is used to suppress arguments and appears throughout this thesis.

We can apply the ansatz $\Phi=x+q s+g(t, q)$ to reduce (2.7) to the much simpler form:

$$
\left\{\begin{aligned}
\partial_{t} g(t, q)-\phi q^{2}+\lambda^{-} \sup _{\delta^{-}}\left\{e^{-\kappa^{-} \delta^{-}}\left[\delta^{-}+g(t, q+1)-g(t, q)\right]\right\} & \\
+\lambda^{+} \sup _{\delta^{+}}\left\{e^{-\kappa^{+} \delta^{+}}\left[\delta^{+}+g(t, q-1)-g(t, q)\right]\right\} & =0 \\
g(T, q) & =-\gamma q^{2}
\end{aligned}\right.
$$

The optimal strategy can be found in feedback control form (i.e. as a function of the value function $\Phi$ or, equivalently, $g$ ) by finding the maximizer of the supremum terms in (2.8). If we differentiate in $\delta^{ \pm}$and set the resulting expression equal to 0 , we find the global maximum ${ }^{14}$ is obtained at

$$
\delta_{t}^{ \pm, *}=\frac{1}{\kappa^{ \pm}}-[g(t, q \mp 1)-g] .
$$

If we substitute this optimal control back into (2.8), the following non-linear equation for $g$ is obtained:

$$
\partial_{t} g-\phi q^{2}+\frac{\lambda^{-}}{e \kappa^{-}} e^{\kappa^{-}[g(t, q+1)-g]}+\frac{\lambda^{+}}{e \kappa^{+}} e^{\kappa^{+}[g(t, q-1)-g]}=0
$$

\footnotetext{
${ }^{14}$ One would also need to check second order and boundary/limiting conditions, but these details are omitted.
} 
At this point, there are many methods for solving (2.10). We briefly outline three of them below.

1. Perform an asymptotic expansion in $\phi$. This method is similar to the solution to the problem in Chapter 3.

2. Impose upper and lower bound constraints on $q$, and then proceed to solve (2.10) numerically using an explicit scheme backwards in time. This method is similar to the solution to the problem in Chapter 4.

3. Impose upper and lower bound constraints on $q$, and then attempt to find an exact solution to the resulting finite system of ordinary differential equations (ODEs). This method is similar to the solutions to the problems posed in Cartea and Jaimungal $(2013 b)^{15}$ and Guéant et al. $(2013)^{16}$.

The numerical solution is obtained via an explicit numerical scheme, as described above in item 2. Since the purpose of this chapter is to explore the setup of the market making problem and the qualitative features of the optimal control, the explicit details regarding this numerical solution are omitted.

\subsection{Features of the Optimal Strategy}

We further impose that the admissible controls in (2.3) be restricted to only non-negative controls (that is, $\mathbb{R}_{+}^{2}$-valued functions). This additional constraint is a financial one: We do not want the MM to submit limit quotes that cross up the LOB $^{17}$ (as these are akin to market orders which will be addressed in Section 2.4).

Figure 2.3 depicts the behaviour of the optimal posting depth for buy limit orders (the analysis for sell limit orders is analogous due to the model's inherent symmetry). Notice for a fixed inventory-aversion level $\phi$, the MM posts deeper into the LOB when she is holding a large positive inventory. Likewise, the more short she is, the closer the bid quote will be to the midprice as she is more eager to acquire the asset and willing to do so for less spread. This induces her inventory to mean-revert to zero, despite that requirement not being part of the initial setup of the problem.

\footnotetext{
${ }^{15}$ The choice of ansatz for $\Phi$ is slightly different.

${ }^{16}$ The objective function in this paper maximizes expected utility instead of expected penalized profits.

${ }^{17} \mathrm{~A}$ LOB is deemed to be crossed if there exists some subset of quotes that can be executed against each other. Typical cases are concerned with subsets of size two, but some brokerages will search for crosses that involve more than two quotes.
} 


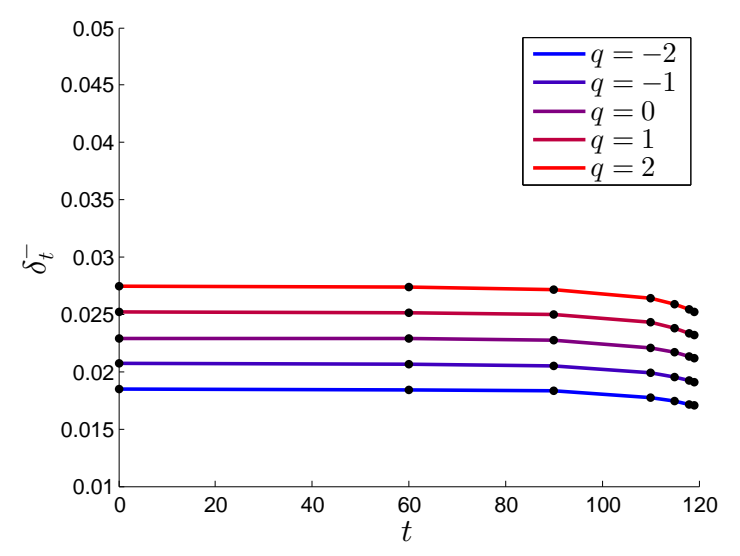

(a) $\phi=1$

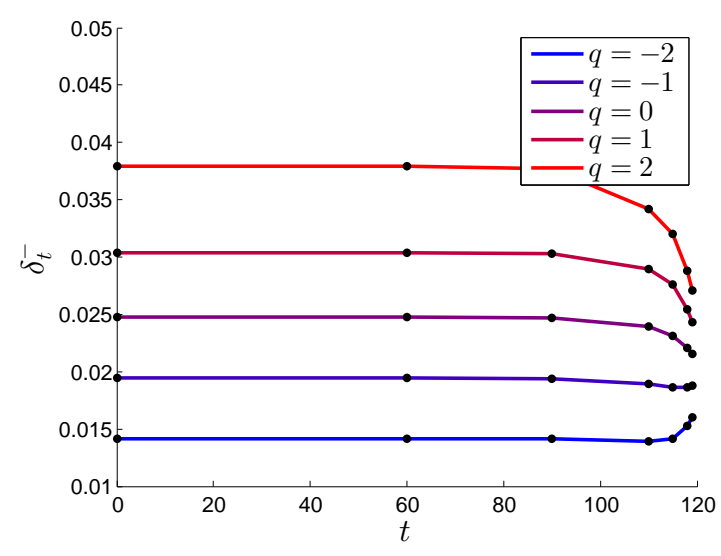

(b) $\phi=5$

Figure 2.3: Optimal posting depth of limit buy quotes $\delta_{t}^{-}$for various inventory levels $q$ versus time with terminal investment horizon $T=120$ and maximum inventory size, for the purpose of numerically solving (2.10), of 40 units (long or short).

When we compare the behaviour of two MMs with different inventory-aversion levels $(\phi=1$ versus $\phi=5)$, we see that the distinction in behaviour for different inventory levels becomes much more pronounced for the MM that is more inventory-averse (as depicted in Figure 2.3(b)). This is due to the fact that the more inventory-averse the $\mathrm{MM}$ is, the stronger the desire to get back to a flat position $(q=0)$. Hence, when this MM is long, far more compensation is required to accumulate yet another unit of the asset, so $\delta_{t}^{-}$is large (i.e. the bid quote is posted deep in the LOB). Similarly, this $\mathrm{MM}$ is even more desperate to cover short positions, so $\delta_{t}^{-}$quickly becomes small when inventory begins to accumulate. Different rates of mean reversion of the MM's inventory will be investigated in Section 3.5.3.

Finally, the behaviour we observe in $\delta_{t}^{-}$when $t$ approaches $T=120$ is dictated by the size of the market impact parameter $\gamma$. As demonstrated in Cartea and Jaimungal (2013b), when $\gamma=0$, these curves (one for each $q$ ) converge to a point as the MM knows that she can liquidate at the midprice at time $T$, so she attempts to accumulate more of the asset to collect the spread. When $\gamma$ is large, the curves will diverge from each other as the cost of terminal market impact is extremely high, so the trader desperately tries to cover any short position while posting deep bid quotes to be sure to not accumulate more inventory. This effect of terminal market impact detracts from the scope of this chapter, so we refer the reader to Cartea and Jaimungal (2013b) for more details.

A simulation study is performed on 5 minutes of trading ( $T=300$ seconds) for 2,500 total simulations. The other model parameters are given in Table 2.1. See Figure 2.4(a) for the effect that the inventory-aversion level has on the terminal profit and loss (PnL) 


\begin{tabular}{cc}
\hline \hline Parameter & Value \\
\hline$\sigma$ & 0.01 \\
$S_{0}$ & 100 \\
$\gamma$ & 0.001 \\
$\lambda^{-}$ & 1.2 \\
$\lambda^{+}$ & 1.0 \\
$\kappa^{-}$ & 50 \\
$\kappa^{+}$ & 55 \\
$T$ & 300 \\
\hline \hline
\end{tabular}

Table 2.1: Model parameters used in the simulation study for the market making problem.

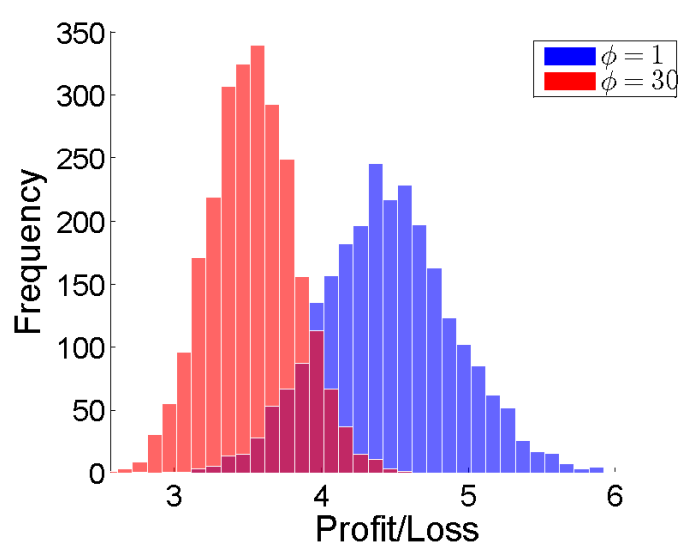

(a) Distribution of PnL

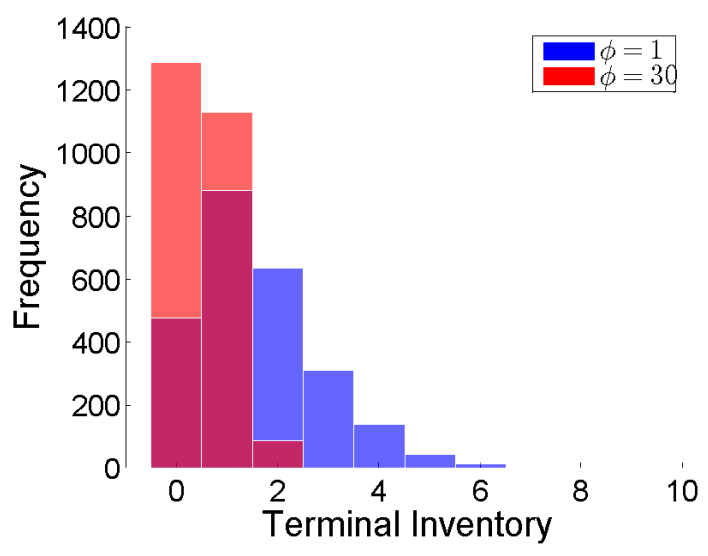

(b) Distribution of the terminal inventory $\left|q_{T}\right|$

Figure 2.4: Distributional properties associated with the optimal strategy for various inventory-aversion levels, $\phi$.

of the MM. Observe that the more inventory-averse the MM is, the lower her mean PnL is. However, the more inventory-averse MM also faces a PnL distribution with smaller variance. This is directly analogous to the classical risk-return trade-off in portfolio theory.

Furthermore, Figure 2.4(b) shows that the distribution of absolute terminal inventory, $\left|q_{T}\right|$, becomes closer to zero as the MM becomes more inventory-averse. This is in line with the financial intuition that inventory-averse traders prefer to hold smaller inventories than less inventory-averse ones. 


\subsection{The Inclusion of Market Orders}

In this section, we modify the market making problem posed in Section 2.1 to allow the MM to be aggressive and execute market orders. ${ }^{18}$ We wish to investigate what effect, if any, this has on the trading behaviour of MM and how it affects her PnL. The agent's optimization problem now consists of a continuous control and an impulse control. More specifically, it consists of $\boldsymbol{\delta}=\left(\delta^{-}, \delta^{+}\right)$and the double sequence of stopping times and impulses $(\boldsymbol{\tau}, \boldsymbol{\varsigma})=\left(\tau_{1} \leq \tau_{2} \leq \ldots ; \varsigma_{1}, \varsigma_{2}, \ldots\right)$ at which the agent executes MOs, where $\tau_{k}$ is an $\mathcal{F}_{t}$ stopping time (for all $k$ ), $\varsigma_{k}=1$ if the $k^{\text {th }}$ market order is a buy, and $\varsigma_{k}=-1$ if it is a sell.

Most electronic exchanges charge a fee for taking liquidity from the market, hence, when the agent executes a market order, here, we will charge her an additional transaction fee. For the purposes of illustration, this fee is taken to be $\epsilon=0.01$ for this section only. This fee can also be interpreted as the half-spread in a microstructure model that has a bid-offer spread.

We will denote the agent's buy and sell MO submissions by the counting processes $\left\{\widetilde{M}_{t}^{+}\right\}_{0 \leq t \leq T}$ and $\left\{\widetilde{M}_{t}^{-}\right\}_{0 \leq t \leq T}$, respectively. The MM's cash process then satisfies the SDE

$$
d X_{t}=\left(S_{t}+\delta_{t-}^{+}\right) d N_{t}^{+}-\left(S_{t}-\delta_{t-}^{-}\right) d N_{t}^{-}+\left(S_{t}-\epsilon\right) d \widetilde{M}_{t}^{-}-\left(S_{t}+\epsilon\right) d \widetilde{M}_{t}^{+}
$$

and the inventory process $q_{t}$ is given by $q_{t}=N_{t}^{-}+\widetilde{M}_{t}^{+}-N_{t}^{+}-\widetilde{M}_{t}^{-}$.

Notice that the supremum in Equation (2.3) will now be taken over $(\boldsymbol{\delta}, \boldsymbol{\tau}, \boldsymbol{\varsigma})$ to reflect the fact that this is a combined stochastic control and impulse control problem. Similar to Section 2.2, we derive the HJB quasi-variational inequality (QVI) via a dynamic programming argument.

To this end, fix $t \in[0, T)$ and define the impulse control $\widehat{\boldsymbol{\tau}}=\left(\tau_{1} \leq \tau_{2}^{*} \leq \tau_{3}^{*} \leq \ldots\right)$ where $\tau_{1} \in[t, T]$ is an arbitrary stopping time and $\tau_{2}^{*}, \tau_{3}^{*}, \ldots$ are optimal stopping times. We then define the continuous control $\widehat{\boldsymbol{\delta}}$ as

$$
\widehat{\boldsymbol{\delta}}_{u}= \begin{cases}\boldsymbol{\delta}_{u} & u \in\left[t, \tau_{1} \wedge(t+h)\right] \\ \boldsymbol{\delta}_{u}^{*} & \text { o.w. }\end{cases}
$$

where $h>0, \boldsymbol{\delta}_{t}$ is an arbitrary control, and $\boldsymbol{\delta}_{t}^{*}$ is the optimal control. Following the same

\footnotetext{
${ }^{18}$ See Horst and Naujokat (2014) for the inclusion of market orders when trading in illiquid markets.
} 
logic as in Section 2.2, we arrive at the analogue to Equation (2.6):

$$
\sup _{\delta, \tau, \boldsymbol{\varsigma}} \mathbb{E}_{(t, \cdot)}\left[\frac{1}{h} \int_{t}^{\tau_{1} \wedge(t+h)}\left\{\left(\partial_{t}+\mathcal{L}\right) \Phi(u, \cdot) d u-\phi\left(q_{u}\right)^{2}+\mathcal{M} \Phi(u, \cdot)\right\} d u\right]=0
$$

where $\mathcal{M}$ is the impulse operator responsible for the agent's submission of market orders and is given by

$$
\mathcal{M} \Phi(u, x, s, q)=\sum_{i: \tau_{i}=u} \sup _{\varsigma_{i}}\left[\Phi\left(u, x-\varsigma_{i} \cdot s-\epsilon, s, q+\varsigma_{i}\right)-\Phi(u, x, s, q)\right],
$$

and $\mathcal{L}$ is defined in (2.5). Recall that $\varsigma_{i}=1$ when the $i^{\text {th }}$ market order is a buy and $\varsigma_{i}=-1$ when it is a sell.

When taking $h \downarrow 0$ in Equation (2.12), we consider the two cases: $\tau_{1}=t$ and $\tau_{1}>t$. Note that the event $\left\{\tau_{1}=t\right\}$ is $\mathcal{F}_{t}$ measurable and therefore we know at time $t$ whether $\tau_{1}=t$ or $\tau_{1}>t$. If $\tau>t$, then the impulse term in (2.12) is zero (by definition) and we obtain something similar to Equation (2.6). If $\tau_{1}=t$, then the integral in (2.12) is zero and only the impulse term remains. Because we are taking supremum over all continuous controls and impulses, we see that the maximum of these terms must be zero. Analogous to Equation (B.4), the resulting HJB equation that the value function $\Phi$ satisfies will now be a QVI and is given by

$$
\left\{\begin{array}{r}
\max \left[\partial_{t} \Phi-\phi q^{2}+\frac{1}{2} \partial_{s s} \Phi+\lambda^{-} \sup _{\delta^{-}}\left\{e^{-\kappa^{-} \delta^{-}}\left[\Phi\left(t, x-s+\delta^{-}, s, q+1\right)-\Phi\right]\right\}\right. \\
+\lambda^{+} \sup _{\delta^{+}}\left\{e^{-\kappa^{+} \delta^{+}}\left[\Phi\left(t, x+s+\delta^{+}, s, q-1\right)-\Phi\right]\right\} \\
\Phi(t, x-s-\epsilon, s, q+1)-\Phi ; \\
\Phi(t, x+s-\epsilon, s, q-1)-\Phi]=0 \\
\Phi(T, \cdot)=x+q(s-\gamma q)
\end{array}\right.
$$

Observe the addition of the second and third terms in the maximum in (2.13). The second term corresponds to the submission of a market buy order and the cash process decreases by $s+\epsilon$. Similarly, the third term corresponds to a market sell for the price of $s-\epsilon$.

One can still apply the simplifying ansatz $\Phi=x+q s+g(t, q)$. Interestingly enough, the optimal control in feedback control form, $\boldsymbol{\delta}$, in the continuation region is still given by (2.9). The function $g(t, q)$ in this case is clearly different though. Due to the HJBQVI in (2.13) being the maximum of three components, it appears that we have lost analytic 


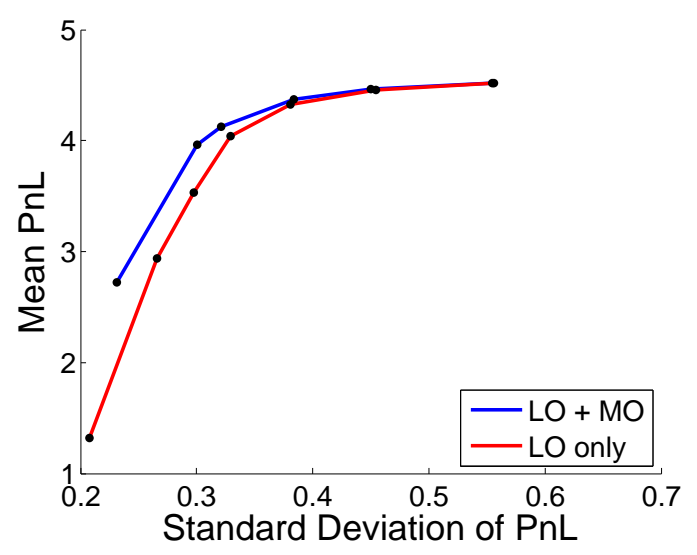

(a) Mean PnL vs Std Dev PnL

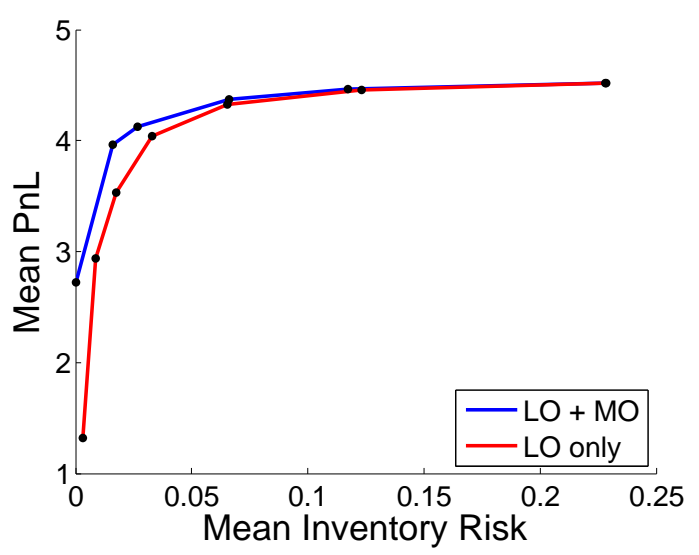

(b) Mean PnL vs Mean Inventory Risk

Figure 2.5: Performance of strategy for various $\phi$, ranging from 0.3 to 200, with and without market orders.

tractibility of $g$ (even in an asymptotic regime). Of course, one can resort to an explicit numerical scheme and solve (2.13) backwards in time.

As carried out in Section 2.3, here we perform a simulation study and investigate the performance of various inventory-aversion levels $\phi$. Figure 2.5 shows the efficient frontiers in both the limit order (LO) only and LO + MO cases. The range of $\phi$ is from 0.3 to 200 and recall that the other model parameters are given in Table 2.1. The least inventoryaverse trader obtains the mean PnL in the upper right corner of these plots, but also has both the highest $\mathrm{PnL}$ variance and mean inventory risk ${ }^{19}$. As the MM becomes more inventory-averse (increases $\phi$ ), the performance of the strategy moves towards the points in the bottom left corner. These strategies have less risk, but yield less overall profits.

It is clear that the optimal strategy without MOs is an admissible strategy in the case when MOs are allowed. Hence, the value function that allows MOs must always dominate the value function that allows LOs only. This is also evident from the efficient frontiers in Figure 2.5 and demonstrates the value of market orders to the market maker.

The number of market orders the MM executes will depend on how inventory-averse she is (i.e. how willing she is to hold inventory instead of paying a fee to take liquidity from the market to flatten the position prior to time $T$ ). Figure 2.6 shows the distribution of the number of executed market orders for various $\phi$. Observe that the more inventory-averse the MM is, the more she executes market orders. As the inventory penalty parameter $\phi$

\footnotetext{
${ }^{19}$ Inventory risk is defined as: $\sigma^{2} \int_{0}^{T} q_{s}^{2} d s$, and represents how much risk was taken on over the lifetime of the strategy. This concept, and its multiple asset variant, will be elaborated on in Section 4.4.1.
} 


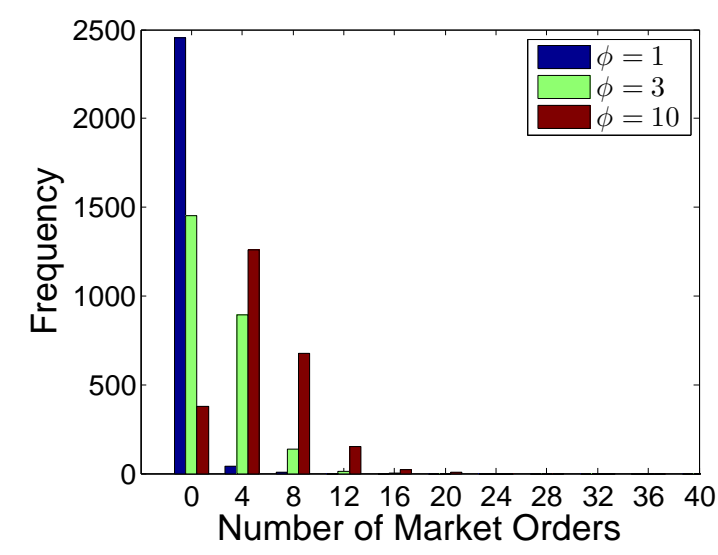

(a) Market Buy Orders

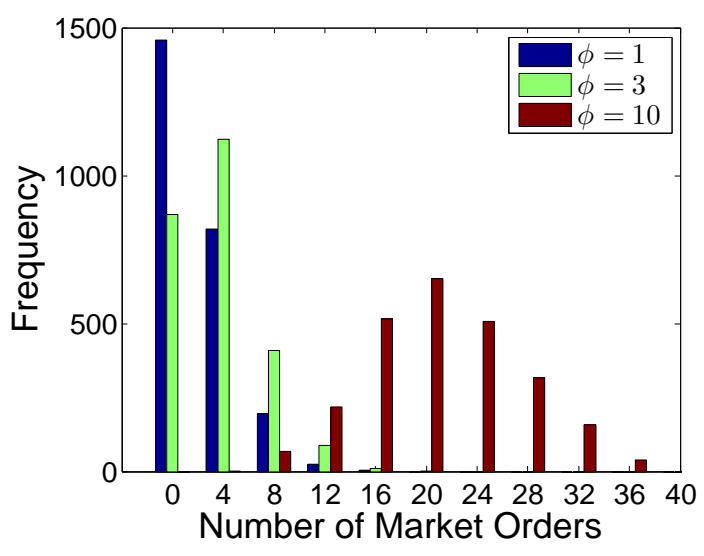

(b) Market Sell Orders

Figure 2.6: Distribution of the number of executed marker orders for various $\phi$.

goes to zero (i.e. the MM becomes risk neutral), she chooses to never execute MOs since she does not mind holding large inventories. Hence, the strategy that includes MOs is the same as the LO only strategy and this corresponds to the upper right region of the efficient frontiers of Figure 2.5. However, for inventory-averse MMs, there is certainly value in the ability to execute MOs.

The larger number of sell MOs executed (compared to buy MOs) is due to the slight asymmetry in the the chosen parameters in Table 2.1. $\lambda^{-}>\lambda^{+}$signifies that there are more incoming market sell orders, making fills on the bid side occur more often relative to fills on the offer side. $\kappa^{-}<\kappa^{+}$signifies that there are more agents that have limit sell quotes posted, meaning that the LOB is heavier on the limit sell side, making it harder to get filled there relative to the limit buy side. Both of these effects combined cause the MM to execute more market sell orders.

Figure 2.7 shows the maximum (absolute) inventory held by the MM during the lifetime of the strategy. Again, notice that the ability to execute market orders helps the MM directly control how large her inventory gets. When $\phi=1$, the MM makes use of MOs to prevent her inventory from becoming larger than $q=7$ (or smaller than $q=-7$ ). Increasing the inventory aversion parameter to $\phi=3$, the MM makes heavier use of MOs to prevent her inventory from becoming larger than $q=4$. Whereas in the LO only case, she must post her limit buy quote deeper in the LOB and demands more spread in exchange for accumulating another unit of the asset. This causes her to be filled less often and has a direct impact on PnL.

The model and results presented in this chapter have now set the stage for the more complex algorithmic trading problems in the next two chapters: Chapter 3 introduces 


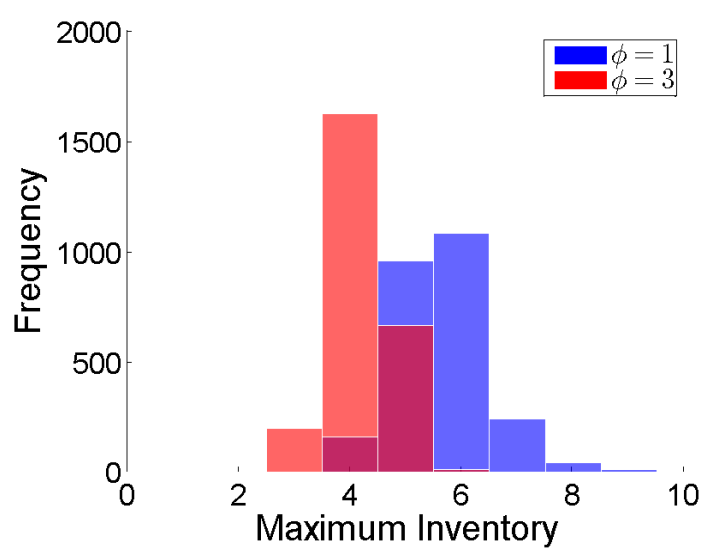

(a) LO only

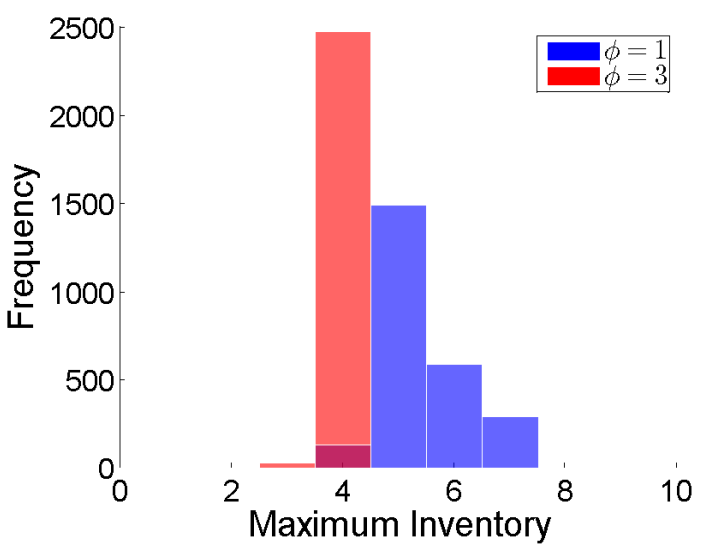

(b) $\mathrm{LO}+\mathrm{MO}$

Figure 2.7: Distribution of $\max _{0 \leq t \leq T}\left\{\left|q_{t}\right|\right\}$ for various $\phi$.

stochastic MO arrival rate processes $\lambda_{t}^{ \pm}$and stochastic midprice $\alpha_{t}$ to incorporate adverse selection into our microstructure model, while Chapter 4 extends this setup to multiple assets where an investor seeks to both make markets and profit from short term statistical arbitrage opportunites. 


\section{Chapter 3}

\section{Market Making in a Single Asset}

\subsection{Introduction}

There is little evidence on the source of high frequency (HF) market making (MM) profits, but the picture that is emerging is that price anticipation and short-term price deviations from the fundamental value of the asset are important drivers of profits. On the other hand, from the classical microstructure literature on adverse selection (see, e.g., O'Hara (1998)), we also know that strategies that do not include a buffer in their LOs to cover adverse selection costs or that strategically post deeper in the book to avoid being picked off, may see their accumulated profits dwindle as a consequence of trading with other market participants who possess private or better information. In the long term, high frequency traders (HFTs) who are not able to incorporate short-term price predictability in their optimal HF market making strategies, as well as account for adverse selection costs, are very likely to be driven out of the market.

The goal of this chapter is to develop a particular dynamic HF trading strategy based on optimal postings and cancellations of limit orders to maximize expected terminal wealth over a fixed horizon $T$ while penalizing inventories, which will be made mathematically precise in Section 3.5.1. The HFT we characterize here can be thought of as an ultrafast market maker where the trading horizon $T$ is at most one trading day, all her limit orders are cancelled a short time later if not filled, and inventories are optimally managed (to maximize expected penalized terminal wealth) and drawn to zero by $T .{ }^{20}$ Early work on

\footnotetext{
${ }^{20}$ HFTs closely monitor their exposure to inventories for many reasons. For example, HFTs' own risk controls or regulation do not allow them to build large (long or short) positions; the HFT is capital constrained and needs to post collateral against her inventory position. Moreover, we remark that there is no consensus on characterizing HFTs as market makers because some stakeholders and regulatory
} 
optimal postings by a securities dealer is that of Ho and Stoll (1981), and more recently Avellaneda and Stoikov (2008) studied the optimal HF submission strategies of bid and ask limit orders.

Intuitively, our dynamic high frequency trading (HFT) strategy maximizes the expected profits resulting from roundtrip trades by specifying how deep on the sell and buy sides the limit orders are placed in the limit order book (LOB). The HFT strategy is based on predictable short-term price deviations and managing adverse selection risks that result from trading with counterparties that may possess private or better information. Clearly, the closer the limit orders are to the best bid and best offer, the higher the probability of being executed, but the expected profits from a roundtrip are also lower, and adverse selection costs are higher.

Accumulated inventories play a key role throughout the entire strategy we develop. Optimal postings control for inventory risks by sending quotes to the LOB which induce mean reversion of inventories to an optimal level and by including a state dependent buffer to cover or avoid expected adverse selection costs, as will be discussed in Sections 3.5.2, 3.5.3, and 3.6. For example, if the probability of the next market order being a buy or sell is the same, and inventories are positive, then the limit sell orders are posted closer to the best ask, and the buy orders are posted further away from the best bid so that the probability of the offer being lifted is higher than the bid being hit. Furthermore, as the dynamic trading strategy approaches the terminal time $T$, orders are posted nearer to the midquote to induce mean-reversion to zero in inventories, which avoids having to post collateral overnight and bearing inventory risks until the market opens the following day. Similarly, if the HF trading algorithm detects that limit orders on one side of the LOB are more likely to be adversely selected, then these limit orders are posted deeper into the book in anticipation of the expected adverse selection costs. An increase in adverse selection risk could be heralded by market orders becoming more one-sided as a consequence of the activity of traders acting on superior or private information who are sending one-directional market orders.

As part of the model we develop here, we propose a reduced-form model for the intensity of the arrival of market sell and buy orders. The novelty we introduce is to assume that market orders arrive in two types. The first type of orders are influential orders which excite the market and induce other traders to increase the number of market orders they submit. For instance, the arrival of an influential market sell order increases the probability of observing another market sell order over the next time step and also increases (to a lesser extent) the probability of a market buy order to arrive over the next time step. On the other hand, when non-influential orders arrive, the intensity of

authorities point out that their holding periods are too short to consider them as such; see, for example, European Commission (2010). 
the arrival of market orders does not change. This reflects the existence of trades that the rest of the market perceives as not conveying any information that would alter their willingness to submit market orders. In our model, we also incorporate the arrival of public news as a state variable that increases the intensity of market orders. In this way, our model for the arrival of market orders is able to capture trade clustering which can be one-sided or two-sided and allow for the activity of trading to show the positive feedback that algorithmic trades seem to have brought to the market environment. Multivariate Hawkes processes have recently been used in the financial econometrics literature to model clustering in trade arrival and changes in the LOB; see, e.g., Large (2007), Bowsher (2007) and Toke (2011). However, this work is the first to incorporate such effects into optimal control problems related to algorithmic trading (AT).

In our model, the arrival of trades also affects the midprice and the LOB. The arrival of market orders is generally regarded as an informative process because it may convey information about subsequent price moves and adverse selection risks. ${ }^{21}$ Here we assume that the dynamics of the midprice of the asset are affected by short-term imbalances in the number of influential market sell and buy orders; in particular, these imbalances have a temporary effect on the drift of the midprice. The arrival of good and bad news have a similar effect.

Moreover, in our model the arrival of influential orders has a transitory effect on the shape of both sides of the LOB. More specifically, since some market makers anticipate changes in the intensity of both the sell and buy market orders, the shape of the buy and sell sides of the book will also undergo a temporary change due to market makers repositioning their limit orders in anticipation of the increased expected market activity and adverse selection risk.

We test our model using simulations where we assume different types of HFTs who are mainly characterized by the quality of the information that they are able to process and incorporate into their optimal postings. We show that those HFTs who incorporate predictions of short-term price deviations in their strategy will deliver positive expected profits. At the other extreme, we have the HFTs who are driven out of the market because their limit orders are picked off by better informed traders (see Carmona and Webster (2012a) for an order book model that distinguishes between informed and uninformed traders) and cannot profit from directional strategies which are also based on short-lived predictable trends. We also show that in between these two cases, those HFTs who cannot execute profitable directional strategies (and are systematically being picked off)

\footnotetext{
${ }^{21}$ For instance, periods where the number of market buy orders is much higher than the number of market sell orders could be regarded as times where informed traders have a private signal and are adversely selecting market makers who are unaware that they are providing liquidity at a loss; see Easley and O'Hara (1992).
} 
can stay in business if they exert tight controls on their inventories. In our model, these controls imply a higher penalty on their inventory position which pushes the optimal limit orders further away from the midprice so the chances of being picked off by other traders are considerably reduced.

In Section 6.3, we also test these strategies against real data, where similar results are found. This analysis is postponed to Chapter 6 (a chapter dedicated to applications to real data) as it ties in nicely with applications of the theory from Chapter 5 as well.

\subsection{Arrival of Market Orders and Price Dynamics}

The HF trading strategy we develop here is designed to profit from the realized spread where we allow the HFT to build inventories. To this end, before we formalize the HFT's optimization problem, we require a number of building blocks to capture the most important features of the market dynamics. ${ }^{22}$ Since the HFT maximizes expected terminal wealth over a finite horizon $T$, while being penalized for holding large inventories, and she is continuously repositioning buy and sell limit orders (LOs), the success of the strategy depends on optimally picking the "best places" on the bid and offer side of the LOB which requires us to model (i) the dynamics of the fundamental value of the traded stock, (ii) the arrival of market buy and sell orders, and (iii) how market orders (MOs) cross the resting orders in the LOB. In this section we focus on (i) and (ii), in Section 3.3 we discuss (iii) and after that we present the formal optimal control problem that the HFT solves.

\subsubsection{Price Dynamics}

We assume that the midprice (or fundamental price) of the traded asset follows

$$
d S_{t}=\left(v+\alpha_{t}\right) d t+\sigma d W_{t}
$$

where $\left\{W_{t}\right\}_{0 \leq t \leq T}$ is a $\mathbb{P}$-standard Brownian Motion, and $S_{0}>0$ and $\sigma>0$ are constants. ${ }^{23}$ The drift of the midprice is given by a long-term component $v$ and by $\alpha_{t}$ which

\footnotetext{
${ }^{22}$ Although we focus on an HF trading market making algorithm, the framework we develop here can be adapted for other types of AT strategies.

${ }^{23}$ Unless otherwise stated, all random variables and stochastic processes in this chapter are defined on the completed, filtered probability space $\left(\Omega, \mathcal{F}_{T}, \mathbb{F}, \mathbb{P}\right)$ with filtration $\mathbb{F}=\left\{\mathcal{F}_{t}\right\}_{0 \leq t \leq T}$ and where $\mathbb{P}$ is the real-world probability measure. What generates the filtration will be defined precisely in Section 3.5. Simply put, it will be generated by the Brownian motions $W_{t}$ and $B_{t}$ (introduced later), counting
} 
is a predictable zero-mean reverting component that represents stochastic short-term deviations from $v$. Since we are interested in HF trading, our predictors are based on order flow information where we allow for feedback between market order events and shortterm-alpha. In the rest of this chapter, we assume that $v=0$ because the HF strategies we develop are for very short-term intervals.

Section 3.4 gives details of the dynamics of the process $\alpha_{t}$. This element of the model plays a key role in the determination of the HF strategies we develop because it captures different features that we observe in the dynamics of the midprice. For instance, it captures the price impact that some orders have on the midprice as a result of: the arrival of news, a burst of activity on one or both sides of the market, orders that eat into the LOB, etc. Furthermore, we also know that a critical component of HF trading is the ability that HFTs have to predict short-term deviations in prices so that they make markets by taking advantage of directional strategies based on short-term predictions (i.e., they are able to predict short-term-alpha) while at the same time allowing them to reposition stale quotes or submit new quotes to avoid being picked off by other market participants trading on short-term-alpha, i.e., avoid being adversely selected.

\subsubsection{Self-Exciting Incoming Market Order Dynamics}

Markets tend to follow an intraday pattern. Usually after the market opens and before the market closes, there is more activity than during the rest of the day. Another prominent feature is the clustering of MO arrivals. Figure 3.1 shows the historical intensity of trade arrival, buy and sell, for IBM over a two minute period (starting at 3.30pm, February 1 2008). The historical intensities are calculated by counting the number of buy and sell market orders over the last 1 second. The fitted $\rho=1$ intensities are computed using our model (see Equation (3.2)) under the specific assumption that all trades are influential; see Appendix E.1 for more details. Although the discussion regarding the fit of the full model will be deferred to Section 5.1 (where the figure is repeated), we would like to bring the reader's attention to the improvement in fit over the $\rho=1$ case.

From these figures, we observe that market orders may arrive in clusters and that there are times when the markets are mostly one-sided (for instance, there are periods where trading is more active on the buy side than on the sell side, and vice-versa) and that these bursts of activity die out rather quickly and revert to just a couple events per second. The statistical justification for these claims are outlined in detail in Section 6.1.

processes corresponding to buy/sell market and filled limit orders, news events, the indicator of whether a trade is influential or not, and the midprice's drift process. 


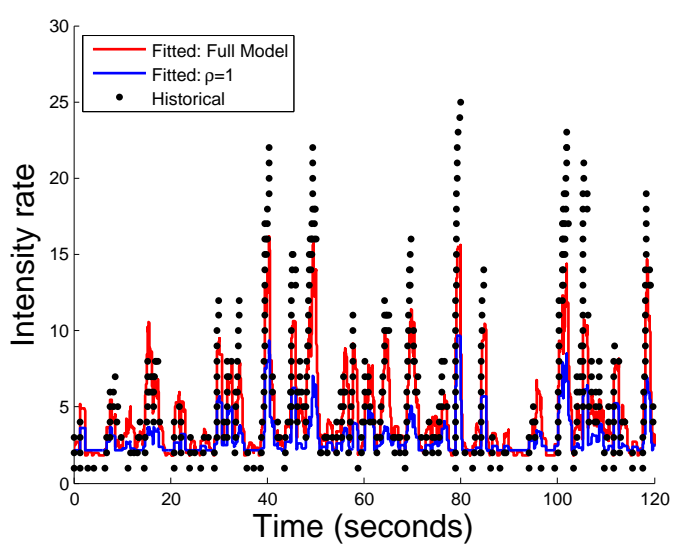

(a) Arrival of IBM market buy orders

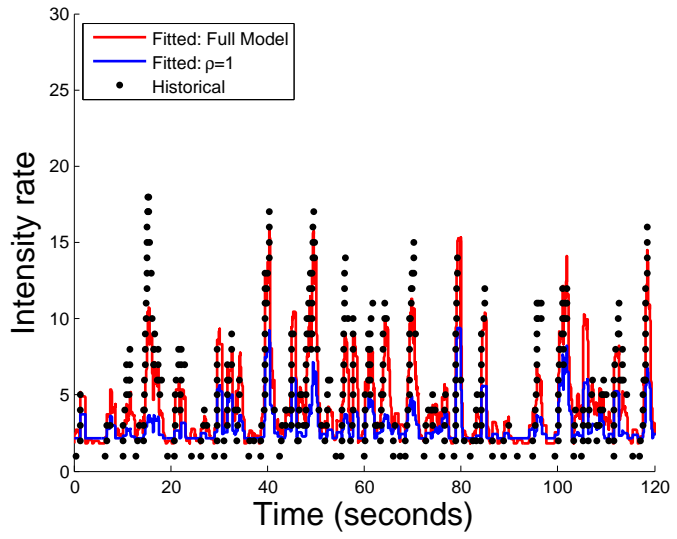

(b) Arrival of IBM market sell orders

Figure 3.1: IBM market orders. market orders. Historical running intensity versus smoothed fitted intensity using a 1 second sliding window for IBM for a 2 minute period, between 3:30pm and 3:32pm, February 1, 2008.

Why are there bursts of activity on the buy and sell sides? It is difficult to link all these short-lived increases in the levels of activity to the arrival of news. One could argue that trading algorithms, including $\mathrm{HF}$, are also responsible for the sudden changes in the pace of the market activity, including bursts of activity in the LOB, and most of the time these algorithms act on information which is difficult to link to public news. Thus, here we take the view that some market orders generate more trading activity in addition to the usual effect of news increasing the intensity of market orders.

In our model, market orders arrive in two types. The first are influential orders which excite the state of the market and induce other traders to increase their trading activity. As discussed in Section 3.4, these MOs can also be interpreted as originating from an agent with superior information, highlighting the the presence of asymmetric information in the market. ${ }^{24}$ We denote the total number of arrivals of influential sell/buy market orders up to (and including) time $t$ by the processes $\left\{\bar{M}_{t}^{-}, \bar{M}_{t}^{+}\right\}_{0 \leq t \leq T}$. The second type of orders are non-influential orders. These are viewed as arising from players who do not excite the state of the market. We denote the total number of arrivals of non-influential sell/buy market orders up to (and including) time $t$ by the processes $\left\{\widetilde{M}_{t}^{-}, \widetilde{M}_{t}^{+}\right\}_{0 \leq t \leq T}$. Note that the type indicator of an order is not observable. Rather, all one can observe is whether the market became more active after that trade. Therefore we assume that, conditional on the arrival of a market order, the probability that the trade is influential is constant and denoted by $\rho \in[0,1]$.

\footnotetext{
${ }^{24}$ Although this interpretation contradicts the Efficient Market Hypothesis, adverse selection is such a prominent feature in the market that we are willing to dismiss this hypothesis.
} 
Clearly, public bad (good) news increases the sell (buy) activity, but what is not clear is whether market participants always interpret news in the same way. If there is disagreement in how to interpret news or news is ambiguous, then both sides of the market will show an increase in the intensity of buy and sell market orders. ${ }^{25}$

Thus, we model the intensity of sell, $\left\{\lambda_{t}^{-}\right\}_{0 \leq t \leq T}$, and buy, $\left\{\lambda_{t}^{+}\right\}_{0 \leq t \leq T}$, market orders by assuming that they solve the coupled system of stochastic differential equations (SDEs).

Assumption 3.2.1. The rate of arrival of market sell/buy orders $\boldsymbol{\lambda}_{t}=\left(\lambda_{t}^{-}, \lambda_{t}^{+}\right)$solves the coupled system of SDEs

$$
\left\{\begin{array}{l}
d \lambda_{t}^{-}=\beta\left(\theta-\lambda_{t}^{-}\right) d t+\eta d \bar{M}_{t}^{-}+\nu d \bar{M}_{t}^{+}+\tilde{\eta} d Z_{t}^{-}+\tilde{\nu} d Z_{t}^{+}, \\
d \lambda_{t}^{+}=\beta\left(\theta-\lambda_{t}^{+}\right) d t+\eta d \bar{M}_{t}^{+}+\nu d \bar{M}_{t}^{-}+\tilde{\eta} d Z_{t}^{+}+\tilde{\nu} d Z_{t}^{-}
\end{array}\right.
$$

where $\left\{Z_{t}^{ \pm}\right\}_{0 \leq t \leq T}$ are Poisson processes (independent of each other and all other processes), with constant activity rate $\mu^{ \pm} \geq 0$, which represent the total amount of good and bad news that have arrived until time $t$, and recall that $\bar{M}_{t}^{+}$and $\bar{M}_{t}^{-}$are the total number of influential buy and sell orders up until time $t$. Moreover, $\beta, \theta, \eta, \nu, \tilde{\eta}, \tilde{\nu}$ are non-negative constants satisfying the constraints $\beta>\rho(\eta+\nu)$ and $\theta>0$.

Market orders are self-exciting because their arrival rates $\lambda^{ \pm}$jump upon the arrival of influential orders (note that the arrival of non-influential orders do not affect $\lambda^{ \pm}$). If the influential market order was a buy (so that a sell limit order was lifted), the jump activity on the buy side increases by $\eta$ while the jump activity on the sell side increases by $\nu$. On the other hand, if the influential market order was a sell (so that a buy limit order was hit), the jump activity on the sell side increases by $\eta$ while the jump activity on the buy side increases by $\nu$. Typically one would expect $\nu<\eta$ so that jumps on the opposite side of the LOB are smaller than jumps on the same side (this bears out in the statistical calibration as well as in the moving window activities reported in Figure 3.1).

News also affects market activity, but does not cause self-excitations. In our model we include cross effects to capture the fact that market participants do not always interpret news in the same way, for example good news increases the intensity of buy market orders by $\tilde{\nu}$ but also affects the intensity of market sell orders by $\tilde{\eta}$.

Trading intensity is mean reverting. Jumps in activity decay back to its long run level of $\theta$ at an exponential rate $\beta$. Figure 3.2 illustrates a sample path during which no news arrives, but some of the market orders that arrive are influential and induce jumps in the activity level. The lower bound condition on $\beta$ is required for the activity rate to be

\footnotetext{
${ }^{25}$ There are automated news feeds designed for AT that already classify the news as good, bad, and neutral.
} 


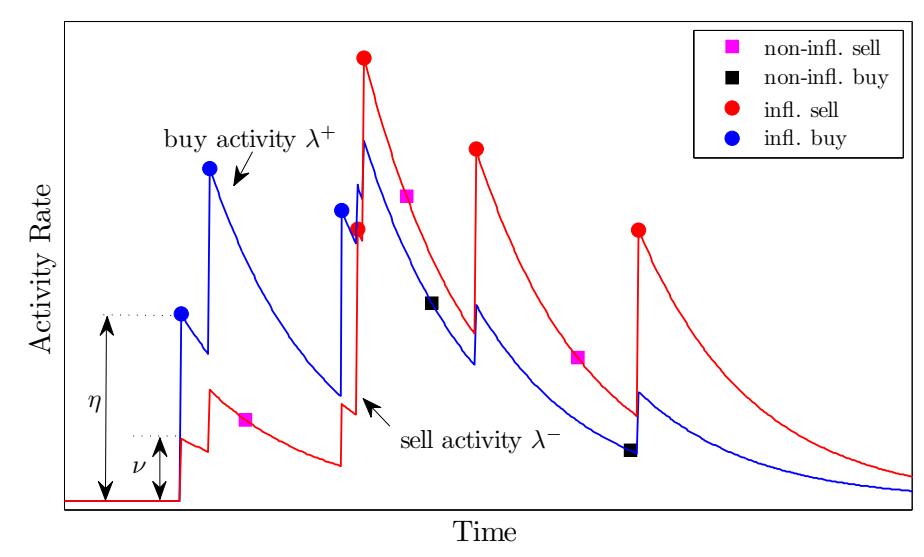

Figure 3.2: Sample path of market order activity rates. When influential trades arrive, the activity of both buy and sell orders increase but by differing amounts. Circles indicate the arrival of an influential market order, while squares indicate the arrival of non-influential trades.

ergodic. To see this, define the mean future activity rate $m_{t}^{ \pm}(u)=\mathbb{E}\left[\lambda_{u}^{ \pm} \mid \mathcal{F}_{t}\right]$ for $u \geq t$. Note that $\lambda_{t}^{ \pm}$is measurable with respect to $\mathcal{F}_{t}$. For the processes $\lambda_{t}^{ \pm}$to be ergodic, $m_{t}^{ \pm}(u)$ must remain bounded as a function of $u$, for each $t$, and the following lemma provides a justification for the constraint.

Lemma 3.2.2. Lower Bound on Mean-Reversion Rate. The mean future rate $m_{t}^{ \pm}(u)$ remains bounded for all $u \geq t$ if and only if $\beta>\rho(\eta+\nu)$. Furthermore,

$$
\lim _{u \rightarrow \infty} m_{t}^{ \pm}(u)=\mathbf{A}^{-1} \boldsymbol{\zeta}, \text { where } \mathbf{A}=\left(\begin{array}{cc}
\beta-\eta \rho & -\nu \rho \\
-\nu \rho & \beta-\eta \rho
\end{array}\right) \quad \text { and } \boldsymbol{\zeta}=\left(\begin{array}{c}
\beta \theta+\tilde{\eta} \mu^{-}+\tilde{\nu} \mu^{+} \\
\beta \theta+\tilde{\nu} \mu^{-}+\tilde{\eta} \mu^{+}
\end{array}\right)
$$

Proof. See Appendix C.1.

The intuition for the constraint is that when a market order arrives the activity will jump either by $\eta$ or by $\nu$ and this occurs with probability $\rho$. Further, since both sell and buy influential orders induce self-excitations, the decay rate $\beta$ must be strong enough to compensate for both jumps to pull the process toward its long-run level of $\theta$.

\subsection{Limit Quote Arrival Dynamics and Fill Rates}

The LOB can take on a variety of shapes, and it changes dynamically throughout the day, see Roşu (2009) and Cont et al. (2010). Market orders eat into the LOB until all 
the volume specified in the order is filled. Limit orders in the tails of the LOB are far less likely to be filled than those within a couple of ticks from the midprice $S_{t}$. Another important feature of the LOB dynamics is how quickly the book recovers from a large market order; i.e. the quoted spread returns to previous values. This is known as the resilience of the $\mathrm{LOB}$.

Therefore, the decision on where to post limit buy and sell orders depends on a number of characteristics of the LOB and on market orders. Some of the relevant features are shape of the LOB, resiliency of the LOB, and how the LOB changes in between the arrival of market orders. These features, combined with the size and rate of the incoming market orders, determine the fill rates of the limit orders. The fill rate is the rate of execution of a limit order. Intuitively, a high (low) fill rate indicates that a limit order is more (less) likely to be filled by a market order.

Here we model the fill rate facing the HFT in a general framework where we allow the depth and shape of the book to fluctuate. The fill rate depends on where the HFT posts the limit buy and sell orders, that is at $S_{t}-\delta_{t}^{-}$and $S_{t}+\delta_{t}^{+}$respectively, where $\delta_{t}^{ \pm}$ denotes how far from the midprice the orders are posted at time $t$. Note that the agent continuously adjusts their posting relative to the midprice, hence, it is not possible for the midprice to move through the agent's posts. Rather, fills occur when MOs arrive and reach the level at which the agent is posted. This is in line with how a number of other authors have modeled optimal postings and fill probabilities (FPs); see Ho and Stoll (1981), Avellaneda and Stoikov (2008), Bayraktar and Ludkovski (2012), Cartea and Jaimungal (2013b), and Guéant et al. (2013). This approach can be viewed as a reduced form one, in contrast to models which focus on modeling the dynamics of each level of the LOB, together with MO arrivals (see, e.g., Roşu (2009) and Cont et al. (2010)). In our reduced form approach, in contrast to previous works, we allow the fill probability itself to be stochastic and react to MOs.

Assumption 3.3.1. The fill rates are of the form $\Lambda_{t}^{ \pm} \triangleq \lambda_{t}^{ \pm} h_{ \pm}\left(\delta ; \boldsymbol{\kappa}_{t}\right)$, where the nonincreasing function $h_{ \pm}\left(\delta ; \boldsymbol{\kappa}_{t}\right): \mathbb{R} \rightarrow[0,1]$ is $C^{2}$ in $\delta$ (uniformly in $t$ for $\boldsymbol{\kappa}_{t} \in \mathbb{R}^{n}$, fixed $\omega \in \Omega)$, and $\lim _{\delta \rightarrow \infty} \delta h_{ \pm}\left(\delta ; \boldsymbol{\kappa}_{t}\right)=0$ for every $\boldsymbol{\kappa}_{t} \in \mathbb{R}^{n}$. Moreover, the functions $h_{ \pm}\left(\delta ; \boldsymbol{\kappa}_{t}\right)$ satisfy: $h_{ \pm}\left(\delta ; \boldsymbol{\kappa}_{t}\right)=1$ for $\delta \leq 0, \boldsymbol{\kappa}_{t} \in \mathbb{R}^{n}$.

Assumption 3.3.1 allows for very general dynamics on the LOB through the dependence of the FPs $h_{ \pm}\left(\delta ; \boldsymbol{\kappa}_{t}\right)$ on the process $\left\{\boldsymbol{\kappa}_{t}\right\}_{0 \leq t \leq T}$. The FPs can be viewed as a parametric collection, with the exponential class $h_{ \pm}\left(\delta ; \boldsymbol{\kappa}_{t}\right)=e^{-\kappa_{t}^{ \pm} \delta}$ and power law class $h_{ \pm}\left(\delta ; \boldsymbol{\kappa}_{t}\right)=\left(1+\left(\kappa_{t}^{ \pm} \delta\right)^{\gamma^{ \pm}}\right)^{-1}$ being two prime examples where $\boldsymbol{\kappa}_{t}=\left(\kappa_{t}^{-}, \kappa_{t}^{+}\right)$. The process $\boldsymbol{\kappa}_{t}$ introduces dynamics into the collection of FPs reflecting the dynamics in the LOB itself. The differentiability requirements in Assumption 3.3.1 are necessary for the asymptotic expansions we carry out later on to be correct. The limiting behavior for large 
$\delta$ implies that the book (volume) thins out sufficiently slowly such that the FPs decay sufficiently fast (faster than linear) so that it is never optimal to place orders infinitely far away from the midprice. Finally, the requirement that $h_{ \pm}\left(\delta ; \boldsymbol{\kappa}_{t}\right)=1$ for $\delta \leq 0$ and $\forall \boldsymbol{\kappa}_{t} \in \mathbb{R}^{n}$ is that of a financial one. A trader wanting to maximize her chances of being filled the next time a market order arrives must post the limit orders at the midprice, i.e., $\delta=0$, or she can also cross the midprice, i.e., $\delta<0$. In these cases we suppose that the fill rate is $\Lambda_{t}^{ \pm}=\lambda_{t}^{ \pm}$; i.e., it equals the rate of incoming market orders. This assumption makes crossing the midprice a suboptimal decision because the trader cannot improve the arrival rate of market orders ( since $\Lambda_{t}^{ \pm}$is constant when $\delta^{ \pm} \leq 0$ ) while receiving less spread for posting at a more competitive price. Thus, she will always post limit orders that are $\delta^{ \pm} \geq 0$ away from the midprice. Additionally, this condition is more desirable than explicitly restricting the controls $\delta^{ \pm}$to be non-negative, since it is not necessary to check the boundary condition at $\delta^{ \pm}=0$; it will automatically be satisfied. Moreover, we have the added bonus that the optimal control satisfies the first-order condition.

Assumption 3.3.2. The dynamics for $\boldsymbol{\kappa}_{t}=\left(\kappa_{t}^{-}, \kappa_{t}^{+}\right)$satisfies

$$
\left\{\begin{array}{l}
d \kappa_{t}^{-}=\beta_{\kappa}\left(\theta_{\kappa}-\kappa_{t}^{-}\right) d t+\eta_{\kappa} d \bar{M}_{t}^{-}+\nu_{\kappa} d \bar{M}_{t}^{+}+\tilde{\eta}_{\kappa} d Z_{t}^{-}+\tilde{\nu}_{\kappa} d Z_{t}^{+} \\
d \kappa_{t}^{+}=\beta_{\kappa}\left(\theta_{\kappa}-\kappa_{t}^{+}\right) d t+\nu_{\kappa} d \bar{M}_{t}^{-}+\eta_{\kappa} d \bar{M}_{t}^{+}+\tilde{\nu}_{\kappa} d Z_{t}^{-}+\tilde{\eta}_{\kappa} d Z_{t}^{+}
\end{array}\right.
$$

where $\eta_{\kappa}, \nu_{\kappa}, \tilde{\eta}_{\kappa}$ and $\tilde{\nu}_{\kappa}$ are non-negative constants and $\theta_{\kappa}$ and $\beta_{\kappa}$ are strictly positive constants.

Assumption 3.3.2 is a specific modeling assumption on $\boldsymbol{\kappa}_{t}$ which allows for incoming influential market orders and news events to have an impact on the FPs. An increase (decrease) in the fill rate can be due to two main factors: (i) a decrease (increase) in LOB depth or (ii) an increase (decrease) in the distribution of market order volumes (in a stochastic dominance sense). This is a one-way effect because influential market orders cause jumps in the $\boldsymbol{\kappa}_{t}$ process, but jumps in the FP do not induce jumps in market order arrivals. While it is possible to allow such feedback, empirical investigations (such as those in Large (2007)) demonstrate that the incoming market orders influence the state of the LOB and not the other way around. ${ }^{26}$ The mean-reversion term draws $\kappa_{t}^{ \pm}$to the long-run mean of $\theta_{\kappa}$ so that the impact of influential orders on the LOB is only temporary. Typically, we expect the rate of mean-reversion $\beta_{\kappa}$ for the LOB to be slower than the rate of mean-reversion $\beta$ of the market order activity. In other words, the impact of influential orders persists in the LOB on a longer time scale compared to their effect on market order activity.

Observe that if market order volumes are independent and identically distributed (iid),

\footnotetext{
${ }^{26}$ This is in contrast to Cebirog̃lu and Horst (2013) where the authors pose a model to assess the market impact of limit order submissions.
} 
then the $\kappa_{t}^{ \pm}$processes can be interpreted as parameters directly dictating the shape of the LOB. In particular, if the market order volumes are iid and exponentially distributed and the shape of the LOB is flat, then the probability that a limit order at price level $S_{t} \pm \delta_{t}^{ \pm}$is executed (given that a market order arrives) is equal to $e^{-\kappa_{t}^{ \pm} \delta_{t}^{ \pm}}$. Consequently, $\kappa_{t}^{ \pm}$can be interpreted as the exponential decay factor for the fill rate of orders placed away from the midprice. In order to satisfy the $C^{1}$ condition at $\delta^{ \pm}=0$ and the condition that $h_{ \pm}\left(\delta, \boldsymbol{\kappa}_{t}\right)=1$ for $\delta^{ \pm} \leq 0$, it is necessary to smooth the exponential function at $\delta=0$. This is always possible, though, since there exist $C^{2}$ functions for which the $L^{2}$ distance to the target function is less than any positive constant.

Moreover, immediately after an influential market buy/sell order arrives (which eats into the sell/buy side of the book), the probability (given that a market order arrives) that a limit order at price level $S_{t} \pm \delta_{t}^{ \pm}$is executed is, for the same $\delta^{ \pm}$, smaller than the probability of it being filled before the influential order arrives. The intuition is the following: Immediately after an influential market order arrives, market participants react in anticipation of the increase of market activity they will face and decide to send limit orders to the book. Since many market participants react in similar way, the probability of limit orders being filled, conditional on a market order arriving, decreases. ${ }^{27}$

Although this specific setup (exponential fill probability) creates a microstructure model with zero spread (best bid price $=$ best offer price), our framework easily allows for a non-zero spread. If $h_{ \pm}\left(\delta ; \boldsymbol{\kappa}_{t}\right)=1$ for $\delta$ smaller than some $d>0$, then the spread would be $2 d$. This is because when $h_{ \pm}\left(\delta ; \boldsymbol{\kappa}_{t}\right)=1$, it means that the trader is guaranteed to be filled when the next market order comes in. In theory, this implies that the volume on each side of the book at depth less than $d$ is less than the smallest possible value for the volume of an incoming market order. For continuous distributions on $\mathbb{R}^{+}$for $\mathrm{MO}$ volumes (with non-zero probability in an open neighbourhood of 0 ), this implies that the LOB is empty at depth less than $d$, which can be confirmed in practice.

\footnotetext{
${ }^{27}$ It is also possible to have markets where, conditional on the arrival of a market order, the probability of a limit order being filled increases immediately after the arrival of an influential order. We can incorporate this feature into our model. Note also that in our general framework, immediately after the influential buy/sell market order arrives, the intensities $\lambda^{ \pm}$increase and the overall effect of an influential order on the fill rates $\Lambda^{ \pm}=\lambda_{t}^{ \pm} h_{ \pm}\left(\delta, \boldsymbol{\kappa}_{t}\right)$ is ambiguous when $\lambda_{t}^{ \pm}$and $h_{ \pm}\left(\delta, \boldsymbol{\kappa}_{t}\right)$ move in opposite directions after the arrival of an influential order - for example, when $h_{ \pm}\left(\delta, \boldsymbol{\kappa}_{t}\right)=e^{-\kappa_{t}^{ \pm} \delta}$.
} 


\subsection{Short Term Alpha Dynamics: Directional Strate- gies and Adverse Selection}

The actions of market participants affect the dynamics of the midprice via activity in the LOB and/or the execution of market buy and sell orders. For instance, the arrival of public information is impounded in the midprice of the asset as a result of new market orders and the arrival and cancellation of limit orders. Similarly, bursts of activity in buy and/or sell market orders, which are not necessarily the result of the arrival of public information, have market impact by producing momentum in the midprice.

As discussed above, a great deal of the strategies that HFTs employ are directional strategies that take advantage of short-term price deviations. HFTs use their superior speed to gather and process order flow information, as well as public information, to spot fleeting opportunities which, in our model, are captured by the dynamics of short-termalpha. Short-term predictability is a key source of HF trading revenues for two reasons. First, the strategies enable HFTs to exploit their superior knowledge of short-term trends in prices to execute profitable roundtrip trades, and second, they provide key information to update or cancel quotes that can be adversely picked off by other traders. This can be modeled by simply taking $\eta_{\kappa}<\nu_{\kappa}$, which will induce more arrivals of limit buy (sell) quotes when an influential market buy (sell) order arrives.

One can specify the dynamics of the predictable drift $\alpha_{t}$ in many ways and this depends on the factors that affect the short-term drift which for HF market making are based on order flow and news. Here we assume that $\alpha_{t}$ is a zero-mean-reverting process and jumps by a random amount at the arrival times of influential trades and news events. If the influential trade was buy initiated (and therefore lifts a sell limit order) the drift will jump up, and if the influential trade was sell initiated (and therefore hit a buy limit order) the drift will jump down; news has a similar effect on $\alpha_{t}$. As such, we model the predictable drift according to the following assumption.

Assumption 3.4.1. The dynamics for the predictable component of the midprice's drift $\left\{\alpha_{t}\right\}_{0 \leq t \leq T}$ satisfies

$$
d \alpha_{t}=-\zeta \alpha_{t} d t+\sigma_{\alpha} d B_{t}+\epsilon_{M_{t}^{+}}^{+} d \bar{M}_{t}^{+}-\epsilon_{M_{t}^{-}}^{-} d \bar{M}_{t}^{-}+\widetilde{\epsilon}_{Z_{t}^{+}}^{+} d Z_{t}^{+}-\widetilde{\epsilon}_{Z_{t}^{-}}^{-} d Z_{t}^{-}
$$

where $\epsilon_{k}^{ \pm}$and $\widetilde{\epsilon}_{k}^{ \pm}$are iid random variables (independent of all other processes) representing the size of the sell/buy influential trade and news impact on the drift of the midprice. From this point forward, we will omit the subscript for compactness. Moreover, $\left\{B_{t}\right\}_{0 \leq t \leq T}$ denotes a Brownian motion independent of all other processes, and $\zeta, \sigma_{\alpha}$ are positive constants. 
Moreover, we see how slower traders will be adversely selected by better informed and quicker traders. For example, assume that $\alpha_{t}=0$ and an HFT "detects" that the incoming buy market order is influential. Her optimal directional strategy is to simultaneously send the following orders to the LOB: cancel her sell limit orders, attempt purchase the asset (from a slower market participant), and send new sell limit orders to be able to unwind the transaction. Of course, these types of trades do not guarantee a profit, but on average these roundtrips will be profitable because the HFT trades on short-term-alpha and profits from other traders who are not able to update their quotes in time or who submit market sell orders right before prices increase. Finally, even if HFTs who are able to trade on short-term-alpha miss a fleeting opportunity to execute a directional trade, they still benefit from updating their stale quotes in the LOB to avoid being adversely selected by other market participants. Given our chosen dynamics on the fill probability driving process $\kappa_{t}^{ \pm}$in (3.3), the aforementioned effect can be modeled by taking $\eta_{\kappa}<\nu_{\kappa}$, which will induce more arrivals of limit buy (sell) quotes when an influential market buy (sell) order arrives.

An alternative approach to adverse selection was introduced in Cartea and Jaimungal (2013b), whereby MOs may induce an immediate jump in the midprice. The result of such direct adverse selection effects was that the agent increases her optimal postings by the expected jump size. In this chapter, we will see a similar, but distinct, result whereby the agent adjusts her posting to protect herself against the potential change in the midprice drift.

\subsection{The HFT's Optimization Problem}

Thus far, we have specified counting processes for market orders and dynamics of the LOB through the FPs; however, we also require a counting process for the agent's filled limit orders. To this end, let the processes $\left\{N_{t}^{+}\right\}_{0 \leq t \leq T}$ and $\left\{N_{t}^{-}\right\}_{0 \leq t \leq T}$ denote the number of the agent's limit sell and buy orders, respectively, that were filled up to and including time $t$, and the process $q_{t}=N_{t}^{-}-N_{t}^{+}$is the agent's total inventory. Note that the arrival rate of these counting processes can be expressed as $\Lambda_{t}^{ \pm}=\lambda_{t}^{ \pm} h_{ \pm}\left(\delta ; \boldsymbol{\kappa}_{t}\right)$, as in Assumption 3.3.1. Finally, the agent's cash process (i.e. excluding the value of the $q_{t}$ shares she currently holds) satisfies the SDE

$$
d X_{t}=\left(S_{t}+\delta_{t-}^{+}\right) d N_{t}^{+}-\left(S_{t}-\delta_{t-}^{-}\right) d N_{t}^{-}
$$

where $\delta_{t-}^{ \pm}$denotes the left-limit of the LO's distance from the midprice (i.e. if the LO was filled, the agent receives the quote that was posted an instant prior to the arrival of 
the MO). ${ }^{28}$ Note that it is equivalent to use $S_{t}$ instead of $S_{t-}$ in Equation (3.5) since the trajectories of $S_{t}$ are continuous.

\subsubsection{Formulation of the HF Trading Problem}

The HFT wishes to place sell/buy limit orders at the prices $S_{t} \pm \delta_{t}^{ \pm}$at time $t$ such that the expected terminal wealth is maximized while penalizing inventories. ${ }^{29}$ The HFT is continuously repositioning her limit orders in the book by cancelling stale limit orders and submitting new limit orders. ${ }^{30}$ Specifically, her value function is

$$
\Phi(t, x, s, q, \alpha, \boldsymbol{\lambda}, \boldsymbol{\kappa})=\sup _{\left(\delta_{u}^{-}, \delta_{u}^{+}\right)_{t \leq u \leq T} \in \mathcal{A}} \mathbb{E}_{(t, x, s, q, \alpha, \boldsymbol{\lambda}, \boldsymbol{\kappa})}\left[X_{T}+q_{T} S_{T}-\phi \int_{t}^{T} q_{s}^{2} d s\right]
$$

where the supremum is taken over all (bounded) $\mathcal{F}_{t}$ previsible functions and $\phi \geq 0$ penalizes deviations of $q_{t}$ from zero along the entire path of the strategy. Moreover, $\overline{\mathcal{F}}_{t}$ is the natural (and completed) filtration generated by the collection of processes $\left\{S_{t}, \epsilon_{M_{t}^{ \pm}}^{ \pm}, M_{t}^{ \pm}=\bar{M}_{t}^{ \pm}+\widetilde{M}_{t}^{ \pm}, N_{t}^{ \pm}, B_{t}\right\}$ and define the expanded filtration $\mathcal{F}_{t}=\overline{\mathcal{F}}_{t} \vee$ $\sigma\left\{\left(\bar{M}_{u}^{ \pm}\right)_{0 \leq u \leq t}\right\}$. Note that $\boldsymbol{\lambda}_{t}$ and $\boldsymbol{\kappa}_{t}$ are progressively measurable with respect to this expanded filtration. Also notice that we use $\epsilon_{M_{t}^{ \pm}}^{ \pm}$instead of $\epsilon_{\bar{M}_{t}^{ \pm}}^{ \pm}$in the definition of $\overline{\mathcal{F}}_{t}$ so that one is not able to discern which trades were influential given this filtration. We will often suppress the dependence on many of the variables in $\Phi(\cdot)$ and recall that we assumed $v=0$ in the dynamics of the midprice. As discussed in Section 2.1, Cartea et al. (2013) show that the running penalty term in (3.6) can be interpreted as arising from the agent's ambiguity aversion with respect to the asset's midprice.

The above control problem can be cast into a discrete-time controlled Markov chain as carried out in Bäuerle and Rieder (2009). Classical results from Bertsekas and Shreve (1978) imply that a dynamic programming principle (DPP) holds and that the value function is the unique viscosity solution (see Appendix B.2) of the Hamilton-Jacobi-

\footnotetext{
${ }^{28}$ The technical issue is as follows: recall that the driving counting processes, and consequently, the spreads, are right continuous with left-limits (RCLL). However, stochastic integrals must have integrands that are left-continuous with right-limits (LCRL) for the integral w.r.t. a martingale integrator to still be a martingale. Replacing $\delta_{t}^{ \pm}$with $\delta_{t-}^{ \pm}$achieves this goal.

${ }^{29} \mathrm{An}$ alternative specification is to assume that the HFT is risk averse so that she maximizes expected utility of terminal wealth. The current approach, however, is more akin to Almgren (2003), where quadratic variation, rather than variance, is penalized, which acts on the entire path of the strategy.

${ }^{30}$ In this setup, the HFT's limit orders are always of the same size. An interesting extension is to also allow the HFT to choose the number of shares in each limit order.
} 
Bellman (HJB) equation

$$
\begin{aligned}
& \left(\partial_{t}+\mathcal{L}\right) \Phi+\alpha \Phi_{s}+\frac{1}{2} \sigma^{2} \Phi_{s s} \\
& \quad+\lambda^{-} \sup _{\delta^{-}}\left\{h_{-}\left(\delta^{-} ; \boldsymbol{\kappa}\right)\left[\mathbb{S}_{q, \lambda}^{-} \Phi\left(t, x-s+\delta^{-}\right)-\Phi\right]+\left(1-h_{-}\left(\delta^{-} ; \boldsymbol{\kappa}\right)\right)\left[\mathbb{S}_{\lambda}^{-} \Phi-\Phi\right]\right\} \\
& \quad+\lambda^{+} \sup _{\delta^{+}}\left\{h_{+}\left(\delta^{+} ; \boldsymbol{\kappa}\right)\left[\mathbb{S}_{q, \lambda}^{+} \Phi\left(t, x+s+\delta^{+}\right)-\Phi\right]+\left(1-h_{+}\left(\delta^{+} ; \boldsymbol{\kappa}\right)\right)\left[\mathbb{S}_{\lambda}^{+} \Phi-\Phi\right]\right\}=\phi q^{2},
\end{aligned}
$$

with boundary condition $\Phi(T, \cdot)=x+q s$, and the integro-differential operator $\mathcal{L}$ is the part of the infinitesimal generator of the processes $\alpha_{t}, \boldsymbol{\lambda}_{t}, \boldsymbol{\kappa}_{t}$, and $Z_{t}^{ \pm}$which do not depend on the controls $\delta_{t}^{ \pm}$. Explicitly,

$$
\begin{aligned}
\mathcal{L}= & \beta\left(\theta-\lambda^{-}\right) \partial_{\lambda^{-}}+\beta\left(\theta-\lambda^{+}\right) \partial_{\lambda^{+}}+\beta_{\kappa}\left(\theta_{\kappa}-\kappa^{-}\right) \partial_{\kappa^{-}}+\beta_{\kappa}\left(\theta_{\kappa}-\kappa^{+}\right) \partial_{\kappa^{+}} \\
& -\zeta \alpha \partial_{\alpha}+\frac{1}{2} \sigma_{\alpha}^{2} \partial_{\alpha \alpha}+\mu^{-}\left(\widetilde{\mathbb{S}}_{\lambda}^{-}-1\right)+\mu^{+}\left(\widetilde{\mathbb{S}}_{\lambda}^{+}-1\right) .
\end{aligned}
$$

Moreover, we have introduced the following shift operators:

$$
\begin{aligned}
\mathbb{S}_{\lambda}^{ \pm} \Phi & =\rho \mathbb{E}\left[\mathcal{S}_{\lambda}^{ \pm} \Phi\right]+(1-\rho) \Phi, \\
\mathbb{S}_{q \lambda}^{ \pm} \Phi & =\rho \mathbb{E}\left[\mathcal{S}_{q \lambda}^{ \pm} \Phi\right]+(1-\rho) \mathcal{S}_{q}^{ \pm} \Phi, \\
\mathcal{S}_{q \lambda}^{ \pm} & =\mathcal{S}_{q}^{ \pm} \mathcal{S}_{\lambda}^{ \pm} \\
\mathcal{S}_{q}^{ \pm} \Phi(t, x, s, q, \alpha, \boldsymbol{\lambda}, \boldsymbol{\kappa}) & =\Phi(t, x, s, q \mp 1, \alpha, \boldsymbol{\lambda}, \boldsymbol{\kappa}), \\
\mathcal{S}_{\lambda}^{+} \Phi(t, x, s, q, \alpha, \boldsymbol{\lambda}, \boldsymbol{\kappa}) & =\Phi\left(t, x, s, q, \alpha+\epsilon^{+}, \boldsymbol{\lambda}+(\nu, \eta)^{\prime}, \boldsymbol{\kappa}+\left(\nu_{\kappa}, \eta_{\kappa}\right)^{\prime}\right), \\
\mathcal{S}_{\lambda}^{-} \Phi(t, x, s, q, \alpha, \boldsymbol{\lambda}, \boldsymbol{\kappa}) & =\Phi\left(t, x, s, q, \alpha-\epsilon^{-}, \boldsymbol{\lambda}+(\eta, \nu)^{\prime}, \boldsymbol{\kappa}+\left(\eta_{\kappa}, \nu_{\kappa}\right)^{\prime}\right), \\
\widetilde{\mathbb{S}}_{\lambda}^{+} \Phi(t, x, s, q, \alpha, \boldsymbol{\lambda}, \boldsymbol{\kappa}) & =\mathbb{E}\left[\Phi\left(t, x, s, q, \alpha+\widetilde{\epsilon}^{+}, \boldsymbol{\lambda}+(\tilde{\nu}, \tilde{\eta})^{\prime}, \boldsymbol{\kappa}+\left(\widetilde{\nu}_{\kappa}, \widetilde{\eta}_{\kappa}\right)^{\prime}\right)\right], \\
\widetilde{\mathbb{S}}_{\lambda}^{-} \Phi(t, x, s, q, \boldsymbol{\lambda}, \boldsymbol{\kappa}) & =\mathbb{E}\left[\Phi\left(t, x, s, q, \alpha-\widetilde{\epsilon}^{-}, \boldsymbol{\lambda}+(\tilde{\eta}, \tilde{\nu})^{\prime}, \boldsymbol{\kappa}+\left(\widetilde{\eta}_{\kappa}, \widetilde{\nu}_{\kappa}\right)^{\prime}\right)\right] .
\end{aligned}
$$

The expectation operator $\mathbb{E}[\cdot]$ in $(3.9 \mathrm{a})$ and $(3.9 \mathrm{~b})$ is over the random variables $\epsilon^{ \pm}$and the expectation operator $\mathbb{E}[\cdot]$ in $(3.9 \mathrm{~g})$ and $(3.9 \mathrm{~h})$ is over the random variables $\widetilde{\epsilon}^{ \pm}$.

The terms of the operator $\mathcal{L}$ have the usual interpretations: the first and second terms cause the activity rates $\lambda^{ \pm}$to decay back to the long run level $\theta$. The third and fourth terms pull $\kappa^{ \pm}$to their long run level. The fifth and sixth term causes $\alpha_{t}$ to diffusive and mean-revert to zero. The seventh and eighth terms cause market order activities to jump upon public news arrival. Furthermore, the various terms in the HJB equation represent the jumps in the activity rate and/or a limit order being filled together with the drift and diffusion of the asset price and the effect of penalizing deviations of inventories from zero along the entire path of the strategy is captured by the term $\phi q^{2}$. More specifically, the supremum over $\delta^{-}$contain the terms due to the arrival of a market sell order (which are filled by limit buy orders). The first term represents the arrival of a market order (influential or not) which fills the limit order, while the second term represents the arrival 
of a market order (influential or not) which does not reach the limit order's price level. The supremum over $\delta^{+}$contain the analogous terms for the market buy orders (which are filled by limit sell orders).

\subsubsection{The Feedback Control of the Optimal Trading Strategy}

In general, an exact optimal control is not analytically tractable; two exceptions are the cases of an exponential and power FPs where the optimal control admits exact analytical solutions, as presented in Appendix D.1. For the general case, we provide an approximate optimal control via an asymptotic expansion which is correct to $o(\varsigma)$ where $\varsigma=\max \left(\phi,|\alpha|, \mathbb{E}\left[\epsilon^{ \pm}\right]\right)$. In principle, the expansion can be carried to higher orders if so desired.

Proposition 3.5.1. Optimal Trading Strategy, Feedback Control Form. The value function $\Phi$ admits the decomposition $\Phi=x+q s+g(t, q, \alpha, \boldsymbol{\lambda}, \boldsymbol{\kappa})$ with $g(T, \cdot)=0$. Furthermore, assume that $g(\cdot)$ can be written as an asymptotic expansion as follows:

$$
g(t, q, \alpha, \boldsymbol{\lambda}, \boldsymbol{\kappa})=g_{0}(t, q, \boldsymbol{\lambda}, \boldsymbol{\kappa})+\alpha g_{\alpha}(t, q, \boldsymbol{\lambda}, \boldsymbol{\kappa})+\varepsilon g_{\varepsilon}(t, q, \boldsymbol{\lambda}, \boldsymbol{\kappa})+\phi g_{\phi}(t, q, \boldsymbol{\lambda}, \boldsymbol{\kappa})+o(\varsigma)
$$

with boundary conditions $g .(T, \cdot)=0$. Note that the subscripts on the functions $g$ do not denote derivatives; rather they are labels, and we have written $\mathbb{E}\left[\epsilon^{ \pm}\right]=\varepsilon \mathfrak{a}^{ \pm}$with $\varepsilon$ constant. Then, the feedback controls of the optimal trading strategy for the HJB equation (3.7) admit the expansion

$$
\delta_{t}^{ \pm *}=\delta_{0}^{ \pm}+\alpha \delta_{\alpha}^{ \pm}+\varepsilon \delta_{\varepsilon}^{ \pm}+\phi \delta_{\phi}^{ \pm}+o(\varsigma)
$$

where

$$
\begin{aligned}
& \delta_{\alpha}^{ \pm}=-B\left(\delta_{0}^{ \pm} ; \boldsymbol{\kappa}\right)\left(\mathbb{S}_{q \lambda}^{ \pm} g_{\alpha}-\mathbb{S}_{\lambda}^{ \pm} g_{\alpha}\right) \\
& \delta_{\varepsilon}^{ \pm}=-B\left(\delta_{0}^{ \pm} ; \boldsymbol{\kappa}\right)\left(\mathbb{S}_{q \lambda}^{ \pm} g_{\varepsilon}-\mathbb{S}_{\lambda}^{ \pm} g_{\varepsilon} \pm \rho \mathfrak{a}^{ \pm}\left(\mathcal{S}_{q \lambda}^{ \pm} g_{\alpha}-\mathcal{S}_{\lambda}^{ \pm} g_{\alpha}\right)\right) \\
& \delta_{\phi}^{ \pm}=-B\left(\delta_{0}^{ \pm} ; \boldsymbol{\kappa}\right)\left(\mathbb{S}_{q \lambda}^{ \pm} g_{\phi}-\mathbb{S}_{\lambda}^{ \pm} g_{\phi}\right)
\end{aligned}
$$

and the coefficient $B\left(\delta_{0}^{ \pm} ; \boldsymbol{\kappa}\right)=\frac{h_{ \pm}^{\prime}\left(\delta_{0}^{ \pm} ; \boldsymbol{\kappa}\right)}{2 h_{ \pm}^{\prime}\left(\delta_{0}^{ \pm} ; \boldsymbol{\kappa}\right)+\delta_{0}^{ \pm} h_{ \pm}^{\prime \prime}\left(\delta_{0}^{ \pm} ; \boldsymbol{\kappa}\right)}$. Moreover, $\delta_{0}^{ \pm}$is a strictly positive solution to

$$
\delta_{0}^{ \pm} h_{ \pm}^{\prime}\left(\delta_{0}^{ \pm} ; \boldsymbol{\kappa}\right)+h_{ \pm}\left(\delta_{0}^{ \pm} ; \boldsymbol{\kappa}\right)=0 .
$$

A solution to (3.13) always exists. Furthermore, the exact optimal controls are nonnegative. 
Proof. See Appendix C.2.

In the next section, we use the optimal controls derived here to solve the nonlinear HJB equation and obtain analytical expressions for $g_{\alpha}, g_{\epsilon}$, and $g_{\phi}$ which is the last step we require to determine $\delta_{t}^{ \pm *}$. Before proceeding, we discuss a number of features of the optimal control $\delta_{t}^{ \pm *}$ given by (3.11). The terms on the right-hand side of equation (3.11) show how the optimal postings are decomposed into different components: riskneutral (first term), adverse selection and directional (second and third), and inventorymanagement (fourth term).

The risk-neutral component, given by $\delta_{0}^{ \pm}$, does not directly depend on the arrival rate of market orders, short-term-alpha, news arrival, or inventories. Instead, it depends on the FPs. To see the intuition behind this result, we note that a risk-neutral HFT, who does not penalize inventories, seeks to maximize the probability of being filled at every instant in time. Therefore, the HFT chooses $\delta^{ \pm}$to maximize the expected spread conditional on a market order hitting or lifting the appropriate side of the book, i.e., maximizes $\delta^{ \pm} h_{ \pm}\left(\delta^{ \pm} ; \boldsymbol{\kappa}_{t}\right)$. The first order condition of this optimization problem is given by (3.13), where we see that $\lambda^{ \pm}$plays no role in how the limit orders are calculated. ${ }^{31}$

The optimal halfspreads are adjusted by the impact that influential orders and news have on short-term-alpha through the term $\alpha_{t} \delta_{\alpha}^{ \pm}+\varepsilon \delta_{\varepsilon}^{ \pm}$to reduce adverse selection costs and to profit from directional strategies. An HFT that is able to process information and estimate the parameters of short-term-alpha will adjust the halfspreads to avoid adverse selection and to profit from short-lived trends in the midprice. For example, if shortterm-alpha is positive, the HFT's sell halfspread is increased to avoid being picked off, and at the same time the buy halfspread decreases to take advantage of the first leg of a directional strategy by increasing the probability of purchasing the asset in anticipation of a price increase.

Finally, the fourth term is an inventory management component that introduces asymmetry in the LO postings so that the HFT does not build large long or short inventories. This component of the halfspread is proportional to the penalization parameter $\phi>0$ which induces mean reversion to the optimal inventory position.

\footnotetext{
${ }^{31}$ If there are multiple solutions to (3.13), the HFT chooses the $\delta^{ \pm}$that yields the maximum of $\delta^{ \pm} h_{ \pm}\left(\delta^{ \pm} ; \boldsymbol{\kappa}_{t}\right)$.
} 


\subsubsection{The Asymptotic Solution of the Optimal Trading Strategy}

Armed with the optimal feedback controls, our remaining task is to solve the resulting non-linear HJB equation to first order in $\varsigma$. The following theorem contains a stochastic characterization of the asymptotic expansion of the value function. This characterization can be computed explicitly in certain cases and then plugged into the feedback control to provide the optimal strategies.

Theorem 3.5.2. Solving The HJB Equation. The solutions for $g_{\alpha}, g_{\varepsilon}$ and $g_{\phi}$ can be written as

$$
\begin{aligned}
g_{\alpha} & =a_{\alpha}(t, \boldsymbol{\lambda}, \boldsymbol{\kappa})+q b_{\alpha}(t), \\
g_{\varepsilon} & =a_{\varepsilon}(t, \boldsymbol{\lambda}, \boldsymbol{\kappa})+q b_{\varepsilon}(t, \boldsymbol{\lambda}), \\
g_{\phi} & =a_{\phi}(t, \boldsymbol{\lambda}, \boldsymbol{\kappa})+q b_{\phi}(t, \boldsymbol{\lambda}, \boldsymbol{\kappa})+q^{2} c_{\phi}(t),
\end{aligned}
$$

where

$$
\begin{aligned}
b_{\alpha}(t) & =\frac{1}{\zeta}\left(1-e^{-\zeta(T-t)}\right), \\
b_{\varepsilon}(t, \boldsymbol{\lambda}) & =\mathbb{E}\left[\int_{t}^{T}\left\{\rho\left(\mathfrak{a}^{+} \lambda_{u}^{+}-\mathfrak{a}^{-} \lambda_{u}^{-}\right)+\left(\widetilde{\mathfrak{a}}^{+} \mu^{+}-\widetilde{\mathfrak{a}}^{-} \mu^{-}\right)\right\} b_{\alpha}(u) d u \mid \boldsymbol{\lambda}_{t}=\boldsymbol{\lambda}\right], \\
b_{\phi}(t, \boldsymbol{\lambda}, \boldsymbol{\kappa}) & =2 \mathbb{E}\left[\int_{t}^{T}\left\{h_{0, u}^{+} \lambda_{u}^{+}-h_{0, u}^{-} \lambda_{u}^{-}\right\}(T-u) d u \mid \boldsymbol{\lambda}_{t}=\boldsymbol{\lambda}, \boldsymbol{\kappa}_{t}=\boldsymbol{\kappa}\right], \quad \text { and } \\
c_{\phi}(t) & =-(T-t) .
\end{aligned}
$$

In the above, $h_{0, u}^{ \pm}=h_{ \pm}\left(\delta_{0, u}^{ \pm} ; \boldsymbol{\kappa}_{u}\right)$, and, as before, we have written $\mathbb{E}\left[\epsilon^{ \pm}\right]=\varepsilon \mathfrak{a}^{ \pm}$and $\mathbb{E}\left[\widetilde{\epsilon}^{ \pm}\right]=\varepsilon \widetilde{\mathfrak{a}}^{ \pm}$. Finally, the functions $g_{0}, a_{\alpha}, a_{\varepsilon}$ and $a_{\phi}$ do not affect the optimal strategy.

Proof. See Appendix C.3.

The asymptotic expansion of the optimal controls now follows as a straightforward corollary to Theorem 3.5.2. Note that the function $b_{\varepsilon}$ can be computed explicitly, as is reported in Appendix D.2. Moreover, under some specific assumptions on the FPs $h_{ \pm}$(e.g., if $h_{ \pm}(\cdot)$ are exponential or power law functions), the function $b_{\phi}$ can also be computed explicitly. Proposition 3.5.4 provides a general class of models (which includes the exponential and power law cases) for which simple closed form results are derived, and the implications for the optimal limiting order postings have a very natural interpretation.

Corollary 3.5.3. Optimal Limit Orders. The asymptotic expansion of the optimal controls to first order in $\varsigma$ is (dependencies on the arguments have been suppressed for 
clarity)

$$
\delta^{ \pm *}=\delta_{0}^{ \pm}+B\left(\delta_{0}^{ \pm} ; \boldsymbol{\kappa}\right)\left\{ \pm \mathbb{S}_{\lambda}^{ \pm}\left(\mathbb{E}_{t}\left[\int_{t}^{T} \alpha_{u} d u\right]\right)+\phi\left( \pm \mathbb{S}_{\lambda}^{ \pm} b_{\phi}+(1 \mp 2 q)(T-t)\right)\right\}
$$

where $\delta_{0}^{ \pm}$satisfies (3.13) and we have $\left|\delta_{o p t}^{ \pm}-\delta^{ \pm *}\right|=o(\varsigma)$. Furthermore, the optimal controls $\max \left\{\delta^{ \pm *}, 0\right\}$ are also of order $o(\varsigma) .{ }^{32}$

Proof. See Appendix C.4.

The expression for the optimal control warrants some discussion which goes beyond the discussion that followed the general result in Proposition 3.5.1. The term $\delta_{0}^{ \pm}$represents the action of a risk-neutral agent who is not aware of or is not able to estimate the impact that influential market orders and news arrival have on the stochastic drift of the midprice (so she sets it to zero). The first term in the braces accounts for the expected change in midprice due to the potential impact of orders (and news) on the midprice's drift as well as the expected change to the arrival of orders. That is, this term plays a dual role in the optimal strategy: it corrects for the adverse selection effect and positions the quotes to execute directional strategies. If the drift is positive, the agent posts further away from the midprice on the sell side (adverse selection correction) and closer to the midprice on the buy side in anticipation of upward price movements (directional strategy). When the drift is negative, the interpretation is similar. The term proportional to $\phi$ contains two terms. The first of these terms accounts for LOB volume imbalance and the asymmetry in the arrival rates of market orders on the sell and buy sides, while the second term induces mean reversion to an optimal inventory level which is not necessarily zero.

Closed form expressions for the function $b_{\phi}$ can only be derived under further assumptions on the FPs $h_{ \pm}(\delta ; \boldsymbol{\kappa})$. As a motivating factor, note that both exponential and power law FPs have the property that $h_{ \pm}\left(\delta_{0}^{ \pm} ; \boldsymbol{\kappa}\right)$ are constants, irrespective of the dynamics on the shape parameter $\kappa^{ \pm}$. This leads us to investigate the larger class of models for which $h_{ \pm}\left(\delta_{0}^{ \pm} ; \boldsymbol{\kappa}\right)$ are constant. Under these assumptions, the following proposition provides an explicit form for the function $b_{\phi}$.

Proposition 3.5.4. Explicit Solution for $b_{\phi}(t, \boldsymbol{\lambda}, \boldsymbol{\kappa})$. If $h_{ \pm}\left(\delta_{0}^{ \pm} ; \boldsymbol{\kappa}\right)=h_{ \pm}$are constants $\mathbb{P}$-a.s., then the function $b_{\phi}(t, \boldsymbol{\lambda}, \boldsymbol{\kappa})$ is independent of $\boldsymbol{\kappa}$ and is explicitly given by

$$
b_{\phi}(t, \boldsymbol{\lambda})=2 \boldsymbol{\xi}^{\prime}\left\{\left(\mathbf{A}^{-1}(T-t)-\mathbf{A}^{-2}\left(\mathbf{I}-e^{-\mathbf{A}(T-t)}\right)\right)\left[\boldsymbol{\lambda}-\mathbf{A}^{-1} \boldsymbol{\zeta}\right]+\frac{1}{2}(T-t)^{2} \mathbf{A}^{-1} \boldsymbol{\zeta}\right\}
$$

\footnotetext{
${ }^{32}$ Note that the exact solution of the optimal control is non-negative as discussed in Assumption 3.3.1, but this is not necessarily the case in the asymptotic solution: thus we write the optimal control as $\max \left\{\delta^{ \pm *}, 0\right\}$.
} 
where $\mathbf{I}$ is the $2 \times 2$ identity matrix and $\boldsymbol{\xi}=\left(-h_{-}, h_{+}\right)^{\prime}$.

Proof. Note that $\mathbb{E}\left[\int_{t}^{T} \lambda_{u}^{ \pm}(T-u) d u \mid \mathcal{F}_{t}\right]=\int_{t}^{T} \mathbb{E}\left[\lambda_{u}^{ \pm} \mid \mathcal{F}_{t}\right](T-u) d u=\int_{t}^{T} m_{t}^{ \pm}(u)(T-$ $u) d u$. Using the form of $m_{t}^{ \pm}(u)$ provided in Equation (C.2) and integrating over $u$ implies

$$
\int_{t}^{T} \boldsymbol{m}_{t}(u)(T-u) d u=\left(\mathbf{A}^{-1}(T-t)-\mathbf{A}^{-2}\left(\mathbf{I}-e^{-\mathbf{A}(T-t)}\right)\right)\left(\boldsymbol{\lambda}_{t}-\mathbf{A}^{-1} \boldsymbol{\zeta}\right)+\mathbf{A}^{-1} \boldsymbol{\zeta} \frac{1}{2}(T-t)^{2} .
$$

This result is valid under the restriction that $\mathbf{A}$ is invertible, which is implied by the arrival rate of market orders in Equation (3.2) and Lemma 3.2.2. Moreover, when $h_{ \pm}\left(\delta_{0}^{ \pm} ; \boldsymbol{\kappa}\right)=h_{ \pm}$we have $b_{\phi}(t, \boldsymbol{\lambda})=2 \int_{t}^{T}\left\{h_{+} \cdot m_{t}^{+}(u)-h_{-} \cdot m_{t}^{-}(u)\right\}(T-u) d u$ and $(3.17)$ follows immediately.

As already mentioned, studying the class of models for which $h_{ \pm}\left(\delta_{0}^{ \pm} ; \boldsymbol{\kappa}\right)=h_{ \pm}$are constant was motivated by the exponential and power-law cases which we formalize in the following two examples.

Example 3.5.5. Exponential Fill Rate. Take $\kappa^{ \pm}=f^{ \pm}(\boldsymbol{\kappa})$, where $f^{ \pm}: \mathbb{R}^{k} \rightarrow \mathbb{R}^{+}$ are continuous functions. If $h_{ \pm}(\delta ; \boldsymbol{\kappa})=e^{-\kappa^{ \pm} \delta}$ for $\delta>0$ and $\mathbb{P}\left[\inf _{t \in[0, T]} \kappa_{t}^{ \pm}>0\right]=1$, then $h_{ \pm}\left(\delta_{0}^{ \pm} ; \boldsymbol{\kappa}\right)=e^{-1}$ is constant and Proposition 3.5.4 applies.

Example 3.5.6. Power Fill Rate. Take $\kappa^{ \pm}=f^{ \pm}(\boldsymbol{\kappa})$, where $f^{ \pm}: \mathbb{R}^{k} \rightarrow \mathbb{R}^{+}$are continuous functions, and $\gamma^{ \pm}>1$ are fixed constants. If $h_{ \pm}(\delta ; \boldsymbol{\kappa})=\left[1+\left(\kappa^{ \pm} \delta\right)^{\gamma^{ \pm}}\right]^{-1}$ for

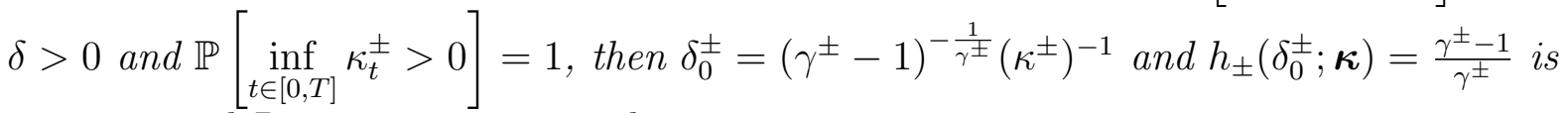
constant and Proposition 3.5.4 applies.

It is worthy to note that the shifted exponential and power law models are also part of this class. Namely $h_{ \pm}(\delta ; \boldsymbol{\kappa})=e^{-\kappa^{ \pm}\left(\delta-d_{ \pm}\right)}$and $h_{ \pm}(\delta ; \boldsymbol{\kappa})=\left[1+\left(\kappa^{ \pm}\left(\delta-d_{ \pm}\right)\right)^{\gamma^{ \pm}}\right]^{-1}$, respectively, for $\delta>d$ and 1 otherwise. In these cases, the asset trades with a spread of $d_{-}+d_{+}$.

Notice that the Poisson model of trade arrivals can be recovered by setting $\rho=0$. In addition, if the initial states $\lambda_{0}^{ \pm}$are equal and $\kappa^{ \pm}$are equal then $b_{\phi} \equiv 0$.

The expression for the optimal control further simplifies considerably when (i) there are no news events (so that $\tilde{\mu}^{+}=\tilde{\mu}^{-}=0$ ); (ii) the impact of influential orders on the stochastic drift is symmetric in the sense that $\varepsilon^{+}=\mathbb{E}\left[\epsilon^{+}\right]=\mathbb{E}\left[\epsilon^{-}\right]=\varepsilon^{-}:=\varepsilon$; (iii) 
the parametric shape of the LOB's FPs are symmetric, in the sense that the class of functions $h^{+}$and $h^{-}$are equal; ${ }^{33}$ and (iv) the fill probability at the risk-neutral optimal control is independent of the scale parameters, ${ }^{34}$ i.e., $h_{ \pm}\left(\delta_{0}^{ \pm}, \boldsymbol{\kappa}\right)=$ const. Under these assumptions, the two important (non-trivial) quantities which appear in the optimal spreads in Equation (3.16) simplify to

$$
\begin{aligned}
\mathbb{E}\left[\int_{t}^{T} \alpha_{u} d u\right] & =\varepsilon \frac{\rho}{\zeta}\left(\lambda_{t}^{+}-\lambda_{t}^{-}\right)\left\{\frac{1-e^{-\widehat{\beta}(T-t)}}{\widehat{\beta}}-\frac{e^{-\zeta(T-t)}-e^{-\widehat{\beta}(T-t)}}{\widehat{\beta}-\zeta}\right\}+\alpha_{t} \frac{1-e^{-\zeta(T-t)}}{\zeta}, \\
b_{\phi} & =2 h\left(\lambda_{t}^{+}-\lambda_{t}^{-}\right)\left\{\frac{1}{\widehat{\beta}}(T-t)-\frac{1-e^{-\widehat{\beta}(T-t)}}{\widehat{\beta}^{2}}\right\},
\end{aligned}
$$

where $\widehat{\beta}=\beta-\rho(\eta-\nu)$ and $h=h_{ \pm}\left(\delta_{0}^{ \pm}, \boldsymbol{\kappa}\right)=$ const. Notice both expressions above contain terms proportional to the difference in the market order activity on the buy and sell sides. If there are no influential orders, these will be equal to their long-run levels and will therefore be zero. However, when influential orders arrive, the buy and sell activities differ, and the agent reacts accordingly. Moreover, the contribution of $\alpha$ accounts for the effect of the mean-reverting stochastic drift of the inventory process.

Corollary 3.5.7. Mean Reversion of Inventory. Given the optimal strategy $\delta^{ \pm}$, the expected drift of the inventory process $q_{t}$, is given by

$$
\lim _{s \rightarrow t^{+}} \frac{1}{s-t} \mathbb{E}\left[q_{s}-q_{t} \mid \mathcal{F}_{t}\right]=\lambda_{t}^{-} h_{-}\left(\delta_{t}^{-} ; \boldsymbol{\kappa}_{t}\right)-\lambda_{t}^{+} h_{+}\left(\delta_{t}^{+} ; \boldsymbol{\kappa}_{t}\right) .
$$

Proof. By observing that $\delta_{t}^{-}, \delta_{t}^{+}, \boldsymbol{\lambda}_{t}$, and $\boldsymbol{\kappa}_{t}$ are all RCLL functions of $t$ (for fixed $\omega \in \Omega$ ), we can conclude that the expected drift in inventories is given by the difference in the arrival rates of filled limit orders at time $t$.

Figure 3.3 illustrates the mean reversion rate of inventory as a function of current inventory $q$ when the FPs are exponential. This function is plotted for three different inventory-aversion levels, as well as for $\alpha=0$ and $\alpha=0.04$. It is clear that when $\alpha=0$ (all else being symmetric), the agent's inventory mean reverts to zero. When $\alpha=0.04$, the agent seeks to acquire inventory to take advantage of the short-term drift of the asset. By symmetry, the corresponding plot of the $\alpha=-0.04$ (with $\lambda_{t}^{-}$and $\kappa_{t}^{-}$replaced with $\lambda_{t}^{+}$and $\kappa_{t}^{+}$, and vice-versa) case will simply be a reflection (about the line $q=0$ ) of Figure 3.3(b).

\footnotetext{
${ }^{33}$ This does not imply that the LOB is symmetric because the scale parameters $\kappa_{t}^{ \pm}$will differ. For example, exponential FPs $e^{-\kappa^{ \pm} \delta^{ \pm}}$satisfy this requirement, even though the book may be significantly deeper on one side than the other.

${ }^{34}$ This condition is satisfied by (but not limited to) the exponential and power law FPs, as discussed
} 


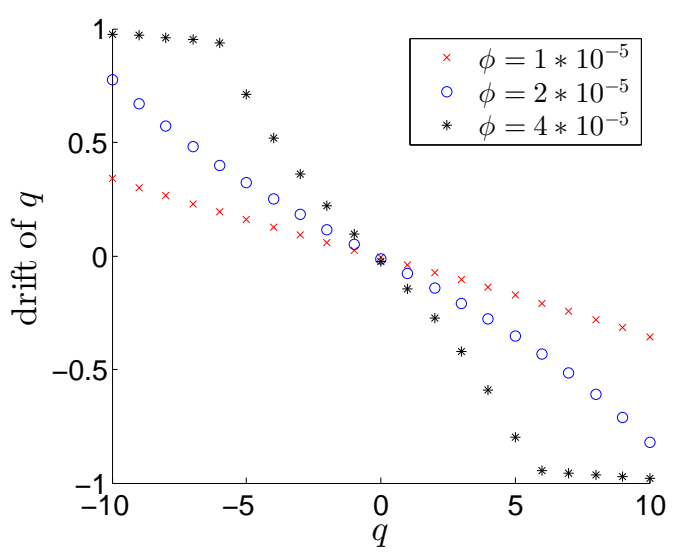

(a) $\alpha=0$

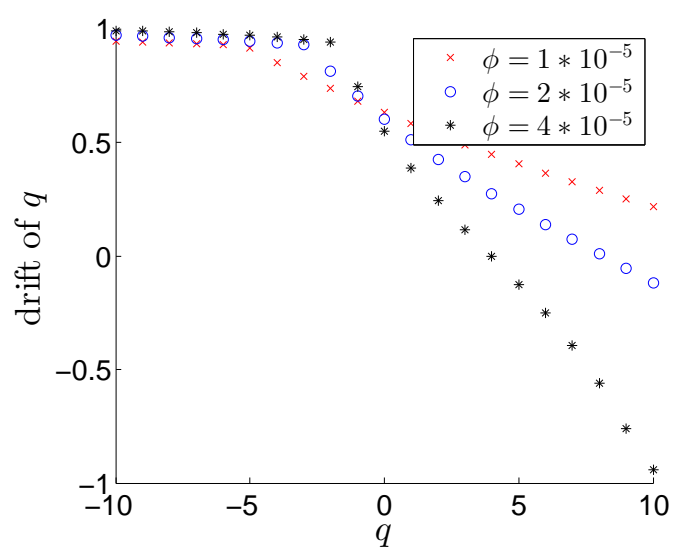

(b) $\alpha=0.04$

Figure 3.3: Mean inventory drift.

\subsection{HF Market Making, Short Term Alpha, and Di- rectional Strategies}

In this section, we apply a simulation study of the HF strategy where buy and sell market orders are generated over a period of 5 minutes. The HFT is rapidly updating her quotes in the LOB by submitting and cancelling limit orders which are filled according to exponential FPs. ${ }^{35}$ The optimal postings are calculated using Corollary 3.5.3 and the explicit form for $b_{\phi}$ in Proposition 3.5.4. The processes $\boldsymbol{\lambda}_{t}, \boldsymbol{\kappa}_{t}$, and $\alpha_{t}$ are updated appropriately, and the terminal cash-flows are stored to produce the profit and loss (PnL) generated from these strategies.

To generate the PnL, we assume that the final inventory is liquidated at the midprice with different proportional transaction costs per share: 1 basis point (bp) and 10 bps. $^{36}$ In practice, the HFT will bear some costs when unwinding a large quantity which could be in the form of a temporary price impact (a consequence of submitting a large market order) and by paying a fee to the exchange for taking liquidity in the form of an aggressive market order. Finally, in each simulation the process is repeated 5, 000 times to obtain the PnLs of the various strategies. More details on the simulation procedure are contained in Appendix E.2.

\footnotetext{
in Examples 3.5.5 and 3.5.6, respectively.

${ }^{35}$ The results for power FPs are very similar, and so in the interest of space we do not show them.

${ }^{36}$ The transaction costs are computed on a percentage basis, and since the starting midprice in the simulations is $\$ 100$, these correspond to approximately 1 and 10 cents per share, respectively. In particular, $q_{T}$ shares are liquidated at a price of $q_{T}\left[S_{T}-c_{\text {trans }} \cdot \operatorname{sign}\left(q_{T}\right)\right]$.
} 
We analyze the performance of the HF market making strategy by varying the quality of the information that the HFT is able to employ when calculating the optimal postings. The main difference between these scenarios is whether the HFT is able to calculate the correct $\rho$ which, conditional on the arrival of an MO, is the probability that the trade is influential and whether they are able to estimate the correct dynamics of shortterm-alpha; all of the HFTs know the equations that determine $\lambda_{t}^{ \pm}$and $\kappa_{t}^{ \pm}$but do not necessarily know the correct parameters. We consider the following eight different HFTs:

1. Correct probability and identification of influential event $(\rho)$. The HFT uses her superior computing power to process information to estimate $\rho$ and the other parameters that determine the dynamics of $\lambda_{t}^{ \pm}$and $\kappa_{t}^{ \pm}$. Furthermore, we assume that the HFT may or may not be able to estimate the correct $\alpha_{t}$ dynamics.

(a) Correct midprice drift $(\alpha)$ dynamics. This is our benchmark because we also assume that the HFT is able to estimate the parameters of the $\alpha_{t}$ process and adjust her postings accordingly.

(b) Zero midprice drift $(\alpha)$ dynamics. Here we assume that although the HFT is able to estimate the correct $\rho$ she assumes that short-term alpha is zero throughout the entire strategy.

2. Filtered influential indicators. Although this HFT has knowledge of the model parameters, she cannot observe which trades are influential and which are not.

(a) Filtered midprice drift $(\alpha)$ dynamics. She employs a particle filter with one particle (as described in Section 5.5.2) to estimate which trades are influential and produce jumps in $\boldsymbol{\lambda}_{t}, \boldsymbol{\kappa}_{t}$, and $\alpha_{t}$ and adjusts her postings accordingly.

(b) Zero midprice drift $(\alpha)$ dynamics. The HFT assumes that short-term-alpha is always zero.

3. High probability of influential event $(\rho)$. At one extreme, we have an HFT who cannot distinguish between the types of market orders and assumes that all orders are influential, $\rho=1$. The jump sizes in $\lambda_{t}^{ \pm}$and $\kappa_{t}^{ \pm}$are set so that the long-run means are $\lambda_{t}^{ \pm}=m_{t}^{ \pm}(\infty)$ and $\kappa_{t}^{ \pm}=\tilde{m}_{t}^{ \pm}(\infty)$.

(a) Incorrect midprice drift $(\alpha)$ dynamics. Because the HFT assumes that all orders are influential she is not able to correctly predict short-term-alpha; she either overestimates or underestimates the effect that market orders have on short-term-alpha because every time there is an incoming market order the HFT will predict a jump in $\alpha_{t}$. The mean jump size parameter, $\varepsilon$, is also rescaled by the correct $\rho$.

(b) Zero midprice drift $(\alpha)$ dynamics. The HFT assumes that short-term-alpha is always zero. 
4. Low probability of influential event $(\rho)$. At the other extreme, we have an HFT who cannot distinguish between order type and assumes that all orders are noninfluential, $\rho=0$, and assumes that $\lambda_{t}^{ \pm}, \kappa_{t}^{ \pm}$are constant and set at their long-run means: $\lambda_{t}^{ \pm}=m_{t}^{ \pm}(\infty)$, given in Lemma 3.2.2, and $\kappa_{t}^{ \pm}=\tilde{m}_{t}^{ \pm}(\infty)$, given in Lemma D.3.1.

(a) Incorrect midprice drift $(\alpha)$ dynamics. Because the HFT assumes that all orders are non-influential she is not able to correctly predict short-term-alpha; she only observes the diffusion components and not the jumps.

(b) Zero midprice drift $(\alpha)$ dynamics. The HFT assumes that short-term-alpha is always zero.

In all eight cases, the data generating process (DGP) is the true process. We assume that news does not arrive during the 5 -minute $(T=300)$ simulation and assume the following values for the parameters (unless otherwise stated): $\beta=60$ and $\theta=1$ (speed and level of mean reversion of intensity of market order arrivals); $\eta=40$ and $\nu=10$ (jumps in $\boldsymbol{\lambda}_{t}$ upon the arrival of influential market orders); $\beta_{\kappa}=10$ and $\theta_{\kappa}=50$ (speed and level of mean reversion for the $\boldsymbol{\kappa}_{t}$ process); $\eta_{\kappa}=10$ and $\nu_{\kappa}=25$ (jumps in $\boldsymbol{\kappa}_{t}$ upon the arrival of influential market orders); $v=0$ (long-term component of the drift of the midprice); $\sigma=0.01$ (volatility of diffusion component of the midprice); $\zeta=2$ and $\sigma_{\alpha}=0.01$ (speed of mean reversion and volatility of diffusion component of $\alpha_{t}$ process); and, finally, we consider the two cases $\rho=0.3$ and $\rho=0.7$ (probability of the market order being influential). Moreover, $\epsilon^{ \pm}$are both exponentially distributed with the same mean, $\mathbb{E}\left[\epsilon^{ \pm}\right]=\varepsilon$, for the buy and sell impacts. In these simulations, we consider two cases: $\varepsilon=0.04$ and $\varepsilon=0.02$.

The first column in Figure 3.4 shows the information that the benchmark HFT employs to calculate her optimal strategy. The top picture shows the dynamics of $\alpha_{t}$ over approximately a two-second window. We see that for this particular time interval of the simulation, $\alpha_{t}$ is negative most of the time and every time an influential order arrives the stochastic drift jumps up (buy market order) or down (sell market order) by a random amount which is drawn from an exponential distribution with mean $\varepsilon=0.04$. In the same column we also show the dynamics of the market order activity $\lambda^{ \pm}$and the FP parameter $\kappa_{t}^{ \pm}$.

In the second column of the same figure we show the information employed by the HFT who incorrectly assumes that all MOs are influential (i.e. assumes $\rho=1$ ) when the true parameter is $\rho=0.7$. In the top picture we observe that by overstating the arrival rate of market orders this HFT also overestimates the impact that MOs have on short-termalpha. The other two pictures in the column show the HFT's estimate of the arrival rate of market orders and fill rates. Obviously, when compared to those used by the benchmark 
HFT these are incorrect estimates of the true $\lambda_{t}^{ \pm}, \kappa_{t}^{ \pm}$because the HFT sets the jump sizes in $\lambda_{t}^{ \pm}$and $\kappa_{t}^{ \pm}$so that the long-run means $m_{t}^{ \pm}(\infty)$ and $\tilde{m}_{t}^{ \pm}(\infty)$ are preserved.

Finally, the last column shows the information used by the HFT who incorrectly assumes that all orders are non-influential $\rho=0$. From the pictures it is clear that this HFT has a poor estimate of $\alpha_{t}, \lambda_{t}^{ \pm}, \kappa_{t}^{ \pm}$.

Figure 3.5 shows how the HFTs post and cancel limit orders during the same two-second window discussed in Figure 3.4 and the inventory-management parameter is $\phi=10^{-5}$. The top row of Figure 3.5, pictures (a), (b), and (c), shows the optimal postings for: the benchmark HFT with correct $\rho=0.7$, the HFT with $\rho=1$, and the HFT with $\rho=0$ respectively, all of which employ the information shown in Figure 3.4. The bottom row of Figure 3.5 shows the postings of the HFTs with the correct and incorrect values of $\rho$, where all of these assume that $\alpha_{t}=0$ and $\varepsilon=0$ along the entire strategy.

To understand the intuition behind the optimal postings of the benchmark HFT, let us focus on Figure 3.5(a). The solid line shows the midprice and the dash-dot lines show the buy and sell limit orders. Circles denote the arrival of influential market orders and squares the arrival of non-influential orders. When the circles and squares are colored in, it shows that the market order was filled by the benchmark HFT. Otherwise, when the circles and squares are not colored in, it represents market orders that arrived but were filled by other more competitive resting orders in the LOB.

One can observe that $\alpha_{t}$ is a key driver of the optimal postings. At the beginning of the window in Figure 3.5(a), we see that an influential sell market order arrived (hitting the buy side of the book), thus short-term-alpha drops (as seen in the top picture in Figure 3.4(a)) and the benchmark HFT cancels her existing limit orders and reposts limit orders that see an increase in the halfspread on the buy side and a decrease in the halfspread on the sell side. These changes in the halfspread are due to adverse selection and directional strategies. Because the benchmark HFT observes a very low $\alpha_{t}$ near the beginning of the window, she knows that negative short-term-alpha over a very short time interval (recall that $\alpha_{t}$ is quickly mean reverting to zero) indicates that if she does not adjust her buy quote downward, she will be picked off by traders that might have anticipated a decline in the midprice (or picked off by a noise trader that sends a sell market order) - thus the benchmark HFT's strategy is to avoid buying the asset right before the price drops. Similarly, the downward adjustment in the sell spread (i.e. adjust the sell quote downward) is part of a directional strategy whereby the benchmark HFT wants to sell the asset before its price drops and then purchase it back at a lower price. ${ }^{37}$

\footnotetext{
${ }^{37}$ The drop in the sell halfspread is also due to inventory management. If the HFT holds a large inventory, she would prefer to sell some of it because of the expected decline in the value of the inventory as a consequence of a drop in the midprice.
} 


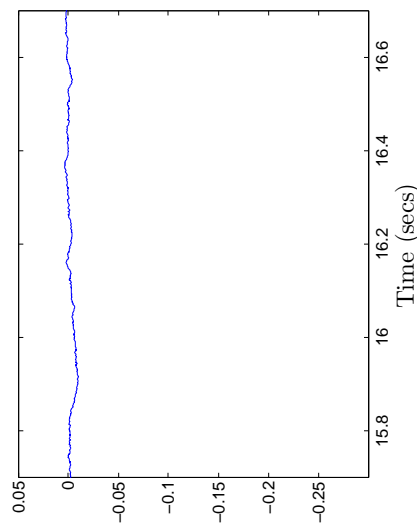

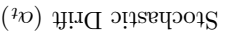

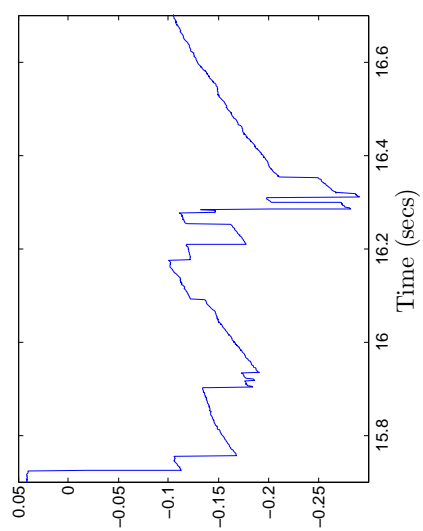

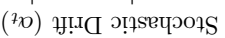

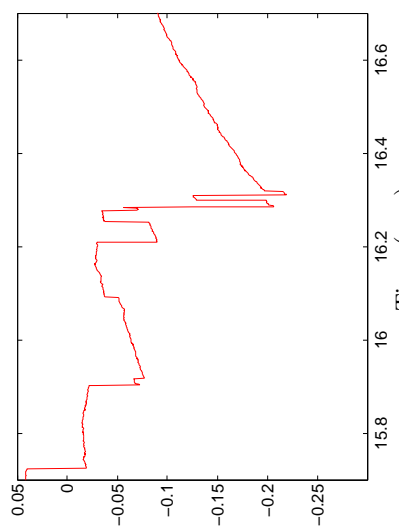

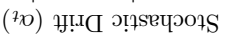

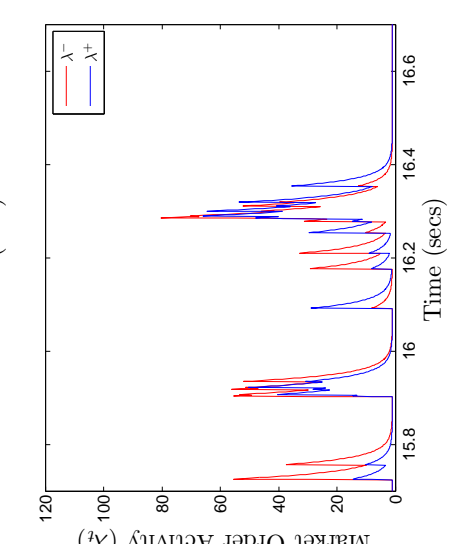

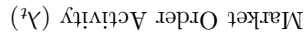

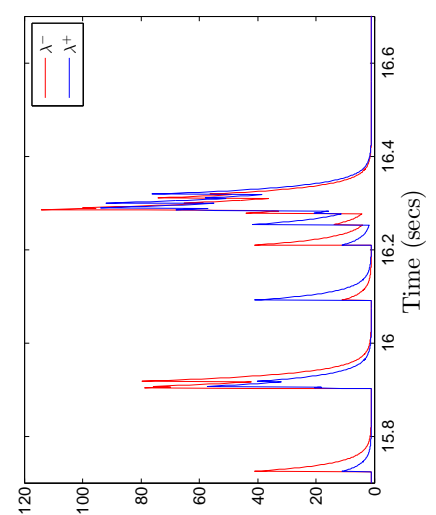

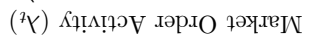

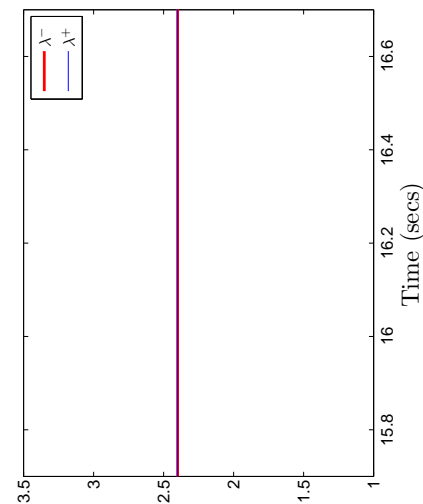

(7Y) Kұ!

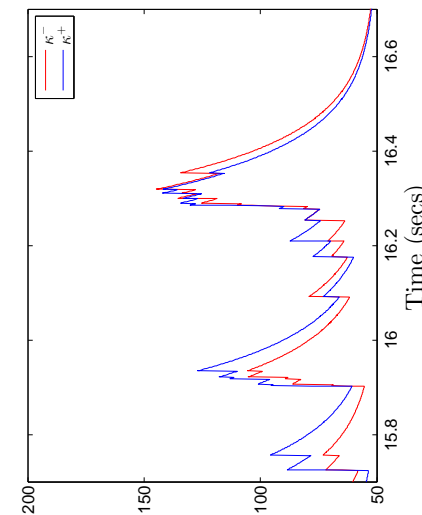

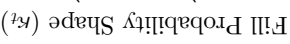

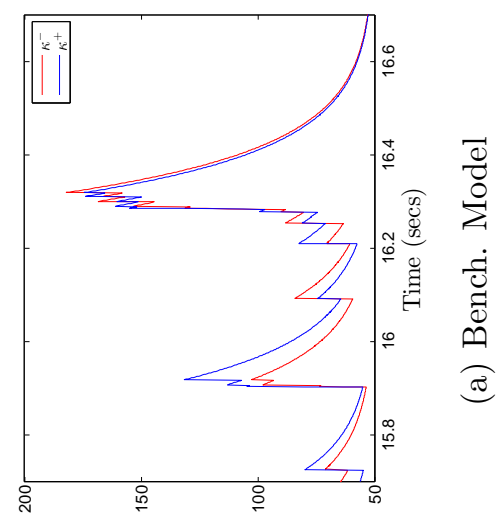

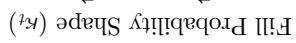

ฮ્త్

禺

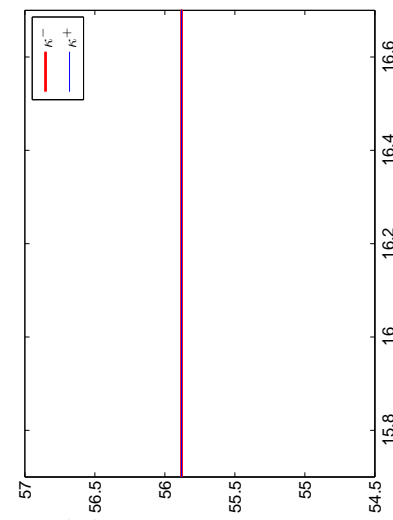

(7y) әdечS Kұ!!!quqo.Id II!H

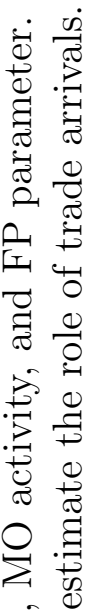



范

过

$+$

幽

$\begin{array}{ll}0 & 0 \\ \Rightarrow & 0\end{array}$

곤

is 0

늘

త్ర્⿹

$\stackrel{\oplus}{\leftrightarrows}$

is 2

$[$ क

I.尹

o

ॠ

車

2.

o

is

$\varangle \stackrel{9}{1}$



0

$\exists$

.00 

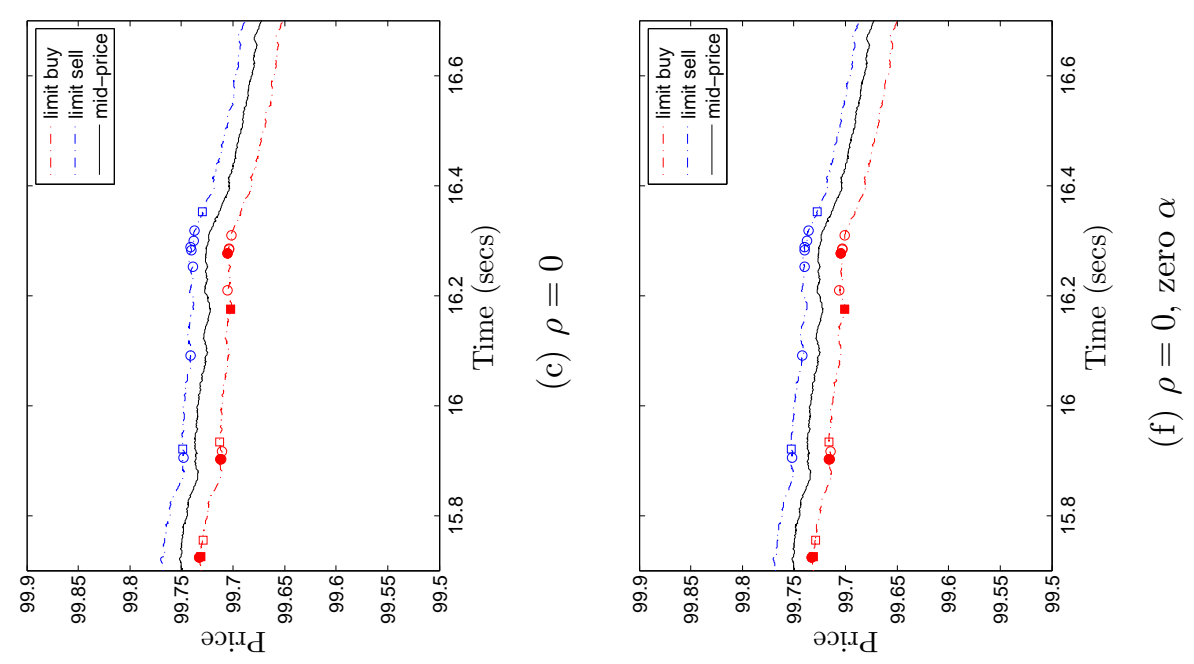

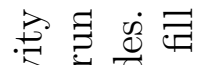

.

음

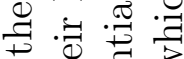

.

范茫

कै

㟧

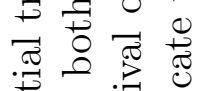

矛

.尹.

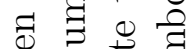

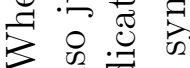
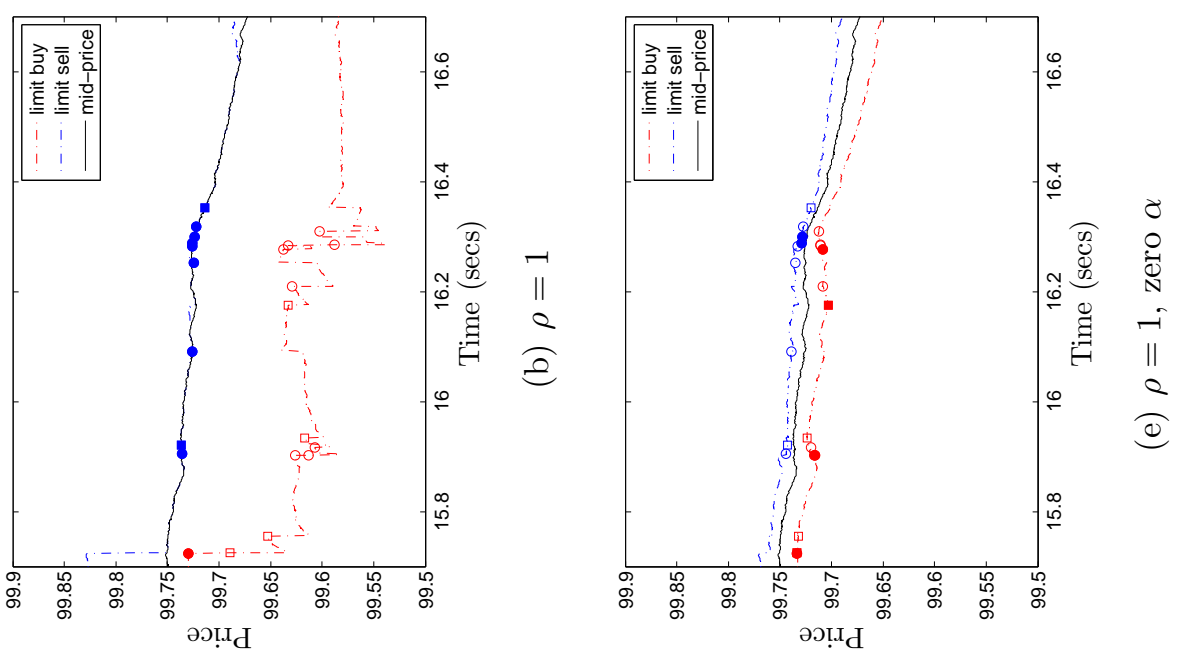

..$\Xi$

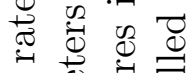

$\Rightarrow \stackrel{0}{\exists}$

표

. సేత్రై

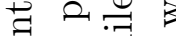

语在 0

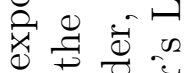

की की

氙苛苛

寻芯

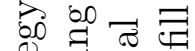

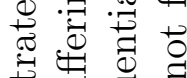

क :
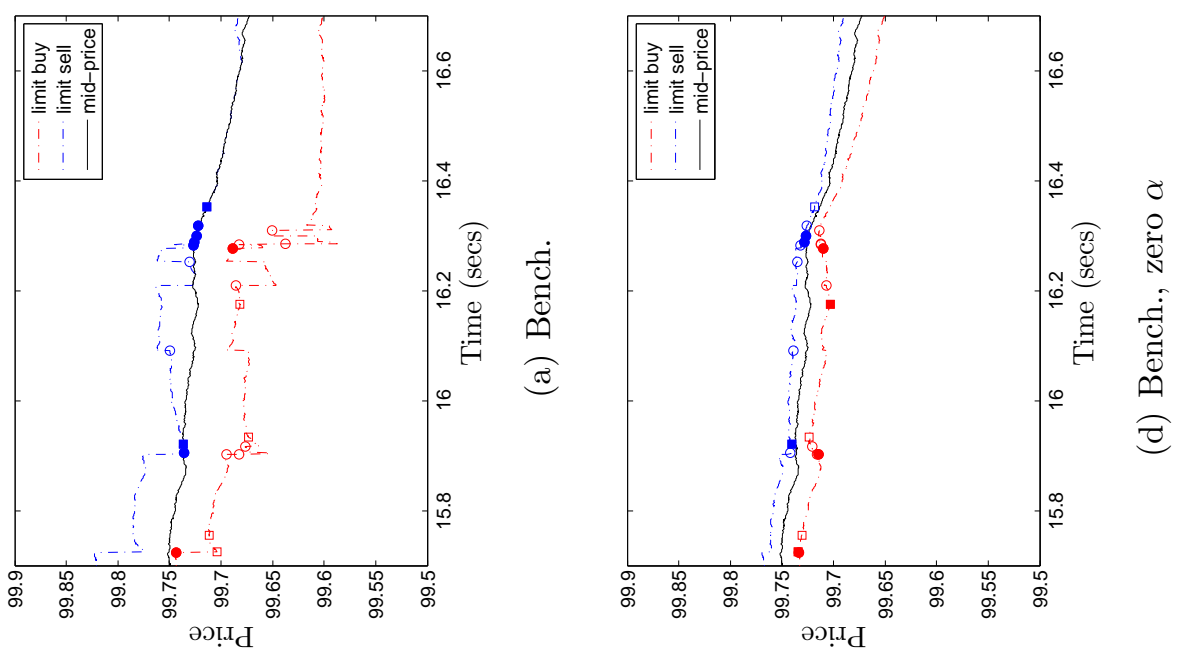

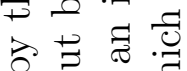

흔

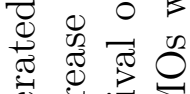

牙记

$\Phi_{\infty 0} . \exists$ च 0

돵 矛要

$\tilde{0}, \stackrel{0}{0}$

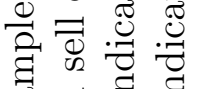

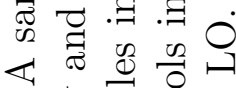

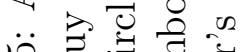

के

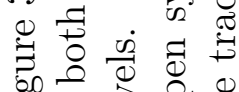

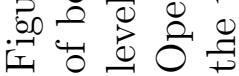


Here we show the PnLs that the HFTs face when executing the optimal strategy. We report the results in Tables 3.1 and 3.2 where the difference between the two tables is the impact that influential orders have $(\varepsilon=0.04$ and $\varepsilon=0.02)$ on short-term-alpha. In both tables, terminal inventories $q_{T}$ are liquidated at the midprice $S_{T}$ and pick up a penalty of $1 \mathrm{bps}$ and $10 \mathrm{bps}$ per share. The tables show the results for different values of the inventory-management parameter $\phi=\left\{1 \times 10^{-5}, 2 \times 10^{-5}, 4 \times 10^{-5}\right\}$. For each value of $\phi$, we show the mean and standard deviation of the eight PnLs where the top row, for each $\phi$, reports the four PnLs resulting from the benchmark HFT (who uses the correct $\rho=0.7$ ), the filtered HFT (who uses advanced the statistical filtering method in Section 5.5.2 when the influential indicators are unobservable), and the other two HFTs who incorrectly specify the arrival of influential and non-influential market orders. For each $\phi$, the bottom row shows the other four PnLs that result from assuming that the HFTs set $\alpha_{t}=0$ throughout the entire strategy.

The tables clearly show that market making is more profitable if the HFTs incorporate in their optimal strategies predictions of short-term-alpha - this is true even if the HFTs incorrectly specify the short-term-alpha parameters. Moreover, when the mean impact of influential orders on $\alpha_{t}$ is $\varepsilon=0.04$, Table 3.1 clearly shows that HFTs who are not able to execute market making strategies based on predictable trends in the midprice will be driven out of the market because their trades are being adversely selected and because they are unable to profit from directional strategies; HFTs who omit short-term-alpha face negative, or at best close to zero, mean PnLs. Table 3.2 shows that if the mean impact of influential orders decreases to $\varepsilon=0.02$, HFTs are able to subsist even if they do not use predictors of short-term-alpha when making markets; however, we believe that in practice HFTs will not survive if they are not able to trade on short-term-alpha to profit from directional strategies and to reduce the effects of adverse selection. ${ }^{38}$

The inventory-management parameter $\phi$ plays an important role in the performance of the HFT strategies. Although the HFTs are maximizing expected terminal wealth (and not expected utility of terminal wealth), they are capital constrained, and their own internal risk-measures require them to penalize building and holding large positions. HFTs that wish to, or are required to, exert a tight control on their exposure to inventories will prefer a high $\phi$. Tables 3.1 and 3.2 show an interesting effect of $\phi$ on the PnL of the different strategies that we study. If the HFT uses her predictions of short-term-alpha to make markets, increasing $\phi$ reduces both the mean and standard deviation of the PnL. Thus, in these cases the tradeoff between mean and standard deviation of profits is clear: those HFTs who trade on short-term-alpha are able to trade off mean against standard deviation of PnL.

\footnotetext{
${ }^{38}$ We are grateful to an anonymous referee of Cartea et al. (2014) for pointing this out.
} 


\begin{tabular}{|c|c|c|c|c|c|c|}
\hline \multicolumn{7}{|c|}{ Case I: $\varepsilon=0.04, \rho=0.7$ and liquidation costs $=1 \mathrm{bp}$} \\
\hline$\phi$ & $\alpha_{t}$ & & Bench. & Filter & $\rho=1$ & $\rho=0$ \\
\hline \multirow{4}{*}{$1 \times 10^{-5}$} & \multirow{2}{*}{ Yes } & mean & 14.09 & 12.07 & 12.77 & -4.34 \\
\hline & & $($ std $)$ & $(6.98)$ & $(6.93)$ & $(6.44)$ & $(3.00)$ \\
\hline & \multirow{2}{*}{ No } & mean & -3.81 & -3.48 & -3.88 & -4.32 \\
\hline & & $(\mathrm{std})$ & $(2.83)$ & $(2.80)$ & $(2.83)$ & $(2.94)$ \\
\hline \multirow{4}{*}{$2 \times 10^{-5}$} & \multirow{2}{*}{ Yes } & mean & 13.52 & 11.66 & 12.15 & -2.80 \\
\hline & & $(\mathrm{std})$ & $(5.44)$ & $(5.40)$ & $(5.01)$ & $(2.21)$ \\
\hline & \multirow{2}{*}{ No } & mean & -1.57 & -1.44 & -1.71 & -2.80 \\
\hline & & (std) & $(2.07)$ & $(2.04)$ & $(2.06)$ & $(2.17)$ \\
\hline \multirow{4}{*}{$4 \times 10^{-5}$} & \multirow{2}{*}{ Yes } & mean & 12.49 & 10.88 & 11.08 & -1.24 \\
\hline & & $(\mathrm{std})$ & $(4.28)$ & $(4.24)$ & $(3.94)$ & $(1.60)$ \\
\hline & \multirow{2}{*}{ No } & mean & 0.24 & 0.27 & 0.06 & -1.25 \\
\hline & & (std) & $(1.48)$ & $(1.46)$ & $(1.48)$ & $(1.58)$ \\
\hline
\end{tabular}

\begin{tabular}{|c|c|c|c|c|c|c|}
\hline \multicolumn{7}{|c|}{ Case II: $\varepsilon=0.04, \rho=0.7$ and liquidation costs $=10 \mathrm{bp}$} \\
\hline$\phi$ & $\alpha_{t}$ & & Bench. & Filter & $\rho=1$ & $\rho=0$ \\
\hline \multirow{4}{*}{$1 \times 10^{-5}$} & \multirow{2}{*}{ Yes } & mean & 13.32 & 11.28 & 12.05 & -4.81 \\
\hline & & $(\mathrm{std})$ & $(6.80)$ & $(6.77)$ & $(6.29)$ & $(3.12)$ \\
\hline & \multirow{2}{*}{ No } & mean & -4.28 & -3.94 & -4.34 & -4.78 \\
\hline & & $(\mathrm{std})$ & $(2.98)$ & $(2.93)$ & $(2.98)$ & $(3.07)$ \\
\hline \multirow{4}{*}{$2 \times 10^{-5}$} & \multirow{2}{*}{ Yes } & mean & 12.87 & 11.00 & 11.55 & -3.19 \\
\hline & & $(\mathrm{std})$ & $(5.30)$ & $(5.29)$ & $(4.90)$ & $(2.32)$ \\
\hline & \multirow{2}{*}{ No } & mean & -1.96 & -1.82 & -2.10 & -3.19 \\
\hline & & $(\mathrm{std})$ & $(2.20)$ & $(2.16)$ & $(2.19)$ & $(2.28)$ \\
\hline \multirow{4}{*}{$4 \times 10^{-5}$} & \multirow{2}{*}{ Yes } & mean & 11.94 & 10.33 & 10.58 & -1.56 \\
\hline & & $(\mathrm{std})$ & $(4.17)$ & $(4.16)$ & $(3.85)$ & $(1.69)$ \\
\hline & \multirow{2}{*}{ No } & mean & -0.09 & -0.05 & -0.27 & -1.57 \\
\hline & & $(\mathrm{std})$ & $(1.59)$ & $(1.57)$ & $(1.59)$ & $(1.67)$ \\
\hline
\end{tabular}

Table 3.1: The mean and standard deviation of the PnL from the various strategies as the inventory-management parameter $\phi$ increases, $\varepsilon=0.04$, and final inventory liquidation costs are 1 bps and 10bps per share. Recall that only the benchmark HFT, who uses $\rho=0.7$, is able to correctly specify the dynamics of short-term-alpha. 


\begin{tabular}{|c|c|c|c|c|c|c|}
\hline \multicolumn{7}{|c|}{ Case III $\varepsilon=0.02, \rho=0.7$ and liquidation costs $=1 \mathrm{bp}$} \\
\hline$\phi$ & & & Bench. & Filter & $\rho=1$ & $\rho=0$ \\
\hline \multirow{4}{*}{$1 \times 10^{-5}$} & \multirow{2}{*}{ Yes } & mean & 8.81 & 7.90 & 8.16 & 1.25 \\
\hline & & $(\mathrm{std})$ & $(2.84)$ & $(2.82)$ & $(2.60)$ & $(1.55)$ \\
\hline & \multirow{2}{*}{ No } & mean & 1.66 & 1.76 & 1.61 & 1.23 \\
\hline & & (std) & $(1.46)$ & $(1.45)$ & $(1.47)$ & $(1.52)$ \\
\hline \multirow{4}{*}{$2 \times 10^{-5}$} & \multirow{2}{*}{ Yes } & mean & 8.29 & 7.47 & 7.63 & 1.84 \\
\hline & & $(\mathrm{std})$ & $(2.23)$ & $(2.19)$ & $(2.04)$ & $(1.15)$ \\
\hline & \multirow{2}{*}{ No } & mean & 2.55 & 2.56 & 2.48 & 1.80 \\
\hline & & $(\operatorname{std})$ & $(1.09)$ & $(1.07)$ & $(1.08)$ & $(1.13)$ \\
\hline \multirow{4}{*}{$4 \times 10^{-5}$} & \multirow{2}{*}{ Yes } & mean & 7.41 & 6.72 & 6.77 & 2.33 \\
\hline & & $(\mathrm{std})$ & $(1.75)$ & $(1.71)$ & $(1.60)$ & $(0.85)$ \\
\hline & \multirow{2}{*}{ No } & mean & 3.08 & 3.04 & 2.99 & 2.30 \\
\hline & & (std) & $(0.81)$ & $(0.80)$ & $(0.80)$ & $(0.84)$ \\
\hline
\end{tabular}

\begin{tabular}{|c|c|c|c|c|c|c|}
\hline \multicolumn{7}{|c|}{ Case IV $\varepsilon=0.02, \rho=0.7$ and liquidation costs $=10 \mathrm{bps}$} \\
\hline$\phi$ & & & Bench. & Filter & $\rho=1$ & $\rho=0$ \\
\hline \multirow{4}{*}{$1 \times 10^{-5}$} & \multirow{2}{*}{ Yes } & mean & 8.16 & 7.25 & 7.57 & 0.79 \\
\hline & & $(\operatorname{std})$ & $(2.75)$ & $(2.74)$ & $(2.53)$ & $(1.69)$ \\
\hline & \multirow{2}{*}{ No } & mean & 1.19 & 1.30 & 1.15 & 0.77 \\
\hline & & $(\operatorname{std})$ & $(1.62)$ & $(1.60)$ & $(1.63)$ & $(1.67)$ \\
\hline \multirow{4}{*}{$2 \times 10^{-5}$} & \multirow{2}{*}{ Yes } & & 7.7 & 6 & 7 & 1.45 \\
\hline & & $(\operatorname{std})$ & $(2.16)$ & $(2.14)$ & $(1.99)$ & $(1.27)$ \\
\hline & \multirow{2}{*}{ No } & mean & 2.16 & 2.18 & 2.09 & 1.42 \\
\hline & & (std) & $(1.23)$ & $(1.20)$ & $(1.22)$ & $(1.26)$ \\
\hline \multirow{4}{*}{$4 \times 10^{-5}$} & \multirow{2}{*}{ Yes } & mean & 6.95 & 6.27 & 6.35 & 2.00 \\
\hline & & $(\operatorname{std})$ & $(1.70)$ & $(1.68)$ & $(1.57)$ & $(0.95)$ \\
\hline & \multirow{2}{*}{ No } & mean & 2.75 & 2.72 & 2.66 & 1.98 \\
\hline & & (std) & $(0.92)$ & $(0.91)$ & $(0.92)$ & $(0.95)$ \\
\hline
\end{tabular}

Table 3.2: The mean and standard deviation of the PnL from the various strategies as the inventory-management parameter $\phi$ increases, $\varepsilon=0.02$, and final inventory liquidation costs are 1 bps and 10bps per share. Recall that only the benchmark HFT, who uses $\rho=0.7$, is able to correctly specify the dynamics of short-term-alpha. 
On the other hand, the effect of increasing $\phi$ on the PnL of HFTs that do not take into account short-term-alpha is to increase the mean and to decrease the standard deviation of the PnL. The intuition behind this result is the following. As we have shown, HFTs that do not trade using predictions of short-term-alpha suffer from being picked off by better informed traders and are unable to boost their profits using directional strategies. However, increasing $\phi$ makes their postings more conservative because, everything else being equal, the limit orders are posted deeper in the LOB, and this makes it more difficult for other traders to pick off their quotes. Thus, by increasing $\phi$, the HFT reduces her exposure to adverse selection and this explains why the mean PnL increases in $\phi$. Finally, the standard deviation of the PnL decreases because, when $\phi$ increases, the strategy induces very quick mean reversion of inventories to zero.

The filtered strategy (the one that is unable to observe which trades are influential) tends to slightly underperform the benchmark strategy, just as one would expect. This is because the filtered strategy is adapted to $\overline{\mathcal{F}}_{t-}$, a filtration that is contained by $\mathcal{F}_{t-}$ which the benchmark strategy is adapted to. Note that this strategy is adapted to the left limit $\overline{\mathcal{F}}_{t-}$ and not $\overline{\mathcal{F}}_{t}$. The details for the construction of estimators for which events are influential are described later in Section 5.5.2.

One surprising result is that, in several cases, the $\rho=1$ case outperforms the filtered strategy, even though the $\rho=1$ strategy is also $\overline{\mathcal{F}}_{t-}$ measurable. This is due to the choice of estimator $\hat{\boldsymbol{\lambda}}_{t}=\frac{1}{M} \sum_{i=1}^{M} \boldsymbol{\lambda}_{t}^{(i)}$ in Section 5.5.2. Although this quantity is unbiased for $\boldsymbol{\lambda}_{t}$, the effect on PnL of under/over-estimating $\boldsymbol{\lambda}_{t}$ is highly asymmetric. If the investor thinks that an MO is influential when it is not, then her risky position is that of a martingale. However, if she thinks an MO is not influential when, in fact, it is, then she risks having her LO filled in a market that is moving against her. It is not clear what estimator $\hat{\boldsymbol{\lambda}}_{t}$ should be used in this context, and this is left for future research.

Finally, we repeat the simulations by assuming that influential orders arrive with probability $\rho=0.3$. Table 3.3 shows the results when we assume that $\varepsilon=0.04$ and $\varepsilon=0.02$ and final inventory liquidation costs are 1bp. Observe that although the $\rho=1$ strategy outperforms the filtered strategy for high $\rho$ (i.e. 0.7), this effect is mitigated when the true $\rho$ is far different from 1 (as in this case when $\rho=0.3$ ). Also note that now the $\rho=0$ agent performs significantly better, relative to the other agents (although still is the worst of the four strategies). The other results are qualitatively the same as those discussed above. We have also run simulations with different parameter choices, and the benchmark HFT always performs better than the other HFTs. 


\begin{tabular}{|c|c|c|c|c|c|c|}
\hline \multicolumn{7}{|c|}{$\varepsilon=0.04, \rho=0.3$ and liquidation costs $=1 \mathrm{bp}$} \\
\hline$\phi$ & & & Bench. & Filter & $\rho=1$ & $\rho=0$ \\
\hline \multirow{4}{*}{$1 \times 10^{-5}$} & \multirow{2}{*}{ Yes } & mean & 4.97 & 4.03 & 4.16 & 2.60 \\
\hline & & $(\mathrm{std})$ & $(1.43)$ & $(1.48)$ & $(1.19)$ & $(1.26)$ \\
\hline & \multirow{2}{*}{ No } & mean & 2.59 & 2.56 & 2.59 & 2.57 \\
\hline & & $($ std) & $(1.21)$ & $(1.22)$ & $(1.22)$ & $(1.23)$ \\
\hline \multirow{4}{*}{$2 \times 10^{-5}$} & \multirow{2}{*}{ Yes } & mean & 4.79 & 3.92 & 4.01 & 2.70 \\
\hline & & $(\mathrm{std})$ & $(1.11)$ & $(1.13)$ & $(0.92)$ & $(0.94)$ \\
\hline & \multirow{2}{*}{ No } & mean & 2.78 & 2.74 & 2.74 & 2.67 \\
\hline & & $($ std) & $(0.91)$ & $(0.91)$ & $(0.91)$ & $(0.92)$ \\
\hline \multirow{4}{*}{$4 \times 10^{-5}$} & \multirow{2}{*}{ Yes } & mean & 4.47 & 3.70 & 3.72 & 2.75 \\
\hline & & $($ std $)$ & $(0.87)$ & $(0.88)$ & $(0.71)$ & $(0.70)$ \\
\hline & \multirow{2}{*}{ No } & mean & 2.88 & 2.83 & 2.81 & 2.72 \\
\hline & & $($ std) & $(0.68)$ & $(0.69)$ & $(0.69)$ & $(0.70)$ \\
\hline \multicolumn{7}{|c|}{$\varepsilon=0.02, \rho=0.3$ and liquidation costs $=1 \mathrm{bp}$} \\
\hline$\phi$ & & & Bench. & Filter & $\rho=1$ & $\rho=0$ \\
\hline \multirow{4}{*}{$1 \times 10^{-5}$} & \multirow{2}{*}{ Yes } & mean & 4.69 & 4.30 & 4.34 & 3.86 \\
\hline & & (std) & $(0.81)$ & $(0.82)$ & $(0.74)$ & $(0.80)$ \\
\hline & \multirow{2}{*}{ No } & mean & 3.87 & 3.84 & 3.85 & 3.83 \\
\hline & & (std) & $(0.77)$ & $(0.77)$ & $(0.77)$ & $(0.78)$ \\
\hline \multirow{4}{*}{$2 \times 10^{-5}$} & \multirow{2}{*}{ Yes } & mean & 4.51 & 4.15 & 4.19 & 3.82 \\
\hline & & (std) & $(0.65)$ & $(0.65)$ & $(0.59)$ & $(0.61)$ \\
\hline & \multirow{2}{*}{ No } & mean & 3.85 & 3.82 & 3.82 & 3.78 \\
\hline & & (std) & $(0.60)$ & $(0.60)$ & $(0.60)$ & $(0.61)$ \\
\hline \multirow{4}{*}{$4 \times 10^{-5}$} & \multirow{2}{*}{ Yes } & mean & 4.19 & 3.89 & 3.91 & 3.66 \\
\hline & & (std) & $(0.53)$ & $(0.52)$ & $(0.48)$ & $(0.49)$ \\
\hline & \multirow{2}{*}{ No } & mean & 3.71 & 3.68 & 3.68 & 3.63 \\
\hline & & (std) & $(0.48)$ & $(0.48)$ & $(0.48)$ & $(0.48)$ \\
\hline
\end{tabular}

Table 3.3: The mean and standard deviation of the PnL from the various strategies as the inventory-management parameter $\phi$ increases, $\varepsilon=0.04$ and 0.02 , and final inventory liquidation costs are 1 bps per share. Recall that only the benchmark HFT, who uses $\rho=0.3$, is able to correctly specify the dynamics of short-term-alpha. 


\subsection{Conclusions}

We develop an HF trading strategy where the HFT uses her superior speed advantage to process information and to send orders to the LOB to profit from roundtrip trades over very short-time scales. One of our contributions is to differentiate market orders between influential and non-influential. The arrival of influential market orders increases market order activity and also affects the shape and dynamics of the LOB. On the other hand, when non-influential market orders arrive they eat into the LOB but have no effect on the demand or supply of shares in the market.

Another contribution is to model short-term-alpha in the drift of the midprice as a zeromean reverting process which jumps by a random amount upon the arrival of influential market orders and news. Influential buy and sell market orders induce short-lived upward and downward trends in the midprice of the asset (good and bad news have a similar effect). This specification allows us to capture the essence of HF trading - to exploit shortlived predictable opportunities by way of directional strategies, and to supply liquidity to the market, taking into account adverse selection costs.

The trading strategy that the HFT employs is given by the solution of an optimal control problem where the trader is constantly submitting and cancelling limit orders to maximize expected terminal wealth, while managing inventories, over a short time interval $T$. The strategy shows how to optimally post (and cancel) buy and sell orders and is continuously updated to incorporate information on the arrival of market orders, news (good, bad and ambiguous), size and sign of inventories, and short-term-alpha. The optimal strategy captures many of the key characteristics that differentiate HFTs from other algorithmic traders: profit from directional strategies based on predicting short-term-alpha; reduced exposure to limit orders being picked off by better informed traders; and strong mean reversion of inventories to an optimal level throughout the entire strategy (and to zero at the terminal time).

Our framework allows us to derive asymptotic solutions of the optimal control problem under very general assumptions on the dynamics of the LOB. We test our model using simulations where we assume different types of HFTs who are mainly characterized by the quality of the information that they are able to process and incorporate into their optimal postings. We show that those HFTs who incorporate predictions of short-term price deviations in their strategy will deliver positive expected profits. The other HFTs are driven out of the market because their limit orders are picked off by better informed traders and cannot profit from directional strategies which are also based on short-lived predictable trends. We also show that those HFTs who cannot execute profitable directional strategies and are systematically being picked off can stay in business if they exert 
tight controls on their inventories. In our model, these controls imply a higher penalty on their inventory position which pushes the optimal limit orders further away from the midprice so the chances of being picked off by other traders are considerably reduced.

One aspect that we have left unmodeled is when it is optimal for the HFT to submit market orders. We know that HFTs submit both aggressive and passive orders. Depending on short-term-alpha it might be optimal for the HFT to submit aggressive orders (for one or both legs of the trade) to complete a directional strategy. In our stochastic optimal control problem, the HFT does not execute market orders; the best she can do is send limit orders at the midprice (zero spread), but this is no guarantee that the limit order will be filled in time for the HF strategy to be profitable. We leave for future research the optimal control problem where HFTs can submit both passive and aggressive orders in this specific theoretical context. Market orders are included in the application of this chapter's MM strategy to real data in Section 6.3 and the multiple asset HFT problem posed in Chapter 4.

Finally, the self-exciting nature of our model captures other important features of strategic behavior which include "market manipulation". For example, algorithms could be designed to send market orders, in the hope of being perceived as influential, to trigger other algorithms into action and then profit from anticipating the temporary changes in the LOB and short-term-alpha. Market manipulation strategies are not new to the marketplace; they have been used by some market participants for decades. Perhaps what has changed is the speed at which these techniques are executed and the question is whether speed enhances the ability to go undetected. Analyzing such strategies is beyond the scope of this thesis. 


\section{Chapter 4}

\section{Algorithmic Trading in Multiple Assets}

\subsection{Introduction}

There has been extensive research done in single asset optimal trading. Whether it is in an optimal liquidation/acquisition framework as in Almgren and Chriss (2000), Almgren (2003), Kharroubi and Pham (2010), Guéant et al. (2012), and Bayraktar and Ludkovski (2012), or in a general profit/utility maximization setting as in Avellaneda and Stoikov (2008), Guilbaud and Pham (2013b), and Cartea et al. (2014), single asset high frequency trading (HFT) control problems have been studied in a variety of flavours. In contrast to these, we develop an optimal trading strategy in the presence of multiple assets that are not only correlated, but present static arbitrage opportunities if their midprices stray outside the no-arbitrage region. This will be made precise later and the size/shape of this region will be dictated by the assets' bid/offer spreads.

The framework presented in this chapter is motivated by the following illustrative example. Suppose that an investor maximizes expected wealth by trading in a futures which matures at time $T_{1}$ and also trades in its underlying. Further assume that this underlying equity does not pay dividends and the risk-free rate is zero. Let $S_{t}^{1}$ denote the price of the underlying equity at time $t$ and $S_{t}^{2}$ the price of the futures contract. In an economy where assets are traded with a zero bid-ask spread (that is, the price to sell or buy the asset is the same), we have, by no-arbitrage, $S_{t}^{1}=S_{t}^{2}$ for all $t \leq T_{1}$. If this equality does not hold, then there exists an arbitrage.

Now assume that an underlying equity and its futures contract trade with bid-ask spreads 
$\xi_{1}>0$ and $\xi_{2}>0$, respectively. In this case, it is possible for the market to bear arbitragefree prices of the form $S_{t}^{1} \neq S_{t}^{2}$. To see this, assume that $S_{t}^{1}>S_{t}^{2}$. To arbitrage these prices the investor sends a buy market order (MO) for the futures contract, for which she must cross the spread and pay $S_{t}^{2}+\xi_{2} / 2$, and simultaneously sends a sell MO for the underlying and obtains $S_{t}^{1}-\xi_{1} / 2$. This trade is an arbitrage if $\left(S_{t}^{1}-\xi_{1} / 2\right)-\left(S_{t}^{2}+\xi_{2} / 2\right)>0$. Hence, it is simple to show that the no-arbitrage region is $\left|S_{t}^{1}-S_{t}^{2}\right| \leq\left(\xi_{1}+\xi_{2}\right) / 2$.

Similarly, suppose we want to make markets in the following three derivatives: The first nearby futures contract written on an equity having maturity $T_{1}$, the second nearby futures contract written on the same equity having maturity $T_{2}>T_{1}$, and the 1-2 calendar spread contract ${ }^{39}$ having maturity $T_{1}$. If an investor wants to trade all three assets, and is concerned with holding risky positions for any period of time, then she should try to take advantage of the fact that some of these assets are strongly correlated with each other. Furthermore, these three assets are more than just correlated. By construction of the calendar spread contract, this economy has more structure than that of one that is just governed by correlated diffusion processes.

In this chapter, we have an investor who wishes to make markets via limit orders (LOs) in a mutliple asset economy. In addition to this, the investor may trade aggressively and take liquidity via market orders if she sees a profitable opportunity in the form of a statistical arbitrage. The goal is to maximize cash profits at some terminal time $T$, while controlling for inventory risk by penalizing open positions.

We show that the optimal trading strategy has two active components. When sufficiently far from the no-arbitrage boundary, the investor makes markets and prefers to hold no inventory, or some combination of the assets that has little risk. When the midprice is reasonably close to the no-arbitrage boundary, the investor will adjust her limit quote depth and possibly execute market orders to advantage of this statistical arbitrage opportunity.

Inventory control also plays a crucial role in the optimal trading strategy. Optimal LO depth as a function of inventory and a graphical representation of the continuation region will present the reader with financial intuition as to how this strategy is qualitatively operating. We also test our strategy against simulated data for a number of values of the inventory penalty parameter $\phi$, which yields an efficient frontier of trading strategies.

Finally, an explicit numerical scheme is provided to solve the resulting Hamilton-JacobiBellman (HJB) quasi-variational inequality (QVI) on a discrete grid in time and space,

\footnotetext{
${ }^{39}$ The 1-2 calendar spread is a derivative that forces one party to exchange the $T_{2}$ maturity contract for the underlying asset at time $T_{1}$. Its payoff can be decomposed into being long the $T_{1}$ contract and short the $T_{2}$ contract.
} 
as well as a dimensional reduction in the spatial variables. We prove that this scheme indeed converges to viscosity solution of the HJBQVI.

This chapter is organized as follows: Section 4.2 contains definitions and some modeling assumptions, Section 4.3 states and discusses properties of the optimal trading strategy, Section 4.4 details a simulation study and the performance of various strategies are analyzed, Section 4.5 discusses the discretization scheme and convergence to the viscosity solution, and Section 4.6 is for conclusions.

\subsection{The Model}

Here we develop a framework for investors who trade in a basket of assets. Our main focus is on assets that exhibit strong structural dependencies as in the example above where the investor trades in a futures contract and the underlying. The main question we answer is: How can an investor design an algorithmic strategy to take advantage of this structural dependency between assets?

We assume that the economy trades $n$ risky assets with midprices at time $t$ given by $S_{t}^{i}, i=1, \cdots, n$, and respective bid-offer spreads $\xi_{i}>0$, both of which we arrange in $n$-dimensional vectors $\mathbf{S}_{t}$ and $\boldsymbol{\xi}$ respectively. We also assume the existence of a cash asset (i.e., risk-free asset that offers zero return) which trades with zero bid-ask spread.

In classical works in quantitative finance, when one asset's payoff is just a linear combination of the others, then the economy would contain a redundant asset. However, when some of these assets trade with a bid-offer spread, this redundancy ceases to exist. We classify a multiple asset economy as over-complete if there exists a non-zero portfolio and a finite investment horizon $\bar{T}$ such that this portfolio matures at a constant.

Definition 4.2.1. A financial system is said to be over-complete if $\exists \mathbf{b} \in \mathbb{R}^{n}, \bar{T}>0$ with $\|\mathbf{b}\|>0$ such that $\mathbb{V}\left[\mathbf{b} \cdot \mathbf{S}_{\bar{T}}\right]=0$, provided $\mathbf{S}_{\bar{T}} \in L^{2}\left(\Omega, \mathcal{F}_{\bar{T}}, \mathbb{P}\right)$, where $\mathbb{V}$ is the variance operator, and the operation $\cdot$ is the dot product. ${ }^{40}$

Our motivating example (first two nearby futures and the calendar spread) fits into this framework with portfolio $\mathbf{b}=(1,-1,-1)$ and $\bar{T}$ being the maturity of the first nearby futures. The illustrative example that will be used throughout this chapter (first nearby

\footnotetext{
${ }^{40}$ Unless otherwise stated, all random variables and stochastic processes in this chapter are defined on the completed, filtered probability space $\left(\Omega, \mathcal{F}_{\bar{T}}, \mathbb{F}, \mathbb{P}\right)$ with filtration $\mathbb{F}=\left\{\mathcal{F}_{t}\right\}_{0 \leq t \leq T}$ and where $\mathbb{P}$ is the real-world probability measure. Note that the filtration $\mathbb{F}$ is only defined up to time $T<\bar{T}$, whereas $\mathbb{P}$ is defined on the larger $\sigma$-algebra $\mathcal{F}_{\bar{T}}$. In this chapter, $\mathbb{F}$ is the natural filtration generated by $\left(\mathbf{S}_{t}, \mathbf{M}_{t}^{ \pm}, \mathbf{N}_{t}^{ \pm}\right)$, all of which will be defined later.
} 
futures and the underlying equity) also fits into this framework with $\mathbf{b}=(1,-1)$ and $\bar{T}$ being the maturity of the futures contract.

In Definition 4.2.1, the trivial portfolio $\mathbf{b}=\mathbf{0}$ is excluded from $\mathbb{R}^{n}$ since $\mathbb{V}\left[\mathbf{0} \cdot \mathbf{S}_{\bar{T}}\right]$ would always be zero, making every economy over-complete and rendering this distinction trivial. Notice that if the economy does not admit such a portfolio (i.e. there does not exist such a vector $\mathbf{b}$ ), then this problem reduces to a multiple asset problem with correlated assets. The no-arbitrage region in this simplified case would be all of $\mathbb{R}^{n}$. Also observe that the set from Definition 4.2.1 consists of all the riskless portfolios when considering $\bar{T}$ as the investment horizon. However, this may be the only investment horizon where these portfolios are riskless.

Definition 4.2.2. Define the space $B$ as the set of all vectors $\mathbf{b}$ from Definition 4.2.1 plus the origin. Observe that $B$ is a subspace of $\mathbb{R}^{n}$.

We require the set $B$ in Definition 4.2.2 to include the origin so that it is, in fact, a subspace. In the simplified case where the economic system is not over-complete, the set $B$ would only consist of the origin.

Definition 4.2.3. The no-arbitrage region, denoted $\mathcal{A} \subset \mathbb{R}^{n}$, is the set of midprices that makes this economy arbitrage free.

It is worthy to point out that the no-arbitrage region is open. More specifically, $\mathcal{A}$ is the set of midprices such that the initial cost to purchase a portfolio $\mathbf{b}$ minus the terminal payoff, $\mathbf{b} \cdot \mathbf{S}_{\bar{T}}$, is strictly negative, whenever this portfolio matures at a constant value (i.e. when $\mathbb{V}\left[\mathbf{b} \cdot \mathbf{S}_{\bar{T}}\right]=0$ ).

This observation deserves some explanation. It is clear that the no-arbitrage set $\mathcal{A}$, as previously described, is open. So what happens on the boundary? These portfolios have cost equal to their maturity value. So how exactly is it an arbitrage? It has the potential for profits via the following strategy: Obtain portfolio $\mathbf{b}$ now for $\operatorname{cost} \mathbf{b} \cdot \mathbf{S}_{\bar{T}}$. There is a positive probability that prior to $\bar{T}, \mathbf{S}_{t}$ will move to a value (within $\mathcal{A}$ ) such that a filled limit quote on one asset combined with liquidation of the others assets via market orders produces a net gain. Because there is a positive probability that such an opportunity does not arise, having initial cost strictly less than $\mathbf{b} \cdot \mathbf{S}_{\bar{T}}$ is enough to ensure it is not an arbitrage portfolio. This is due to the fact that if the agent can only execute market orders, the portfolio $\mathbf{b}$ (which costs $\mathbf{b} \cdot \mathbf{S}_{\bar{T}}$ to obtain) can be liquidated for exactly $\mathbf{b} \cdot \mathbf{S}_{\bar{T}}$ only in the best case scenario (on the opposite boundary). In fact, this scenario is only in the closure of $\mathcal{A}$, and hence the midprice process can only move arbitrarily close to it. Notice that the only assumption we have made here is that the spreads are positive constants. 
In the 2-dimensional illustrative example consisting of a futures contract and its underlying, we have $\mathcal{A}=\left\{\mathbf{s} \in \mathbb{R}^{2}:\left|s_{1}-s_{2}\right|<\frac{1}{2}\left(\xi_{1}+\xi_{2}\right)\right\}$. That is, at any time $t<\bar{T}$, the midprices of these two assets cannot differ by more than the sum of their half-spreads; if they do, then one could execute two market orders, for example one order to short the overpriced futures and the other to purchase the underpriced underlying, and hold to maturity for an arbitrage.

Given the discussions above regarding the no-arbitrage region, the investor assumes that for $t \in[0, T]$, the dynamics of the midprice process $\mathbf{S}_{t}$ is a reflected, correlated, driftless Brownian Motion. However, the true underlying dynamics of the data generating process (DGP), which includes adverse selection effects, are discussed in Section 4.4.1 and used in the simulation study.

Assumption 4.2.4. The n-dimensional midprice process $\left\{\mathbf{S}_{t}\right\}_{0 \leq t \leq \bar{T}}$ has the following dynamics, for $t \in[0, T]$, on the open set $\mathcal{A}$ :

$$
d \mathbf{S}_{t}=\boldsymbol{\sigma} d \mathbf{B}_{t}
$$

with $\boldsymbol{\sigma}$ being an n-by-n matrix with $\boldsymbol{\sigma} \boldsymbol{\sigma}^{\prime}$ being positive definite, $\left\{\mathbf{B}_{t}\right\}_{0 \leq t \leq T}$ is a standard Brownian Motion on $\mathbb{R}^{n}$, and $\mathbf{S}_{t}$ has (normal) reflection at the boundary of $\mathcal{A}$.

When the risky assets possess a structural dependence, as in our illustrative example, the matrix $\boldsymbol{\sigma}$ will induce a strong correlation between all or some of the $n$ assets. For example, the case discussed above consisting of $n=2$, a futures contract and its underlying, $\boldsymbol{\sigma}$ will induce a strong positive correlation. In general, for assets that exhibit strong structural dependence, $\boldsymbol{\sigma}$ will induce movements in the midprice processes that are relatively in the same direction, on average, to some element of the subspace $B^{\perp}$ (the orthogonal compliment of $B$ ).

Recall that the agent will be submitting both limit orders and market orders and therefore needs to model incoming market orders from other participants in addition to her own executions (which she controls). As such, she assumes that other market participants send market orders according to independent Poisson processes. The counting processes representing the total number of market orders submitted by other agents up until time $t$ are $\left\{N_{i, t}^{-}\right\}_{0 \leq t \leq T}$ for sells and $\left\{N_{i, t}^{+}\right\}_{0 \leq t \leq T}$ for buys. On the other hand, the total number of market sells and buys up until time are given by the processes $\left\{\bar{N}_{i, t}^{-}\right\}_{0 \leq t \leq T}$ and $\left\{\bar{N}_{i, t}^{+}\right\}_{0 \leq t \leq T}$ respectively. It is also convenient to have these processes in the following vector forms: $\mathbf{N}_{t}^{ \pm}=\left(N_{1, t}^{ \pm}, \ldots, N_{n, t}^{ \pm}\right)^{\prime}$ and $\overline{\mathbf{N}}_{t}^{ \pm}=\left(\bar{N}_{1, t}^{ \pm}, \ldots, \bar{N}_{n, t}^{ \pm}\right)^{\prime}$. Finally, the total number of market orders sent by the high frequency trader (HFT) up to time $t$ is $\overline{\mathbf{N}}_{t}^{ \pm}-\mathbf{N}_{t}^{ \pm}$.

Thus, $N_{t}=\sum_{i=1}^{n}\left(N_{i, t}^{-}+N_{i, t}^{+}\right)$is the total number of market orders executed by other 
agents up to time $t$, and $\bar{N}_{t}=\sum_{i=1}^{n}\left(\bar{N}_{i, t}^{-}+\bar{N}_{i, t}^{+}\right)$to be the total number of market orders executed by all agents up to time $t$. It is also convenient to have these processes in the following vector forms: $\mathbf{N}_{t}^{ \pm}=\left(N_{1, t}^{ \pm}, \ldots, N_{n, t}^{ \pm}\right)^{\prime}$ and $\overline{\mathbf{N}}_{t}^{ \pm}=\left(\bar{N}_{1, t}^{ \pm}, \ldots, \bar{N}_{n, t}^{ \pm}\right)^{\prime}$. Thus, the total number of market orders sent by the market maker up to time $t$ is $\bar{N}_{t}-N_{t}$.

The investor sends LOs to the limit order book (LOB) at depth $\delta_{t}^{i}, i=1, \cdots, n$, and we summarize this information in the (2n-dimensional) vector-valued process $\left\{\boldsymbol{\delta}_{t}\right\}_{0 \leq t \leq T}$. Here we use the convention that LO depth is from across the book; the limit buy order is measured from the best offer, and the limit sell order is measured from the best bid quote. Therefore, the investor's buy LOs would be posted at $\mathbf{S}_{t}+\boldsymbol{\xi} / 2-\boldsymbol{\delta}_{t}^{-}$, and her sell LOs would be posted at $\mathbf{S}_{t}-\boldsymbol{\xi} / 2+\boldsymbol{\delta}_{t}^{+}$. In addition, the running counts of the investor's filled LOs are given by the processes $\left\{M_{i, t}^{+}\right\}_{0 \leq t \leq T}$ and $\left\{M_{i, t}^{-}\right\}_{0 \leq t \leq T}$ for sell and buy orders, respectively, and we summarize this information in the vector-valued processes $\left\{\mathbf{M}_{t}^{+}\right\}_{0 \leq t \leq T}$ and $\left\{\mathbf{M}_{t}^{-}\right\}_{0 \leq t \leq T}$. This allows the investor to post inside the spread. ${ }^{41}$ With this in mind, the investor's buy LOs would be posted at $\mathbf{S}_{t}+\boldsymbol{\xi} / 2-\boldsymbol{\delta}_{t}^{-}$, and her sell LOs would be posted at $\mathbf{S}_{t}-\boldsymbol{\xi} / 2+\boldsymbol{\delta}_{t}^{+}$.

Assumption 4.2.5. The fill rate functions for asset $i=1, \ldots, n$, as measured from "across the book", and denoted $h_{i}^{ \pm}(\delta)$, satisfy $h_{i}^{ \pm}(0)<\infty, h_{i}^{ \pm}\left(\xi_{i}\right)=\lambda_{i}^{ \pm}$, and $\lim _{\delta \rightarrow \infty} \delta h_{i}^{ \pm}(\delta)=$ 0. Furthermore, $h_{i}^{ \pm} \in C^{1}([0, \infty))$ with $h^{\prime}<0$.

The investor gets filled when MOs arrive and reach the level that the limit quote is posted at. More specifically, the deeper in the book the agent posts, the less frequent she gets filled by a matching MO; a dependence that is captured by the functions $h_{i}^{ \pm}(\delta)$. This is in line with how a number of other authors have modeled optimal postings and fill probabilities as in Ho and Stoll (1981), Avellaneda and Stoikov (2008), Bayraktar and Ludkovski (2012), Cartea and Jaimungal (2013b) and Guéant et al. (2013). This approach can be viewed as a reduced form one, in contrast to models which focus on modeling the dynamics of each level of the LOB, together with market order arrivals (see, e.g., Roşu (2009) and Cont et al. (2010)).

We may suppress the \pm and $i$ clarifiers on the function $h$ and the scalar variables $\lambda, \kappa$ (defined in Example 4.3.3), $\delta$, and $\xi$, when it is clear which element is being represented.

\footnotetext{
${ }^{41}$ Inside the spread refers to LOs that improve upon the current best bid or offer.
} 


\subsection{The HFT's Optimization Problem}

\subsubsection{Statement of the Problem}

Recall that $\boldsymbol{\delta}_{t}$ is the depth, as measured from across the book, at which the investor sends quotes to the LOB. Let $(\boldsymbol{\tau} ; \boldsymbol{\varsigma})=\left\{\tau_{1}, \tau_{2}, \ldots ; \varsigma_{1}, \varsigma_{2}, \ldots\right\}$ be an impulse control representing the investor's submission of market orders to the LOB. The ordered sequence of uniformly bounded $\mathcal{F}_{t}$ stopping times, $0 \leq \tau_{1} \leq \tau_{2} \leq \cdots \leq T$, is the sequence of times of the market orders, and the binary sequence $\varsigma_{1}, \varsigma_{2}, \ldots$ indicates whether the MO was a buy or a sell order. Recall that the filtration $\left\{\mathcal{F}_{t}\right\}_{0 \leq t \leq T}$ is generated by the processes $\mathbf{S}_{t}, \mathbf{M}_{t}^{ \pm}, \mathbf{N}_{t}^{ \pm}, \overline{\mathbf{N}}_{t}^{ \pm}$.

The cash process of the investor is then given by

$$
\begin{aligned}
d X_{t}= & \left(\mathbf{S}_{t}-\boldsymbol{\xi} / 2+\boldsymbol{\delta}_{t-}^{+}\right) d \mathbf{M}_{t}^{+}-\left(\mathbf{S}_{t}+\boldsymbol{\xi} / 2-\boldsymbol{\delta}_{t-}^{-}\right) d \mathbf{M}_{t}^{-} \\
& +\left(\mathbf{S}_{t}-\boldsymbol{\xi} / 2\right) d\left(\overline{\mathbf{N}}_{t}^{-}-\mathbf{N}_{t}^{-}\right)-\left(\mathbf{S}_{t}+\boldsymbol{\xi} / 2\right) d\left(\overline{\mathbf{N}}_{t}^{+}-\mathbf{N}_{t}^{+}\right),
\end{aligned}
$$

where $\mathbf{S}_{t}, \boldsymbol{\delta}_{t}^{-}, \boldsymbol{\delta}_{t}^{+}$, and $\boldsymbol{\xi}$ are all $n$-dimensional row vectors, and $\mathbf{M}_{t}^{-}, \mathbf{M}_{t}^{+}, \mathbf{N}_{t}^{-}, \mathbf{N}_{t}^{+}, \overline{\mathbf{N}}_{t}^{-}$, and $\overline{\mathbf{N}}_{t}^{+}$are all $n$-dimensional column vectors.

The terms in the first line in (4.2) of the cash process account for the changes in cash due to filled limit sell and buy orders and the terms in the second line account for changes in cash due to the investor's own market sell and buy orders.

The investor seeks the strategy $\left(\left\{\boldsymbol{\delta}_{t} \geq 0\right\}_{0 \leq t \leq T}, \boldsymbol{\tau}, \boldsymbol{\varsigma}\right)$ which maximizes her expected terminal wealth while penalizing and constraining inventories, where $\boldsymbol{\delta}_{t}$ is a bounded, $\mathcal{F}_{t}$ previsible function representing the LO quote depths, $\boldsymbol{\tau}$ is an increasing sequence of $\mathcal{F}_{t}$ stopping times representing the times of MO submissions, and $\boldsymbol{\varsigma}$ is the binary sequence of buy/sell indicators. The collection of all such strategies is called the admissible set and denoted by $\mathcal{X}$.

Thus, for $0<T \ll \bar{T}$, the investor solves the control problem

$$
\Phi(t, x, \mathbf{s}, \mathbf{q})=\sup _{\left(\boldsymbol{\delta}_{t}, \boldsymbol{\tau}, \boldsymbol{\varsigma}\right) \in \mathcal{X}} \mathbb{E}_{(t, x, \mathbf{s}, \mathbf{q})}\left[X_{T}+\sum_{i=1}^{n} q_{T}^{i}\left(S_{T}^{i}-\operatorname{sign}\left(q_{T}^{i}\right) \frac{\xi_{i}}{2}-\gamma q_{T}^{i}\right)-\phi \int_{t}^{T} \ell\left(\mathbf{q}_{u}\right) d u\right]
$$

where $\gamma \geq 0, \phi \geq 0, \mathbf{q}_{t}=\left(q_{t}^{1}, \ldots, q_{t}^{n}\right)$ is the investor's inventory at time $t$, and $\ell(\cdot) \geq 0$ is an instantaneous inventory penalty.

In Equation (4.3), we have included two inventory penalties. The first of which is a cost that the investor incurs to unwind terminal inventory and the other is a running 
inventory penalty which is used to control the strategies exposure to inventory risk, but not a financial cost. When the strategy reaches its terminal time $T$, inventories must be liquidated. To do so the investor uses a market order that crosses the spread, picks up the $\operatorname{cost} \xi_{i} / 2$, and walks down one side of the LOB where these costs are given by the linear "market impact" term $\gamma q_{T}^{i}$.

On the other hand, the running inventory penalty $\phi \int_{t}^{T} \ell\left(\mathbf{q}_{u}\right) d u$ is imposed by the investor to adjust the strategy's exposure to inventory risk, see Cartea and Jaimungal (2013b) and Cartea et al. (2013). Here we assume that

$$
\ell(\mathbf{q})=\mathbb{V}\left[\mathbf{q} \cdot \widehat{\mathbf{S}}_{1}\right]=\mathbf{q}^{\prime} \boldsymbol{\sigma} \boldsymbol{\sigma}^{\prime} \mathbf{q},
$$

where $\left\{\widehat{\mathbf{S}}_{t}\right\}_{0 \leq t \leq T}$ is the unreflected version of $\left\{\mathbf{S}_{t}\right\}_{0 \leq t \leq T}$.

A priori, it is unclear why the unreflected version of $\mathbf{S}_{t}$ in the penalty function (4.4) is used. Classical mean-variance portfolio optimization problems penalize according to the variance of the terminal profit and loss $(\mathrm{PnL})$. However, such a penalty fails to characterize how much inventory risk was taken on by the investor throughout the entire life of the strategy. In light of this shortfall, we take the penalty to be the intra-trade quadratic variation of the investor's portfolio, similar to what was done in Forsyth et al. (2012), which is a natural characterization of the total risk taken on by the investor. The following observation gives a mathematical justification for the use of the inventory penalty in (4.3).

Observation 4.3.1. For $0 \leq u \leq v \leq T$, we have the following equivalence ( $\mathbb{P}$ almost surely):

$$
\int_{u}^{v} \mathbb{V}\left[\mathbf{q}_{t} \cdot \widehat{\mathbf{S}}_{1}\right] d t=\langle\mathbf{q} \cdot \mathbf{S}\rangle_{v}-\langle\mathbf{q} \cdot \mathbf{S}\rangle_{{\overline{N_{N}}}_{v}}+\sum_{k=\bar{N}_{u}+1}^{\bar{N}_{v}}\left\{\langle\mathbf{q} \cdot \mathbf{S}\rangle_{t_{k}-}-\langle\mathbf{q} \cdot \mathbf{S}\rangle_{t_{k-1}}\right\}
$$

where $t_{k}$ is the time of the $k^{\text {th }}$ market order, $t_{k}-$ is the left limit at $t_{k}$, and $\langle\cdot\rangle_{t}$ is the quadratic variation operator.

The relation in (4.5) is also highlighted in Cartea and Jaimungal (2013b) where the authors offer a similar relation for the one-dimensional case. Thus, the trader penalizes the strategy with the "variance per second" of her current portfolio, which gets scaled by $\phi \geq 0$ according to her risk tolerance. In a single asset economy, this penalty reduces to $\int q_{u}^{2} d u$ (up to a multiplicative constant), which has been shown in Cartea et al. (2013) to stem from ambiguity aversion the drift of the midprice process. 


\subsubsection{Solving the Value Function}

By appealing to Øksendal and Sulem (2007) and the results in Appendix B.1, the value function $\Phi(t, x, \mathbf{s}, \mathbf{q})$, as defined in (4.3), then satisfies the following HJBQVI on the no-arbitrage region $\mathcal{A}$ :

$$
\begin{aligned}
& \max \left[\sup _{\boldsymbol{\delta}>0}\left\{\left(\partial_{t}+\mathcal{L}\right) \Phi+\sum_{i} h_{i}^{-}\left(\delta_{i}^{-}\right) \Delta_{i}^{-} \Phi+\sum_{i} h_{i}^{+}\left(\delta_{i}^{+}\right) \Delta_{i}^{+} \Phi-\phi \ell(\mathbf{q})\right\}\right. ; \\
&\left.\max _{i, \pm}\left\{\Phi\left(t, x \pm s_{i}-\xi_{i} / 2, \mathbf{s}, \mathbf{q} \mp e_{i}\right)-\Phi\right\}\right]=0
\end{aligned}
$$

with time boundary condition $\Phi(T, \cdot)=x+\sum_{i=1}^{n} q_{i}\left(s_{i}-\operatorname{sign}\left(q_{i}\right) \frac{\xi_{i}}{2}-\gamma q_{i}\right)$, the Neumann condition on the boundary of $\mathcal{A}$

$$
\frac{\partial \Phi}{\partial \boldsymbol{n}}=\nabla \Phi \cdot \boldsymbol{n}=0
$$

where $\boldsymbol{n}$ is the normal vector to $\partial \mathcal{A}$, and the infinitesimal generator $\mathcal{L}$ of the $n$-dimensional correlated Brownian Motion is given by $\frac{1}{2} \sum_{i, j=1}^{n}\left[\boldsymbol{\sigma} \boldsymbol{\sigma}^{\prime}\right]_{i j} \frac{\partial^{2}}{\partial s_{i} \partial s_{j}}$.

Moreover, here we use $\Delta_{i}^{ \pm}$to denote the difference operator

$$
\Delta_{i}^{ \pm} \varphi(t, x, \mathbf{s}, \mathbf{q})=\varphi\left(t, x \pm s_{i} \pm \delta_{i}^{ \pm} \mp \xi_{i} / 2, \mathbf{s}, \mathbf{q} \mp e_{i}\right)-\varphi(t, x, \mathbf{s}, \mathbf{q})
$$

where $e_{i}$ is a vector with 1 in the $i^{\text {th }}$ position and 0 elsewhere, and dependence on $\boldsymbol{\delta}$ is suppressed for convenience. For example, $\Delta_{i}^{-} \varphi$ denotes the change in $\varphi$ due to the investor's limit buy order being filled: inventory for asset $i$ increases by 1 , and cash $x$ decreases by the cost of the transaction $s_{i}-\delta_{i}^{-}+\xi_{i} / 2$. The interpretation for $\Delta_{i}^{+} \varphi$ when the investor's limit sell order is filled has a similar interpretation.

Lemma 4.3.2. If the the midprice process is in the no-arbitrage region (i.e. $\mathbf{s} \in \mathcal{A}$ ), then the supremum term in (4.6) is equal to the sum of the supremum over each component.

Proof. The supremum operator satisfies countable sub-additivity. Equality comes from the fact that the $2 n$ variables being optimized appear exactly once in all of $2 n$ distinct additive terms.

We apply the following ansatz for the value function

$$
\Phi(t, x, \mathbf{s}, \mathbf{q})=x+\mathbf{q} \cdot \mathbf{s}+g(t, \mathbf{s}, \mathbf{q})
$$


and recalling that $h(\delta)$ is the fill rate function facing the investor for an LO placed at depth $\delta$, the first order condition for each component of $\boldsymbol{\delta}$ in the continuation region is given by

$$
h^{\prime}\left(\delta_{i}^{ \pm, *}\right)\left(\delta_{i}^{ \pm, *}-\frac{\xi_{i}}{2}+\Delta_{i}^{ \pm} g\right)+h_{i}^{ \pm}\left(\delta_{i}^{ \pm, *}\right)=0 .
$$

The implicit form in Equation (4.9) is then used to simplify (4.6) and obtain a new QVI for $g(\cdot)$ :

$$
\max \left[\left(\partial_{t}+\mathcal{L}\right) g-\sum_{i, \pm} \frac{\left(h\left(\delta^{*}\right)\right)^{2}}{h^{\prime}\left(\delta^{*}\right)}-\phi \ell(\mathbf{q}), \max _{i, \pm}\left\{\Delta_{i}^{ \pm} g-\xi_{i} / 2\right\}\right]=0
$$

with boundary conditions $g(T, \cdot)=-\sum q_{i}\left(\operatorname{sign}\left(q_{i}\right) \frac{\xi_{i}}{2}+\gamma q_{i}\right)$ and $(\mathbf{q}+\nabla g) \cdot \boldsymbol{n}=0$ on $\partial \mathcal{A}$.

In order to obtain an explicit form for $\boldsymbol{\delta}^{*}$ (in feedback control form), we further assume that $h(\delta)$ is exponential.

Example 4.3.3. Exponential Fill Rate. Suppose that market orders arrive to the market according to a Poisson process and the probability of being filled decays exponentially. ${ }^{42}$ More precisely, $h_{i}^{ \pm}(\delta)=\lambda_{i}^{ \pm} e^{-\kappa_{i}^{ \pm}\left(\delta-\xi_{i}\right)}$ with $0<\kappa_{i}^{ \pm} \leq \frac{1}{\xi_{i}}$.

Example 4.3.3 provides an additional modeling assumption ${ }^{43}$ on the fill rate facing the investor that will be used in all numerical demonstrations throughout this chapter. The additional condition that $\kappa_{i}^{ \pm} \leq \frac{1}{\xi_{i}}$ is imposed so that the risk-neutral investor following an optimal market making strategy does not post limit quotes inside the spread. This condition is consistent with what is observed in the market as the smaller the bid-offer spread, the more volume rests in the LOB. This corresponds to higher values of $\kappa_{i}^{ \pm}$.

In this exponential fill rate setting, we have

$$
\delta_{i}^{ \pm, *}=\frac{1}{\kappa_{i}^{ \pm}}+\frac{\xi_{i}}{2}-\Delta_{i}^{ \pm} g
$$

and Equation (4.10), in the continuation region, further reduces to

$$
g_{t}+\frac{1}{2} \sum_{i, j=1}^{n}\left[\boldsymbol{\sigma} \boldsymbol{\sigma}^{\prime}\right]_{i j} \frac{\partial^{2} g}{\partial s_{i} \partial s_{j}}+\sum_{i, \pm} \frac{\lambda_{i}^{ \pm}}{\kappa_{i}^{ \pm}} \exp \left\{-1+\frac{\kappa_{i}^{ \pm} \xi_{i}}{2}+\kappa_{i}^{ \pm} \Delta_{i}^{ \pm} g\right\}-\phi \ell(\mathbf{q})=0 .
$$

The optimal LO depth has two main components. The first is given by the two first terms

\footnotetext{
${ }^{42}$ Exponentially decaying fill probability is implied by the stronger modeling assumption that MO volumes are iid exponential random variables and that the LOB volume is flat.

${ }^{43} \mathrm{It}$ is also common for a power law function to be used as the fill rate function.
} 
on the right-hand side in (4.11) which is the optimal depth chosen by an investor who does not impose any inventory penalty, nor does she pick up any costs for unwinding terminal inventory, $\gamma=\phi=0$. The second component, given by the third term on the right-hand of the equation, is a correction to the optimal posting to account for the adjustment to the LO depth as a result of the investor's inventory management strategy.

Proposition 4.3.4. The $\max _{i, \pm}\left\{\Delta_{i}^{ \pm} g-\xi_{i} / 2\right\}$ terms in the $Q V I$ (4.10) give rise to the $L O$ spread bounds $\frac{1}{\kappa_{i}^{ \pm}}<\delta_{i}^{ \pm} \leq \frac{1}{\kappa_{i}^{ \pm}}+\xi_{i}$ for each $i=1, \ldots, n$.

When considering $\delta^{-}$, the stated lower bound is because the agent is eager to obtain another unit of the asset. Therefore, rather than posting an LO at the strict lower bound of $1 / \kappa_{i}^{ \pm}$, she executes an MO. The upper bound corresponds to posting at a deep level (since the investor does not want the asset), and if the LO is filled, the agent immediately posts an MO to unwind and attempts to make a profit. Profits in this case are not guaranteed and will depend on the resilience of the LOB. Note that $\delta_{i}^{-}(\mathbf{q})$ is arbitrarily close to $1 / \kappa_{i}^{ \pm}$if and only if $\delta_{i}^{+}\left(\mathbf{q}+e_{i}\right)$ is close to $\frac{1}{\kappa_{i}^{ \pm}}+\xi_{i}$ and similarly, $\delta_{i}^{+}(\mathbf{q})$ is arbitrarily close to $1 / \kappa_{i}^{ \pm}$if and only if $\delta_{i}^{-}\left(\mathbf{q}-e_{i}\right)$ is close to $\frac{1}{\kappa_{i}^{ \pm}}+\xi_{i}$.

We proceed to numerically solve the QVI given in (4.10) for the special case of exponential fill rate function, $h(\cdot)$. The discussion regarding the exact numerical scheme is postponed to Section 4.5, where we take advantage of a dimensional reduction in the spacial variables and also prove that the scheme converges to the unique viscosity solution.

\subsubsection{Analysis of the Optimal Trading Strategy}

We use our 2-dimensional illustrative example to explore the behaviour of the optimal strategy. Recall that the first asset $S_{t}^{1}$ is a traded equity and the second second asset, $S_{t}^{2}$, is a $\bar{T}$-maturity futures contract written on $S_{t}^{1}$. In this economy, we have $\mathbb{V}\left[(1,-1)^{\prime} \cdot \mathbf{S}_{\bar{T}}\right]=0$, yielding a market that is over-complete. To this end, we take $\mathbf{b}=(1,-1)^{\prime}$ as the basis of $B$ for this illustrative example, and other parameters are given in Table 4.1.

For simplicity, we assume that the fill rates on both sides of the LOB are the same (see Table 4.1) and that the arrival of buy and sell MOs are also the same, but both the fill rate parameter and arrival of MOs differs across the two assets. For example, the underlying equity $S_{t}^{1}$ is modeled with a higher rate of incoming market orders than $S_{t}^{2}$ which is the $\bar{T}$-maturity futures contract. This is because the underlying trades more actively than the first nearby futures and so we assume that there is more liquidity in 


\begin{tabular}{cc}
\hline \hline Parameter & Value \\
\hline$\phi$ & 1 \\
$\boldsymbol{\sigma}$ & $\left(\begin{array}{cc}0.005 & 0.009 \\
0 & 0.015\end{array}\right)$ \\
$\gamma$ & 0.001 \\
$\xi_{1}=\xi_{2}$ & 0.02 \\
$\lambda_{1}^{-}=\lambda_{1}^{+}$ & 1.2 \\
$\lambda_{2}^{-}=\lambda_{2}^{+}$ & 1.0 \\
$\kappa_{1}^{-}=\kappa_{1}^{+}$ & 40 \\
$\kappa_{2}^{-}=\kappa_{2}^{+}$ & 25 \\
$T-t$ & 60 \\
\hline \hline
\end{tabular}

Table 4.1: Model parameters used in the analysis of the function $g(t, \mathbf{q}, \mathbf{s})$, the continuation region, and the optimal inventory level. Recall that the fill rate assumption $h_{i}^{ \pm}(\delta)=\lambda_{i}^{ \pm} e^{-\kappa_{i}^{ \pm}\left(\delta-\xi_{i}\right)}$ from Example 4.3.3 is being made.

the equity than the futures by taking $\kappa_{1}^{ \pm}>\kappa_{2}^{ \pm} .{ }^{44}$

Finally, note that because $\xi_{i}>0$, we do not require their correlation to be 1 or even for their volatilities to be the same. However, it makes intuitive financial sense for their volatities to be similar and for their correlation to be positive. This leads to our choice of volatility matrix, $\boldsymbol{\sigma}$, which induces a correlation of approximately 0.87 . The total volatility of Asset 1 turns out to be less than that of Asset 2. The rationale behind this is that the sources of quadratic variation of the futures contract not only includes the underlying equity (Asset 1), but also with that of interest rates and stochastic volatility (which affects the futures price via the convexity correction; see Hull (2014)). So the futures contract should then have a larger total volatility than its underlying.

For a fixed time $t$, Figures 4.1(a) and 4.1(c) show $g(t, \mathbf{s}, \mathbf{q})$ as a function of $\mathbf{q}$ for fixed $\mathbf{s}=(100,100)$. It is clear that it attains its maximum at $\mathbf{q}=(0,0)$. The asymmetry in $g$ with respect to inventory is due to the fact that the risk associated with a single unit of Asset 1 is less than that of Asset 2. Furthermore, portfolios of the form $(a, b)$ with $\operatorname{sign}(a) \neq \operatorname{sign}(b)$ are less risky than portfolios with $\operatorname{sign}(a)=\operatorname{sign}(b)$ as the two assets are strongly positively correlated. This is depicted in the dark blue regions in the upper right and lower left corners of Figure 4.1(c), which corresponds to large long positions and large short positions in both the assets, respectively.

The left and right panels of Figure 4.1 show $g(t, \mathbf{s}, \mathbf{q})$ as a function of $\mathbf{q}$ for two different

\footnotetext{
${ }^{44}$ Although this is true for equities, there are cases (such as commodities) when a future's underlying does not actively trade or is a non-traded asset (e.g., electricity).
} 


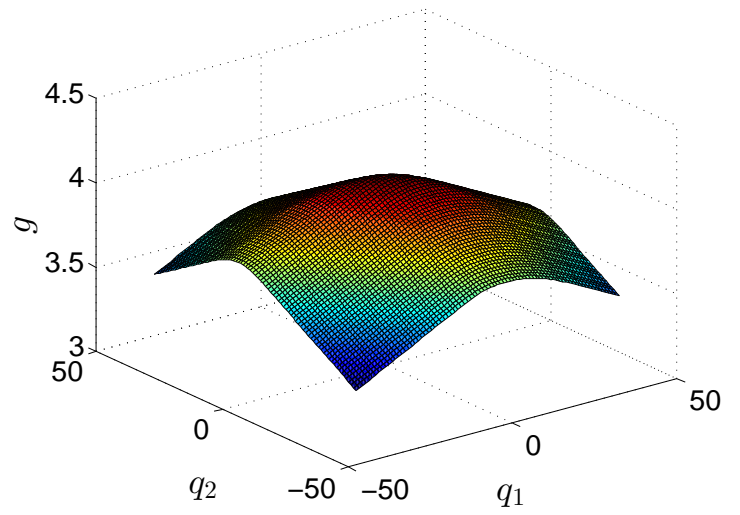

(a) $s_{1}=s_{2}$

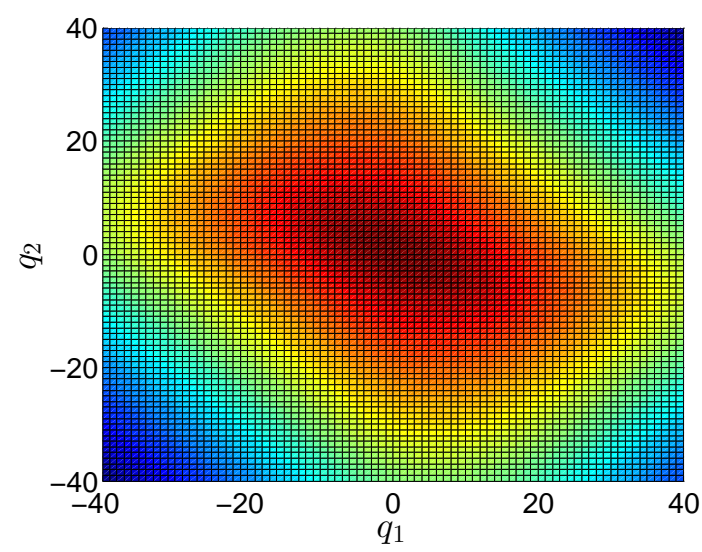

(c) $s_{1}=s_{2}$

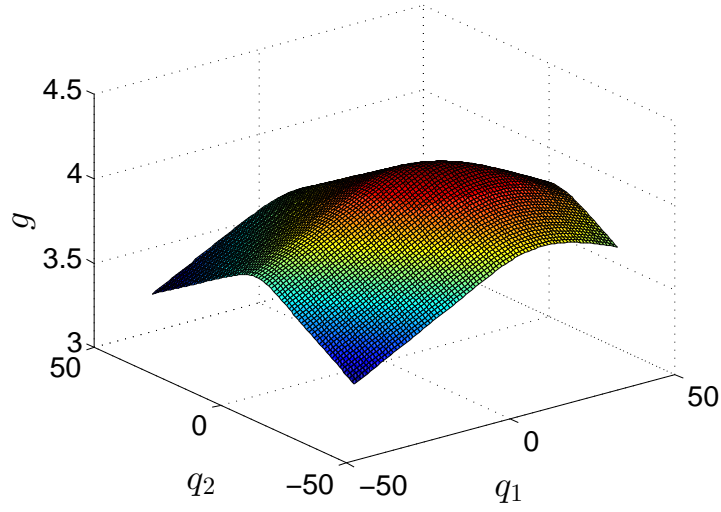

(b) $s_{1}<s_{2}$

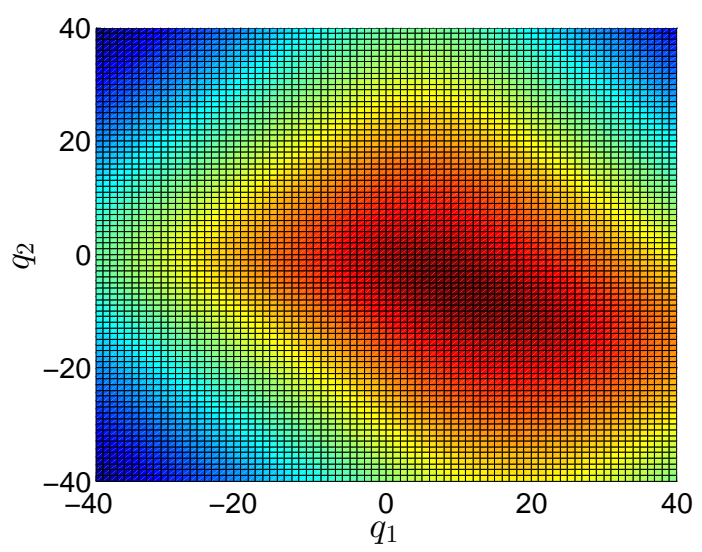

(d) $s_{1}<s_{2}$

Figure 4.1: $g$ surface for various s. Figures 4.1(a) and 4.1(c) show the surface and heatmap, respectively, of $g(t, \mathbf{s}, \mathbf{q})$ when the assets' midprices are equal. Figures $4.1(\mathrm{~b})$ and 4.1(d) show the surface and heatmap, respectively, of $g(t, \mathbf{s}, \mathbf{q})$ when Asset 1 is cheap relative to Asset $2\left(s_{1}=s_{2}-0.012\right)$.

values of $s_{1}-s_{2}$. When $s_{1}=s_{2}$, the maximum of $g(t, \mathbf{s}, \mathbf{q})$ is again obtained at $\mathbf{q}=(0,0)$. However, this is no longer the case when $s_{1} \neq s_{2}$. When $s_{1}<s_{2}$, the investor would rather be long Asset 1 and short Asset 2, and she will post LOs in a way that reflects this desire to hold such an inventory. This is because the process $S_{t}^{1}-S_{t}^{2}$ is bounded from below by the sum of the assets' half-spreads. Hence, when this process is near the lower boundary, there is the potential for profits as we have assumed reflection on this boundary.

Figure 4.2 shows how the continuation region also depends on $s_{1}-s_{2}$. When $s_{1}=s_{2}$, the continuation region is centered around the origin, just as one would expect. However, when one asset is cheap relative to the other, i.e. $s_{1}<s_{2}$, then the agent prefers to 


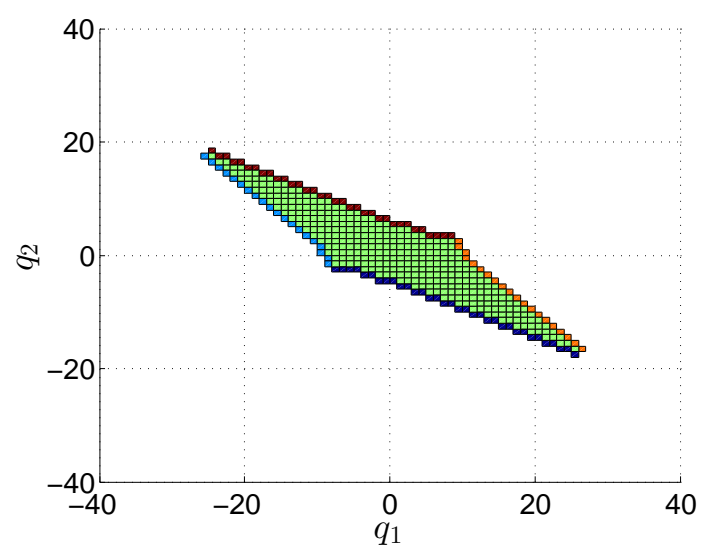

(a) $s_{1}=s_{2}$

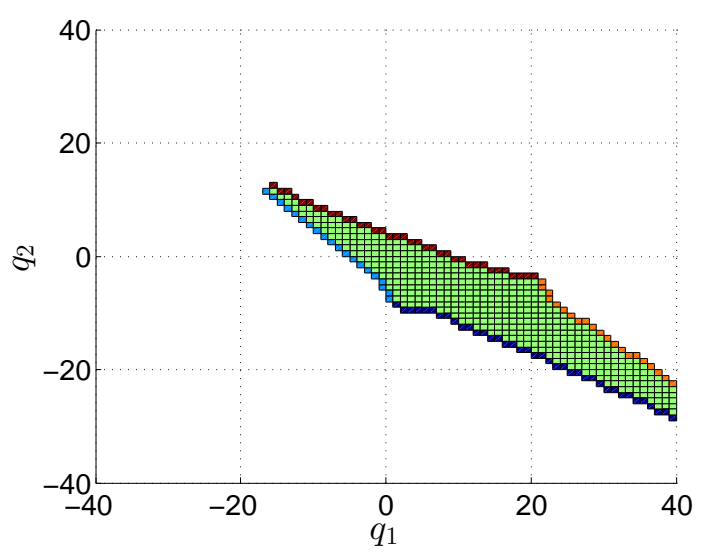

(b) $s_{1}<s_{2}$

Figure 4.2: Continuation region for various $\mathbf{s}$. The different coloured regions correspond to different MO submissions. Namely, light blue: Asset 1 buy MO; orange: Asset 1 sell MO; dark blue: Asset 2 buy MO; burgundy: Asset 2 sell MO; green: continuation region; and white: unattainable. Recall that Figure $4.2(\mathrm{~b})$ is specified by $s_{1}=s_{2}-0.012$.

long Asset 1 and short Asset 2 in an attempt to profit when Asset 1 increases relative to Asset 2. Furthermore, she will be aggressive and execute MOs if she is caught holding a portfolio that is short Asset 1 and long Asset 2 when this shift in the midprices occurs, as demonstrated by the absense of the continuation region in the upperleft corner of Figure 4.2(b). As the price discrepency becomes larger, the investor adjusts her strategy to accumulate inventory in a way that positions her to profit from these market conditions.

As discussed earlier, inventory control plays a critical role in high frequency trading. The investor actively tries to liquidate outstanding long positions and cover outstanding short positions. In a single asset scenario, the logistics behind this are clear. However, in a multiple asset setting with correlated assets, the investor may attempt to partially hedge a long position in one asset with a short position in another asset that is positively correlated.

Corollary 4.3.5. Mean Reversion of Inventory. Given the optimal strategy $\boldsymbol{\delta}^{*}$, the expected drift of the inventory process $\mathbf{q}_{t}$ inside the continuation region is given by

$$
\lim _{u \rightarrow t^{+}} \frac{1}{u-t} \mathbb{E}\left[\mathbf{q}_{u}-\mathbf{q}_{t} \mid \mathcal{F}_{t}\right]=\left[\lambda_{i}^{-} h_{i}^{-}\left(\delta_{i, t}^{-}\right)-\lambda_{i}^{+} h_{i}^{+}\left(\delta_{i, t}^{+}\right)\right]_{i} .
$$

Proof. By observing that each element of $\boldsymbol{\delta}_{t}$ is a right continuous with left limits (RCLL) function of $t$ (for fixed $\omega \in \Omega$ ), we can conclude that the expected drift in inventories inside the continuation region is given by the difference in the arrival rates of filled LOs at time $t$. 


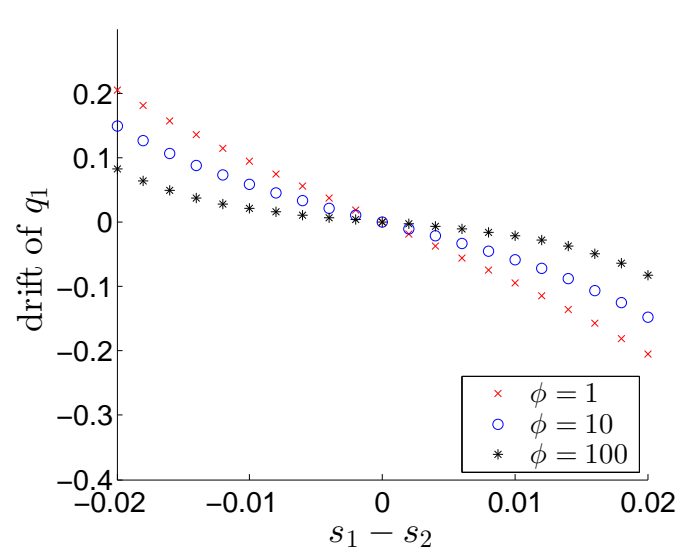

(a) Asset 1

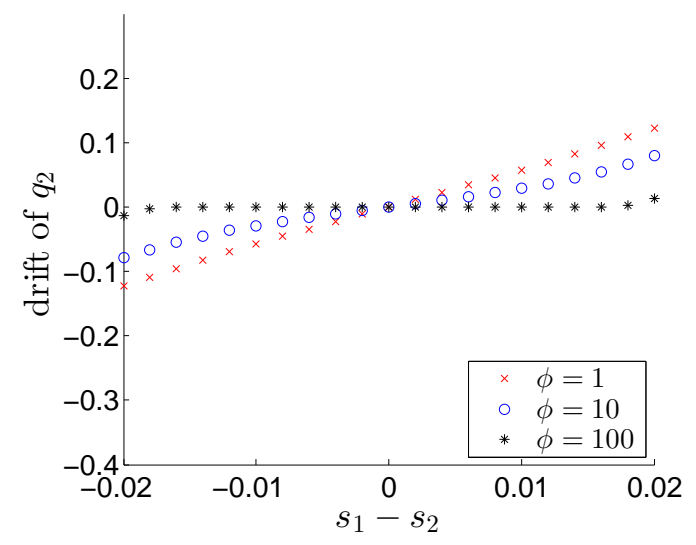

(b) Asset 2

Figure 4.3: Inventory drift when the investor is flat (i.e., $q_{1}=q_{2}=0$ ).

Figure 4.3 shows the inventory drift as a function of the midprice $\mathbf{s}$. When the trader is flat (holding zero inventory) and Asset 1 is cheap relative to Asset 2 (i.e. when $s_{1}-s_{2}$ is small), she wants to acquire a long position in Asset 1 and short position in Asset 2. The corresponding drifts in the $q_{t}^{1}$ and $q_{t}^{2}$ processes become more pronounced as the inventory penalty parameter $\phi$ decreases.

Figures 4.4 and 4.5 show the drift in the process $\mathbf{q}_{t}$ as a function of the current inventory q for fixed values of $s_{1}-s_{2}$ (and $\phi=1$ as taken in previous plots where not explicitly stated). The inventory will be attracted to the curve where the reversion rate is 0 (coloured green). Notice how in each figure the $q_{i}$ process is attracted to a curve and not a point. More specifically, for each value of $\mathbf{q}$, the component $q_{i}$ is attracted to a particular point that depends on the values of the other components of $\mathbf{q}$. When $q_{1}=0$ and $s_{1}=s_{2}$, then the optimal amount of Asset 2 to hold is 0 . However, when $q_{1}>0$, then the optimal amount of Asset 2 to hold is less than 0. This highlights the behaviour that the investor tries to partially hedge a long position in one asset by acquiring a short position in the other (as well as try to reduce the long position in Asset 1 as well).

The intersection point of the corresponding attraction curves for $q_{1}$ and $q_{2}$ then acts as the attraction point for the vector-valued inventory process $\mathbf{q}_{t}$. Figure 4.6 is a graphical depiction of this attraction point as a function of $s_{1}-s_{2}$. 


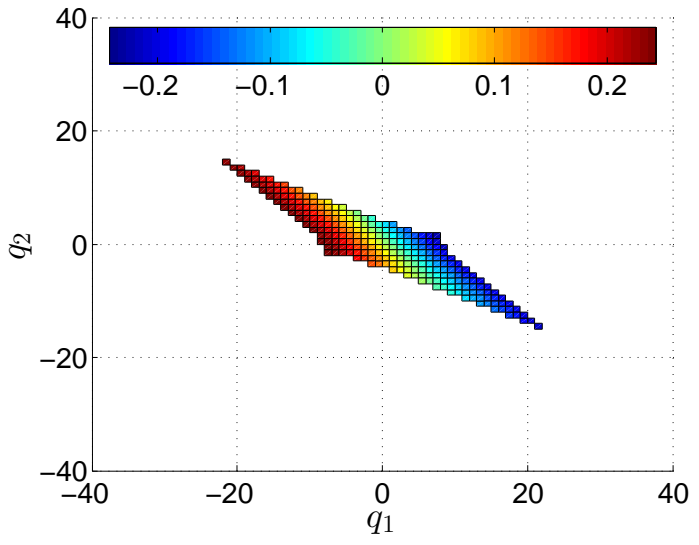

(a) Asset 1

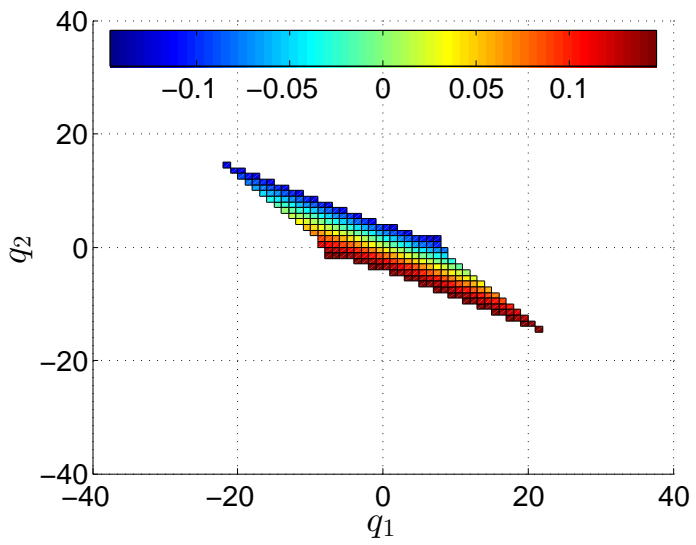

(b) Asset 2

Figure 4.4: Inventory drift when the assets' midprices are equal (i.e., $s_{1}=s_{2}$ ).

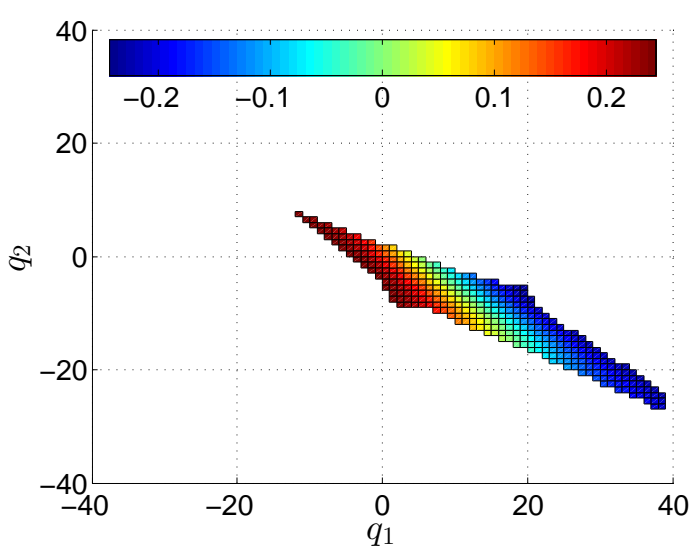

(a) Asset 1

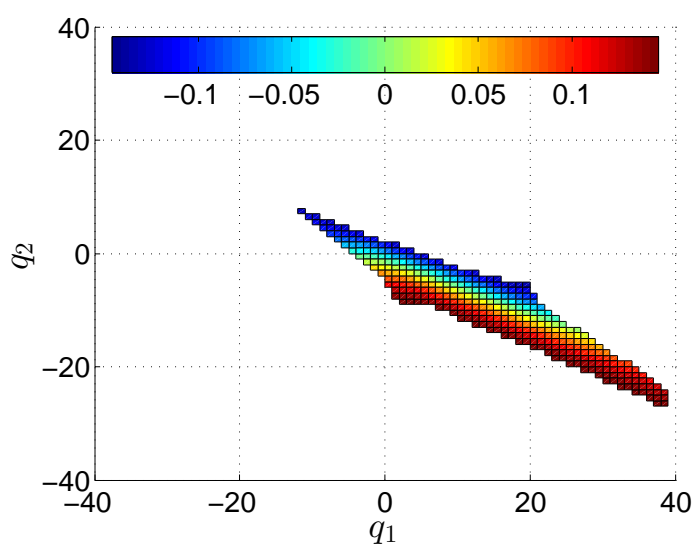

(b) Asset 2

Figure 4.5: Inventory drift when Asset 1 is cheap relative to Asset 2 (i.e., $s_{1}=s_{2}-0.012$ ). 


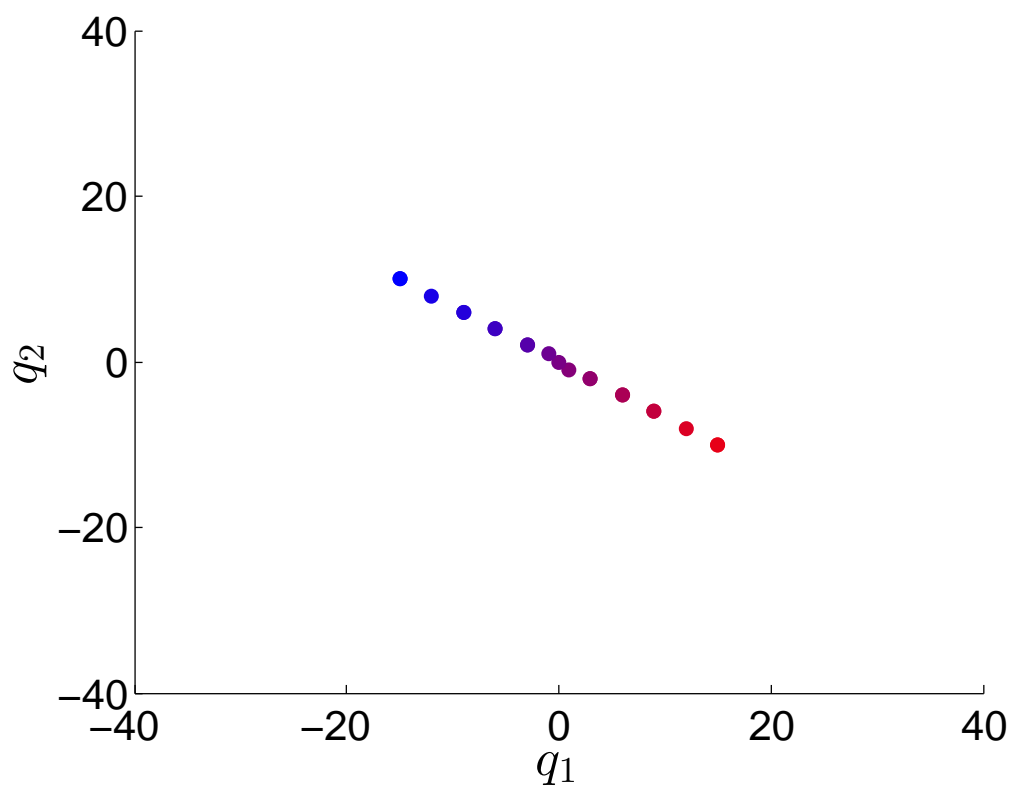

Figure 4.6: Mean reversion point of $\mathbf{q}$ as a function of $s_{1}-s_{2}$. The red (blue) dots correspond to small (large) values of $s_{1}-s_{2}$. 


\begin{tabular}{cc}
\hline \hline Parameter & Value \\
\hline$T$ & 300 \\
$\phi$ & $0.3,1,3,10,30,100,200$ \\
$\boldsymbol{\sigma}$ & $\left(\begin{array}{cc}0.005 & 0.009 \\
0 & 0.015\end{array}\right)$ \\
$\gamma$ & 0.001 \\
$\xi_{1}=\xi_{2}$ & 0.02 \\
$\lambda_{1}^{-}=\lambda_{1}^{+}$ & 1.2 \\
$\lambda_{2}^{-}=\lambda_{2}^{+}$ & 1.0 \\
$\kappa_{1}^{-}=\kappa_{1}^{+}$ & 40 \\
$\kappa_{2}^{-}=\kappa_{2}^{+}$ & 25 \\
$\boldsymbol{\zeta}$ & $\left(\begin{array}{cc}2 & 0 \\
0 & 2\end{array}\right)$ \\
$\rho$ & $0.40,0.50,0.60$ \\
$\epsilon$ & $0,0.01,0.02,0.04$ \\
\hline \hline
\end{tabular}

Table 4.2: Model parameters used in the simulation study. Recall that the fill rate assumption $h_{i}^{ \pm}(\delta)=\lambda_{i}^{ \pm} e^{-\kappa_{i}^{ \pm}\left(\delta-\xi_{i}\right)}$ from Example 4.3.3 is being made.

\subsection{Simulation Study: PnL of the Strategy}

In this section, we use simulations to analyze the financial performance of an investor who follows the optimal strategy previously discussed. We use the time limiting strategy for $T-t$ large since the convergence to the limiting strategy occurs quite quickly (typically within 15-30 seconds). The investor's operating frequency is every 5 milliseconds, the maximum amount of inventory the investor can hold long or short ${ }^{45}$ is $Q=40$ for each asset, and the trading horizon is $T=300$ seconds. Other model parameters are provided in Table 4.2. Recall that the assumption $h_{i}^{ \pm}(\delta)=\lambda_{i}^{ \pm} e^{-\kappa_{i}^{ \pm}\left(\delta-\xi_{i}\right)}$ from Example 4.3.3 is being made.

\subsubsection{Accounting for Adverse Selection}

As outlined in Section 3.4, to not include adverse selection effects in the data generating process of our simulation study would be to ignore a fundamental and prominent feature

\footnotetext{
${ }^{45}$ This bound on the investor's inventory only plays a role in the numerical solution of the value function. Depending on the penalty parameter $\phi>0$, the exact strategy will dictate uniform (over time and $\Omega$ ) bounds on the investor's inventory since the continuation region is bounded, as argued in Appendix C.6. This is visually depicted in Figure 4.2 where the optimal strategy keeps inventory inside the rhombus shaped region.
} 
of market microstructure. Hence, to analyze the strategy under more realistic scenarios, we assume that an important source of risk faced by the investor is adverse selection. The investor might be trading with better informed counterparties and therefore faces the risk of buying assets right before prices drop or selling assets right before prices rise. In our analysis, we include adverse selection by assuming that some MOs will affect the midprice of the asset.

With this in mind, we suppose that a MO originates from one of two sources. It was either executed by an informed trader, or an uninformed one. We assume that the probability that any particular $\mathrm{MO}$ comes from an informed trader is constant, and we shall denote this probability by $\rho$. We describe the two possible effects by example. Suppose the current best bid-offer (BBO) quotes for Assets 1 and 2 are 100.00/100.02 and 100.01/100.03, respectively. A large buy MO on Asset 1 arrives, widening the spread to $100.00 / 100.06$. At this point, one of two things will happen immediately:

- The MO was executed by an informed trader: The BBO quotes for Asset 1 becomes 100.04/100.06, giving an immediate impact of $\$ 0.04$. This impacts Asset 2 in the same way causing the BBO to move to $100.05 / 100.07$. The informed trader knows that there will be upward pressure on the price, and some other informed market participants (on both assets) move their current quotes to reflect this.

- The MO was executed by an uninformed trader: The BBO quotes for both assets remains unchanged. There is nothing special about this MO, and the depleted portion of the LOB refills with quotes.

This describes the immediate effect on the midprice process and made explicit in the SDE in (4.14).$^{46}$ Before we turn our attention to the persistent effect, some notation is in order. We define the vector-valued processes $\left\{\widehat{\mathbf{N}}_{t}^{-}\right\}_{0 \leq t \leq T}$ and $\left\{\widehat{\mathbf{N}}_{t}^{+}\right\}_{0 \leq t \leq T}$ to be the total number of informed market sell and buy orders up to time $t$, respectively, with the $i^{\text {th }}$ component representing Asset $i$. The persistent impact of an informed MO will come via a change in drift of the midprice process, $\mathbf{S}_{t}$. For each asset, we also have the following dynamics for the midprice process $\mathbf{S}_{t}$ :

$$
\left\{\begin{array}{l}
d \mathbf{S}_{t}=\boldsymbol{\alpha}_{t} d t+\boldsymbol{\sigma} d \mathbf{B}_{t}+\mathfrak{d}_{\left(N_{t-}+1\right)}\left(\begin{array}{cc}
1 & 1 \\
1 & 1
\end{array}\right) d\left(\widehat{\mathbf{N}}_{t}^{+}+\widehat{\mathbf{N}}_{t}^{-}\right) \\
d \boldsymbol{\alpha}_{t}=-\boldsymbol{\zeta} \boldsymbol{\alpha}_{t} d t+\mathfrak{e}_{\left(N_{t-}+1\right)} \Psi d\left(\widehat{\mathbf{N}}_{t}^{+}-\widehat{\mathbf{N}}_{t}^{-}\right)
\end{array}\right.
$$

where $\mathbf{S}_{t}$ has (normal) reflection at the boundary of $\mathcal{A}, \boldsymbol{\zeta}$ and $\Psi$ are $n \times n$ matricies with

\footnotetext{
${ }^{46}$ Since all of these effects occur simultaneously (or faster than the HFT can respond), this model does not admit latency arbitrage.
} 
non-negative entries, $\mathfrak{d}_{(1)}, \mathfrak{d}_{(2)}, \ldots$ is the sequence of random variables representing immediate market impact (will be non-negative for market buy orders and non-positive for market sell orders), and $\mathfrak{e}_{(1)}, \mathfrak{e}_{(2)}, \ldots$ is an iid sequence of exponential random variables with mean 1 . Note that because the immediate market impact term adds a random multiple of $(1,1)^{\prime}$ to $\mathbf{S}_{t}$, the resulting value of the midprice vector is guaranteed to remain inside the no-arbitrage region $\mathcal{A}$ (recall from Section 4.2 that $\mathcal{A}=\left\{\mathbf{s} \in \mathbb{R}^{2}:\left|s_{1}-s_{2}\right|<\frac{1}{2}\left(\xi_{1}+\xi_{2}\right)\right\}$ ) and $\alpha_{t}^{1}=\alpha_{t}^{2}$ (which will be denoted $\alpha_{t}$ ). Equation (4.14) is the natural multivariate extension of the drift dynamics presented in Cartea et al. (2014).

When an informed buy $\mathrm{MO}$ arrives on Asset $i$, then there will immediately be a jump in $\boldsymbol{\alpha}_{t}$ equal to the $i^{\text {th }}$ column of $\Psi$ multiplied by a random expontial random variable. The matrix $\boldsymbol{\zeta}$ serves as the decay matrix which forces each component of the process $\boldsymbol{\alpha}_{t}$ to mean revert to zero. The effect of an informed sell MO is similar, except it induces a negative jump in $\boldsymbol{\alpha}_{t}$.

Observe that we are using $d \widehat{\mathbf{N}}_{t}^{ \pm}$instead of $d \mathbf{N}_{t}^{ \pm}$or $d \overline{\mathbf{N}}_{t}^{ \pm}$in (4.14). This has the interpretation that only the MOs of informed traders will have an impact on $\boldsymbol{\alpha}_{t}$. Furthermore, our own market orders do not move the market, unlike the informed traders' MOs, as our MOs are quite small in size and we are a small player without superior market information. For the purposes of illustration, we make the further assumption that $\Psi=\epsilon\left(\begin{array}{ll}1 & 1 \\ 1 & 1\end{array}\right)$, where $\epsilon \geq 0$ is a scale parameter.

Figure 4.7 shows sample paths for the midprice process $\mathbf{S}_{t}$ and its drift process $\boldsymbol{\alpha}_{t}$. Observe the jumps in both the midprice process and the short term drift due to adverse selection. On this particular time window, Asset 1 is priced lower than Asset 2. Observe in Figure 4.7(c) how the midprice spends most of the time near the upper boundary. It will be shown in Section 4.5.1 that $g$ is only a function of $\mathbf{s}$ through the function $s_{1}-s_{2}$. We provide a sample path of this process in Figure 4.7(d). Again, notice that on this particular window $S_{t}^{(1)}-S_{t}^{(2)}<0$, but still obeying the bound $S_{t}^{(1)}-S_{t}^{(2)}>-\frac{1}{2}\left(\xi_{1}+\xi_{2}\right)=$ -0.02 .

Notice how the investor's buy LO quote for Asset 1 (as depicted in Figure 4.8(a)) varies as the process $S_{t}^{(1)}-S_{t}^{(2)}$ varies over time in Figure 4.7(d). The discrete jumps in $\delta_{1, t}^{-}$ are due to the state variable $s_{1}-s_{2}$ moving to another (discrete) grid point (recall that $g(t, \mathbf{s}, \mathbf{q})$ was solved numerically on a sparse grid for the spatial variable $\mathbf{s})$. In this plot, the cheaper Asset 1 is relative to Asset 2 (i.e. when $S_{t}^{(1)}-S_{t}^{(2)}$ is close to -0.02), the more willing the investor is to acquire an additional unit of Asset 1. Hence, she posts her limit buy quote closer to the best bid for Asset 1 and her limit sell quote closer to the best offer for Asset 2. Along this sample path, the investor managed to acquire a long position in Asset 1, as shown in 4.8(b), and is using her short position in Asset 2 to 


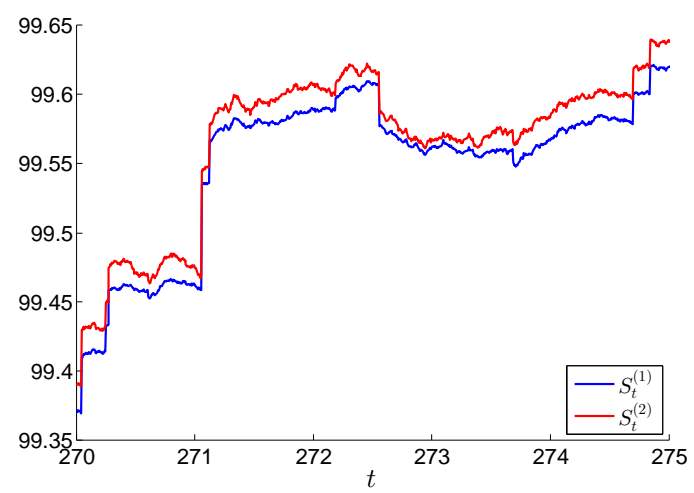

(a) Midprice Process

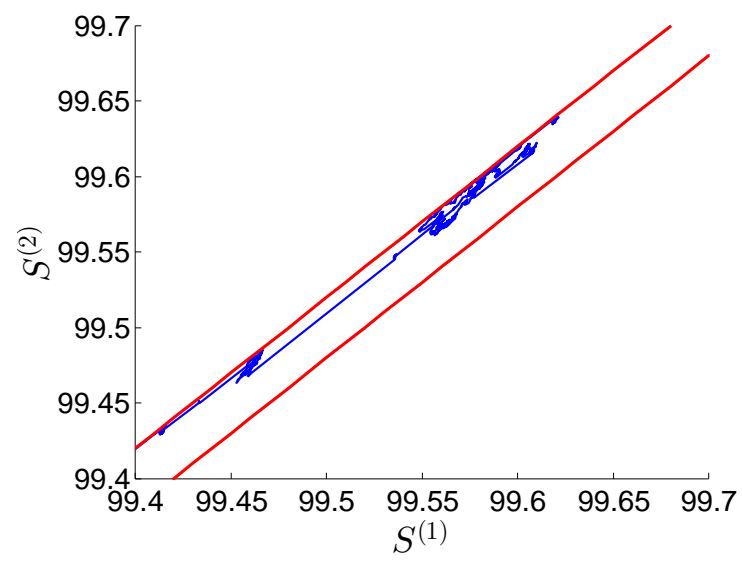

(c) Midprice Process

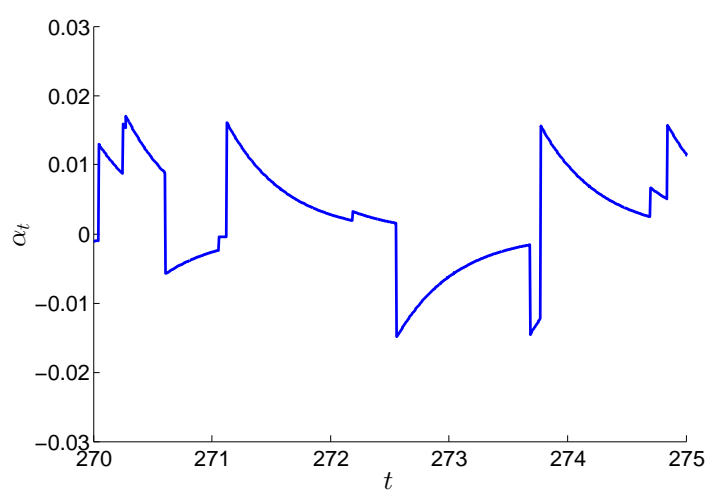

(b) Drift Process, $\alpha_{t}=\alpha_{t}^{1}=\alpha_{t}^{2}$

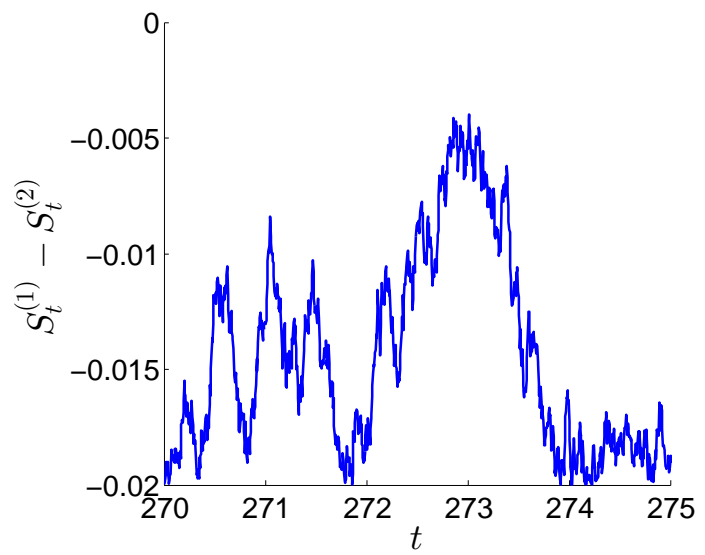

(d) Dimensionally Reduced Midprice Process

Figure 4.7: Sample paths of the midprice process $\mathbf{S}_{t}$ and drift process $\boldsymbol{\alpha}_{t}$. The red lines in Figure 4.7(c) represent the boundary of the no-arbitrage region.

partially hedge away the risk associated with being long the underlying equity.

Although these adverse selection effects are present in the DGP, the trader cannot estimate the correct drift of the asset so she assumes that it is zero. Recall that the investor assumes that the dynamics of $\mathbf{S}_{t}$ are given by Assumption 4.2.4, and that this was the assumption used when solving the stochastic control problem in Section 4.3.1.

\subsubsection{Results of the Simulation Study}

Here we show how the investor's strategy performs when we vary the running inventory penalty $\phi$ and her exposure to adverse selection by varying the scale parameter $\epsilon$. The investor starts with zero inventory in both assets and will trade for $T=300$ seconds. We 


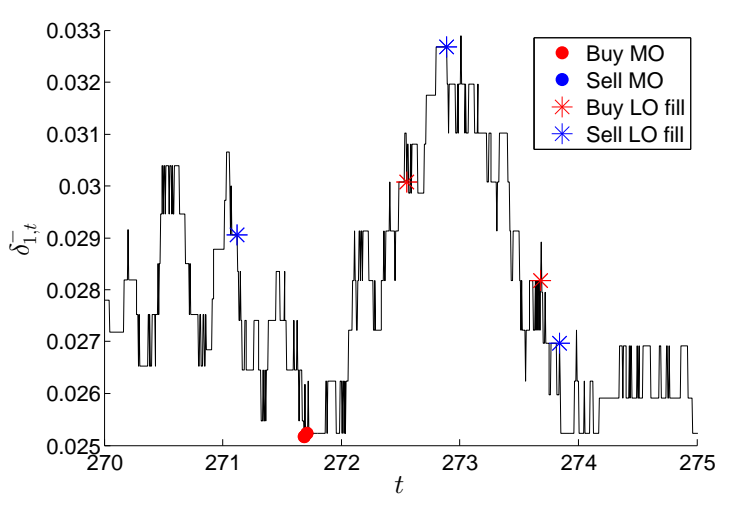

(a) Optimal Asset 1 Buy LO Depth

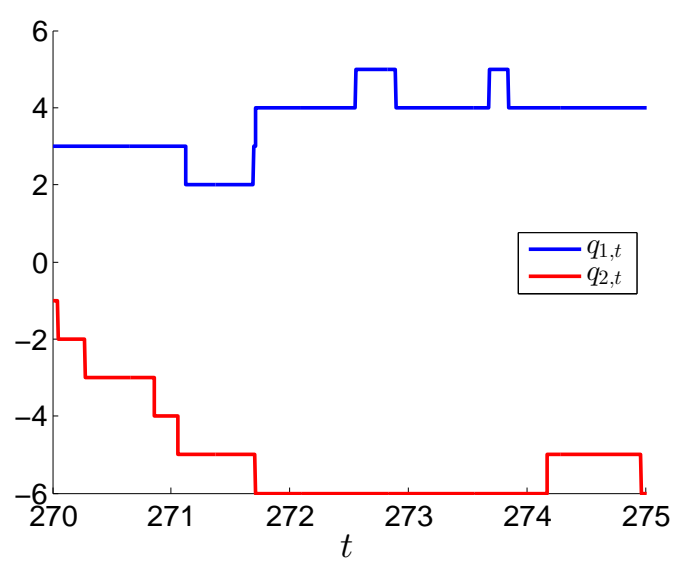

(b) Inventory Process

Figure 4.8: Sample paths of the investor's LO depth and her inventory level for $\phi=10$.

run 2,500 simulations and record the PnL for each run. Figure 4.9 provides histograms depicting the distribution of the terminal PnL for two levels of running inventory penalty. In Figure 4.9(a) we assume that the investor is not exposed to adverse selection costs by assuming $\epsilon=0$ and in Figure 4.9(b) we show the histogram of the PnLs when the investor is exposed to adverse selection effects by setting $\epsilon=0.02$.

However, when we increase the scale parameter $\epsilon$ in the jump size of the short term drift process, we can observe that the less inventory averse investor does not necessarily outperform her more inventory averse counterpart. This is due to the behaviour that the less inventory averse investor is willing to hold larger inventories for a longer period of time which further exposes her to the drift of $\mathbf{S}_{t}$, while the more inventory averse investor cuts her losses early in an attempt to reduce inventory risk. The $\epsilon=0.04$ case in Figure 4.10 illustrates this result very well.

There is some debate about which risk metric better characterize the riskiness of the strategy. Classical work in finance is typically only concerned with the terminal distribution of PnL (at time T); namely, its variance (or standard deviation) and possibly higher moments that give information about the weight of the tails. However, considering the penalty term in (4.3), we see that we are concerned with the optimal portfolio's risk throughout the entire time interval $[0, T]$, not just at the end. To this end, we classify a strategy's riskiness via the penalty $\int_{0}^{T} \ell\left(\mathbf{q}_{u}\right) d u$ and consider the mean of this quantity. This is what is termed mean inventory risk.

Figures 4.10 and 4.11 provide efficient frontiers comparing expected PnL against both standard deviation of $\mathrm{PnL}$ and mean inventory risk for a variety of adverse selection parameters ( $\epsilon$ and $\rho$, respectively). Decreasing $\phi$ (i.e. increasing risk appetite) corresponds 


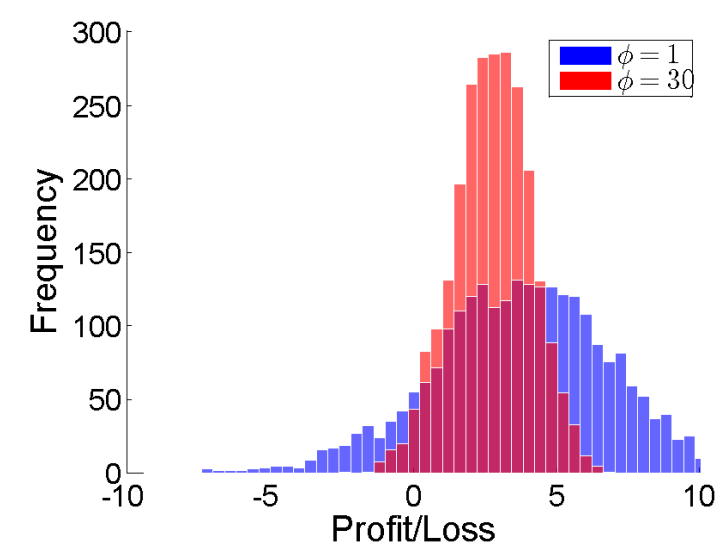

(a) $\epsilon=0$

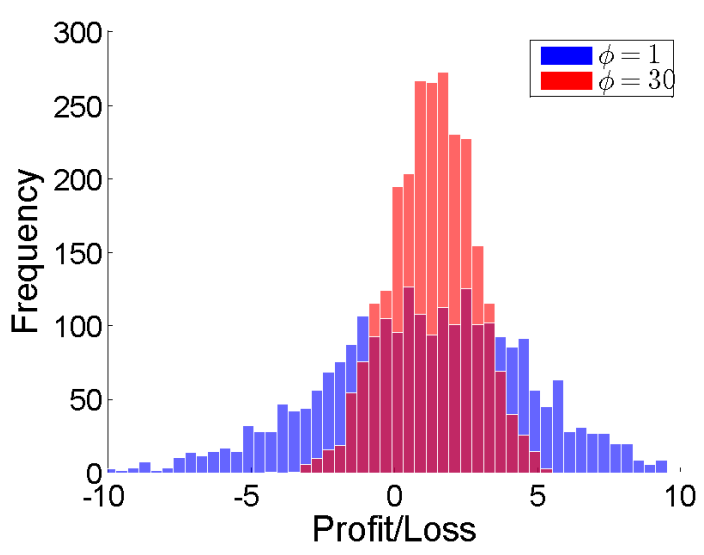

(b) $\epsilon=0.02$

Figure 4.9: Distribution of terminal profit and loss. The probability of an informed MO is fixed at $\rho=0.50$.

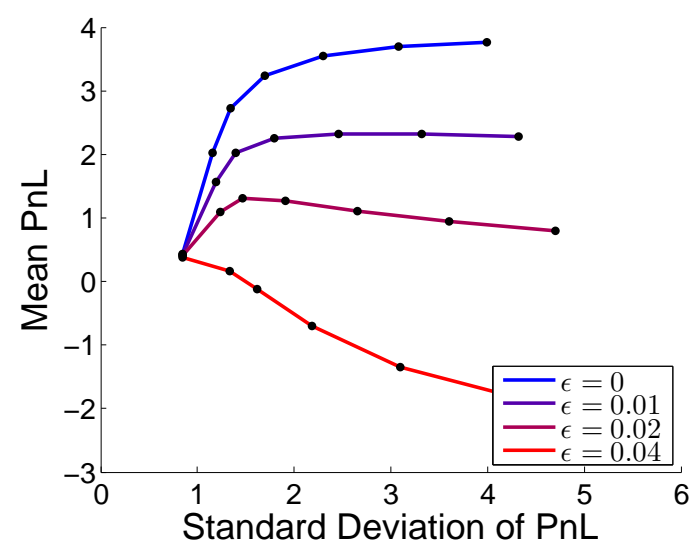

(a) Mean PnL vs Std Dev PnL

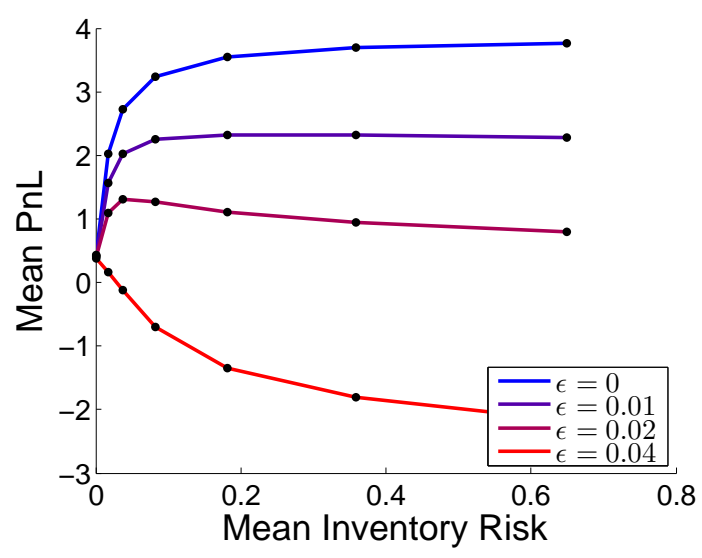

(b) Mean PnL vs Mean Inventory Risk

Figure 4.10: Performance of strategy for various $\phi$ with different drift impact scale parameters $\epsilon$. The probability of an informed MO is fixed at $\rho=0.50$.

to moving in the rightward direction of this plot. As stated in Table 4.2, $\phi$ ranges from 0.3 to 200 .

Recall that the investor assumes that the drift of $\mathbf{S}_{t}$ is zero, when in fact there are adverse selection effects. As outlined in detail in Section 4.4.1, we have a scale parameter $\epsilon \geq 0$ that represents the magnitude of such effects. The larger the parameter, the stronger the effects of adverse selection are in the market. It is clear from Figure 4.10, that adverse selection generally has a negative effect on the investor's PnL, which is consistent with the results in Section 3.6 for the market maker that cannot detect influential MOs. In the extreme case of $\epsilon=0.04$, these effects are so strong that decreasing the investor's 


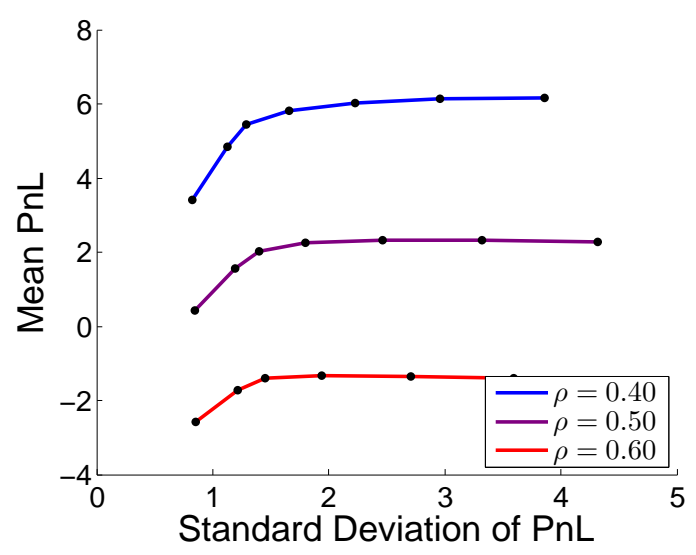

(a) Mean PnL vs Std Dev PnL

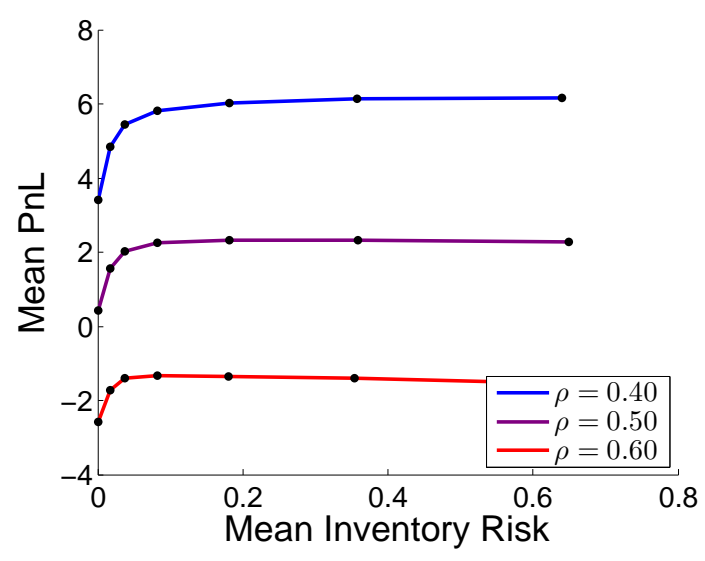

(b) Mean PnL vs Mean Inventory Risk

Figure 4.11: Performance of strategy for various $\phi$ with different probabilities of informed MOs $\rho$. The drift impact scale parameter is fixed at $\epsilon=0.01$.

inventory aversion actually decreases return because she is allowing her portfolio to be exposed to these effects for a longer period of time.

Up until this point, we have assumed that $50 \%$ of all incoming MOs are influential and thus have an impact on the drift of the midprice. Recall that MOs of this type have two effects: an immediate effect and a persistent effect. If we increase the probability $\rho$ that an $\mathrm{MO}$ comes from an informed trader, then this will directly translate into an increase in the number of MOs that immediately move the market. Because this movement is always against the investor when her LO is filled, it will always have a negative impact on her PnL (in contrast to increasing $\epsilon$ where this was not the case). The resulting efficient frontiers are reported in Figure 4.11 for three differing values of $\rho$.

We observe in Figure 4.12 that the larger the inventory penalty parameter $\phi$ is, the more MOs are executed by the trader. ${ }^{47}$ We obtain this result because larger $\phi$ means that the trader imposes a higher penalty on running inventory, which induces higher mean reversion in inventories. This is attained by adjusting the LOs accordingly and executing more market orders. Alternatively, one can also see that the larger $\phi$ is, the smaller the continuation region, so the boundary gets breached more often inducing MOs that force the investor to cross the bid-ask spread to bring $\mathbf{q}$ back into the continuation region.

Although there is symmetry between the buy and sell sides of the LOB, there is an intuitive explanation for the drastic difference in number of executed MOs between the

\footnotetext{
${ }^{47}$ It was brought to the author's attention that just submitting MOs is counter-intuitive to the notion of market marking. To clarify, LOs are still an integral part of this investor's strategy. Moreover, if the control problem is solved with just the execution of MOs, then the optimal strategy is to do nothing.
} 


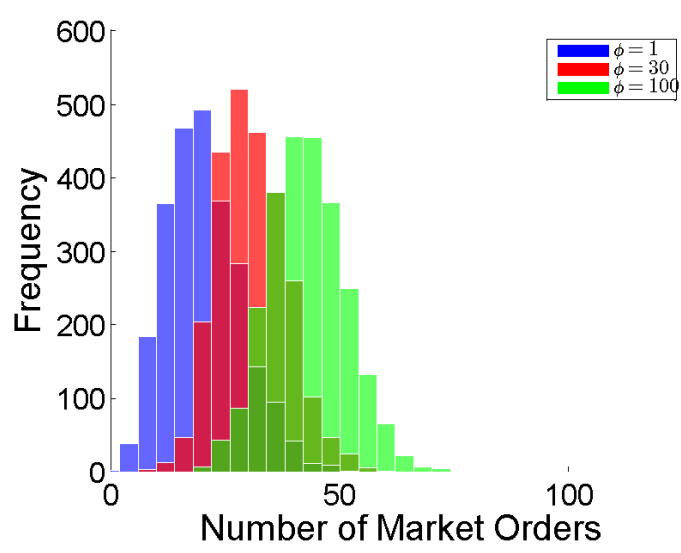

(a) Asset 1 - Market Buy

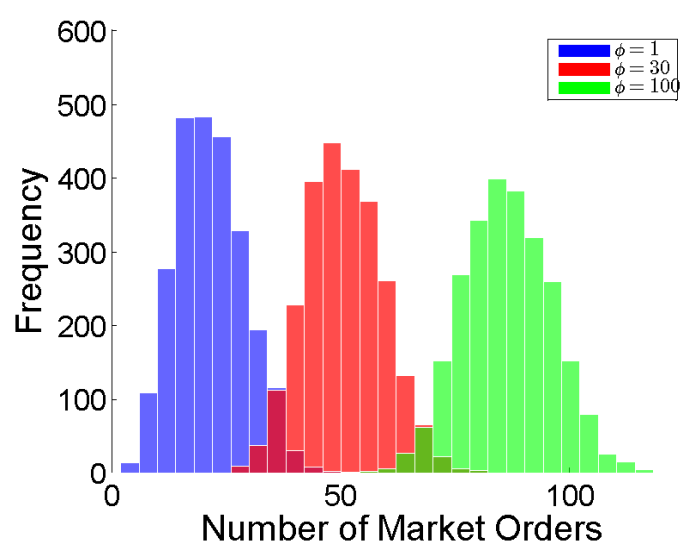

(c) Asset 2 - Market Buy

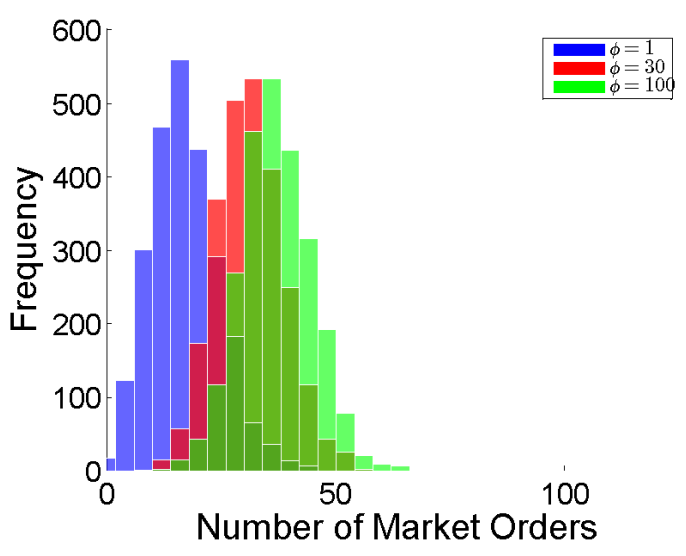

(b) Asset 1 - Market Sell

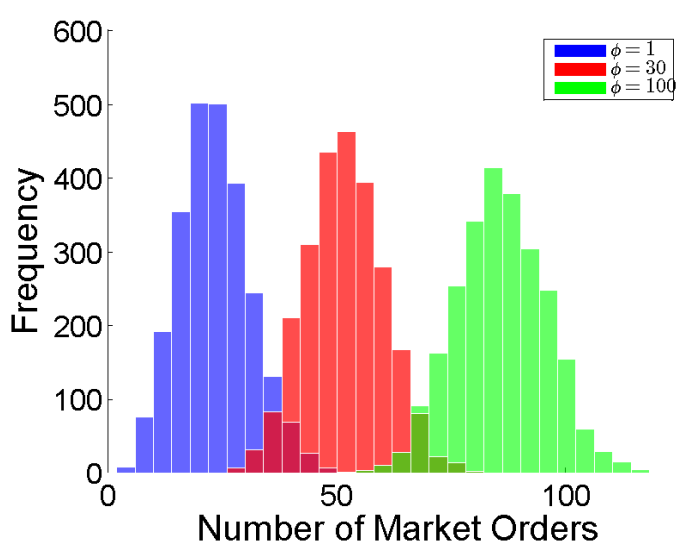

(d) Asset 2 - Market Sell

Figure 4.12: Distribution of the number of executed MOs for various inventory penalty levels when $\epsilon=0.01$ and $\rho=0.50$.

two assets. This is simply due to the fact that Asset 2 has a higher total volatility, which increases the penalty for holding a position in it. Hence, the continuation region is smaller in the $q_{2}$ direction than in the $q_{1}$ direction (as seen in Figure 4.2).

\subsubsection{Comparison to Other Strategies}

As mentioned previously, the simplified economy consisting of correlated assets without being over-complete is a special case of the framework that is presented in this chapter. There are many simplifications in this case, a particularly important simplification is that, in this case, $g$ would be independent of $\mathbf{s}$ (i.e. $\operatorname{dim} B=0$ ) since the no-arbitrage region is all of $\mathbb{R}^{n}$. Such an economy does not admit a non-zero vector $\mathbf{b}$ and time $\bar{T}$ 


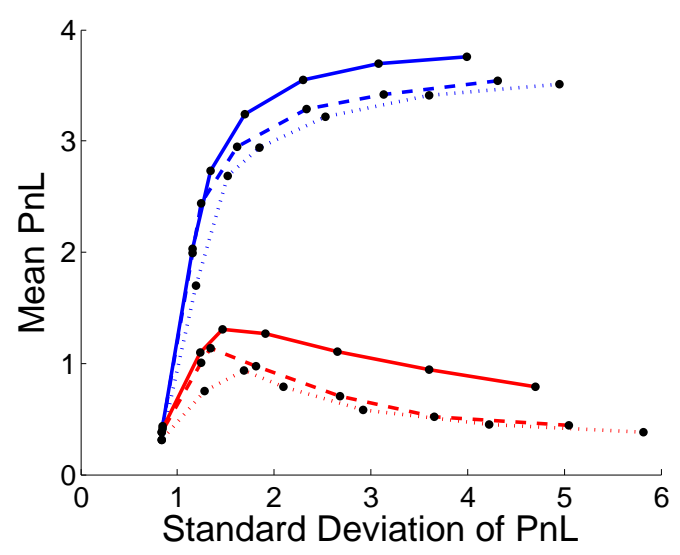

(a) Mean PnL vs Std Dev PnL

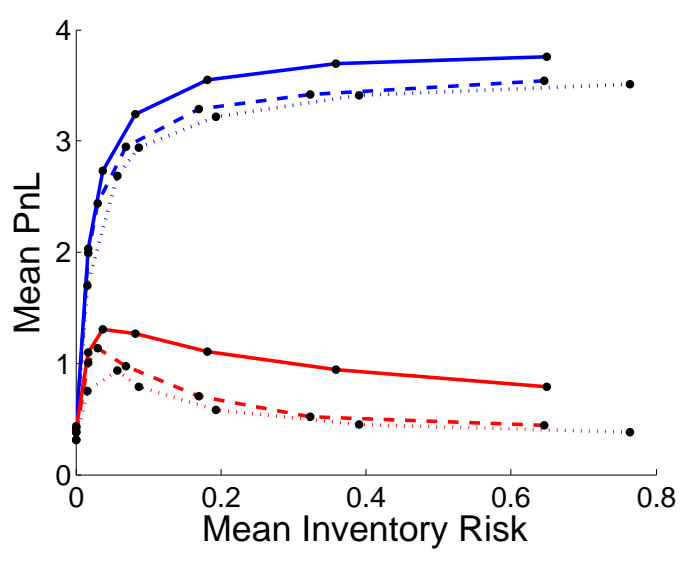

(b) Mean PnL vs Mean Inventory Risk

Figure 4.13: Comparison of efficient frontiers for the full strategy (solid lines), the no boundary strategy (dashed line), and the single asset benchmark (dotted line). The blue curves refer to $\epsilon=0$ and the red curves refer to $\epsilon=0.02$. In both cases, $\rho=0.50$.

such that $\mathbb{V}\left[\mathbf{b} \cdot \mathbf{S}_{\bar{T}}\right]=0$. In what follows, we investigate the PnL of the investor who trades as if the assets are correlated, but ignores the no-arbitrage boundaries. However, the DGP will be one in which the assets do indeed reflect into the no-arbitrage region. We call this the no boundary case.

A second alternative strategy is that of an investor who makes markets in each asset individually and is ignorant of the co-dependence between the assets through their correlation and the reflection into the no-arbitrage region. That is, she treats each asset as its own isolated 1-asset economy. This strategy is referred to as the single asset benchmark and is exactly the strategy of Section 2.4.

Figure 4.13 compares the multi-asset strategy, with and without considering reflection at the boundaries, as well as the single asset strategies. Observe the marginal gain in the no boundary case over the single asset benchmark. This illustrates the value in using the correlation between the assets to offset some of the inventory risk, in particular for medium to large values of $\phi$.

More prominent in Figure 4.13 is the effect on PnL due to the investor considering the no-arbitrage boundary and adjusting her LO quotes accordingly. Observe the gain in mean PnL (for the same level of risk) for the full strategy over the no boundary strategy. This supports the conclusion that there is value in the consideration of the no-arbitrage region in over-complete markets. 


\subsection{Numerical Scheme for Exponential Fill Rate}

This section outlines the technical details regarding the existence of a unique viscosity solution (see Appendix B.2) to the dynamic programming equation (DPE), specifies a dimensional reduction in the spacial variables, gives explicit details regarding the discrete numerical scheme, and proves that the discretized value function converges to the unique viscosity solution.

\subsubsection{Dimensional Reduction of Spatial Variables}

We can use the following lemma to gain a dimensional reduction in the spatial variable $\mathbf{s}$. Also notice that due to this lemma, the relevant $\mathbf{s}$ values now live in a compact domain. For a fixed error, the total run time will then be proportional to $c^{\operatorname{dim} B}(2 Q+1)^{n}$, where $c$ is some constant that depends on the spreads $\xi_{1}, \xi_{2}, \ldots, \xi_{n}$, the basis vectors that span $B$ (or equivalently on the number of discrete steps on the dimensionally reduced s-grid), and $Q$ is the maximum inventory the investor can be long or short for each asset.

Lemma 4.5.1. There exists a $C^{2}$, surjective function $\Gamma: \overline{\mathcal{A}} \longrightarrow \overline{\mathcal{A}}^{\prime} \subset \mathbb{R}^{n^{\prime}}$ such that:

(I) For all $\mathbf{s} \in \overline{\mathcal{A}}, g(\mathbf{s}, \cdot)=g((\Gamma(\mathbf{s}), \mathbf{0}), \cdot)$.

(II) $\mathbf{s} \in \partial \mathcal{A} \quad \Longleftrightarrow \quad \Gamma(\mathbf{s}) \in \partial \mathcal{A}^{\prime}$.

(III) $\overline{\mathcal{A}}^{\prime}$ is the cartesian product of closed, bounded intervals.

(IV) $\operatorname{dim} \overline{\mathcal{A}}^{\prime}=\operatorname{dim} B=n^{\prime}$.

Proof. See Appendix C.5.

Lemma 4.5.1 states that it is not necessary to know $\mathbf{s}$ to get the value of $g(\mathbf{s}, \cdot)$; we only require the information contained in $\Gamma(\mathbf{s})$. Furthermore, there exists such a function $\Gamma$ that provides a nice, smooth mapping between the original $n$-dimensional no-arbitrage region $\overline{\mathcal{A}}$ and the new $n^{\prime}$-dimensional no-arbitrage region $\overline{\mathcal{A}}^{\prime}$, and that this new region is a bounded rectangle. The fact that the new region is bounded improves the accuracy of the numerical scheme in Section 4.5.2 since we no longer have to approximate the unbounded set $\overline{\mathcal{A}}$ by a bounded one.

There is, however, a minor technical detail that has been overlooked. The form $(\Gamma(\mathbf{s}), \mathbf{0})$, where $\mathbf{0}$ is an $\left(n-n^{\prime}\right)$-dimensional vector of 0 's, requires the assets to be ordered in a 
certain way. In an arbitrary ordering, the components of $(\Gamma(\mathbf{s}), \mathbf{0})$ will need to be reordered. We continue with the labels that produce a simplification of this form as it is more compact to write $(\Gamma(\mathbf{s}), \mathbf{0})$ instead of having the 0 's in arbitrary positions (i.e. not consecutive nor all at the beginning or end).

In some sense, $\Gamma(\mathbf{s})$ is sufficient $t^{48}$ for $g$. That is, $\Gamma(\mathbf{s})$ contains the same amount of information as $\mathbf{s}$ when computing $g$. Furthermore, one could say that $\Gamma(\mathbf{s})$ is minimal sufficient since it can be written as a function of any other function of $\mathbf{s}$ that is sufficient for $g$. Stated heuristically, $\Gamma(\mathbf{s})$ contains the smallest amount of information about $\mathbf{s}$ that still allows us to know $g(\mathbf{s})$ with certainty. The construction of $\Gamma$ in the proof of Lemma 4.5.1 is used in what follows to obtain a dimensional reduction, and is given by

$$
\Gamma(\mathbf{s})=\left[b_{j} \cdot \mathbf{s}\right]_{j} .
$$

In our 2-dimensional illustrative example, we take $\Gamma(\mathbf{s})=s_{1}-s_{2}$. For the 3 -dimensional example with two nearby futures and the 1-2 calendar spread, one could take $\Gamma(\mathbf{s})=$ $s_{1}-s_{2}-s_{3}$. More details for these two examples are provided in Appendix E.3.

The following Corollary states that the function $g$ is invariant to movements in the direction of any element of $B^{\perp}$. This would correspond to vectors of the form $(1,1)$ in our illustrative example (i.e. $g$ is remains unchanged when the futures and its underlying both increase or decrease by exactly the same amount).

Corollary 4.5.2. $D_{v} g=0$ on $\overline{\mathcal{A}}$ whenever $v \in B^{\perp}$.

Proof. Let $\mathbf{s} \in \overline{\mathcal{A}}$ and $v \in B^{\perp}$. Note that we necessarily have $\mathbf{s}+v \in \overline{\mathcal{A}}$ by Equation (C.6) since $b_{i} \cdot v=0$ for $i=1, \ldots, n^{\prime}$. We then have $\Gamma(\mathbf{s})=\Gamma(\mathbf{s}+v)$ by (4.15). But $v \in B^{\perp}$ is arbitrary and $B^{\perp}$ is a subspace, so one can consider a limiting sequence of the form $\left\{\eta_{k} v\right\}$, with the scalar sequence $\eta_{k} \downarrow 0$, which yields $D_{v} g=0$.

\subsubsection{Description of Numerical Scheme}

We numerically solve (4.12) by applying upper and lower bounds to each component in $\mathbf{q}$, and solving the corresponding ODEs backwards in time using an explicit numerical scheme. For any fixed $\mathbf{q}$, we have an $n$-dimensional discrete grid to solve for various

\footnotetext{
${ }^{48}$ In mathematical statistics, a statistic $U$ is said to be sufficient for an unknown model parameter $\Theta$ if the conditional distribution of the data, conditional on $U$, is independent of $\Theta$. Heuristically speaking, $U$ contains the same amount of information about $\Theta$ as the data itself.
} 
values of $\mathbf{s}$. Once we have this grid for each $\mathbf{q}$, we propagate one step backwards in $t$ and repeat the process.

Define the domain for $\mathbf{s}, \mathcal{A}^{\prime} \subset \mathbb{R}^{n^{\prime}}$, on the dimensionally reduced grid. Let $\mathbb{T}_{d t}$ be a regular partition of $[0, T], \mathbb{S}_{d s}$ be an $n^{\prime}$-dimensional regular rectangular partition of the dimensionally reduced s-grid $\overline{\mathcal{A}}^{\prime}$, and $\mathbb{Y}_{Q}$ be the subset of $\mathbb{Z}^{n}$ that corresponds to inventory with maximum $Q$ as previously described (i.e. $\mathbb{Y}_{Q}=\mathbb{Z}^{n} \cap[-Q, Q]^{n}$ ). Throughout the rest of this chapter, $d t>0$ and $d s>0$ refer to the discrete step sizes in the time $(t)$ and space $(\Gamma(\mathbf{s}))$ variables, repsectively.

Define, for any function $\varphi:[0, T] \times \overline{\mathcal{A}}^{\prime} \times \mathbb{Z}^{n} \longrightarrow \mathbb{R}$, the discrete dynamic evolution operator

$$
\mathcal{S}^{d t, d s, Q}(t, \mathbf{s}, \mathbf{q}, \varphi)=\max \left[\mathcal{T}^{d t, d s, Q}(t, \mathbf{s}, \mathbf{q}, \varphi), \mathcal{M}^{d t, d s, Q}(t, \mathbf{s}, \mathbf{q}, \varphi)\right]
$$

with the component corresponding to the continuation region given by

$$
\begin{aligned}
& \mathcal{T}^{d t, d s, Q}(t, \mathbf{s}, \mathbf{q}, \varphi)=\varphi+d t\left[\frac{1}{2} \sum_{i, j=1}^{n}\left[\boldsymbol{\sigma} \boldsymbol{\sigma}^{\prime}\right]_{i j} \widehat{\mathcal{T}}_{i j}^{d t, d s, Q}(\cdot, \varphi)+\sum_{i, \pm} \frac{\lambda}{\kappa e} \exp \left\{\frac{\kappa \xi}{2}+\kappa \Delta \varphi\right\}-\phi \ell(\mathbf{q})\right], \\
& \widehat{\mathcal{T}}_{i j}^{d t, d s, Q}(t, \mathbf{s}, \mathbf{q}, \varphi)=\frac{\varphi\left(\Gamma\left(s_{i}+d s, s_{j}+d s\right), \cdot\right)-\varphi\left(\Gamma\left(s_{i}+d s\right), \cdot\right)-\varphi\left(\Gamma\left(s_{j}+d s\right), \cdot\right)+\varphi(\cdot)}{d s^{2}},
\end{aligned}
$$

and the component corresponding to the impulse region given by

$$
\mathcal{M}^{d t, d s, Q}(t, \mathbf{s}, \mathbf{q}, \varphi)=\max _{i, \pm}\left\{\varphi\left(t, \mathbf{s}, \mathbf{q} \pm e_{i}\right)-\xi_{i} / 2\right\}
$$

We approximate the solution $g$ by $g^{d t, d s, Q}: \mathbb{T}_{d t} \times \mathbb{S}_{d s} \times \mathbb{Y}_{Q} \longrightarrow \mathbb{R}$ defined by:

$$
\left\{\begin{aligned}
g^{d t, d s, Q}(T, \mathbf{s}, \mathbf{q}) & =-\sum q_{i}\left(\operatorname{sign}\left(q_{i}\right) \frac{\xi_{i}}{2}+\gamma q_{i}\right) \\
g^{d t, d s, Q}(d t \cdot k, \mathbf{s}, \mathbf{q}) & =\mathcal{S}^{d t, d s, Q}\left(d t \cdot(k+1), \mathbf{s}, \mathbf{q}, g^{d t, d s, Q}\right)
\end{aligned}\right.
$$

We employ an explicit scheme backwards in time as follows:

- When $t=T$, for each $\mathbf{s}$ and $\mathbf{q}$ on their respective grids, set

$$
g^{d t, d s, Q}(T, \mathbf{s}, \mathbf{q})=-\sum q_{i}\left(\operatorname{sign}\left(q_{i}\right) \frac{\xi_{i}}{2}+\gamma q_{i}\right) .
$$

- For $t<T$ and $\mathbf{s} \in \mathcal{A}^{\prime}$, we perform the following: 
- Compute $\mathcal{T}^{d t, d s, Q}\left(t+d t, \mathbf{s}, \mathbf{q}, g^{d t, d s, Q}\right)$ and store the optimal control $\delta$. Some details for handling the mixed partial term are given in Appendix E.3.1.

- Compute $\mathcal{M}^{d t, d s, Q}\left(t+d t, \mathbf{s}, \mathbf{q}, g^{d t, d s, Q}\right)$ and store the optimal impulse $\tau$.

- If $\mathcal{T}^{d t, d s, Q}\left(t+d t, \mathbf{s}, \mathbf{q}, g^{d t, d s, Q}\right) \geq \mathcal{M}^{d t, d s, Q}\left(t+d t, \mathbf{s}, \mathbf{q}, g^{d t, d s, Q}\right)$, then set $g^{d t, d s, Q}(t, \mathbf{s}, \mathbf{q})=\mathcal{T}^{d t, d s, Q}\left(t+d t, \mathbf{s}, \mathbf{q}, g^{d t, d s, Q}\right)$. Otherwise, set $g^{d t, d s, Q}(t, \mathbf{s}, \mathbf{q})=\mathcal{M}^{d t, d s, Q}\left(t+d t, \mathbf{s}, \mathbf{q}, g^{d t, d s, Q}\right)$.

- For $t<T$ and $\mathbf{s} \in \partial \mathcal{A}^{\prime}$, we perform the following:

- Numerically approximate the condition $(\mathbf{q}+\nabla g) \cdot \boldsymbol{n}=0$, similar to what is done in Appendicies E.3.3 and E.3.4 for the $n=2$ and $n=3$ examples, respectively.

\subsubsection{Convergence of the Numerical Scheme}

We require the following bounds on the value function to ensure the existence of a unique viscosity solution to the DPE.

Lemma 4.5.3. There exists constants $c_{0}, c_{1}, c_{2} \in \mathbb{R}$ such that

$$
x+\sum_{i} q_{i}\left(s_{i}-\operatorname{sign}\left(q_{i}\right) \frac{\xi_{i}}{2}\right) \leq \Phi \leq x+\mathbf{q} \cdot \mathbf{s}+c_{0}(T-t)+c_{1}\|\mathbf{q}\|_{1}+c_{2} .
$$

Proof. See Appendix C.6.

Similar to Guilbaud and Pham (2013a), we first prove monotonicity, stability, and consistency properties (Propositions 4.5.4, 4.5.5, and 4.5.6, respectively) of the discrete dynamic evolution operator $\mathcal{S}^{d t, d s, Q}(t, \mathbf{s}, \mathbf{q}, \cdot)$. These properties are critical in the proof of Theorem 4.5.7, which states that the solution to the numerical scheme, as given by (4.16), converges locally uniformly to the unique viscosity solution $g(t, \mathbf{s}, \mathbf{q})$.

Proposition 4.5.4. (Monotonicity) For any $d s>0$, there exists $h_{t}>0$ (depends on ds) such that for any $0<d t<h_{t}, Q \in \mathbb{N}$, and $\varphi_{1}, \varphi_{2} \in C_{b}^{1,2}\left([0, T] \times \overline{\mathcal{A}}^{\prime} \times \mathbb{Z}^{n}\right)$ with $\varphi_{1} \leq \varphi_{2}$, we have $\mathcal{S}^{d t, d s, Q}\left(t, \mathbf{s}, \mathbf{q}, \varphi_{1}\right) \leq \mathcal{S}^{d t, d s, Q}\left(t, \mathbf{s}, \mathbf{q}, \varphi_{2}\right)$.

Proof. See Appendix C.7. 
Proposition 4.5.5. (Stability) For any $d t, d s>0$ and $Q \in \mathbb{N}$, there exists a unique solution $g^{d t, d s, Q}(t, \mathbf{s}, \mathbf{q})$ to (4.16). Furthermore, the sequence $\left\{g^{d t, d s, Q}\right\}$ is uniformly bounded. More formally, there exists $c_{0}, c_{1}, c_{2} \in \mathbb{R}$ such that for any $(t, \mathbf{s}, \mathbf{q}) \in \mathbb{T}_{d t} \times \mathbb{S}_{d s} \times \mathbb{Y}_{Q}$,

$$
-\sum_{i} q_{i} \operatorname{sign}\left(q_{i}\right) \frac{\xi_{i}}{2} \leq g^{d t, d s, Q}(t, \mathbf{s}, \mathbf{q}) \leq c_{0}(T-t)+c_{1}\|\mathbf{q}\|_{1}+c_{2} .
$$

Proof. Existence and uniqueness follows immediately from the definition of the explicit discretization scheme given in (4.16). The upper bound is a direct consequence of Lemma 4.5.3 since $g^{d t, d s, Q}(t, \mathbf{s}, \mathbf{q}) \leq g(t, \mathbf{s}, \mathbf{q})=\Phi-x-\mathbf{q} \cdot \mathbf{s} \leq c_{0}(T-t)+c_{1}\|\mathbf{q}\|_{1}+c_{2}$. The lower bound is attained by immediate liquidation of the current inventory and then doing nothing until $t=T$.

Proposition 4.5.6. (Consistency) For all $(t, \mathbf{s}, \mathbf{q}) \in[0, T) \times \overline{\mathcal{A}}^{\prime} \times \mathbb{Z}^{n}$ and $\varphi \in C_{b}^{1,2}\left([0, T] \times \overline{\mathcal{A}}^{\prime} \times \mathbb{Z}^{n}\right)$, we have

$$
\begin{aligned}
& \lim _{\begin{aligned}
(d t, d s, Q) \rightarrow(0,0, \infty) \\
\left(t^{\prime}, \mathbf{s}^{\prime}\right) \rightarrow(t, \mathbf{s})
\end{aligned}} \frac{1}{d t}\left[\mathcal{T}^{d t, d s, Q}\left(t^{\prime}+d t, \mathbf{s}^{\prime}, \mathbf{q}, \varphi\right)-\varphi\left(t^{\prime}, \mathbf{s}^{\prime}, \mathbf{q}\right)\right]= \\
& \quad \frac{\partial \varphi}{\partial t}+\frac{1}{2} \sum_{i, j=1}^{n}\left[\boldsymbol{\sigma} \boldsymbol{\sigma}^{\prime}\right]_{i j} \frac{\partial^{2} \varphi}{\partial s_{i} \partial s_{j}}+\sum_{i, \pm} \frac{\lambda}{\kappa} \exp \left\{-1+\frac{\kappa \xi}{2}+\kappa \Delta \varphi\right\}-\phi \ell(\mathbf{q})
\end{aligned}
$$

and

$$
\lim _{\substack{(d t, d s, Q) \rightarrow(0,0, \infty) \\\left(t^{\prime}, \mathbf{s}^{\prime}\right) \rightarrow(t, \mathbf{s})}} \mathcal{M}^{d t, d s, Q}\left(t^{\prime}+d t, \mathbf{s}^{\prime}, \mathbf{q}, \varphi\right)=\max _{i, \pm}\left\{\Delta_{i}^{ \pm} \varphi-\xi_{i} / 2\right\} .
$$

Proof. See Appendix C.8.

Theorem 4.5.7. (Convergence) The solution $g^{d t, d s, Q}$ to the numerical scheme (4.16) converges locally uniformly to the unique viscosity solution $g(t, \mathbf{s}, \mathbf{q})$ on $[0, T) \times \overline{\mathcal{A}}^{\prime} \times \mathbb{Z}^{n}$, as $(d t, d s, Q) \rightarrow(0,0, \infty)$, provided $d t<f(d s)$ for some $f: \mathbb{R}^{+} \rightarrow \mathbb{R}^{+}$.

Proof. Follows from Propositions 4.5.4, 4.5.5, 4.5.6, and Barles and Souganidis (1991). For completeness, the details will be provided.

The limits

$$
\begin{aligned}
& g_{*}(t, \mathbf{s}, \mathbf{q}):=\liminf _{\substack{(d t, d s, Q) \rightarrow(0,0, \infty) \\
\left(t^{\prime}, \mathbf{s}^{\prime}\right) \rightarrow(t, \mathbf{s})}} g^{d t, d s, Q}\left(t^{\prime}, \mathbf{s}^{\prime}, \mathbf{q}\right) \\
& g^{*}(t, \mathbf{s}, \mathbf{q}):=\limsup _{(d t, d s, Q) \rightarrow(0,0, \infty)} g^{d t, d s, Q}\left(t^{\prime}, \mathbf{s}^{\prime}, \mathbf{q}\right)
\end{aligned}
$$


are lower/upper semi-continuous functions on $[0, T] \times \overline{\mathcal{A}}^{\prime} \times \mathbb{Z}^{n}$, and inherit the boundedness of $\left\{g^{d t, d s, Q}\right\}$ by the stability result in Proposition 4.5.5. It suffices to show (by symmetry and the strong comparison principle) that $g_{*}$ possesses the viscosity supersolution property. To this end, let $(\bar{t}, \overline{\mathbf{s}}, \overline{\mathbf{q}}) \in[0, T) \times \overline{\mathcal{A}}^{\prime} \times \mathbb{Z}^{n}$ and $\varphi \in C_{b}^{1,2}\left([0, T] \times \overline{\mathcal{A}}^{\prime} \times \mathbb{Z}^{n}\right)$ such that $(\bar{t}, \overline{\mathbf{s}}, \overline{\mathbf{q}})$ attains the strict global minimum of $g_{*}-\varphi$. Then there exists sequences $\left\{\left(t_{k}^{\prime}, \mathbf{s}_{k}^{\prime}, \mathbf{q}_{k}^{\prime}\right)\right\}_{k}$ in $[0, T) \times \overline{\mathcal{A}}^{\prime} \times \mathbb{Z}^{n}$ and $\left\{\left(d t_{k}, d s_{k}, Q_{k}\right)\right\}_{k}$ such that

$$
\begin{aligned}
\left(t_{k}^{\prime}, \mathbf{s}_{k}^{\prime}, \mathbf{q}_{k}^{\prime}\right) & \longrightarrow(\bar{t}, \overline{\mathbf{s}}, \overline{\mathbf{q}}), \\
\left(d t_{k}, d s_{k}, Q_{k}\right) & \longrightarrow(0,0, \infty), \\
g^{d t_{k}, d s_{k}, Q_{k}} & \longrightarrow g_{*}(\bar{t}, \overline{\mathbf{s}}, \overline{\mathbf{q}}),
\end{aligned}
$$

and $\left(t_{k}^{\prime}, \mathbf{s}_{k}^{\prime}, \mathbf{q}_{k}^{\prime}\right)$ is the global minimizer of $g^{d t_{k}, d s_{k}, Q_{k}}-\varphi$.

Observe that for us to apply the monotonicity result in Proposition 4.5.4, the sequence $\left\{\left(d t_{k}, d s_{k}, Q_{k}\right)\right\}_{k}$ must have $d t_{k}$ sufficiently small for each $d s_{k}$, as prescribed by (C.9).

Denote the sequence of minimum values $\left\{\beta_{k}\right\}_{k}$ by $\beta_{k}:=\left(g^{d t_{k}, d s_{k}, Q_{k}}-\varphi\right)\left(t_{k}^{\prime}, \mathbf{s}_{k}^{\prime}, \mathbf{q}_{k}^{\prime}\right)$. Then we necessarily have the relation $g^{d t_{k}, d s_{k}, Q_{k}} \geq \varphi+\beta_{k}$. Applying the definition of the numerical scheme in (4.16) and the monotonicity result from Proposition 4.5.4 gives:

$$
\begin{aligned}
\beta_{k}+\varphi\left(t_{k}^{\prime}, \mathbf{s}_{k}^{\prime},\right. & \left.\mathbf{q}_{k}^{\prime}\right)=g^{d t_{k}, d s_{k}, Q_{k}}\left(t_{k}^{\prime}, \mathbf{s}_{k}^{\prime}, \mathbf{q}_{k}^{\prime}\right) \\
& =\mathcal{S}^{d t_{k}, d s_{k}, Q_{k}}\left(t_{k}^{\prime}+d t_{k}, \mathbf{s}_{k}^{\prime}, \mathbf{q}_{k}^{\prime}, g^{d t_{k}, d s_{k}, Q_{k}}\right) \\
& \geq \mathcal{S}^{d t_{k}, d s_{k}, Q_{k}}\left(t_{k}^{\prime}+d t_{k}, \mathbf{s}_{k}^{\prime}, \mathbf{q}_{k}^{\prime}, \varphi+\beta_{k}\right) \\
& =\mathcal{S}^{d t_{k}, d s_{k}, Q_{k}}\left(t_{k}^{\prime}+d t_{k}, \mathbf{s}_{k}^{\prime}, \mathbf{q}_{k}^{\prime}, \varphi\right)+\beta_{k} \\
& =\max \left[\mathcal{T}^{d t_{k}, d s_{k}, Q_{k}}\left(t_{k}^{\prime}+d t_{k}, \mathbf{s}_{k}^{\prime}, \mathbf{q}_{k}^{\prime}, \varphi\right), \mathcal{M}^{d t_{k}, d s_{k}, Q_{k}}\left(t_{k}^{\prime}+d t_{k}, \mathbf{s}_{k}^{\prime}, \mathbf{q}_{k}^{\prime}, \varphi\right)\right]+\beta_{k}
\end{aligned}
$$

We then have the relation

$$
\begin{aligned}
\min \left\{\frac{1}{d t_{k}}\left[\varphi\left(t_{k}^{\prime}, \mathbf{s}_{k}^{\prime}, \mathbf{q}_{k}^{\prime}\right)-\mathcal{T}^{d t_{k}, d s_{k}, Q_{k}}\left(t_{k}^{\prime}+d t_{k}, \mathbf{s}_{k}^{\prime}, \mathbf{q}_{k}^{\prime}, \varphi\right)\right]\right. \\
\left.\varphi\left(t_{k}^{\prime}, \mathbf{s}_{k}^{\prime}, \mathbf{q}_{k}^{\prime}\right)-\mathcal{M}^{d t_{k}, d s_{k}, Q_{k}}\left(t_{k}^{\prime}+d t_{k}, \mathbf{s}_{k}^{\prime}, \mathbf{q}_{k}^{\prime}, \varphi\right)\right\} \geq 0
\end{aligned}
$$

and applying the consistency result in Proposition 4.5.6 while taking $k \rightarrow \infty$ finally yields the viscosity supersolution property

$$
\begin{aligned}
& \min \left[-\frac{\partial \varphi}{\partial t}-\frac{1}{2} \sum_{i, j=1}^{n}\left[\boldsymbol{\sigma} \boldsymbol{\sigma}^{\prime}\right]_{i j} \frac{\partial^{2} \varphi}{\partial s_{i} \partial s_{j}}-\sum_{i, \pm} \frac{\lambda}{\kappa} \exp \left\{-1+\frac{\kappa \xi}{2}+\kappa \Delta \varphi\right\}+\phi \ell(\mathbf{q})\right. \\
&\left.\varphi-\max _{i, \pm}\left\{\Delta_{i}^{ \pm} \varphi-\frac{\xi_{i}}{2}\right\}\right]\left.\right|_{(\bar{t}, \overline{\mathbf{s}}, \overline{\mathbf{q}})} \geq 0 .
\end{aligned}
$$




\subsection{Conclusions}

This chapter establishes a rigorous framework for a multiple asset economy that exhibits a specific type of structural dependence, defined as being over-complete. A parsimonious model is then built upon this framework that adheres to the strict no arbitrage bounds dictated by the construction of these assets. It is shown that when these assets have nonzero bid/offer spreads, the economy no longer contains redundant assets; a fundamental difference between this framework and classical quantitative finance.

An HFT strategy is developed for the investor that both passively submits LOs and aggressively executes MOs. The corresponding stochastic optimal control problem is solved when maximizing expected terminal wealth while penalizing open positions via the investor's total portfolio risk at each point in time; the multivariate extension to penalizing via the squared position in one asset at each point in time. Qualitative features of the optimal trading strategy are investigated and inventory management is, once again, demontrated to play a key role in the success of the HFT strategy. More specifically, the investor actively submits her LO quotes in such a way to manage her inventory and utilize the instantaneous correlation between multiple assets to partially hedge away the inventory risk associated with a large open position in a less liquid asset.

Through the use of a simulation study and a 2-dimensional illustrative example, we show the positive effect that trading multiple assets together, rather than trading each one seperately, has on the investor's PnL. By using more liquid assets to partially hedge away risk, the investor can better manage her inventory risk when market making in a less liquid asset allowing her to be more aggressive, generate more trade flow and hence, increase her mean PnL for a fixed level of risk. This tradeoff between mean PnL and risk, whether it be standard deviation of PnL or mean inventory risk, is highlighted by varying the inventory aversion parameter $\phi$ and constructing the corresponding efficient frontier.

Moreover, the investor can further enhance profits (or reduce risk for a given level of mean $\mathrm{PnL}$ ) when her strategy considers where the midprice process, $\mathbf{S}_{t}$, is in the noarbitrage region. That is, when she considers the structural dependence in addition to just considering the multiple assets as correlated Brownian Motions. More specifically, she adjusts her LO quotes and may submit MOs when the midprice process approaches the boundary of the no-arbitrage region in an attempt to acquire a directional position in some particular combination of the assets.

An explicit numerical scheme is provided to solve the derived HJBQVI and convergence

results are discussed to ensure that the given discretization scheme converges to the true 
viscosity solution. Lastly, we derive a dimensional reduction in the spatial variable for the midprice which significantly reduces the computational time of the numerical solution, thus making it viable for practical application. 


\section{Chapter 5}

\section{The Generalized Latent Hawkes Process}

When analyzing event data, many natural systems exhibit large amounts of clustering. Although there are cases when such behaviour can be explained by poisson clustering ${ }^{49}$, there are several others where self-excitation effects are more pronounced and quiet periods persist far longer. The modeling of self-exciting event data has been attempted in a variety of disciplines. Notable applications include seismology (see Ogata (1988) and Vere-Jones (1995) for the modeling of earthquake data), neuroscience (see Chornoboy et al. (1988) for the modeling of neural activity), genetics and molecular biology (see Gusto and Schbath (2005) for the analysis of motif occurrences in DNA sequences via Hawkes processes), and the social sciences (see Mohler et al. (2011) for the modeling of criminal activity).

As stated earlier, the classical multivariate Hawkes process (Hawkes (1971)) has recently been used in the financial econometrics literature to model clustering in trade arrival and changes in the limit order book (LOB), see Large (2007), Bowsher (2007), Shek (2011), and Toke (2011). Embrechts et al. (2011) improve upon the classical Hawkes model by introducing dependency in the marks (in the context marked point processes) via copulas.

However, the fact is that most applications of such a process for modeling purposes do not have marks that are useful for estimating the jump size in intensity as a part of the observable data. This is the first work to provide statistical estimation methodologies for a Hawkes model with a random, unobservable, jump size in the intensity process (or, more precisely, a marked Hawkes process with unobservable marks). The fact that

\footnotetext{
${ }^{49}$ Poisson clustering refers to the fact that clusters of events are likely to occur simply by chance, even when the underlying event arrival rate is constant.
} 
these random jump sizes are latent makes classical likelihood methods computationally prohibitive.

This chapter is outlined as follows: Section 5.1 motivates this work in the context of financial microstructure. Section 5.2 presents the model, the form of the data, and some other probabilistic quantities of interest. Section 5.3 details the first calibration algorithm which focuses on renewal points (defined in Section 5.2.4) and the isolated likelihood function (defined in Section 5.3.1). Both asymptotic and sharp analytic bounds on this pseudo-likelihood function are also provided, as well as a simulation study. Section 5.4 outlines the second calibration algorithm which involves a Sequential Monte Carlo (SMC) particle filter. Section 5.5 is concerned with the construction of adapted estimators for the latent intensity process, $\boldsymbol{\lambda}_{t}$. Finally, Section 5.6 surveys other real-world applications of the Generalized Hawkes Model within the context of quantitative finance.

Many of the methods derived in this chapter will be put to use on real data in Sections 6.1 and 6.2, and are critical components in the real-world application of the high frequency trading (HFT) strategy derived in Chapter 3, as will also be applied to real data in Secton 6.3 .

\subsection{Motivation}

As previously eluded to, self-exciting point processes arise in many financial and biological applications. A point process $\left\{M_{t}\right\}_{t \geq 0}$ is typically referred to as self-exciting if $\mathbb{C}\left[M_{t+s}-\right.$ $\left.M_{t}, M_{t+s+u}-M_{t+s}\right]>0$ for all $t \geq 0$ and $s, u>0$. A particular example of such a process is the (classical) Hawkes Process where the arrival of each point induces a (constant) jump in the process's intensity rate. In many real world applications, the conjecture is that some points should induce such a (potentially random) jump, while others do not. In this chapter, we propose such a model and give an efficient calibration algorithm when the jump indicators are latent.

The main motivation for this work is for application in high frequency trading algorithms. In particular, the optimal trading strategy derived and discussed in Chapter 3 assumes that the arrival process of market orders (trades) follows this generalized Hawkes process with the jump indicator being interpreted as the trade being an influential one. Stated another way, the jump size random variable is a multiple of a binary random variable.

Observe the fitted intensity in Figure 5.1 (same as Figure 3.1, repeated here for convenience) to Level I financial data ${ }^{50}$. Notice how the generalized model drastically improves

\footnotetext{
${ }^{50}$ Level I data contains event data that corresponds to executed trades with their timestamps and
} 


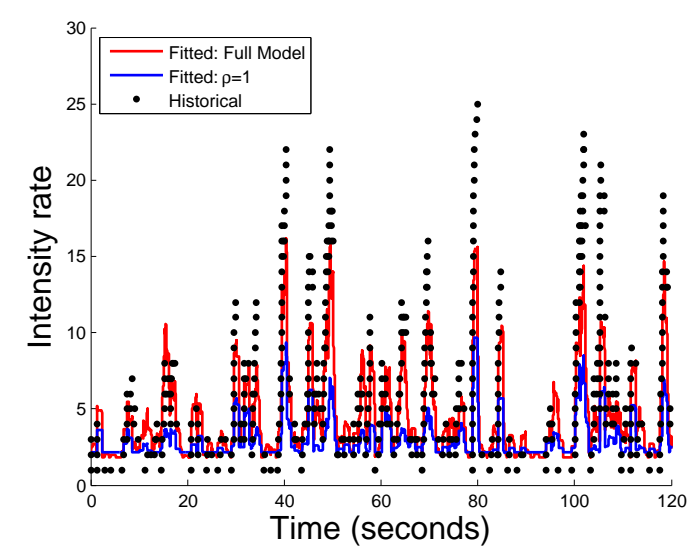

(a) Arrival of IBM market buy orders

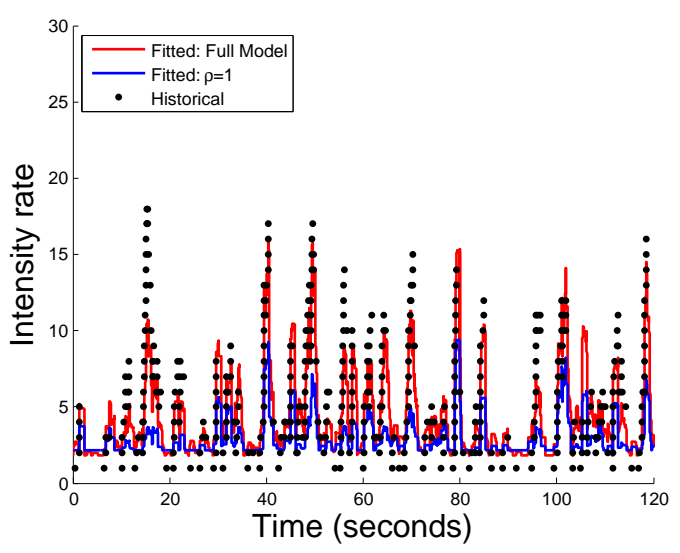

(b) Arrival of IBM market sell orders

Figure 5.1: IBM market orders. Historical running intensity versus smoothed fitted intensity using a 1 second sliding window for IBM for a 2 minute period, between 3:30pm and 3:32pm, February 1, 2008.

the fitted intensity and hence, the need to extend the model beyond the classical Hawkes model. One key feature is that the generalized model is better at capturing the sharp spikes in activity that is present in the data. The extremely low values of the historical intensities are due to the absence of points in the moving window (something that does not exist in any model with a baseline rate). The key feature that the full model is attempting to capture is the extreme clustering of events. As you can see in Figure 5.1, the full model more closely resembles the data generating process in large clusters; this will be supported with statistical evidence in Section 6.1.

The estimated parameters for the 30 minute window that contains the data in Figure 5.1 can be found in Table 5.1. The larger estimated jump sizes in the full model are compensated by the probability that not all points induce a jump in the intensity. This is what creates the sharper peaks when many points are clustered together while still preserving the overall mean activity rate. By construction of the calibration algorithm in Section 5.3.2, both methods produce the same fitted value of the long run mean activity rate $\lim _{u \rightarrow \infty} \mathbb{E}\left[\boldsymbol{\lambda}_{u} \mid \mathcal{F}_{t}\right]$, where the filtration $\mathcal{F}_{t}$ is defined in Section 5.2.2.

volumes. 


\begin{tabular}{ccc}
\hline & Classical Model & Full Model \\
\hline$\hat{\rho}$ & 1 & 0.6520 \\
$\hat{\beta}_{1}=\hat{\beta}_{2}$ & 180.05 & 195.15 \\
$\hat{\eta}_{11}=\hat{\eta}_{22}$ & 64.1589 & 111.4633 \\
$\hat{\eta}_{12}=\hat{\eta}_{21}$ & 55.7357 & 103.8997 \\
$\hat{\theta}_{1}=\hat{\theta}_{2}$ & 2.1616 & 1.8148 \\
$\hat{A}^{-1} \hat{\zeta}$ & 6.4695 & 6.4695 \\
\hline
\end{tabular}

Table 5.1: Parameter estimates used for constructing the plots in Figure 5.1.

\subsection{The Generalized Hawkes Process}

\subsubsection{The Model}

Let $\left\{\mathbf{N}_{t}\right\}_{0 \leq t \leq T}$ be a $n$-dimensional vector of counting processes with intensity process $\left\{\boldsymbol{\lambda}_{t}\right\}_{0 \leq t \leq T}$ satisfying

$$
d \boldsymbol{\lambda}_{t}=\boldsymbol{\beta}\left(\boldsymbol{\theta}-\boldsymbol{\lambda}_{t}\right) d t+\boldsymbol{\eta}_{\sum N_{i, t-}} d \mathbf{N}_{t}
$$

and initial condition $\boldsymbol{\lambda}_{0} \geq 0$. For any fixed $t$, assume $\boldsymbol{\eta}_{\sum N_{i, t-}}$ is an $n \times n$ random matrix selected from a finite distribution (independent of $t$ ), with each possible matrix having non-negative entries. From this point forward, we will use the short hand $\boldsymbol{\eta}_{t}$ when referring to $\boldsymbol{\eta}_{\sum N_{i, t-}}$ and $\boldsymbol{\eta}$ when referring to a random matrix with the same distribution (since the distribution is independent of $t$ ).

Let $p$ denote the size of the codomain of $\boldsymbol{\eta}$ and denote each element of $\boldsymbol{\eta}$ as $\eta_{i j} \geq 0$. Also, denote the probability weights as $\boldsymbol{\rho}=\left(1-\sum_{i=1}^{p-1} \rho_{i}, \rho_{1}, \ldots, \rho_{p-1}\right)^{\prime}$. We also have the constraints $\boldsymbol{\theta} \in \mathbb{R}^{n}$ has positive entries, $\boldsymbol{\beta}$ is an invertible $n \times n$ matrix with positive entries, $\boldsymbol{\rho} \in(0,1]^{p}$ with $\boldsymbol{\rho} \cdot \mathbf{1}_{n}=1$, and $\boldsymbol{\beta}-\mathbb{E}[\boldsymbol{\eta}]$ has strictly positive eigenvalues. When $p=2$, we will use $\rho$ when referring to $\rho_{1}$ as there is only one degree of freedom in the $\boldsymbol{\rho}$ vector.

The terms in the SDE (5.1) can be interpreted as follows: $\boldsymbol{\theta}$ is the baseline intensity that $\boldsymbol{\lambda}_{t}$ mean-reverts to, and the speed of this mean reversion is dictated by the decay matrix $\boldsymbol{\beta}$. Hence, the term $\boldsymbol{\beta}\left(\boldsymbol{\theta}-\boldsymbol{\lambda}_{t}\right) d t$ is responsible for exponential decay to level $\boldsymbol{\theta}$. The factor $\boldsymbol{\eta}_{t}$ is the random jump matrix associated with the event at time $t$ that causes jump in the intensity process $\boldsymbol{\lambda}_{t}$ and may induce subsequent event arrivals (thus creating a self-excitation effect).

For each entry $\eta_{i j}$ of the random matrix, we denote its possible values as $\eta_{i j}(k)$ for $k=0,1, \ldots, p-1$ (and the full matrix denoted similarly as $\boldsymbol{\eta}(k)$ ) which has associated 
probability $\rho_{k}$ or, in the case of $k=0$, has probability $1-\sum_{i=1}^{p-1} \rho_{i}$. It typically makes sense to force $\eta_{i j}(k)$ to be monotonic non-decreasing in $k$, but we do not require this as there may be some practical applications where having the reversed order for some $i, j$ makes sense. Finally, observe that since the process $\mathbf{N}_{t} \in \mathbb{Z}^{n}$, we only require a countable number of each of the $\eta_{i j}$, random variables and therefore have no technical issues in constructing such a well defined probability space.

Observe that if $\boldsymbol{\rho}=1$ (and $\eta_{i j}(0)=0$ for all $i, j$ ), then the model reduces to a poisson process. In particular, if $\boldsymbol{\lambda}_{0}=\boldsymbol{\theta}$, then the corresponding poisson process is homogeneous. If $\boldsymbol{\rho}=1$ with non-zero $\boldsymbol{\eta}(0)$, then the model reduces to the Classical Hawkes process where every event induces a constant jump in $\boldsymbol{\lambda}_{t}$. Note the self-excitation effect of a jump of size $\eta_{i i}$, as well as the cross-excitation effect of a jump of size $\eta_{i j}(i \neq j)$ in the other intensity processes. This simpler model is referred to as the Classical Hawkes Model (or the $\rho=1$ model).

In real world applications, the random matrix $\boldsymbol{\eta}$ is typically unobservable. However, since it has a direct effect on the distribution of the point process $\mathbf{N}_{t}$, we can use statistical inference to aid in parameter estimation and the likelihood of potential paths of $\boldsymbol{\lambda}_{t}$.

\subsubsection{The Data}

We will assume that data (which we aim to calibrate the generalized Hawkes model to) is provided in the following form: Let $T_{1}, \ldots, T_{N}$ denote the inter-arrival times of the data points. More precisely, each $T_{i}$ is an element of $[0, T] \times\{1, \ldots, n\}$, with the first component being the inter-arrival time of the $i^{\text {th }}$ event and the second representing to which element of the process $\mathbf{N}_{t}$ the arrival point corresponds. Let $t_{i} \in[0, T]$ be the corresponding $i^{\text {th }}$ event time, with the second component being the same as that of $T_{i}$.

Define the (completed, right-continuous) filtrations:

$$
\begin{aligned}
\overline{\mathcal{F}}_{t} & =\sigma\left(\left\{\mathbf{N}_{u}\right\}_{0 \leq u \leq t}\right), \\
\mathcal{F}_{t} & =\overline{\mathcal{F}}_{t} \vee \sigma\left(\left\{\boldsymbol{\eta}_{u}\right\}_{0 \leq u \leq t}\right) .
\end{aligned}
$$

$\overline{\mathcal{F}}_{t}$ is the natural filtration generated by $\mathbf{N}_{t}$ (i.e. it only contains information about the arrival of points in $\mathbf{N}_{t}$ ), while $\mathcal{F}_{t}$ also contains information about the jump sizes $\boldsymbol{\eta}_{t}$ in the intensity process $\boldsymbol{\lambda}_{t}$. Conditional on $\overline{\mathcal{F}}_{t}$, it is not possible to determine the intensity process on the interval $[0, t]$; however, expanding the filtration to $\mathcal{F}_{t}$ allows one to extract the exact intensity path. 
Note that the random jump size $\boldsymbol{\eta}_{t_{i}}$ is not included in the observable data (that is, we observe the filtration $\overline{\mathcal{F}}_{t}$, not $\mathcal{F}_{t}$ ). This detail is what sets this work apart from previously published estimation techniques. Although we do not observe $\boldsymbol{\eta}_{t_{i}}$, we can still make inference about it by observing the effect it has on future event arrivals of the process $\mathbf{N}_{t}$ for $t>t_{i}$.

\subsubsection{Probabilistic Quantities of Interest}

Observation 5.2.1. The solution to (5.1) is given by

$$
\boldsymbol{\lambda}_{t}=\boldsymbol{\theta}+e^{-\boldsymbol{\beta} t}\left(\boldsymbol{\lambda}_{0}-\boldsymbol{\theta}\right)+\int_{0}^{t} e^{-\boldsymbol{\beta}(t-u)} \boldsymbol{\eta}_{u} d \mathbf{N}_{u}
$$

where $\boldsymbol{\eta}_{t}$ is shorthand for $\boldsymbol{\eta}_{\sum N_{i, t-}}$.

Applying Itô's Lemma for jump processes to (5.4) gives Equation (5.1) directly. Also notice that since $\mathbf{N}_{t}$ is a simple function on compact sets ( $\mathbb{P}$ - a.s.), the above integral collapses to a finite sum containing $\mathbf{N}_{t} \cdot \mathbf{1}_{n}$ terms (where $\mathbf{1}_{n}$ denotes the $n$-dimensional vector of 1's), of which only those that have positive jump size are non-zero.

Observation 5.2.2. The long run mean (LRM) activity rate can be computed and is independent of the current level of intensities:

$$
\lim _{u \rightarrow \infty} \mathbb{E}\left[\boldsymbol{\lambda}_{u} \mid \mathcal{F}_{t}\right]=\left(\mathbb{I}_{n}-\boldsymbol{\beta}^{-1} \mathbb{E}[\boldsymbol{\eta}]\right)^{-1} \boldsymbol{\theta}
$$

There are also probabilistic quantities that are not only interesting in their own right, but also will be of use in particle filtering methods to be discussed in Section 5.5.2.

Observation 5.2.3. The function $g(t, l \mid y):=\mathbb{P}\left[T_{k}(1) \in d t, T_{k}(2)=l \mid \boldsymbol{\lambda}_{t_{k-1}}=y\right]$ admits the representation

$$
g(t, l \mid y)=\exp \left\{-\left(\boldsymbol{\theta} t+\boldsymbol{\beta}^{-1}\left(\mathbb{I}_{n}-e^{-\boldsymbol{\beta} t}\right)(y-\boldsymbol{\theta})\right) \cdot \mathbf{1}_{n}\right\} \cdot\left[e^{-\boldsymbol{\beta} t}(y-\theta) \cdot e_{l}+\theta_{l}\right] d t
$$

for $t \geq 0$ and $l \in\{1, \ldots, n\}$, where $e_{i}$ is the $n$-dimensional unit vector with 1 in the $i^{\text {th }}$ component. The survival analogue $g_{\text {surv. }}(t \mid y):=\mathbb{P}\left[T_{k}(1)>t \mid \boldsymbol{\lambda}_{t_{k-1}}=y\right]$ is given by

$$
g_{\text {surv. }}(t \mid y)=\exp \left\{-\left(\boldsymbol{\theta} t+\boldsymbol{\beta}^{-1}\left(\mathbb{I}_{n}-e^{-\boldsymbol{\beta} t}\right)(y-\boldsymbol{\theta})\right) \cdot \mathbf{1}_{n}\right\} .
$$

The function $g(t, l \mid y)$ in Equation (5.6) represents the probability density of observing 
the event $T_{k}=(t, l)$, given the value of $\boldsymbol{\lambda}_{t}$ at the $(k-1)^{\text {th }}$ event. The function $g_{\text {surv. }}(t \mid y)$ in Equation (5.7) is the survivor analogue of this quantity representing the probability that the $k^{\text {th }}$ interarrival time is greater than $t$.

Observation 5.2.4. The function $l\left(q \mid y, S_{1}, S_{2}\right):=\mathbb{P}\left[\boldsymbol{\eta}_{t_{k}}=\boldsymbol{\eta}_{S_{1}(1)}(q) \mid \boldsymbol{\lambda}_{t_{k-1}}=y, T_{k}=\right.$ $\left.S_{1}, T_{k+1}=S_{2}\right]$, for $q \in\{0,1, \ldots, p-1\}$ and $y \in \mathbb{R}^{n}$, admits the representation

$$
l\left(q \mid y, S_{1}, S_{2}\right)=\frac{\rho_{q} \cdot g\left(S_{2} \mid z_{q}(y)\right)}{\sum_{r=0}^{p-1} \rho_{r} \cdot g\left(S_{2} \mid z_{r}(y)\right)}
$$

with $z_{r}(y)=\boldsymbol{\theta}+e^{-\boldsymbol{\beta} s_{1}(1)}(y-\boldsymbol{\theta})+\boldsymbol{\eta}_{S_{1}(1)}(r) e_{S_{1}(2)}$. The survival analogue $l\left(q \mid y, S_{1}, S_{2}\right):=$ $\mathbb{P}\left[\boldsymbol{\eta}_{t_{k}}=\boldsymbol{\eta}_{S_{1}(1)}(q) \mid \boldsymbol{\lambda}_{t_{k-1}}=y, T_{k}=S_{1}, T_{k+1}(1)>S_{2}(1)\right]$ is obtained by simply replacing $g(\cdot)$ with $g_{\text {surv. }}(\cdot)$ in Equation (5.8).

The function $l\left(q \mid y, S_{1}, S_{2}\right)$ in Equation (5.8) represents the posterior probability that the jump induced by the $k^{\text {th }}$ event is of type $q$, given the intensity at $t_{k-1}$, and the interarrival times $T_{k}$ and $T_{k+1}$.

\subsubsection{Renewal Points}

Definition 5.2.5. A data point $t_{k}$ is said to be an $\epsilon$-renewal point if $\sum_{i=1}^{n} \frac{\lambda_{i, t_{k}-}-\theta_{i}}{\theta_{i}}<\epsilon$, where $\epsilon>0$ is a free parameter.

Observe that if $t_{k}$ is an $\epsilon$-renewal point, then $\boldsymbol{\lambda}_{t}$, for $t \geq t_{k}$, is approximately (for all numerical intents and purposes) independent of $\mathcal{F}_{t_{k-1}}$ (and hence, $\overline{\mathcal{F}}_{t_{k-1}}$ as well by containment). This approximation is used in Section 5.3 to efficiently compute likelihoods. We will also drop the dependence on $\epsilon$ when referring to renewal points as we will fix $\epsilon>0$ at the beginning of the calibration.

Definition 5.2.6. A cluster of events, denoted $C_{k}^{m}$, is the set of consecutive points $\left\{t_{k}, t_{k+1}, \ldots, t_{k+m}\right\}$ with the property that $t_{k}$ is a renewal point. Although (with probability one) there is no point at $t=0$, we define $t_{0}=0$ (so that the set $C_{0}^{m}$ makes sense). 


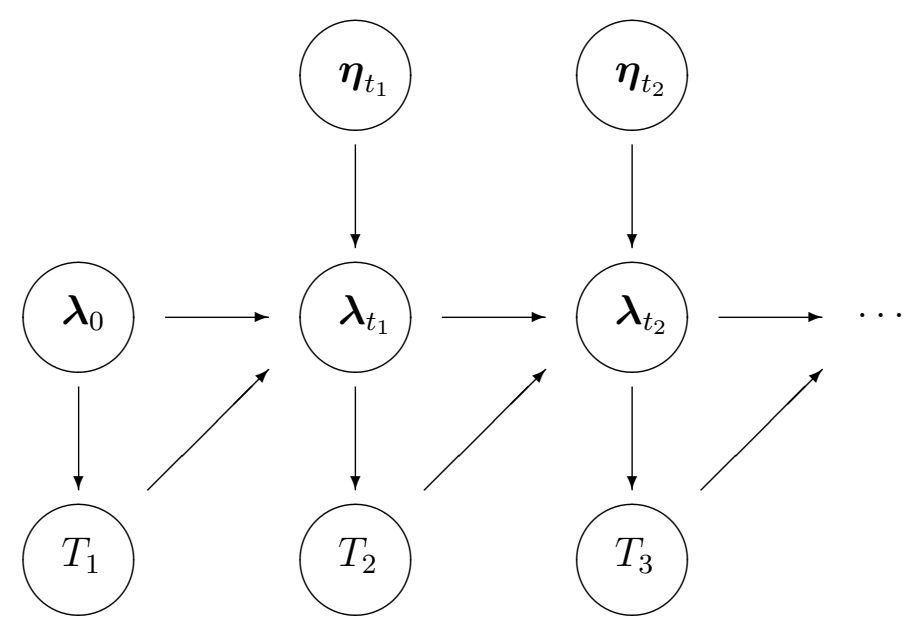

Figure 5.2: A graphical model representation of dependencies in our self-exciting process with random jump sizes $\boldsymbol{\eta}_{t_{k}}$.

\subsection{Calibration Algorithm I: Isolated Likelihood}

Finding traditional maximum likelihood estimators (MLEs) is not numerically feasible due to the large number of possible sequences $\left\{\boldsymbol{\eta}_{t_{k}}\right\}_{k \in \mathbb{N}}$. For a graphical model representation of dependencies, see Figure 5.2. The exact likelihood function has computation time $O\left(p^{N}\right)$. For numerical computations, the run time can be reduced to $\frac{1}{m(N)} p^{m(N)}$ (up to a multiplicative constant), where $m(N)$ is the size of the largest cluster of points, where $\epsilon$ is taken to reflect floating point precision, with only the first point being a renewal point. Although this is still too slow for applications in high freqeuency finance, where $N$ can easily be $10^{4}$ and $m\left(10^{4}\right)$ can still be far too large for practical implementations, this discussion serves as a motivation for the algorithm developed in Section 5.3.2.

The goal of this calibration algorithm is as follows: We want to find the maximizer $\hat{x}$ of a function $f(x)$. Unfortunately, $f$ has exponential computational time (in the total number of data points, $N)$. We wish to find a function $g(x)$ that has $P$-time computation and the same maximizer as $f$. Such a problem, in general, is extremly difficult to solve. However, we exploit the structure of the function $f$ to construct $g$ quickly and efficiently without the need to compute $f$ even once. In particular, we exploit the probabilistic structure of the model that generates the likelihood function $f$.

In what follows, we propose an efficient method for calibration to medium to large datasets via maximizing a pseudo-likelihood function and provide an analytic bound on how close this function is to the true likelihood function. 


\subsubsection{The Isolated Likelihood Function $\tilde{\mathcal{L}}$}

By construction of the sequence in Step 4 of Section 5.3.2, the observations in the sequence (each element being a consecutive sequence of $m$ points) are almost mutually independent. The smaller $\epsilon$ is, the better this approximation will be, but the number of renewal points will decrease.

Assuming the observations in the sequence are mutually independent and hence, $\underline{\boldsymbol{\lambda}_{t}}$ is $\underline{\text { restarted at level } \boldsymbol{\theta}}$, the isolated log-likelihood (LLH) for each individual element (referred to as a cluster) is explicitly:

$$
\log \tilde{\mathcal{L}}=\log \left\{\sum_{H} \tilde{\mathcal{L}}(H)\right\}=\log \left\{\sum_{H} \prod_{k=1}^{m} \rho_{H_{k}} \cdot \lambda_{T_{a_{k}}(2), t_{a_{k}}}(H) \cdot \mathbb{P}\left[\tau>T_{a_{k}}(1) \mid H\right]\right\}
$$

where $\left\{a_{k}\right\}_{k=0,1, \ldots, m}$ is the sequence of points within a given cluster, the random variable $\tau$ is the time until the next event, $H \in \mathbb{Z}^{m}$ has $j^{\text {th }}$ component corresponding to which jump size trade $j$ had (i.e. $H_{j} \in\{0,1, \ldots, p-1\}$ ), $\lambda_{i, t}$ is a function of the sequence $H$ via the random matrices $\boldsymbol{\eta}^{(H)}$ and simplifies Equation (5.4) to

$$
\lambda_{i, t}(H)=\left[\boldsymbol{\theta}+\int_{t_{a_{0}}}^{t} e^{-\boldsymbol{\beta}(t-u)} \boldsymbol{\eta}_{u}^{(H)} d \mathbf{N}_{u}\right] \cdot e_{i}
$$

and $\mathbb{P}\left[\tau>T_{a_{k}}(1) \mid H\right]$ is also approximated by

$$
\mathbb{P}\left[\tau>T_{a_{k}}(1) \mid H\right]=\exp \left\{-\left(\boldsymbol{\theta} T_{a_{k}}(1)+\boldsymbol{\beta}^{-1}\left(\mathbb{I}_{n}-e^{-\boldsymbol{\beta} T_{a_{k}}(1)}\right)\left(\boldsymbol{\lambda}_{t_{a_{k-1}}}(H)-\boldsymbol{\theta}\right)\right) \cdot \mathbf{1}_{n}\right\}
$$

Recall that $T_{k}(1)$ refers to the $k^{\text {th }}$ inter-arrival time and $T_{k}(2)$ refers to which process this event belongs to. The probabilities in (5.9) are easily computable analytically and the number of terms in the sum is $p^{m}$.

The probabilistic argument for spliting the data into such clusters is similar to a renewal one: When both intensity processes get close to $\boldsymbol{\theta}$ (i.e. the sum of their relative errors is less than $\epsilon$ ), the next point is assumed to have come from a "restarted" process where the intensity is equal to $\boldsymbol{\theta}$. For small $\epsilon$, since decay is exponential, this approximation proves to be both useful and accurate.

Notice that this method always results in the under-estimation of $\boldsymbol{\lambda}$. Altering the algorithm to assign any other value to $\boldsymbol{\lambda}$ such that $\theta_{i} \leq \lambda_{i} \leq \theta_{i}(1+\epsilon)$ appears to offer no discernable improvement in results for any reasonably small choice of $\epsilon$. 


\subsubsection{The Calibration Algorithm}

The calibration of the Generalized Hawkes process to data can be perfomed efficiently via the following fixed-point seeking algorithm. To ensure we have a unique MLE, we impose the condition that $\eta_{i j}(k)$, for fixed $i, j$, is a strictly monotonic function in $k$. To increase numerical stability in some datasets, as well as maintain identifiability ${ }^{51}$, one may want additional constraints of the form $\left|\eta_{i j}(k)-\eta_{i j}(k-1)\right|>\delta_{i j}>0$ and $\rho_{i}>\delta_{i}>0$.

The calibration methodology can be described as seeking the fixed point of

$$
Q\left(\hat{\xi} \mid T_{1}, \cdots, T_{N}\right)=\hat{\xi} \quad \text { with } \quad Q(\xi \mid Y)=\underset{\zeta}{\operatorname{argsup}} \overline{\mathcal{L}}(\zeta ; \xi, Y)
$$

where $\overline{\mathcal{L}}$ is a pseudo-likelihood function that is the product of isolated likelihood functions of the form of (5.9). The following algorithm provides an efficient way to do this.

1. Fit the data $\left\{T_{j}\right\}_{j}$ via maximum likelihood restricting the maximizer to be on the $\boldsymbol{\rho}=\{1,0,0, \ldots, 0\}$ slice of the parameter space. This can be done easily as the likelihood can be efficiently computed since the intensity path is uniquely determined given the data $\left\{T_{j}\right\}_{j}$. Appendix E.1 outlines this procedure for the model described in Section 3.2.2 and is easily generalized.

2. Select free parameters $\epsilon=\frac{1}{100}$, and initial guess for $\boldsymbol{\rho}$ which we take to be $\boldsymbol{\rho}_{0}=\frac{1}{p} \mathbf{1}_{p}$. With some abuse of notation, compute initial estimate of the codomain of $\boldsymbol{\eta}$, namely $\boldsymbol{\eta}_{0}$ which is composed of $\boldsymbol{\eta}_{0}(0), \boldsymbol{\eta}_{0}(1), \ldots, \boldsymbol{\eta}_{0}(p-1)$, by taking $\boldsymbol{\eta}_{0}(j)=\frac{2 j}{p(p-1)} \boldsymbol{\nu}$ where $\boldsymbol{\nu}$ is the $\boldsymbol{\rho}=1$ estimate of $\boldsymbol{\eta}$ from step 1. Take $\boldsymbol{\beta}_{0}$ and $\boldsymbol{\theta}_{0}$ as in step 1. Finally, set $k=0$.

3. Compute the upper bound of the intensity path, i.e. the path when $\eta_{i j, t_{q}}=$ $\max _{l}\left\{\eta_{i j}(l)\right\}$ for all $q$ using the current parameter estimates. Observe that this intensity function dominates all potential intensity functions based on some jumps being potentially less than the maximum used in the computation (for the fixed parameter set used).

4. Select the likelihood cluster size $m \in \mathbb{N}$ and Construct a set of clusters of size $m$, $\left\{C_{k}^{j}\right\}_{j \in \mathbb{I}}$, where $\mathbb{I} \subset\{0,1,2, \ldots, n\}$ is defined by the following recursive criterion: $0 \in \mathbb{I}, 1,2,3, \ldots,(m-1) \notin \mathbb{I}$, and $j \in \mathbb{I}$ (for $j>m-1$ ) whenever $t_{j}$ is a renewal point and $(j-m+1), \ldots,(j-1) \notin \mathbb{I}$.

5. Due to the condition in step 4, likelihood maximization can now be performed in a rather efficient way. We want to maximize the isolated likelihood function over

\footnotetext{
${ }^{51} \mathrm{~A}$ family of models is identifiable if for any arbitrary $h, \mathbb{E}^{\Theta_{1}}[h(\cdot)]=\mathbb{E}^{\Theta_{2}}[h(\cdot)]$ implies $\Theta_{1}=\Theta_{2}$. (i.e. no two distinct models produce the same finite dimensional distributions).
} 
a specific $\left(n^{2}-1+\left(n^{2}+1\right) p\right)$-dimensional subset of the parameter space. If $\boldsymbol{\rho}$, $\boldsymbol{\beta}$, and (the possible values of) $\boldsymbol{\eta}$ are free parameters in the isolated likelihood function (given by Equation (5.9) in Section 5.3.1), then, by using Observation 5.2 .2 , we constrain the long run mean to remain unchanged by setting

$$
\boldsymbol{\theta}=\left(\mathbb{I}_{n}-\boldsymbol{\beta}^{-1} \mathbb{E}[\boldsymbol{\eta} \mid \Theta]\right)\left(\mathbb{I}_{n}-\boldsymbol{\beta}_{k}^{-1} \mathbb{E}\left[\boldsymbol{\eta} \mid \Theta_{k}\right]\right)^{-1} \boldsymbol{\theta}_{k}
$$

where we use $\Theta$ to denote an element of the parameter space.

After optimization, set $k=k+1$, set $\boldsymbol{\rho}_{k}, \boldsymbol{\beta}_{k}$, and $\boldsymbol{\eta}_{k}$ to the maximizers of the isolated likelihood function, and

$$
\boldsymbol{\theta}_{k}=\left(\mathbb{I}_{n}-\boldsymbol{\beta}_{k}^{-1} \mathbb{E}\left[\boldsymbol{\eta} \mid \Theta_{k}\right]\right)\left(\mathbb{I}_{n}-\boldsymbol{\beta}_{k-1}^{-1} \mathbb{E}\left[\boldsymbol{\eta} \mid \Theta_{k-1}\right]\right)^{-1} \boldsymbol{\theta}_{k-1}
$$

6. If $\left\|\boldsymbol{\rho}_{k}-\boldsymbol{\rho}_{k-j}\right\|_{2}$ is less than some tolerance (denoted $\rho$-tolerance) for $j=1,2,3$, and if $\left\|\boldsymbol{\beta}_{k}-\boldsymbol{\beta}_{k-j}\right\|$ is less than some other tolerance (denoted $\beta$-tolerance) for $j=1,2,3$, then break the sequence. We take these tolerances to be 0.001 and 0.1 , respectively.

If $k$ reaches an upper bound (we use 9), then break the sequence.

Otherwise, go to step 3.

The constraints in Step 5 are to improve the stability and speed of the algorithm as the long run mean is fixed in Step 1. As it turns out, and will be confirmed numerically, the $\boldsymbol{\rho}=1$ initial calibration does very well in identifying the correct long run mean of the model, and we leave the rest of the algorithm to identify where on this subset of the parameter space the true model resides.

The checks for convergence to a fixed point in Step 6 can be modified to the reader's content, as well as the choice of which matrix norm to use. In the simulation study in Section 5.3.5, we actually consider each component of $\boldsymbol{\beta}$ seperately for convenience. We will report the distribution of the break time for simulated data in Section 5.3.5, which confirms that 9 iterations is sufficient. One thing to keep in mind is that the function in (5.12) is not continuous in $\xi$, so a fixed point within the specified tolerances may not always exist. However, as will be numerically demonstrated later on in this chapter, such cases result in oscillation between a few values that are quite close together. This case corresponds to when the true MLE is close to a point that causes as event to switch between being a renewal point and not being one. 


\subsubsection{Bounding the Error of the Isolated Likelihood Function}

The renewal point argument given in Section 5.3.1 deserves some technical justification. We wish to investigate the difference between the approximation of the likelihood of such a cluster, $\tilde{\mathcal{L}}$, and its true likelihood, $\mathcal{L}$.

Theorem 5.3.1. Asymptotic Bound. The absolute error of the mis-specified (isolated) likelihood function is

$$
|\tilde{\mathcal{L}}-\mathcal{L}|=O(\epsilon)
$$

for small $\epsilon>0$.

Proof. See Appendix C.9.

Theorem 5.3.2. Absolute Error Bound. If $\boldsymbol{\beta}$ is a diagonal matrix, then we also have explicit absolute bounds

$$
-m \log (1+\epsilon)<\log \tilde{\mathcal{L}}-\log \mathcal{L}<m \epsilon\|\boldsymbol{\theta}\| \sum_{j=1}^{n} \beta_{j j}^{-1} .
$$

Proof. See Appendix C.10.

For general distribution of $\boldsymbol{\eta}$, the bounds in Equation (5.14) are sharp. However, it is worthy to note that for a pre-specified distribution of $\boldsymbol{\eta}$, these bounds can be improved upon.

\subsubsection{Link to Composite Likelihood Methods}

Motivated by composite likelihood functions ${ }^{52}$ associated with Gaussian random fields, which uses the big blocks method (see Caragea and Smith (2007)), the data is split into approximately independent blocks.

The Composite Marginal Likelihood (see Chandler and Bate (2007)) formed by the product of densities for all the observations in each block and is given by

$$
\mathcal{L}_{M C}(\Theta ; y)=\prod_{b=1}^{B} f\left(z_{b} ; \Theta\right)
$$

\footnotetext{
${ }^{52}$ For a general survey of composite likelihood functions, see Varin et al. (2011).
} 
where $z_{1}, \ldots, z_{B}$ are $B$ blocks of data and $f(z ; \Theta)$ is the marginal density of the block $z$.

In our framework, we choose the blocks of the form $\left\{t_{l}, \ldots, t_{l+s}\right\}$ via the following criteria: (i) $t_{l}$ and $t_{l+s}$ are renewal points and (ii) $t_{l+1}, \ldots, t_{l+s-1}$ are not renewal points.

In the calibration algorithm described in Section 5.3.2, we have approximated $f\left(z_{b} ; \Theta\right)$ by $f\left(z_{b}^{\prime} ; \Theta\right.$ ), where $z_{b}^{\prime}$ contains only the first $m$ interarrival times of $z_{b}$ (or all of $z_{b}$ if $m \geq s-1$ ). In the setup of this calibration algorithm, this density can be computed exactly with reasonable computational time and provides a stable large sample calibration.

\subsubsection{Numerical Results on Simulated Data}

We find that this calibration algorithm works remarkably well (and efficiently) when $p=2$ and $\boldsymbol{\eta}(0)=\mathbf{0}$. It is worthy to note that the generalized Hawkes model proposed here is already more general than the model posed in Section 3.2.2 since it does not assume symmetry of the parameters between the two components of $\mathbf{N}_{t}$, not to mention that the algorithm is for general dimension $n$. This section only reports results for $n=2$ as that was our motivating example. We restrict $\boldsymbol{\beta}$ to be diagonal in the calibration to maintain the bounds in Equation (5.13), and further take $\boldsymbol{\beta}$ to be a multiple of the identity matrix for simulation speed (note that this restriction is not imposed on $\boldsymbol{\beta}$ in the calibration).

We first present calibration results for the symmetric model posed in Section 3.2.2 of the single asset market making problem. See Table 5.2 for means and standard deviations of our estimators using the calibration algorithm outlined in Section 5.3.2. Estimates for the mean jump size and long run mean are also reported. Notice that as we increase the sample size $N$ from 500 to 2,000 to 10,000, we get a rapid reduction in the variance of each estimator. The histograms in Figure 5.3 show the empirical distribution of the parameter estimates for simulated data for $m=3$ on 2,000 data points.

Also notice that as we increase $m$ from 1 to 4 , we attain both a reduction in bias and in the variance of each estimator in larger datasets $(N>2,000)$ - although this is at the expense of runtime. An additional benefit of using a larger cluster size $m$ comes by way of a more stable fixed-point search, as will be discussed later in this section.

See Table 5.3 for means and standard deviations of our estimators when we do not assume symmetry (which yields 9 free parameters). Observe that the standard errors of the estimators has increased slightly over the symmetric case as a result of the increase in the number of parameters being estimated. The histograms in Figure 5.4 show the empirical distribution of estimators on simulated data for $m=3$ on 2,000 data points. 


\begin{tabular}{|c|c|c|c|c|c|c|c|c|c|}
\hline \multicolumn{10}{|c|}{$N=500$} \\
\hline & \multirow[t]{2}{*}{ true } & \multicolumn{2}{|c|}{$m=1$} & \multicolumn{2}{|c|}{$m=2$} & \multicolumn{2}{|c|}{$m=3$} & \multicolumn{2}{|c|}{$m=4$} \\
\hline & & mean & (std) & mean & (std) & mean & $(\mathrm{std})$ & mean & (std) \\
\hline$\widehat{\rho}$ & 0.7 & 0.753 & $(0.190)$ & 0.736 & $(0.177)$ & 0.729 & $(0.177)$ & 0.729 & $(0.168)$ \\
\hline$\widehat{\beta}$ & 50 & 52.13 & (10.99) & 50.86 & $(9.05)$ & 50.30 & $(8.15)$ & 50.63 & $(7.53)$ \\
\hline$\widehat{\eta}_{11}(1)$ & 25 & 25.59 & $(9.07)$ & 25.36 & $(7.93)$ & 25.46 & $(7.37)$ & 25.15 & ( 6.63$)$ \\
\hline$\widehat{\eta}_{12}(1)$ & 15 & 15.26 & $(5.91)$ & 15.18 & $(5.18)$ & 15.05 & $(4.61)$ & 15.23 & $(4.22)$ \\
\hline$\widehat{\theta}$ & 1 & 1.008 & $(0.153)$ & 1.003 & $(0.146)$ & 1.016 & $(0.137)$ & 1.012 & $(0.141)$ \\
\hline $\mathbb{E}\left[\eta_{11} \mid \widehat{\Theta}\right]$ & 17.5 & 18.06 & $(4.27)$ & 17.67 & $(3.64)$ & 17.60 & $(3.26)$ & 17.53 & $\overline{(3.18)}$ \\
\hline$L R M$ & 2.273 & 2.291 & $(0.278)$ & 2.287 & $(0.295)$ & 2.319 & $(0.289)$ & 2.305 & $(0.283)$ \\
\hline runtime & & 1.55 & $(0.79)$ & 1.44 & $(0.79)$ & 1.52 & $(0.87)$ & 1.86 & $(1.15)$ \\
\hline \multicolumn{10}{|c|}{$N=2,000$} \\
\hline$\widehat{\rho}$ & 0.7 & 0.720 & $(0.113)$ & 0.714 & $(0.104)$ & 0.709 & $(0.093)$ & 0.716 & $(0.097)$ \\
\hline$\widehat{\beta}$ & 50 & 50.28 & $(5.65)$ & 50.28 & $(4.52)$ & 50.28 & $(4.05)$ & 50.27 & ( 3.69$)$ \\
\hline$\widehat{\eta}_{11}(1)$ & 25 & 24.97 & $(4.83)$ & 25.14 & $(4.02)$ & 25.04 & $(3.61)$ & 24.91 & $(3.54)$ \\
\hline$\widehat{\eta}_{12}(1)$ & 15 & 14.97 & ( 3.07$)$ & 14.97 & $(2.49)$ & 15.09 & $(2.36)$ & 14.96 & $(2.26)$ \\
\hline$\widehat{\theta}$ & 1 & 0.999 & $(0.078)$ & 1.001 & $(0.073)$ & 1.006 & $(0.069)$ & 1.002 & $(0.068)$ \\
\hline $\mathbb{E}\left[\eta_{11} \mid \widehat{\Theta}\right]$ & 17.5 & 17.58 & $(2.16)$ & 17.64 & $(1.80)$ & 17.50 & $(1.65)$ & 17.56 & $(1.54)$ \\
\hline$L R M$ & 2.273 & 2.276 & $(0.138)$ & 2.281 & $(0.139)$ & 2.281 & $(0.136)$ & 2.276 & (0.137) \\
\hline runtime & & 2.51 & $(0.91)$ & 2.50 & $(1.03)$ & 3.57 & $(1.47)$ & 5.41 & $(2.17)$ \\
\hline \multicolumn{10}{|c|}{$\bar{c} N=10,000$} \\
\hline$\widehat{\rho}$ & 0.7 & 0.705 & $(0.049)$ & 0.704 & $(0.044)$ & 0.703 & $(0.044)$ & 0.703 & $(0.043)$ \\
\hline$\widehat{\beta}$ & 50 & 50.21 & $(2.37)$ & 50.10 & $(2.00)$ & 50.12 & $(1.82)$ & 50.00 & ( 1.63$)$ \\
\hline$\widehat{\eta}_{11}(1)$ & 25 & 25.03 & $(2.15)$ & 25.01 & $(1.71)$ & 25.02 & ( 1.63$)$ & 24.98 & $(1.62)$ \\
\hline$\widehat{\eta}_{12}(1)$ & 15 & 15.03 & $(1.34)$ & 14.93 & $(1.09)$ & 15.02 & ( 0.98$)$ & 14.96 & $(0.98)$ \\
\hline$\widehat{\theta}$ & 1 & 1.000 & $(0.034)$ & 1.001 & $(0.032)$ & 1.002 & $(0.030)$ & 1.000 & $(0.031)$ \\
\hline $\mathbb{E}\left[\eta_{11} \mid \widehat{\Theta}\right]$ & 17.5 & 17.55 & $(0.92)$ & 17.55 & $(0.77)$ & 17.52 & $(0.71)$ & 17.49 & $(0.66)$ \\
\hline$L R M$ & 2.273 & 2.272 & $(0.063)$ & 2.272 & $(0.063)$ & 2.276 & $(0.063)$ & 2.272 & $(0.064)$ \\
\hline runtime & & 5.18 & $(1.64)$ & 5.76 & $(1.77)$ & 10.03 & ( 3.68$)$ & 18.98 & $(7.86)$ \\
\hline
\end{tabular}

Table 5.2: Mean and standard deviations of estimators (1,000 simulations) when $n=$ $p=2, \boldsymbol{\eta}(0)=\mathbf{0}, \eta_{11}(1)=\eta_{22}(1), \eta_{12}(1)=\eta_{21}(1), \beta_{1}=\beta_{2}, \theta_{1}=\theta_{2}$, and using Algorithm I. Total number of free parameters is 5. LRM refers to the long run mean of activity rate of any component of $\mathbf{N}_{t}$ and runtime is in seconds. 




(a) Frequency of $\widehat{\rho}$

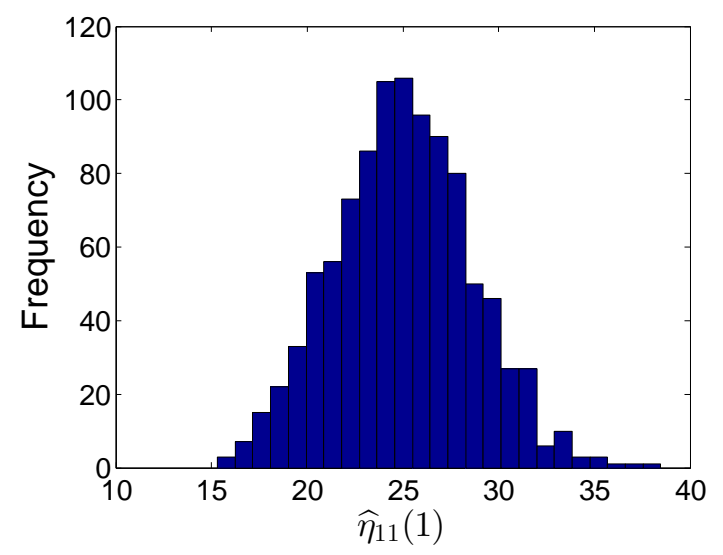

(c) Frequency of $\widehat{\eta}_{11}(1)$

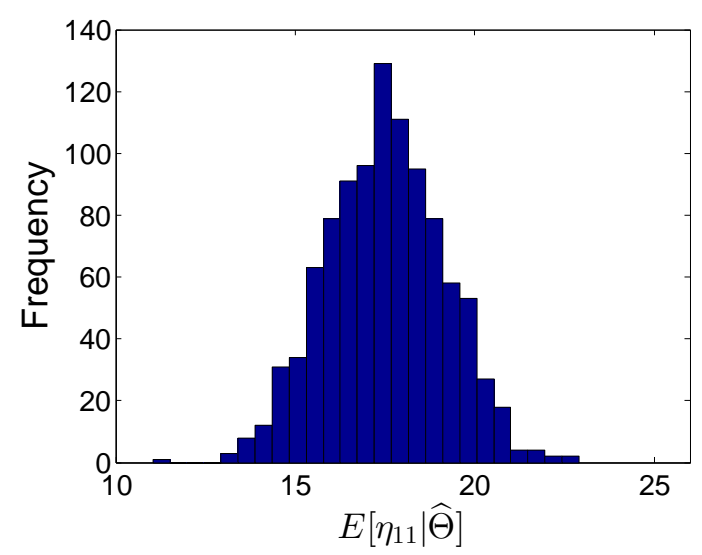

(e) Frequency of $\mathbb{E}\left(\eta_{11} \mid \widehat{\Theta}\right)$

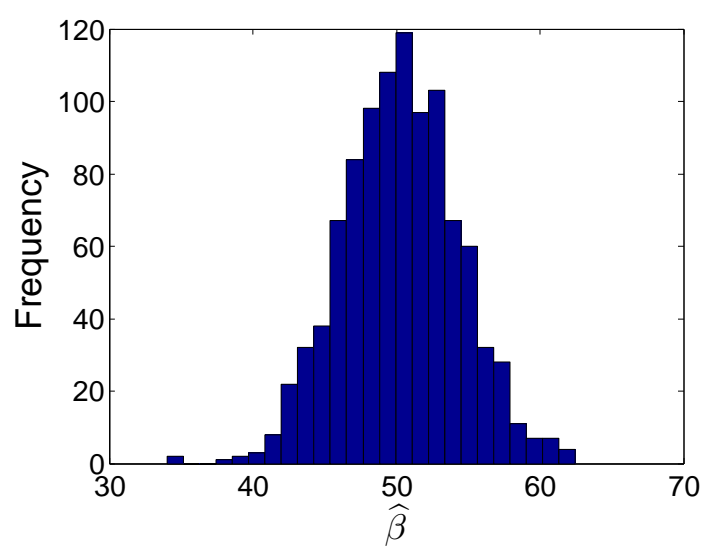

(b) Frequency of $\widehat{\beta}$

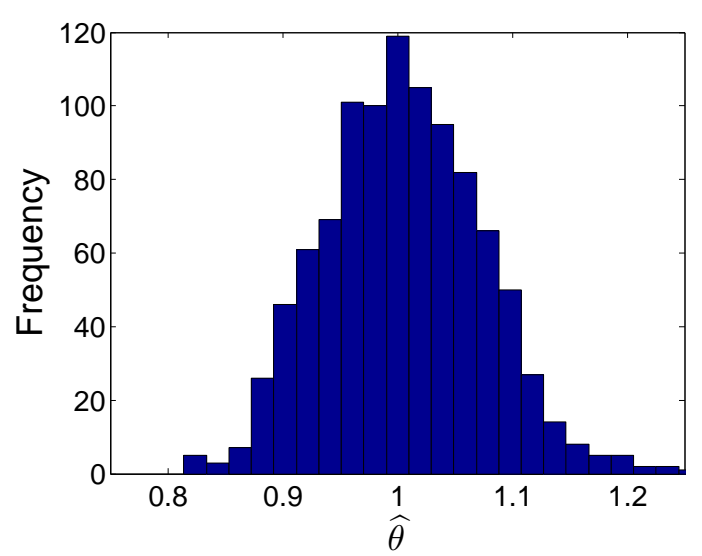

(d) Frequency of $\widehat{\theta}$

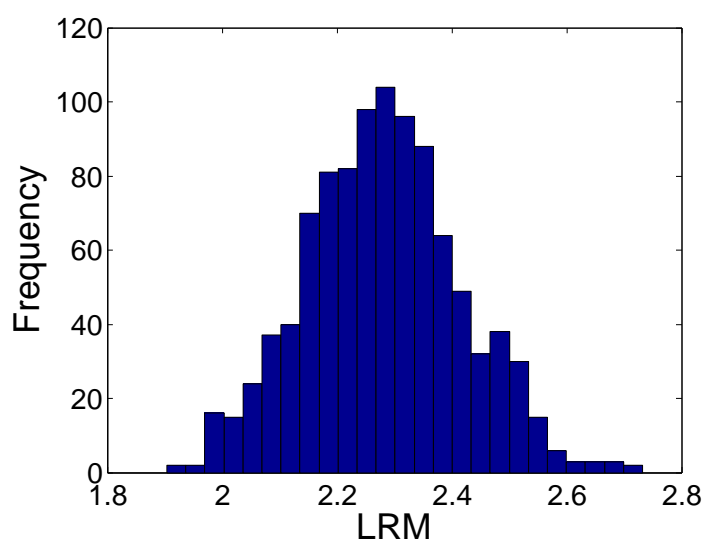

(f) Frequency of long run mean

Figure 5.3: Distribution of estimators (1,000 simulations of 2,000 data points) when $n=p=2, \boldsymbol{\eta}(0)=\mathbf{0}, \eta_{11}(1)=\eta_{22}(1), \eta_{12}(1)=\eta_{21}(1), \beta_{1}=\beta_{2}, \theta_{1}=\theta_{2}$, and using Algorithm I with $m=3$. 


\begin{tabular}{|c|c|c|c|c|c|c|c|c|c|}
\hline \multicolumn{10}{|c|}{$N=500$} \\
\hline & \multirow[t]{2}{*}{ true } & \multicolumn{2}{|c|}{$m=1$} & \multicolumn{2}{|c|}{$m=2$} & \multicolumn{2}{|c|}{$m=3$} & \multicolumn{2}{|c|}{$m=4$} \\
\hline & & mean & $(\operatorname{std})$ & mean & (std) & mean & (std) & mean & (std) \\
\hline$\widehat{\rho}$ & 0.7 & 0.768 & $(0.184)$ & 0.748 & $(0.179)$ & 0.748 & $(0.179)$ & 0.739 & $(0.172)$ \\
\hline$\widehat{\beta}_{1}$ & 50 & 52.78 & $(20.95)$ & 51.88 & $(14.30)$ & 51.26 & $(11.98)$ & 51.40 & (11.16) \\
\hline$\widehat{\beta}_{2}$ & 50 & 53.27 & $(17.96)$ & 51.21 & $(13.75)$ & 51.76 & $(12.33)$ & 50.93 & (10.62) \\
\hline$\widehat{\eta}_{11}(1)$ & 25 & 25.10 & $(12.02)$ & 25.63 & $(10.20)$ & 25.05 & $(9.01)$ & 25.30 & ( 8.37$)$ \\
\hline$\widehat{\eta}_{12}(1)$ & 15 & 14.91 & $(7.88)$ & 15.34 & $(6.84)$ & 15.51 & $(6.79)$ & 15.35 & $(5.70)$ \\
\hline$\widehat{\eta}_{21}(1)$ & 15 & 15.09 & $(8.22)$ & 15.26 & $(6.47)$ & 15.35 & $(6.14)$ & 15.37 & ( 5.76$)$ \\
\hline$\widehat{\eta}_{22}(1)$ & 15 & 25.60 & $(12.65)$ & 25.05 & $(9.50)$ & 25.07 & $(8.64)$ & 25.26 & $(8.15)$ \\
\hline$\widehat{\theta}_{1}$ & 1 & 0.998 & $(0.175)$ & 0.998 & $(0.147)$ & 1.008 & $(0.138)$ & 0.999 & $(0.133)$ \\
\hline$\widehat{\theta}_{2}$ & 1 & 1.001 & $(0.166)$ & 0.992 & $(0.147)$ & 1.007 & $(0.134)$ & 0.997 & $(0.126)$ \\
\hline $\mathbb{E}\left[\eta_{11} \mid \widehat{\Theta}\right]$ & 17.5 & 18.08 & $(6.98)$ & 18.09 & $(5.60)$ & 17.75 & $(4.88)$ & 17.85 & ( 4.61$)$ \\
\hline$L R M_{1}$ & 2.273 & 2.289 & $(0.277)$ & 2.310 & $(0.287)$ & 2.324 & $(0.288)$ & 2.300 & $(0.289)$ \\
\hline runtime & & 2.26 & $(1.13)$ & 1.98 & $(1.13)$ & 2.10 & $(1.28)$ & 2.50 & $(1.64)$ \\
\hline \multicolumn{10}{|c|}{$N=2,000$} \\
\hline$\widehat{\rho}$ & 0.7 & 0.720 & $(0.115)$ & 0.721 & $(0.105)$ & 0.712 & $(0.098)$ & 0.717 & $(0.095)$ \\
\hline$\widehat{\beta}_{1}$ & 50 & 50.30 & $(8.00)$ & 50.52 & $(6.50)$ & 50.53 & $(5.75)$ & 50.52 & ( 5.33$)$ \\
\hline$\widehat{\beta}_{2}$ & 50 & 50.72 & $(7.80)$ & 50.52 & $(6.48)$ & 50.37 & $(5.72)$ & 50.07 & (5.18) \\
\hline$\widehat{\eta}_{11}(1)$ & 25 & 24.96 & $(5.89)$ & 24.86 & $(4.83)$ & 25.21 & $(4.37)$ & 24.92 & ( 3.99$)$ \\
\hline$\widehat{\eta}_{12}(1)$ & 15 & 15.01 & $(3.87)$ & 15.05 & $(3.16)$ & 15.13 & $(2.94)$ & 15.05 & $(2.79)$ \\
\hline$\widehat{\eta}_{21}(1)$ & 15 & 15.12 & $(3.87)$ & 14.95 & $(3.22)$ & 15.07 & $(2.81)$ & 14.92 & ( 2.73$)$ \\
\hline$\widehat{\eta}_{22}(1)$ & 15 & 25.17 & $(5.82)$ & 24.83 & $(4.91)$ & 25.01 & $(4.31)$ & 24.77 & ( 4.08$)$ \\
\hline$\widehat{\theta}_{1}$ & 1 & 0.999 & $(0.083)$ & 0.996 & $(0.071)$ & 0.998 & $(0.066)$ & 1.000 & $(0.066)$ \\
\hline$\widehat{\theta}_{2}$ & 1 & 1.003 & $(0.081)$ & 1.002 & $(0.071)$ & 1.001 & $(0.066)$ & 1.001 & $(0.066)$ \\
\hline $\mathbb{E}\left[\eta_{11} \mid \widehat{\Theta}\right]$ & 17.5 & 17.55 & $(3.09)$ & 17.60 & $(2.56)$ & 17.68 & $(2.32)$ & 17.63 & ( 2.19) \\
\hline$L R M_{1}$ & 2.273 & 2.281 & $(0.140)$ & 2.273 & $(0.135)$ & 2.277 & $(0.134)$ & 2.281 & (0.135) \\
\hline runtime & & 3.25 & $(1.29)$ & 3.34 & $(1.48)$ & 4.73 & $(2.09)$ & 7.66 & $(3.34)$ \\
\hline \multicolumn{10}{|c|}{$N=10,000$} \\
\hline$\widehat{\rho}$ & 0.7 & 0.706 & $(0.051)$ & 0.704 & $(0.045)$ & 0.703 & $(0.044)$ & 0.703 & $(0.040)$ \\
\hline$\widehat{\beta}_{1}$ & 50 & 50.11 & ( 3.28$)$ & 50.26 & $(2.75)$ & 49.98 & $(2.59)$ & 50.15 & $(2.29)$ \\
\hline$\widehat{\beta}_{2}$ & 50 & 49.84 & $(3.34)$ & 50.02 & ( 2.67$)$ & 50.06 & $(2.51)$ & 50.10 & $(2.30)$ \\
\hline$\widehat{\eta}_{11}(1)$ & 25 & 24.86 & $(2.53)$ & 25.06 & $(2.08)$ & 24.94 & $(1.88)$ & 25.02 & ( 1.82$)$ \\
\hline$\widehat{\eta}_{12}(1)$ & 15 & 15.02 & ( 1.61$)$ & 15.05 & $(1.43)$ & 15.00 & $(1.28)$ & 14.99 & $(1.21)$ \\
\hline$\widehat{\eta}_{21}(1)$ & 15 & 14.86 & $(1.64)$ & 15.00 & $(1.43)$ & 15.00 & $(1.29)$ & 14.99 & $(1.22)$ \\
\hline$\widehat{\eta}_{22}(1)$ & 15 & 24.86 & $(2.60)$ & 24.90 & $(2.07)$ & 25.00 & $(2.02)$ & 24.99 & $(1.76)$ \\
\hline$\widehat{\theta}_{1}$ & 1 & 1.001 & $(0.037)$ & 1.000 & $(0.031)$ & 0.999 & $(0.031)$ & 1.000 & $(0.029)$ \\
\hline$\widehat{\theta}_{2}$ & 1 & 1.000 & $(0.037)$ & 1.001 & $(0.033)$ & 1.000 & $(0.030)$ & 1.000 & $(0.029)$ \\
\hline $\mathbb{E}\left[\eta_{11} \mid \widehat{\Theta}\right]$ & 17.5 & 17.46 & $(1.33)$ & 17.58 & ( 1.08$)$ & 17.47 & $(0.99)$ & 17.54 & $(0.94)$ \\
\hline$L R M_{1}$ & 2.273 & 2.274 & $(0.062)$ & 2.273 & $(0.059)$ & 2.272 & $(0.060)$ & 2.271 & $(0.062)$ \\
\hline runtime & & 7.21 & $(2.62)$ & 7.67 & $(2.82)$ & 13.60 & $(5.59)$ & 25.12 & $(11.20)$ \\
\hline
\end{tabular}

Table 5.3: Mean and standard deviations of estimators (1,000 simulations) when $n=$ $p=2$ and $\boldsymbol{\eta}(0)=\mathbf{0}$ and using Algorithm I. Total number of free parameters is 9 . 


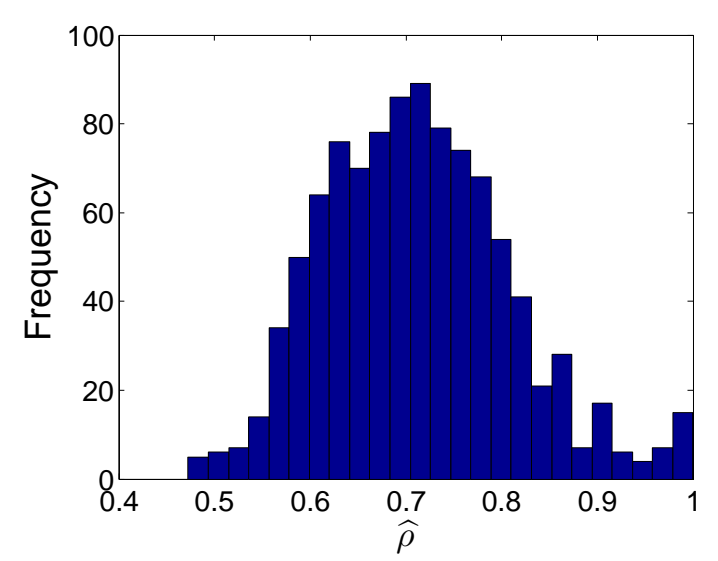

(a) Frequency of $\widehat{\rho}$

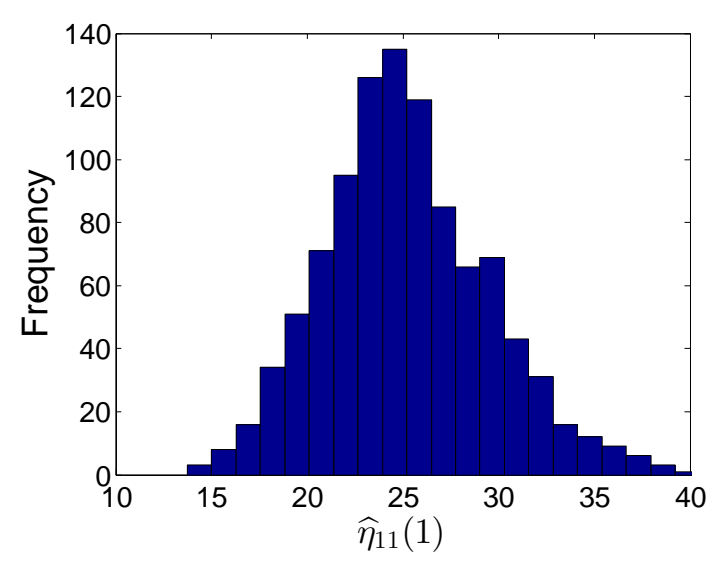

(c) Frequency of $\widehat{\eta}_{11}(1)$

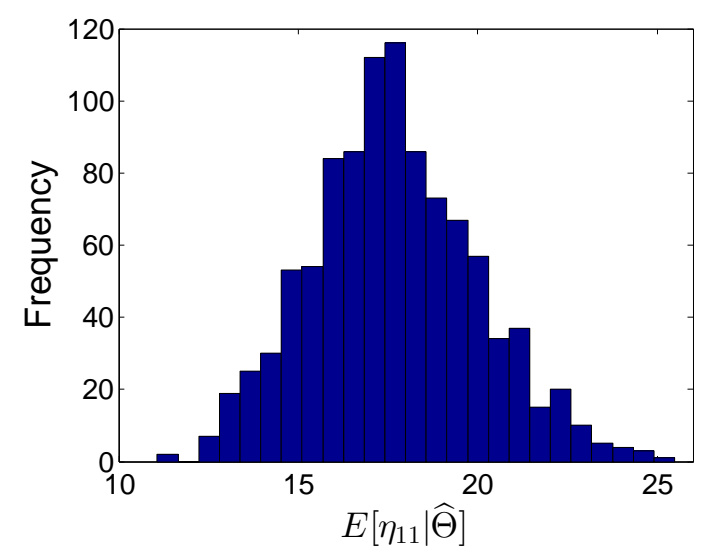

(e) Frequency of $\mathbb{E}\left(\eta_{11} \mid \widehat{\Theta}\right)$

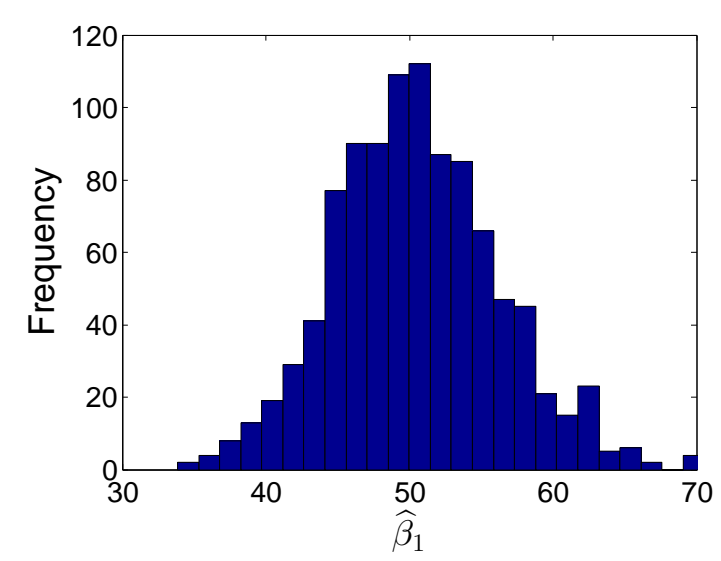

(b) Frequency of $\widehat{\beta}_{1}$

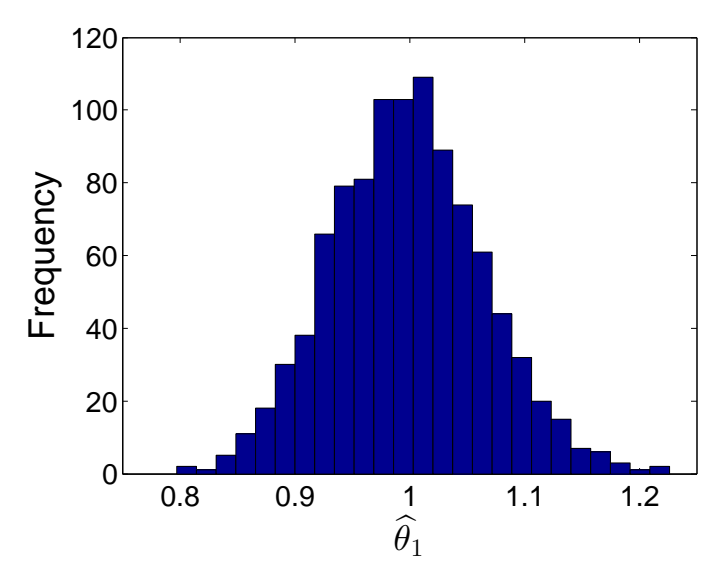

(d) Frequency of $\widehat{\theta}_{1}$

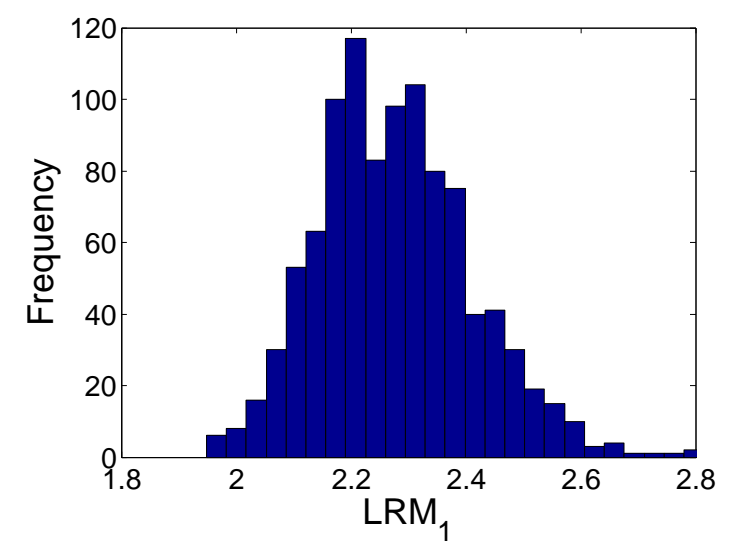

(f) Frequency of long run mean

Figure 5.4: Distribution of estimators (1,000 simulations of 2,000 data points) when $n=p=2, \boldsymbol{\eta}(0)=\mathbf{0}$, and using Algorithm I with $m=3$. This is the non-symmetric case. 
It is worthy to point out two things when considering the stated runtimes (which are stated in seconds). First, the machine that was used to perform these simulations is considerably old and is considered, by today's standards, obsolete. ${ }^{53}$ With this in mind, it is still useful for comparable relative performance between separate simulations. Secondly, the code is written in great generality, and can be optimized for specific cases by hardcoding dimensions and array sizes manually when speed is a priority (i.e. when deploying such an algorithm in practice). In terms of implementation specifics, MATLAB is the front end used for all calibrations with specific computations such as computing likelihood functions or simulating sample paths done in $\mathrm{C}++$ code compiled by MATLAB into .mexw64 files. If one is truely concerned with speed, making use of a numerical optimizer in $\mathrm{C}($ or $\mathrm{C}++)$ will offer a massive speed advantage over the MATLAB optimizer that is currently implemented.

To judge the stability of the fixed point search, we look at the tail of the distribution of the divergence difference of $\boldsymbol{\rho}$ and $\boldsymbol{\beta}$, denoted $\rho_{\mathrm{div}}$ and $\beta_{\mathrm{div}}$, respectively. If the algorithm breaks before the maximum number of iterations is reached, then $\rho_{\text {div }}$ and $\beta_{\text {div }}$ are both set to 0 . Otherwise, they are set to the maximum absolute difference between the fitted value and the value at the last 4 iterations. The $99^{\text {th }}$ percentile and the maximum value for 2,000 and 10,000 simulations are provided in Tables 5.4 and 5.5, respectively, for the symmetric case and Tables 5.6 and 5.7 for the non-symmetric case. Notice that increasing $m$ from 1 to 4 offers a reduction in size of the repeated oscillations of the diverging sequences $\rho_{k}$ and $\beta_{k}$ constructed in Section 5.3.2. Notice also that increasing the cluster size $m$ causes the algorithm to exit before the iteration count upper bound on more sample paths by observing the frequency at iteration count 29 in Figure 5.5. Both of these features suggest an increase in stability in the fixed point search.

Lastly, consulting Figure 5.5 justifies our choice of an upper bound of 9 iterations, as enforced in Step 6 of the calibration algorithm.

\footnotetext{
${ }^{53}$ Exact specifications include an Intel Pentium E5200 dual-core CPU (2 MB cache, $2.50 \mathrm{GHz}$ ) and 8 GB DDR3-1,066 MHz RAM.
} 


\begin{tabular}{|c|c|c|c|c|}
\hline & $m=1$ & $m=2$ & $m=3$ & $m=4$ \\
\hline $99^{\text {th }}$ perc. of $\rho_{\text {div }}$ & 0.0342 & 0.0199 & 0.0163 & 0.0129 \\
\hline maximum of $\rho_{\text {div }}$ & 0.0732 & 0.0399 & 0.0332 & 0.0264 \\
\hline $99^{\text {th }}$ perc. of $\beta_{1, \text { div }}$ & 1.6740 & 1.2490 & 0.8702 & 0.7803 \\
\hline maximum of $\beta_{1, \mathrm{div}}$ & 3.5301 & 2.2900 & 2.1060 & 1.1990 \\
\hline $99^{\text {th }}$ perc. of $\beta_{2, \text { div }}$ & 1.6740 & 1.2490 & 0.8702 & 0.7803 \\
\hline maximum of $\beta_{2, \mathrm{div}}$ & 3.5301 & 2.2900 & 2.1060 & 1.1990 \\
\hline
\end{tabular}

Table 5.4: Quantities associated with judging fixed point stability. 1,000 simulations of 2,000 points when $n=p=2, \boldsymbol{\eta}(0)=\mathbf{0}, \eta_{11}(1)=\eta_{22}(1), \eta_{12}(1)=\eta_{21}(1), \beta_{1}=\beta_{2}, \theta_{1}=\theta_{2}$, and using Algorithm I.

\begin{tabular}{rcccc}
\hline & $m=1$ & $m=2$ & $m=3$ & $m=4$ \\
\hline 99 $9^{\text {th }}$ perc. of $\rho_{\text {div }}$ & 0.0049 & 0.0042 & 0.0032 & 0.0026 \\
maximum of $\rho_{\text {div }}$ & 0.0091 & 0.0064 & 0.0057 & 0.0045 \\
99 $9^{\text {th }}$ perc. of $\beta_{1, \text { div }}$ & 0.3137 & 0.1939 & 0.1989 & 0.1574 \\
maximum of $\beta_{1, \text { div }}$ & 0.8332 & 0.3935 & 0.3799 & 0.2781 \\
99 $9^{\text {th }}$ perc. of $\beta_{2, \text { div }}$ & 0.3137 & 0.1939 & 0.1989 & 0.1574 \\
maximum of $\beta_{2, \text { div }}$ & 0.8332 & 0.3935 & 0.3799 & 0.2781 \\
\hline
\end{tabular}

Table 5.5: Quantities associated with judging fixed point stability. 1,000 simulations of 10,000 points when $n=p=2, \boldsymbol{\eta}(0)=\mathbf{0}, \eta_{11}(1)=\eta_{22}(1), \eta_{12}(1)=\eta_{21}(1), \beta_{1}=\beta_{2}$, $\theta_{1}=\theta_{2}$, and using Algorithm I.

\begin{tabular}{rcccc}
\hline & $m=1$ & $m=2$ & $m=3$ & $m=4$ \\
\hline 99 $9^{\text {th }}$ perc. of $\rho_{\text {div }}$ & 0.0342 & 0.0244 & 0.0160 & 0.0139 \\
maximum of $\rho_{\text {div }}$ & 0.0575 & 0.0439 & 0.0375 & 0.0282 \\
99 $9^{\text {th }}$ perc. of $\beta_{1, \text { div }}$ & 1.9714 & 1.7593 & 1.3430 & 1.4206 \\
maximum of $\beta_{1, \text { div }}$ & 4.6900 & 2.9871 & 2.3766 & 3.4232 \\
99 $9^{\text {th }}$ perc. of $\beta_{2, \text { div }}$ & 1.8483 & 1.4009 & 1.2609 & 1.1082 \\
maximum of $\beta_{2, \text { div }}$ & 6.9472 & 3.4182 & 2.0013 & 2.7650 \\
\hline
\end{tabular}

Table 5.6: Quantities associated with judging fixed point stability. 1,000 simulations of 2,000 points when $n=p=2, \boldsymbol{\eta}(0)=\mathbf{0}$, and using Algorithm I. This is the non-symmetric case. 


\begin{tabular}{rcccc}
\hline & $m=1$ & $m=2$ & $m=3$ & $m=4$ \\
\hline 99 $9^{\text {th }}$ perc. of $\rho_{\text {div }}$ & 0.0055 & 0.0041 & 0.0036 & 0.0032 \\
maximum of $\rho_{\text {div }}$ & 0.0099 & 0.0057 & 0.0064 & 0.0058 \\
99 $9^{\text {th }}$ perc. of $\beta_{1, \text { div }}$ & 0.3590 & 0.2643 & 0.2293 & 0.2200 \\
maximum of $\beta_{1, \text { div }}$ & 0.6788 & 0.4690 & 0.6056 & 0.4534 \\
99 $9^{\text {th }}$ perc. of $\beta_{2, \text { div }}$ & 0.3935 & 0.2902 & 0.2974 & 0.2382 \\
maximum of $\beta_{2, \text { div }}$ & 0.6788 & 0.5223 & 0.7381 & 0.3585 \\
\hline
\end{tabular}

Table 5.7: Quantities associated with judging fixed point stability. 1,000 simulations of 10,000 points when $n=p=2, \boldsymbol{\eta}(0)=\mathbf{0}$, and using Algorithm I. This is the nonsymmetric case.

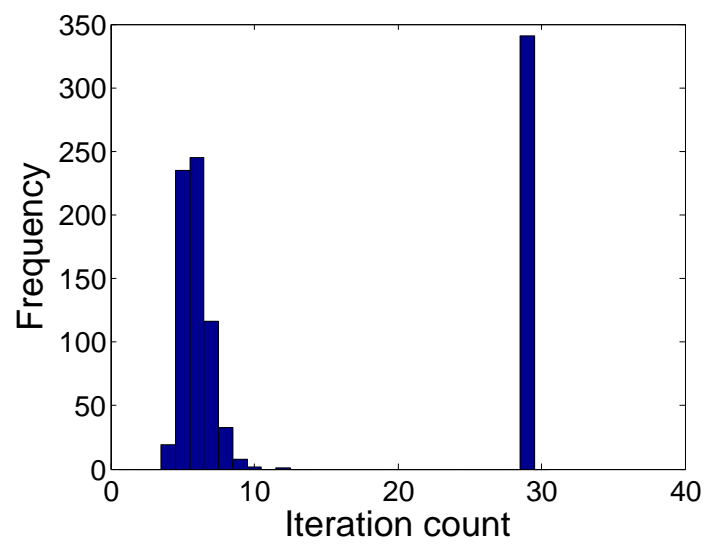

(a) $m=1$

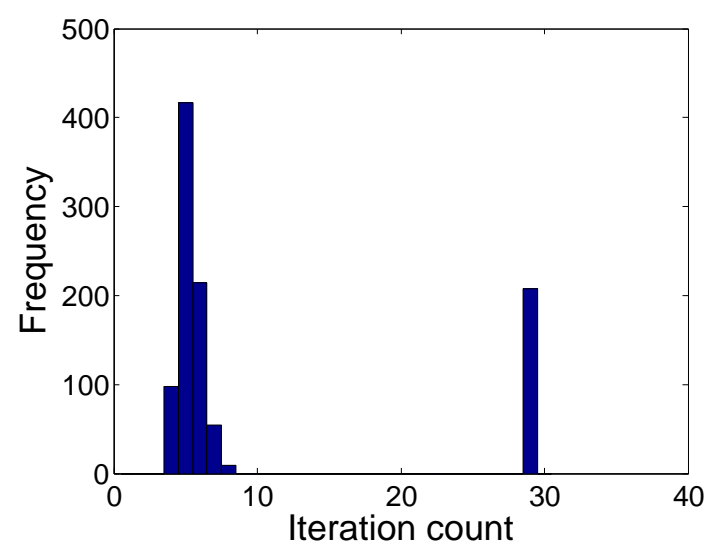

(c) $m=3$

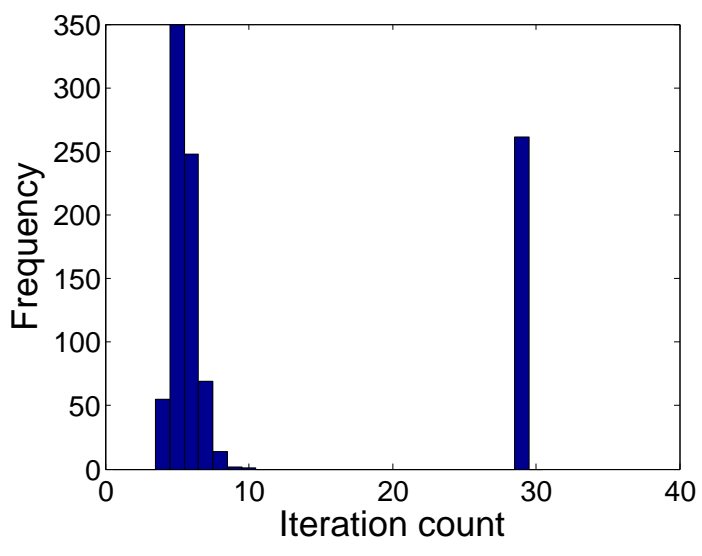

(b) $m=2$

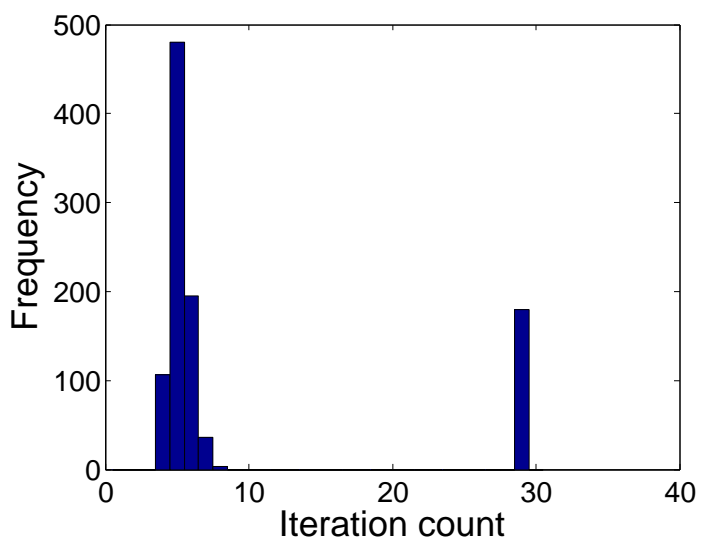

(d) $m=4$

Figure 5.5: Distribution for the number of iterations of the calibration algorithm on 1,000 simulations of 2,000 points in the symmetric model. 


\subsection{Calibration Algorithm II: Sequential Monte Carlo}

Calibration Algorithm I excels when $p=2$ and $\boldsymbol{\eta}(0)=\mathbf{0}$ with superior speed, but is not robust and fails to provide meaningful estimators when either $\boldsymbol{\eta}(0) \geq \mathbf{0}$ (interpreted element-wise) or $p>2$. To this end, we introduce a slower, yet more robust method of calibration that utilizes Sequential Monte Carlo particle filtering (see, e.g., Gordon et al. (1993), Pitt and Shephard (1999), and Doucet et al. (2000)) . These techniques will also provide a more reliable method to estimate $\boldsymbol{\lambda}_{t}$ online in Section 5.5.2.

\subsubsection{Sequential Monte Carlo \& Particle Filtering - Classical Method}

Here we briefly describe the SMC particle filtering algorithm for estimating the distribution of the hidden states $\boldsymbol{\lambda}_{t_{1}}, \ldots, \boldsymbol{\lambda}_{t_{N-1}}$, beginning with $M$ particles at $\boldsymbol{\lambda}_{0}=\boldsymbol{\theta}$.

At step $k \in\{2,3, \ldots, N\}$, we have $\boldsymbol{\lambda}_{t_{k-2}}$ and wish to propagate particles $i=1, \ldots, M$ forward to get $\boldsymbol{\lambda}_{t_{k-1}}$.

1. Draw $M$ samples of $\boldsymbol{\lambda}_{t_{k-1}}$ from the posterior distribution

$$
\boldsymbol{\lambda}_{t_{k-1}}^{(i)} \sim \mathbb{P}\left[\boldsymbol{\lambda}_{t_{k-1}} \mid \boldsymbol{\lambda}_{t_{k-2}}, T_{k-1}, T_{k}\right]
$$

(a) compute $l_{k-1}(q):=l\left(q \mid \boldsymbol{\lambda}_{t_{k-2}}, T_{k-1}, T_{k}\right)$ (i.e. probability that $H_{k-1}=q$, for $q=1, \ldots, p-1$, given $\boldsymbol{\lambda}_{t_{n-2}}, T_{n-1}$, and $\left.T_{n}\right)$ where $l\left(q \mid y, S_{1}, S_{2}\right)$ is provided in Observation 5.2.4.

(b) Draw iid uniforms $u^{(i)} \sim U(0,1)$ for each $i=1, \ldots, M$

(c) Use the uniform $u^{(i)}$ to simulate and set $H_{k-1}^{(i)}=q \in\{0,1, \ldots, p-1\}$ with corresponding probability $l_{k-1}(q)$. That is, set

$$
H_{k-1}^{(i)}=\sup \left\{q \in\{0,1, \ldots, p-1\}: \sum_{z=0}^{q-1} l_{k-1}(z) \leq u^{(i)}\right\}
$$

(d) Set

$$
\boldsymbol{\lambda}_{t_{k-1}}^{(i)}=\boldsymbol{\theta}+e^{-\boldsymbol{\beta} T_{k-1}(1)}\left(\boldsymbol{\lambda}_{t_{k-2}}^{(i)}-\boldsymbol{\theta}\right)+\boldsymbol{\eta}\left(H_{k-1}^{(i)}\right) e_{T_{k-1}(2)}
$$


2. Compute the un-normalized weights, given the observation $T_{k}$

$$
\begin{aligned}
\alpha_{k}^{(i)} & =\frac{\mathbb{P}\left[T_{k} \mid \boldsymbol{\lambda}_{t_{k-1}}=\boldsymbol{\lambda}_{t_{k-1}}^{(i)}\right] \mathbb{P}\left[H_{k-1}=H_{k-1}^{(i)} \mid \boldsymbol{\lambda}_{t_{k-2}}=\boldsymbol{\lambda}_{t_{k-2}}^{(i)}, T_{k-1}\right]}{\mathbb{P}\left[H_{k-1}=H_{k-1}^{(i)} \mid \boldsymbol{\lambda}_{t_{k-2}}=\boldsymbol{\lambda}_{t_{k-2}}^{(i)}, T_{k-1}, T_{k}\right]} \\
& =g\left(T_{k}, H_{k-1}^{(i)} \mid \boldsymbol{\lambda}_{t_{k-1}}^{(i)}\right) \times \frac{\rho_{H_{k-1}^{(i)}}}{l_{k-1}\left(H_{k-1}^{(i)}\right)}
\end{aligned}
$$

where $g(t, l \mid y)$ is provided by Equation (5.6) in Observation 5.2.3.

3. Compute and resample from the normalized weights.

(a) $W_{k}^{(i)}=\alpha_{k}^{(i)} / \sum_{j} \alpha_{k}^{(j)}$

(b) Draw a single uniform $u \sim U(0,1)$ and define $u_{i}=\frac{u+i-1}{M}, i=1, \ldots, M$

(c) Find $N_{i}$ such that $\sum_{j=1}^{N_{i}-1} W_{k}^{(j)} \leq u_{i} \leq \sum_{j=1}^{N_{i}} W_{k}^{(j)}$. The number of "offspring" for particle $i$ is given by $M_{i}=N_{i}-N_{i-1}$ (with $N_{0}=0$ )

(d) For each $\boldsymbol{\lambda}_{t_{k-1}}^{(i)}$, create $M_{i}$ offspring and call these $\overline{\boldsymbol{\lambda}}_{t_{k-1}}^{(i)}$

(e) The updated equally weighted paths of the particles are $\overline{\boldsymbol{\lambda}}_{t_{1}}^{(i)}, \ldots, \overline{\boldsymbol{\lambda}}_{t_{k-1}}^{(i)}$

\subsubsection{The Calibration Algorithm}

Similar to the composite likelihood framework of Section 5.3.4, we split up the density into the product of conditional densities ${ }^{54}$ (instead of marginal densities as was done previously) via

$$
\mathcal{L}(\Theta ; y)=f\left(z_{1} ; \Theta\right) \prod_{k \geq 2} f\left(z_{k} ; z_{1}, \ldots, z_{k-1}, \Theta\right),
$$

where $f\left(z_{k} ; \cdot, \Theta\right)$ is the conditional density of the $k^{\text {th }}$ block of data.

As done in Algorithm I, the simpler $\boldsymbol{\rho}=1$ model is calibrated first. While constraining the long run mean to remain unchanged, we proceed to approximate each factor in $f\left(z_{1} ; \Theta\right) \prod_{k=2}^{N} f\left(z_{k} ; z_{1}, \ldots, z_{k-1}, \Theta\right)$ by particle propagation to estimate the distribution of $\boldsymbol{\lambda}_{t_{k-1}}$. More specifically,

1. Propagate the particles to $t_{k-2}$ as outlined in Section 5.4.1.

2. Decay $\boldsymbol{\lambda}_{t}$ by rate matrix $\boldsymbol{\beta}$ to $t_{k-1}$.

\footnotetext{
${ }^{54}$ For composite conditional likelihood methods, see Stein et al. (2004).
} 
3. Add a randomly generated jump $\boldsymbol{\eta}$ from the prior jump distribution (i.e. the distribution with probabilities given by $\boldsymbol{\rho}$ ).

The explicit details for estimating $\boldsymbol{\lambda}_{t}$ for all $t$ (i.e. not just at event times $t_{k}$ ) are given in Section 5.5.2.

\subsubsection{Numerical Results on Simulated Data: $\boldsymbol{\eta}(0)=0$}

See Table 5.8 and 5.9 for the means and standard deviations of the estimators using Algorithm II performed on the two models that were calibrated in Section 5.3.5. Observe that there is a noticable increase in accuracy at the cost of runtime, especially for $N=500$ data points. Notice that the reduction in the estimators' standard errors over Algorithm I (across all cluster sizes $m=1,2,3,4$ ) exists even for as few as 10 particles. Figures 5.6 and 5.7 depict the distributions of the aforementioned estimators for $N=2,000$ data points and using 20 particles. 


\begin{tabular}{|c|c|c|c|c|c|c|c|}
\hline \multicolumn{8}{|c|}{$N=500$} \\
\hline & \multirow[t]{2}{*}{ true } & \multicolumn{2}{|c|}{10 particles } & \multicolumn{2}{|c|}{20 particles } & \multicolumn{2}{|c|}{50 particles } \\
\hline & & mean & (std) & mean & (std) & mean & (std) \\
\hline$\widehat{\rho}$ & 0.7 & 0.690 & $(0.153)$ & 0.690 & $(0.155)$ & 0.684 & $(0.157)$ \\
\hline$\widehat{\beta}$ & 50 & 51.93 & $(5.49)$ & 51.76 & $(5.45)$ & 51.38 & $(5.46)$ \\
\hline$\widehat{\eta}_{11}(1)$ & 25 & 27.25 & $(6.15)$ & 27.06 & $(6.05)$ & 27.36 & $(6.40)$ \\
\hline$\widehat{\eta}_{12}(1)$ & 15 & 16.32 & $(3.99)$ & 16.25 & $(3.85)$ & 16.28 & ( 4.03$)$ \\
\hline$\widehat{\theta}$ & 1 & 1.016 & $(0.131)$ & 1.015 & $(0.124)$ & 1.019 & (0.128) \\
\hline $\mathbb{E}\left[\eta_{11} \mid \widehat{\Theta}\right]$ & 17.5 & 18.07 & $(2.51)$ & 17.91 & $(2.46)$ & 17.90 & $(2.41)$ \\
\hline$L R M$ & 2.273 & 2.310 & $(0.287)$ & 2.299 & $(0.282)$ & 2.312 & $(0.287)$ \\
\hline runtime & & 4.42 & $(2.72)$ & 8.68 & ( 6.88$)$ & 21.45 & (14.98) \\
\hline \multicolumn{8}{|c|}{$N=2,000$} \\
\hline$\widehat{\rho}$ & 0.7 & 0.717 & $(0.087)$ & 0.706 & $(0.089)$ & 0.710 & $(0.090)$ \\
\hline$\widehat{\beta}$ & 50 & 50.84 & $(2.73)$ & 50.66 & $(2.79)$ & 50.64 & $(2.72)$ \\
\hline$\widehat{\eta}_{11}(1)$ & 25 & 24.96 & $(2.86)$ & 25.39 & $(3.08)$ & 25.24 & ( 3.12$)$ \\
\hline$\widehat{\eta}_{12}(1)$ & 15 & 15.04 & $(1.82)$ & 15.19 & $(1.85)$ & 15.18 & ( 1.91$)$ \\
\hline$\widehat{\theta}$ & 1 & 1.004 & $(0.066)$ & 1.006 & $(0.064)$ & 1.004 & $(0.061)$ \\
\hline $\mathbb{E}\left[\eta_{11} \mid \widehat{\Theta}\right]$ & 17.5 & 17.70 & $(1.22)$ & 17.69 & $(1.24)$ & 17.69 & $(1.24)$ \\
\hline$L R M$ & 2.273 & 2.277 & $(0.139)$ & 2.279 & $(0.139)$ & 2.283 & $(0.140)$ \\
\hline runtime & & 17.64 & $(31.90)$ & 31.20 & $(23.78)$ & 89.48 & (115.73) \\
\hline
\end{tabular}

Table 5.8: Mean and standard deviations of estimators (1,000 simulations) when $n=$ $p=2, \boldsymbol{\eta}(0)=\mathbf{0}, \eta_{11}(1)=\eta_{22}(1), \eta_{12}(1)=\eta_{21}(1), \beta_{1}=\beta_{2}, \theta_{1}=\theta_{2}$, and using Algorithm II. Total number of free parameters is 5 . 


\begin{tabular}{|c|c|c|c|c|c|c|c|}
\hline \multicolumn{8}{|c|}{$N=500$} \\
\hline & \multirow[t]{2}{*}{ true } & \multicolumn{2}{|c|}{10 particles } & \multicolumn{2}{|c|}{20 particles } & \multicolumn{2}{|c|}{50 particles } \\
\hline & & mean & (std) & mean & (std) & mean & (std) \\
\hline$\widehat{\rho}$ & 0.7 & 0.667 & $(0.154)$ & 0.676 & $(0.159)$ & 0.689 & $(0.168)$ \\
\hline$\widehat{\widehat{\beta}}_{1}$ & 50 & 52.20 & $(7.71)$ & 52.07 & $(7.40)$ & 52.22 & $(7.79)$ \\
\hline$\widehat{\beta}_{2}$ & 50 & 52.13 & $(7.73)$ & 52.01 & $(7.99)$ & 51.97 & $(7.54)$ \\
\hline$\widehat{\eta}_{11}(1)$ & 25 & 28.17 & $(7.46)$ & 27.94 & $(7.53)$ & 27.41 & $(7.72)$ \\
\hline$\widehat{\eta}_{12}(1)$ & 15 & 17.11 & $(5.12)$ & 16.90 & $(5.22)$ & 16.80 & $(5.14)$ \\
\hline$\widehat{\eta}_{21}(1)$ & 15 & 17.30 & $(5.16)$ & 16.96 & $(4.98)$ & 16.99 & $(5.16)$ \\
\hline$\widehat{\eta}_{22}(1)$ & 15 & 28.04 & $(7.36)$ & 27.75 & $(7.44)$ & 27.39 & $(7.60)$ \\
\hline$\widehat{\theta}_{1}$ & 1 & 1.011 & $(0.103)$ & 1.011 & $(0.107)$ & 1.009 & $(0.110)$ \\
\hline$\widehat{\theta}_{2}$ & 1 & 1.011 & $(0.106)$ & 1.012 & $(0.111)$ & 1.011 & $(0.104)$ \\
\hline $\mathbb{E}\left[\eta_{11} \mid \widehat{\Theta}\right]$ & 17.5 & 17.99 & $(3.53)$ & 18.05 & $(3.38)$ & 17.97 & $(3.48)$ \\
\hline$L R M_{1}$ & 2.273 & 2.293 & $(0.277)$ & 2.303 & $(0.283)$ & 2.303 & $(0.288)$ \\
\hline runtime & & 4.48 & $(3.10)$ & 8.26 & $(5.30)$ & 21.39 & (13.68) \\
\hline \multicolumn{8}{|c|}{$N=2,000$} \\
\hline$\widehat{\rho}$ & 0.7 & 0.714 & $(0.090)$ & 0.707 & $(0.088)$ & 0.705 & $(0.089)$ \\
\hline$\widehat{\beta}_{1}$ & 50 & 50.95 & ( 3.96$)$ & 50.33 & $(3.72)$ & 50.56 & $(4.00)$ \\
\hline$\widehat{\beta}_{2}$ & 50 & 51.06 & $(3.92)$ & 50.67 & $(3.85)$ & 50.58 & $(3.90)$ \\
\hline$\widehat{\eta}_{11}(1)$ & 25 & 25.21 & $(3.67)$ & 25.16 & $(3.49)$ & 25.34 & ( 3.43$)$ \\
\hline$\widehat{\eta}_{12}(1)$ & 15 & 15.17 & $(2.31)$ & 15.12 & $(2.18)$ & 15.18 & ( 2.19) \\
\hline$\widehat{\eta}_{21}(1)$ & 15 & 15.28 & $(2.37)$ & 15.21 & $(2.28)$ & 15.14 & ( 2.19) \\
\hline$\widehat{\eta}_{22}(1)$ & 15 & 25.30 & $(3.62)$ & 25.41 & $(3.53)$ & 25.40 & $(3.43)$ \\
\hline$\widehat{\theta}_{1}$ & 1 & 1.004 & $(0.054)$ & 0.999 & $(0.054)$ & 1.002 & $(0.053)$ \\
\hline$\widehat{\theta}_{2}$ & 1 & 1.003 & $(0.053)$ & 1.002 & $(0.052)$ & 1.002 & $(0.055)$ \\
\hline $\mathbb{E}\left[\eta_{11} \mid \widehat{\Theta}\right]$ & 17.5 & 17.75 & $(1.78)$ & 17.57 & $(1.67)$ & 17.65 & $(1.73)$ \\
\hline$L R M_{1}$ & 2.273 & 2.281 & $(0.141)$ & 2.275 & $(0.140)$ & 2.276 & $(0.140)$ \\
\hline runtime & & 15.79 & $(14.56)$ & 30.19 & $(23.03)$ & 75.56 & $(56.66)$ \\
\hline
\end{tabular}

Table 5.9: Mean and standard deviations of estimators (1,000 simulations) when $n=$ $p=2, \boldsymbol{\eta}(0)=\mathbf{0}$, and using Algorithm II. Total number of free parameters is 9 . 


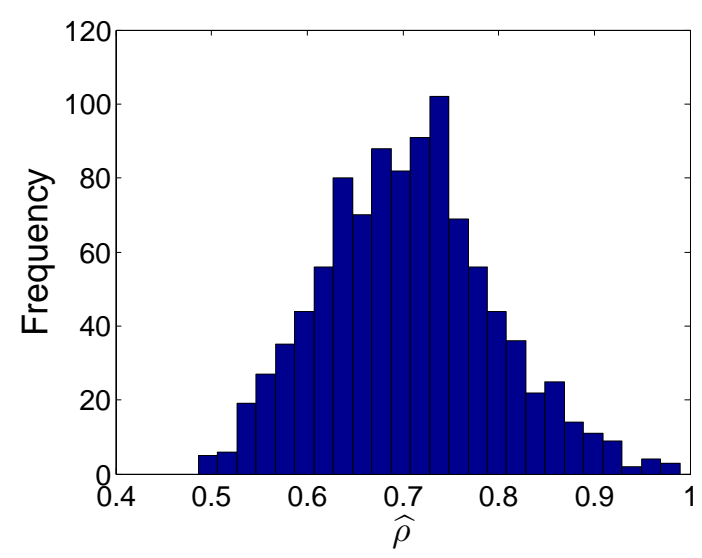

(a) Frequency of $\widehat{\rho}$

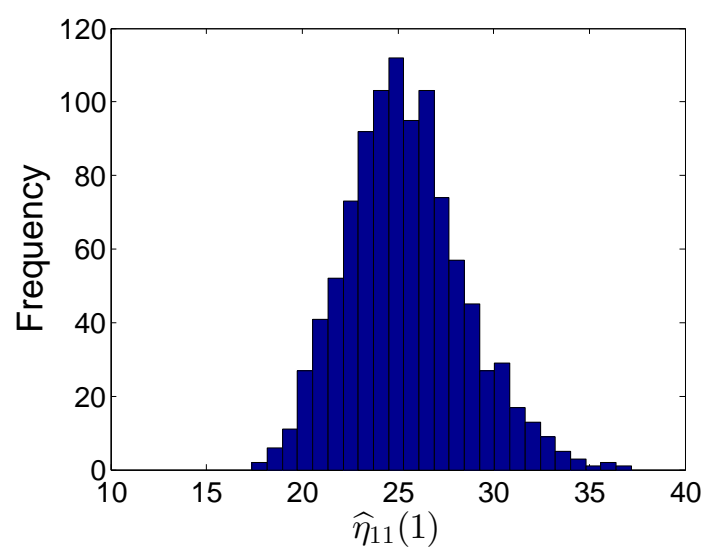

(c) Frequency of $\widehat{\eta}_{11}(1)$

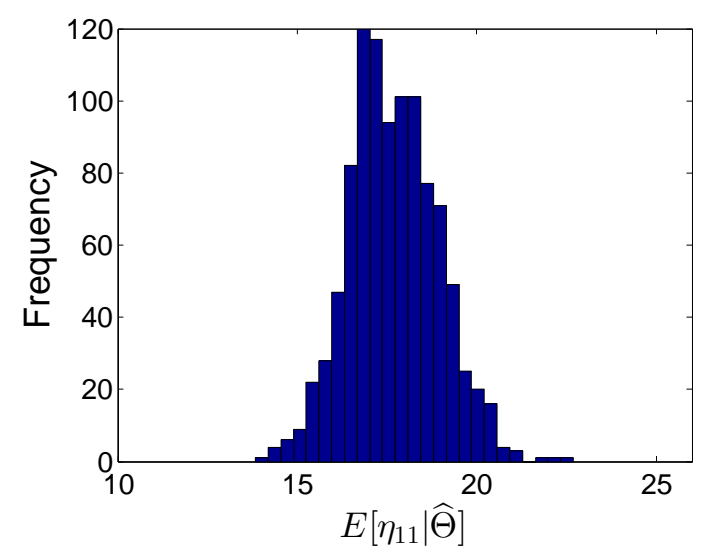

(e) Frequency of $\mathbb{E}\left(\eta_{11} \mid \widehat{\Theta}\right)$

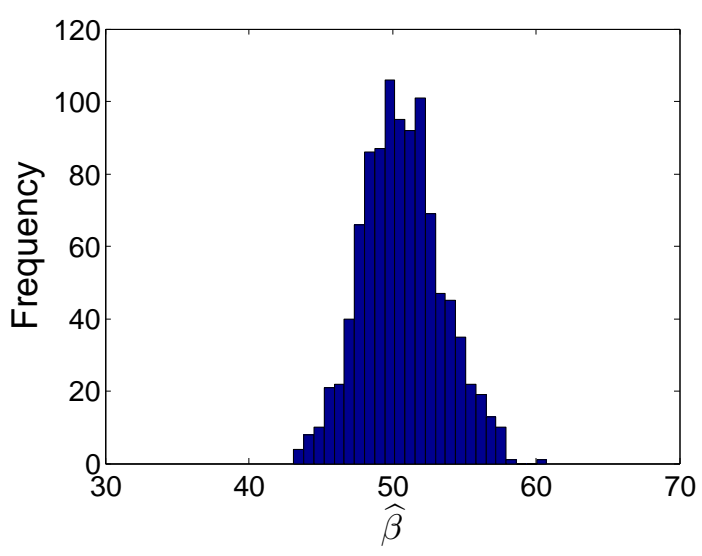

(b) Frequency of $\widehat{\beta}$

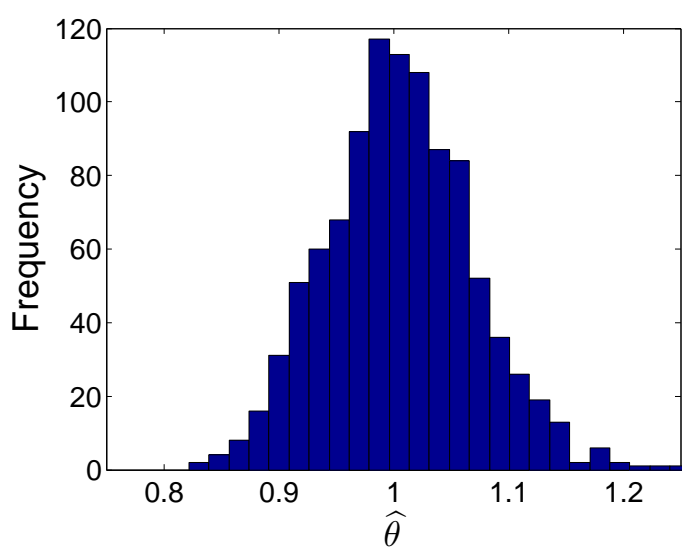

(d) Frequency of $\widehat{\theta}$

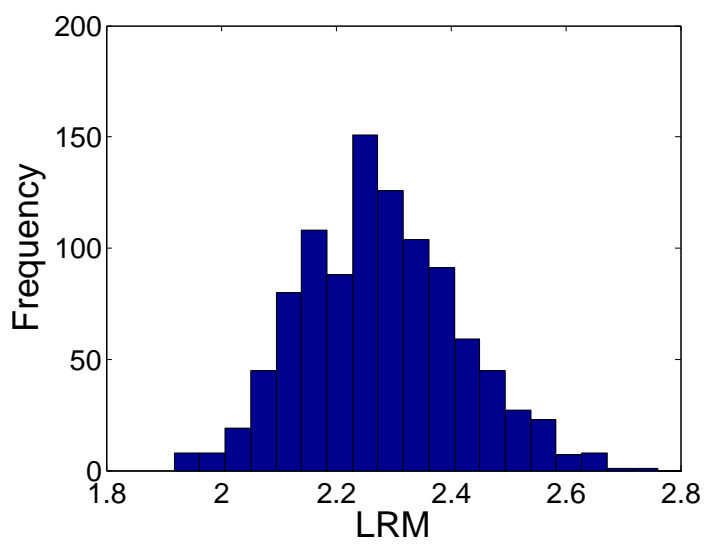

(f) Frequency of long run mean

Figure 5.6: Distribution of estimators (1,000 simulations of 2,000 data points) when $n=p=2, \boldsymbol{\eta}(0)=\mathbf{0}, \eta_{11}(1)=\eta_{22}(1), \eta_{12}(1)=\eta_{21}(1), \beta_{1}=\beta_{2}, \theta_{1}=\theta_{2}$, and using Algorithm II with 20 particles. 


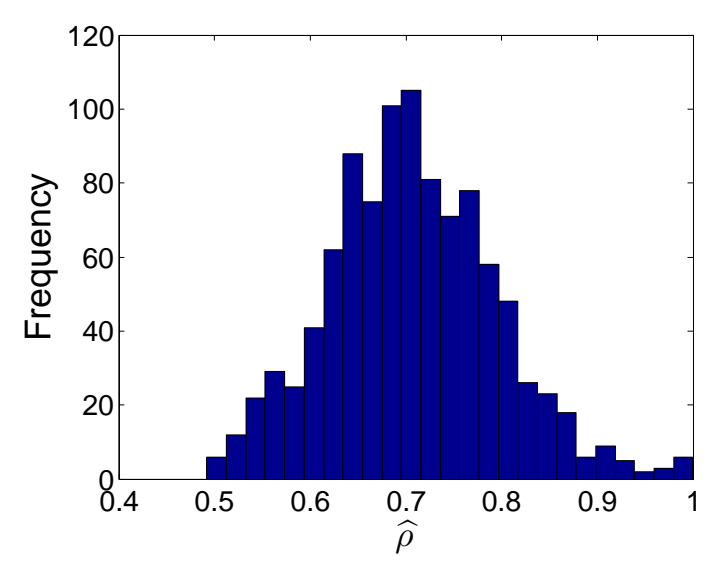

(a) Frequency of $\widehat{\rho}$

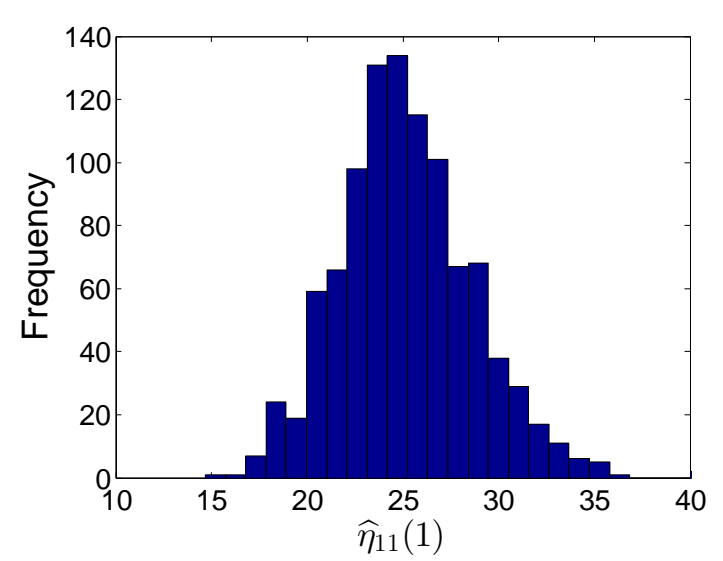

(c) Frequency of $\widehat{\eta}_{11}(1)$

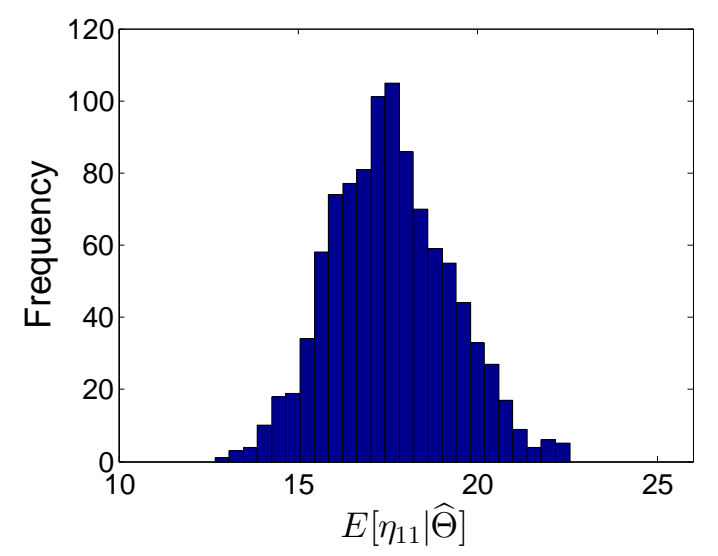

(e) Frequency of $\mathbb{E}\left(\eta_{11} \mid \widehat{\Theta}\right)$

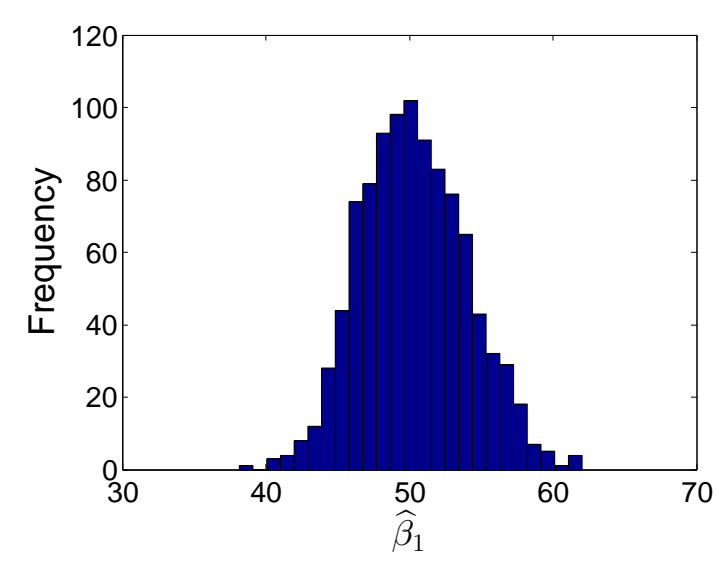

(b) Frequency of $\widehat{\beta}_{1}$

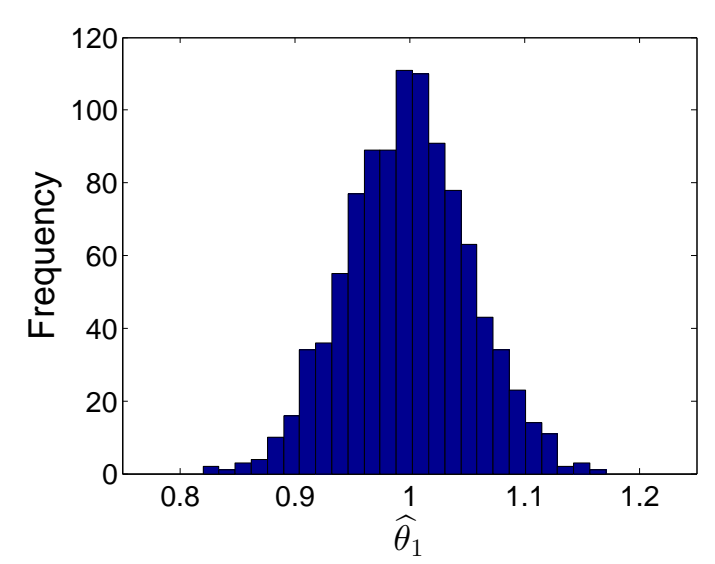

(d) Frequency of $\widehat{\theta}_{1}$

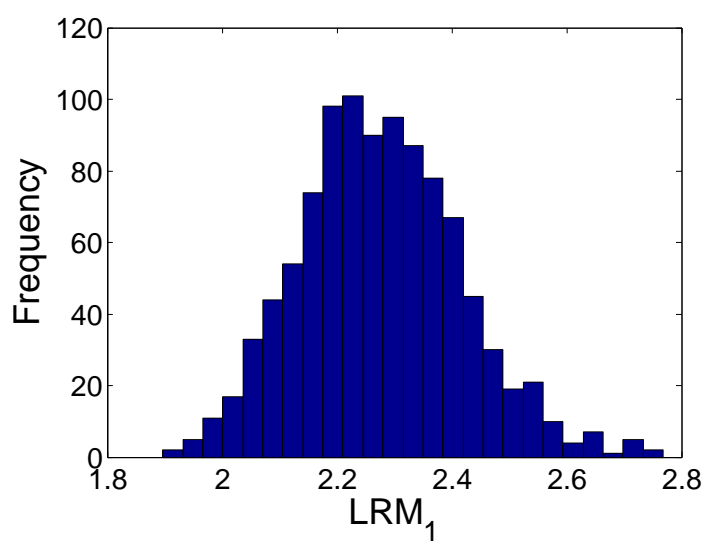

(f) Frequency of long run mean

Figure 5.7: Distribution of estimators (1,000 simulations of 2,000 data points) when $n=p=2, \boldsymbol{\eta}(0)=\mathbf{0}$, and using Algorithm II with 20 particles. This is the non-symmetric case. 


\subsubsection{Numerical Results on Simulated Data: $\boldsymbol{\eta}(0) \geq 0$}

As previously mentioned, Algorithm I breaks down when the $\boldsymbol{\eta}(0)=\mathbf{0}$ constraint is relaxed to $\boldsymbol{\eta}(0) \geq \mathbf{0}$ and does not yield meaningful parameter estimates. This simulation study reveals that Algorithm II is robust enough to provide reasonable parameter estimates in this more generalized setting. Although the runtime is significantly longer than its $\boldsymbol{\eta}(0)=\mathbf{0}$ counterpart (due to the fact that more particles are required to better approximate the posterior distribution of $\boldsymbol{\lambda}_{t}$ ), it is still far faster than currently known Monte Carle Expectation Maximization (MCEM) algorithms. In terms of running this in a high frequency trading framework, one can run the calibration in a parallel process and rely on real-time online updating as discussed in Section 5.5. The observed runtime can allow re-calibration of parameters as often as every few minutes on a reasonably powered machine and is a feasable solution in a variety of practical settings.

See Tables 5.10 and 5.11 for means and standard errors of estimators and other quantities of interest. Figures 5.8 and 5.9 also provide the histograms of the distributions of some of these statistics. Observe that there is much more variability in the estimators of $\boldsymbol{\eta}(0)$ and $\boldsymbol{\eta}(1)$ (and $\rho$ as well). However, in spite of this, the estimates of the mean jump size $\mathbb{E}[\boldsymbol{\eta}]$ still have relatively low standard deviation. The algorithm also detects the long run mean with reasonable accuracy. It is difficult to differentiate two points in the parameter space that have equal long run mean and mean jump size as the difference between the likelihood function is extremely small and influenced by noise.

Given this algorithm's ability to identify long run mean, mean jump size, and extract reasonable and meaningful parameter estimates while keeping run-time down to a few minutes, it is clear that it is a useful complimentary method to Algorithm I that performs well for small samples and/or when $\boldsymbol{\eta}(0) \geq \mathbf{0}$. 


\begin{tabular}{|c|c|c|c|c|c|}
\hline \multicolumn{6}{|c|}{$N=2,000$} \\
\hline & \multirow[t]{2}{*}{ true } & \multicolumn{2}{|c|}{50 particles } & \multicolumn{2}{|c|}{200 particles } \\
\hline & & mean & (std) & mean & (std) \\
\hline$\widehat{\rho}$ & 0.7 & 0.572 & $(0.147)$ & 0.592 & $(0.136)$ \\
\hline$\widehat{\beta}$ & 50 & 49.81 & $(2.70)$ & 49.91 & $(2.76)$ \\
\hline$\widehat{\eta}_{11}(0)$ & 5 & 8.07 & ( 3 & 7.06 & $(3.34)$ \\
\hline$\widehat{\eta}_{11}(1)$ & 25 & 27.64 & $(5.10)$ & 27.41 & ( 4.67$)$ \\
\hline$\widehat{\eta}_{12}(0)$ & 5 & 7.03 & $(2$ & 6.65 & $(2.43)$ \\
\hline$\widehat{\eta}_{12}(1)$ & 15 & 16.20 & $(2.96)$ & 16.19 & $(2.84)$ \\
\hline$\widehat{\theta}$ & 1 & 1.007 & $(0.057)$ & 0.997 & (0.061) \\
\hline $\mathbb{E}\left[\eta_{11} \mid \widehat{\Theta}\right]$ & 19 & 18.82 & $(1.43)$ & 18.88 & $(1.30)$ \\
\hline $\mathbb{E}\left[\eta_{12} \mid \widehat{\Theta}\right]$ & 12 & 11.99 & $(0.91)$ & 12.03 & ( 1.04$)$ \\
\hline$L R M$ & 2.632 & 2.643 & $(0.1$ & 2.630 & (0.191) \\
\hline runtime & & 72.49 & (48.96) & 382.85 & $(317.60)$ \\
\hline
\end{tabular}

Table 5.10: Mean and standard deviations of estimators (250 simulations) when $n=$ $p=2, \eta_{11}(1)=\eta_{22}(1), \eta_{12}(1)=\eta_{21}(1), \beta_{1}=\beta_{2}, \theta_{1}=\theta_{2}$, and using Algorithm II. Total number of free parameters is 7 . 


\begin{tabular}{|c|c|c|c|c|c|}
\hline \multicolumn{6}{|c|}{$N=2,000$} \\
\hline & \multirow[t]{2}{*}{ true } & \multicolumn{2}{|c|}{50 particles } & \multicolumn{2}{|c|}{200 particles } \\
\hline & & mean & (std) & mean & (std) \\
\hline$\widehat{\rho}$ & 0.7 & 0.589 & $(0.101)$ & 0.595 & $(0.102)$ \\
\hline$\widehat{\beta}_{1}$ & 50 & 49.86 & $(3.21)$ & 49.95 & ( 3.62$)$ \\
\hline$\widehat{\beta}_{2}$ & 50 & 50.24 & $(3.47)$ & 49.83 & ( 3.59$)$ \\
\hline$\widehat{\eta}_{11}(0)$ & 5 & 7.79 & $(3.20)$ & 7.34 & ( 3.54$)$ \\
\hline$\widehat{\eta}_{11}(1)$ & 25 & 27.22 & $(4.26)$ & 27.18 & $(4.44)$ \\
\hline$\widehat{\eta}_{12}(0)$ & 5 & 7.11 & $(2.67)$ & 6.16 & ( 2.71$)$ \\
\hline$\widehat{\eta}_{12}(1)$ & 15 & 15.81 & $(3.22)$ & 16.60 & ( 3.58$)$ \\
\hline$\widehat{\eta}_{21}(0)$ & 5 & 6.78 & $(2.91)$ & 5.93 & $(2.74)$ \\
\hline$\widehat{\eta}_{21}(1)$ & 15 & 16.06 & $(3.45)$ & 16.47 & (3.05) \\
\hline$\widehat{\eta}_{22}(0)$ & 5 & 8.27 & $(3.63)$ & 7.20 & (3.18) \\
\hline$\widehat{\eta}_{22}(1)$ & 25 & 27.01 & $(4.34)$ & 27.22 & $(4.23)$ \\
\hline$\widehat{\theta}_{1}$ & 1 & 1.000 & $(0.057)$ & 1.005 & $(0.056)$ \\
\hline$\widehat{\theta}_{2}$ & 1 & 1.008 & $(0.061)$ & 1.001 & $(0.056)$ \\
\hline $\mathbb{E}\left[\eta_{11} \mid \widehat{\Theta}\right]$ & 19 & 18.96 & $(1.66)$ & 18.87 & $(1.65)$ \\
\hline $\mathbb{E}\left[\eta_{12} \mid \widehat{\Theta}\right]$ & 12 & 12.03 & $(1.41)$ & 12.12 & ( 1.36$)$ \\
\hline $\mathbb{E}\left[\eta_{21} \mid \widehat{\Theta}\right]$ & 12 & 12.10 & ( 1.38$)$ & 12.05 & $(1.32)$ \\
\hline $\mathbb{E}\left[\eta_{22} \mid \widehat{\Theta}\right]$ & 19 & 19.03 & $(1.75)$ & 18.86 & $(1.70)$ \\
\hline$L R M_{1}$ & 2.632 & 2.652 & $(0.172)$ & 2.654 & $(0.184)$ \\
\hline$L R M_{2}$ & 2.632 & 2.656 & $(0.175)$ & 2.648 & $(0.175)$ \\
\hline runtime & & 75.79 & $(61.95)$ & 369.75 & $(329.47)$ \\
\hline
\end{tabular}

Table 5.11: Mean and standard deviations of estimators (250 simulations) when $n=p=$ 2 and using Algorithm II. Total number of free parameters is 13. 


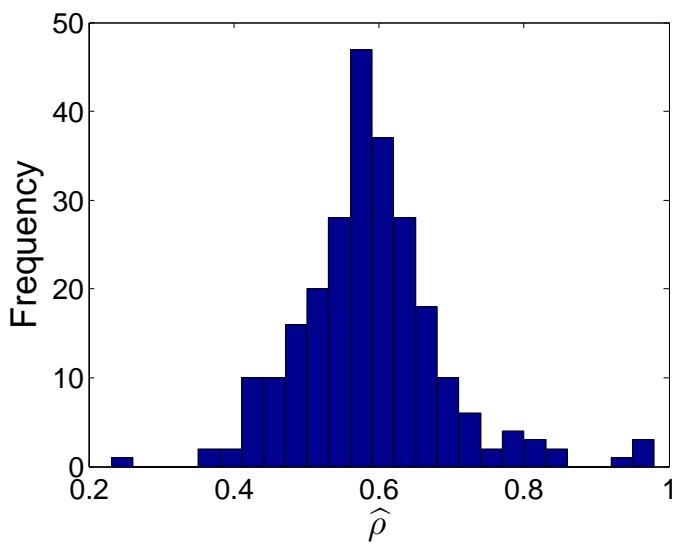

(a) Frequency of $\widehat{\rho}$

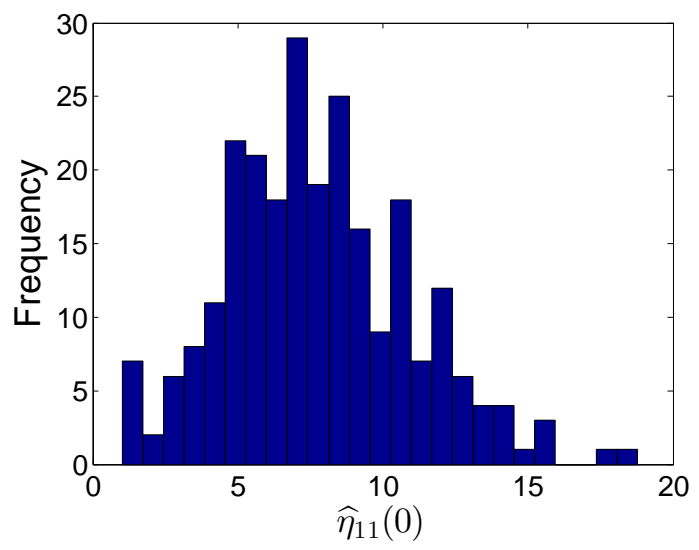

(c) Frequency of $\widehat{\eta}_{11}(0)$

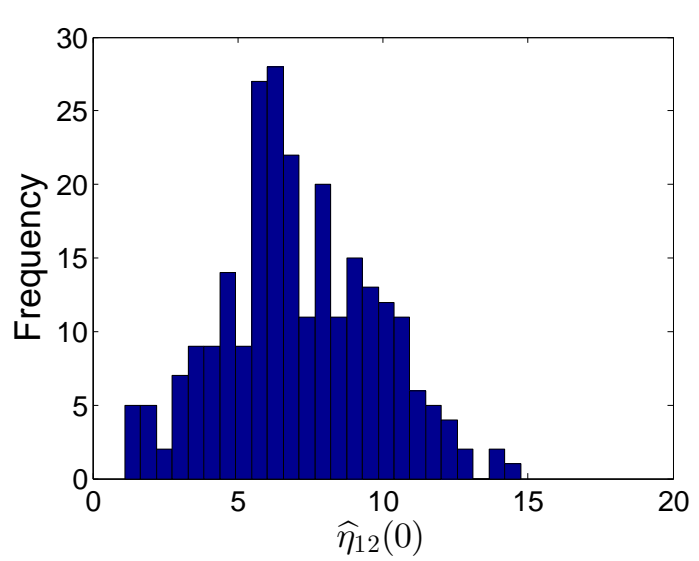

(e) Frequency of $\widehat{\eta}_{12}(0)$

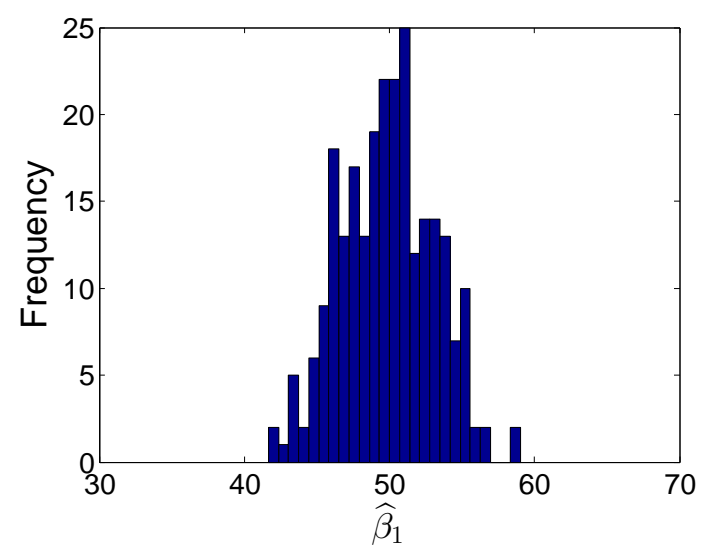

(b) Frequency of $\widehat{\beta}_{1}$

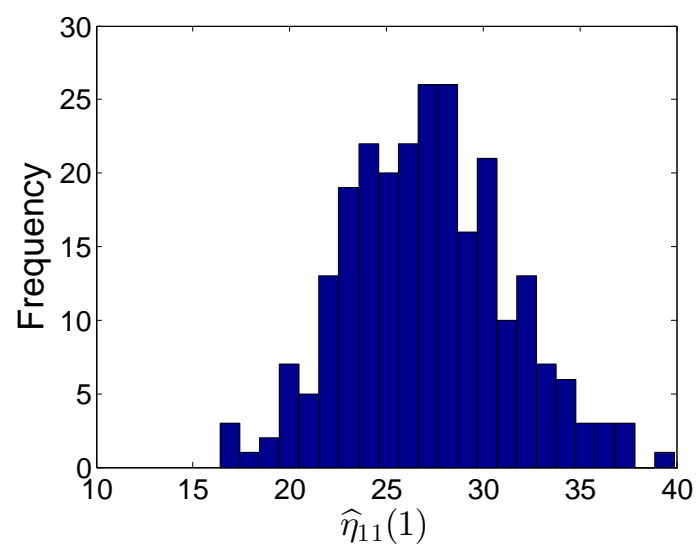

(d) Frequency of $\widehat{\eta}_{11}(1)$

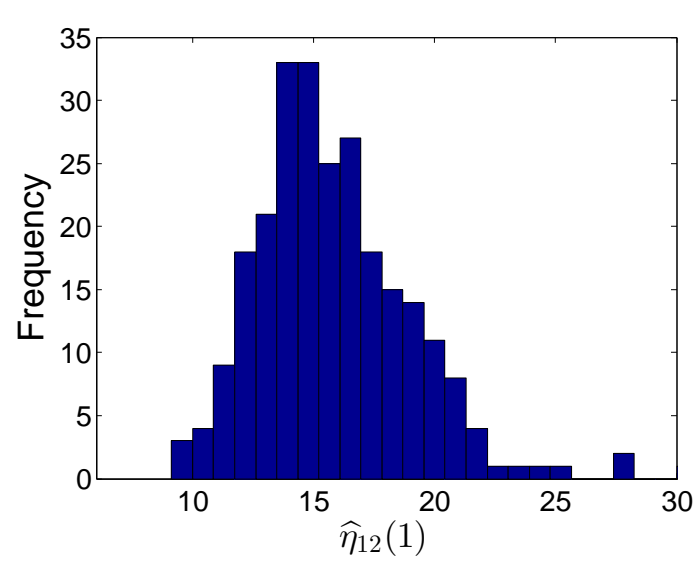

(f) Frequency of $\widehat{\eta}_{11}(2)$

Figure 5.8: Distribution of estimators (250 simulations of 2,000 data points) when $n=$ $p=2$ and using Algorithm II with 50 particles. This is the non-symmetric case. 


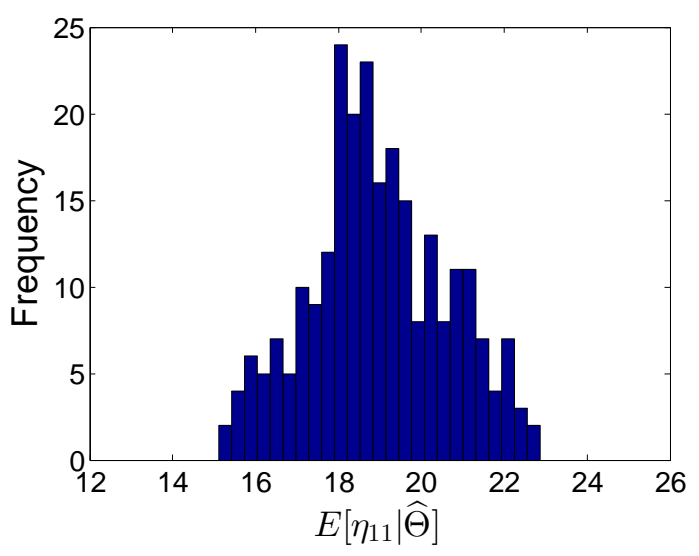

(a) Frequency of $\mathbb{E}\left(\eta_{11} \mid \widehat{\Theta}\right)$

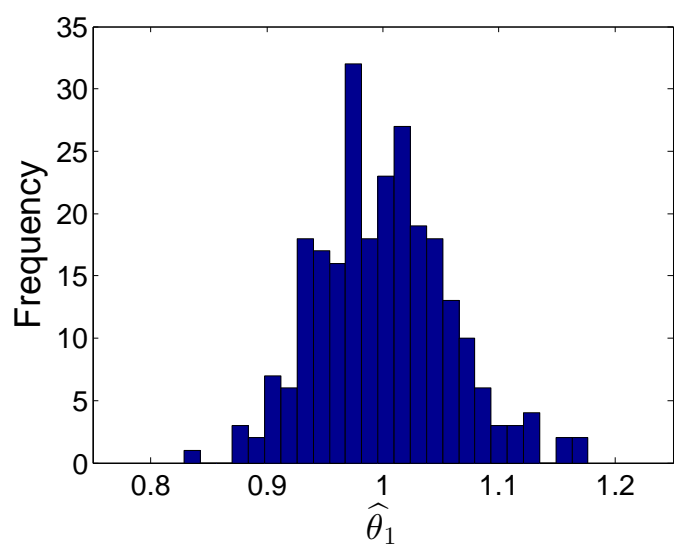

(c) Frequency of $\widehat{\theta}_{1}$

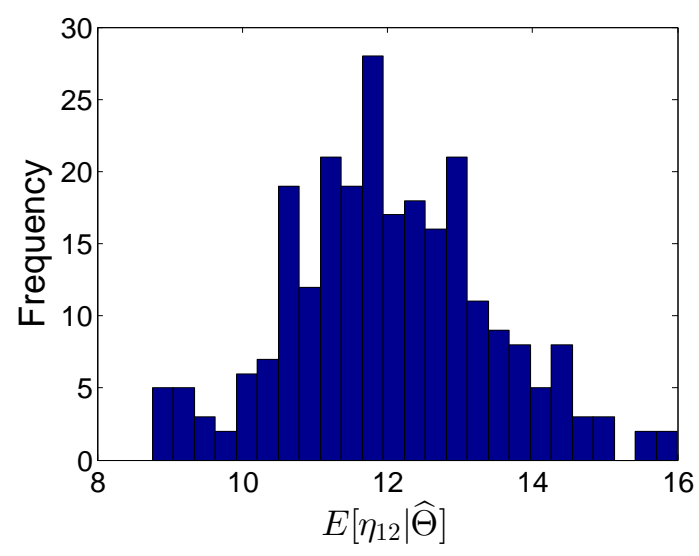

(b) Frequency of $\mathbb{E}\left(\eta_{12} \mid \widehat{\Theta}\right)$

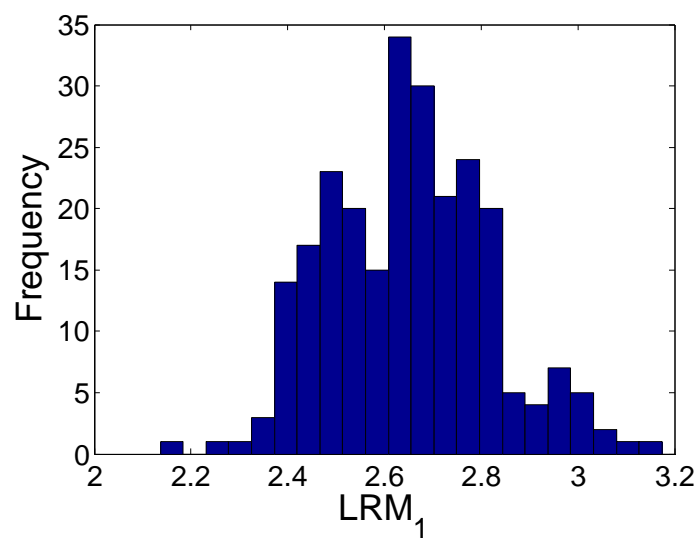

(d) Frequency of long run mean

Figure 5.9: Distribution of estimators (250 simulations of 2,000 data points) when $n=$ $p=2$ and using Algorithm II with 50 particles. This is the non-symmetric case. 


\section{$5.5 \overline{\mathcal{F}}_{t}$ - Adapted Estimators for $\boldsymbol{\lambda}_{t}$}

Sections 5.3 and 5.4 are useful in generating pseudo-likelihood estimators of $\Theta$. However, even with knowledge of the true value of $\Theta$, it is still no trivial task to recover the latent process $\boldsymbol{\lambda}_{t}$. This section provides two methods for real-time estimation of $\boldsymbol{\lambda}_{t}$.

\subsubsection{Via Renewal Points}

In this section, we propose a method to estimate the current value of $\boldsymbol{\lambda}_{t}$ after the calibration has been performed. Motivated by the subsequence selection criteria explained in Step 4 of Section 5.3.2, we compute an estimator for $\boldsymbol{\lambda}_{t}$ as follows: Suppose you want to compute $\hat{\boldsymbol{\lambda}}_{t}$ with $t \in\left[t_{k}, t_{k+1}\right)$.

1. Find $k_{0} \leq k$ such that $t_{k_{0}}$ is a renewal point, but $t_{k_{0}+1}, \ldots, t_{k}$ are not renewal points.

2. Assume $\boldsymbol{\lambda}_{t_{k_{0}}-}=\boldsymbol{\theta}$. Compute the $p^{k-k_{0}+1}$ possible values of $\boldsymbol{\lambda}_{t_{k}}$ (and hence, $\boldsymbol{\lambda}_{t}$ as well). Denote the possible values of $\boldsymbol{\lambda}_{t}$ as $\boldsymbol{\lambda}^{(i)}$.

3. Compute the posterior probability of each value of $\boldsymbol{\lambda}^{(i)}$ in Step 2 using the approximation

$$
\mathbb{P}\left[\boldsymbol{\lambda}_{t}=\boldsymbol{\lambda}^{(i)} \mid \overline{\mathcal{F}}_{t}\right] \approx \mathbb{P}\left[\boldsymbol{\lambda}_{t}=\boldsymbol{\lambda}^{(i)} \mid \sigma\left(\left\{\mathbf{N}_{u}\right\}_{u \in\left[t_{k_{0}}, t\right]}\right), \boldsymbol{\lambda}_{t_{k_{0}}}=\boldsymbol{\theta}\right]
$$

The details of this computation can be found in Appendix E.4.

4. Take the estimator $\hat{\boldsymbol{\lambda}}_{t}$ to be the posterior mean. More generally, one could take $\hat{\boldsymbol{\lambda}}_{t}$ such that $h\left(\hat{\boldsymbol{\lambda}}_{t}\right)$ is (conditional on the observed data) unbiased for $h\left(\boldsymbol{\lambda}_{t}\right)$, where $h: \mathbb{R}_{+}^{n} \rightarrow \mathbb{R}^{n}$.

Observe that the simplifying approximation in Step 2 becomes more accurate as $\epsilon \downarrow 0$. However, the smaller $\epsilon$ is, the more inefficient this algorithm becomes since (for a fixed $t$ ) $k_{0}$ decreases as $\epsilon$ decreases. Hence, the number of total paths can quickly become quite large if $\epsilon$ is unreasonably small.

\subsubsection{Via Sequential Monte Carlo}

This method was used in producing this chapter's motivating plots in Figure 5.1. Furthermore, we propose this to be the method used in constructing on-the-fly estimators 
for $\boldsymbol{\lambda}_{t}$ for use in the optimal HFT strategy in the setting of Chapter 3.

Section 5.4.1 outlines the classical method for particle filtering using Sequential Monte Carlo. However, we need a way to estimate $\boldsymbol{\lambda}_{t_{k}}$ that is $\overline{\mathcal{F}}_{t_{k}}$ measurable. Unfortunately, classical SMC methods do not address this issue, nor do they provide a way to estimate $\boldsymbol{\lambda}_{t}$ when $t$ is not the arrival time of an event. That is, we seek an estimator $\hat{\boldsymbol{\lambda}}_{t}$ such that: If $\mathcal{G}$ is a $\sigma$-algebra on $\Omega$ with $\hat{\boldsymbol{\lambda}}_{t} \in \mathcal{G}$, then for any event time $t_{k} \leq t$, we must have $\mathcal{G} \subseteq \overline{\mathcal{F}}_{t_{k}}$ if and only if $t=t_{k}$.

To this end, we first consider the state of the particles at $t_{k-1}$ via classical SMC methods (as in Section 5.4.1). We can then use the survival probability (no event yet after the $k^{\text {th }}$ event) to find the posterior probability that the $k^{\text {th }}$ event induced a particular jump $\boldsymbol{\eta}_{k}$, propagate the particles forward to get an estimate of the posterior distribution of $\boldsymbol{\lambda}_{t_{k}}$, and exponentially decay this value forward to find $\boldsymbol{\lambda}_{t}$. The full details of this modified SMC particle filter are given as follows:

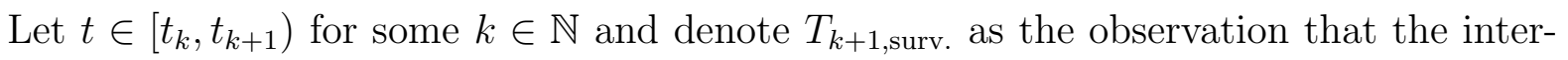
arrival time of the $(k+1)^{\text {th }}$ event is right truncated at $t-t_{k}$.

1. Using the method in Section 5.4.1, propagate the particles to $t_{k-1}$.

2. Draw $M$ samples of $\boldsymbol{\lambda}_{t_{k}}$ from the posterior distribution

$$
\boldsymbol{\lambda}_{t_{k}}^{(i)} \sim \mathbb{P}\left[\boldsymbol{\lambda}_{t_{k}} \mid \boldsymbol{\lambda}_{t_{k-1}}, T_{k}, T_{k+1, \text { surv. }}\right] .
$$

(a) Compute $l_{k, \text { surv. }}(q):=l_{\text {surv. }}\left(q \mid \boldsymbol{\lambda}_{t_{k-1}}, T_{k}, T_{k+1, \text { surv. }}\right)$ (i.e. probability that $H_{n-1}=$ $q$, for $q=1, \ldots, p-1$, given $\boldsymbol{\lambda}_{t_{k-1}}, T_{k}$, and $\left.T_{k+1, \text { surv. }}\right)$ where $l_{\text {surv. }}\left(q \mid y, S_{1}, S_{2}\right)$ is provided in Observation 5.2.4.

(b) Draw iid uniforms $u^{(i)} \sim U(0,1)$ for each $i=1, \ldots, M$

(c) Use the uniform $u^{(i)}$ to simulate and set $H_{k}^{(i)}=q \in\{0,1, \ldots, p-1\}$ with corresponding probability $l_{k, \text { surv. }}(q)$. That is, set

$$
H_{k-1}^{(i)}=\sup \left\{q \in\{0,1, \ldots, p-1\}: \sum_{z=0}^{q-1} l_{k, \text { surv. }}(z) \leq u^{(i)}\right\}
$$

(d) Set

$$
\boldsymbol{\lambda}_{t_{k}}^{(i)}=\boldsymbol{\theta}+e^{-\boldsymbol{\beta} T_{k}(1)}\left(\boldsymbol{\lambda}_{t_{k-1}}^{(i)}-\boldsymbol{\theta}\right)+\boldsymbol{\eta}\left(H_{k}^{(i)}\right) e_{T_{k}(2)}
$$


3. Compute the un-normalized weights, given the observation $T_{k+1 \text {,surv. }}$

$$
\begin{aligned}
\alpha^{(i)} & =\frac{\mathbb{P}\left[T_{k+1, \text { surv. }} \mid \boldsymbol{\lambda}_{t_{k}}=\boldsymbol{\lambda}_{t_{k}}^{(i)}\right] \mathbb{P}\left[H_{k}=H_{k}^{(i)} \mid \boldsymbol{\lambda}_{t_{k-1}}=\boldsymbol{\lambda}_{t_{k-1}}^{(i)}, T_{k}\right]}{\mathbb{P}\left[H_{k}=H_{k}^{(i)} \mid \boldsymbol{\lambda}_{t_{k-1}}=\boldsymbol{\lambda}_{t_{k-1}}^{(i)}, T_{k}, T_{k+1, \text { surv. }}\right]} \\
& =g_{\text {surv. }}\left(T_{k+1, \text { surv. }} \mid \boldsymbol{\lambda}_{t_{k}}^{(i)}\right) \times \frac{\rho_{H_{k}^{(i)}}}{l_{k, \text { surv. }}\left(H_{k}^{(i)}\right)}
\end{aligned}
$$

where $g(t, l \mid y)$ is provided by Equation (5.7) in Observation 5.2.3.

4. Compute and resample from the normalized weights.

(a) $W^{(i)}=\alpha^{(i)} / \sum_{j} \alpha^{(j)}$

(b) Draw a single uniform $u \sim U(0,1)$ and define $u_{i}=\frac{u+i-1}{M}, i=1, \ldots, M$

(c) Find $N_{i}$ such that $\sum_{j=1}^{N_{i}-1} W^{(j)} \leq u_{i} \leq \sum_{j=1}^{N_{i}} W^{(j)}$. The number of "offspring" for particle $i$ is given by $M_{i}=N_{i}-N_{i-1}$ (with $\left.N_{0}=0\right)$

(d) For each $\boldsymbol{\lambda}_{t_{k}}^{(i)}$, create $M_{i}$ offspring and call these $\overline{\boldsymbol{\lambda}}_{t_{k}}^{(i)}$

(e) The updated equally weighted paths of the particles are $\overline{\boldsymbol{\lambda}}_{t_{1}}^{(i)}, \ldots, \overline{\boldsymbol{\lambda}}_{t_{k-1}}^{(i)}$

5. Decay the resulting particles at $t_{k}$ exponentially with decay rate matrix $\boldsymbol{\beta}$ to get

$$
\overline{\boldsymbol{\lambda}}_{t}^{(i)}=\boldsymbol{\theta}+e^{-\boldsymbol{\beta}\left(t-t_{k}\right)}\left(\overline{\boldsymbol{\lambda}}_{t_{k}}^{(i)}-\boldsymbol{\theta}\right) .
$$

Note that we have already resampled under the posterior probabilities given this survival event. Hence, the particles at time $t$ still have uniform probability.

See Figure 5.10 for a visual depiction of the mean of 3 particles propagating through time. At any time $t$, the value of the particle is conditional on $\overline{\mathcal{F}}_{t}$, not $\overline{\mathcal{F}}_{t_{k}}$. Observe how in some instances the particles experience sudden drops during exponential decay. This is due to the fact that just after the arrival of an event, there is little information about whether or not the event induced a jump in the particle. However, as time evolves with the absence of the arrival of the next event, the posterior probability that the previous event induced a large jump decreases and eventually some particles now do not experience the aforementioned jump. Hence, there is a downwards jump in the adapted propagation of such particles. This is easy to visualize due to the small number of particles used in the SMC particle filter (only 3 in this plot). As the number of particles becomes large, this effect will be smoothed out and will cause the mean of all the particles to decay faster than rate $\boldsymbol{\beta}$ (the decay may no longer be exponential, but the resulting intensity is less than that if it decayed exponentially with rate $\boldsymbol{\beta}$ ). 


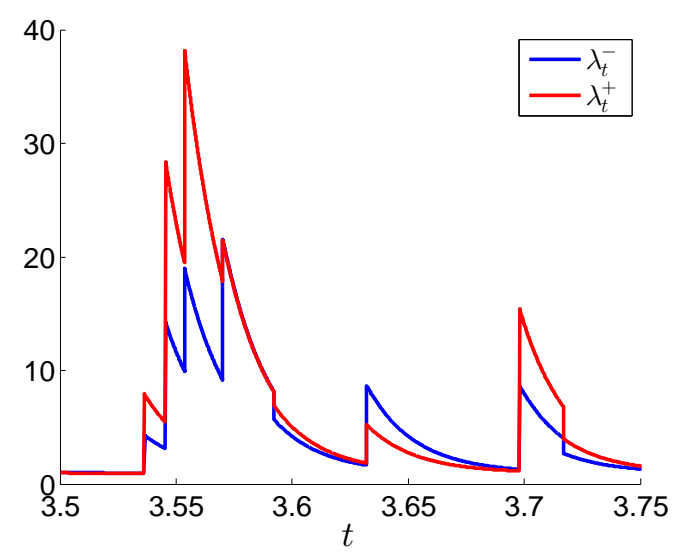

Figure 5.10: Propagation of the progressively measureable particle filter using the mean of 3 particles via SMC with simulated data.

Furthermore, the mean jump size in the particles at the next event will be at least $\mathbb{E}[\boldsymbol{\eta}]$. If the next event occurs shortly after the previous one, then some of the particles may exhibit an additional jump due to the fact that the previous event may now be considered to be one that induces a large jump in $\boldsymbol{\lambda}_{t}$. Such behaviour is a byproduct of the posterior probability of the previous point causing a large jump in $\boldsymbol{\lambda}_{t}$ being high when the next event occurs very shortly thereafter. Because the particle propagation must be $\overline{\mathcal{F}}_{t^{-}}$adapted, this posterior probability does not increase until the time of the next event.

To get the point estimator $\hat{\boldsymbol{\lambda}}_{t}$, first notice that conditional on $\overline{\mathcal{F}}_{t}$, the posterior probability of each particle is equal. With a slight abuse of notation, denote the $i^{\text {th }}$ particle as $\boldsymbol{\lambda}_{t}^{(i)}$. Observe that the estimators $\hat{\boldsymbol{\lambda}}_{t}=\frac{1}{M} \sum_{i=1}^{M} \boldsymbol{\lambda}_{t}^{(i)}$ are approximately unbiased for $\boldsymbol{\lambda}_{t}$ (given the calibrated parameters are correct), where $M$ is the number of particles. The approximation is due to the left limit of $\boldsymbol{\lambda}$ at renewal points being very close to $\boldsymbol{\theta}$, but not actually equal to $\boldsymbol{\theta}$. Since the conditional distribution of $\boldsymbol{\lambda}_{t}$ is uniform over the particles, computing unbiased estimators for a given function of $\boldsymbol{\lambda}_{t}$ is a trivial numerical task.

\subsection{Other Applications}

Within the context of quantitative finance, self-exciting latent processes can be used to model the default times of corporate bonds in multiple sectors. This has been studied in Azizpour and Giesecke (2008), where the classical Hawkes process is applied to such data. The authors also provide filtering methods when the intensity process is masked by a latent diffusion process. The extension to the case where the jump size for each event 
is both latent and random is a natural one as the default of a large cap corporate bond would have a very different effect on a macroscopic level than that of a start-up firm.

The use of the multivariate Hakwes model in market microstructure has been studied in a variety of flavours. Large (2007) explores a stylized framework where he distinguishes between market orders (MOs) and limit orders (LOs) based on whether or not they cause a change in the market price. Bacry and Muzy (2013) jointly model the arrival of MOs and the up/down ticks in a 4-dimensional Hawkes process.

Continuing with this theme, one could attempt to (exogenously) model just the tick movements in a way that also distinguishes between an uptick due to the bid price increasing versus the ask price increasing for the purposes of price and liquidity prediction. These two events are fundamentally different as the former results in a tighter bid/offer spread, whereas the latter results in a wider spread. This subtle distinction is typically lost in the modelling choice (although it is recoverable in the framework that Large (2007) poses).

To this end, one could classify market movements as one of four types: the best bid price moves up or down, and the best offer price moves up or down. The cause of such movements can be one of three basic types (MO submission, LO cancellation, LO submitted inside the spread). It is observed that certain movements in the BBO tend to cluster together and cross-excite other movements of a particular type. For example, an increase in the best offer price may be likely to induce the arrival of LOs to the offer side causing the next event to be a reduction in the best offer price. Or perhaps the data suggests that an increase in the best offer will self-excite more increases of the same type, but will also cross-excite increases in the best bid price. This will ultimately create a short term upward pressure on the price for this asset.

Finally, as eluded to at the beginning of this chapter, there are several cross-disciplinary applications of the Classical Hawkes Model that the author feels are also worth exploring in the context of the more generalized process presented in Section 5.2.1. 


\section{Chapter 6}

\section{Application to Real Data}

This chapter applies some of the theoretical results developed throughout previous chapters of this thesis to real tick-by-tick event data for two equities trading on the NASDAQ exchange and one equity on the NYSE. Sections 6.1 and 6.2 apply the calibration methodologies of Sections 5.3 (Algorithm I) and 5.4 (Algorithm II), respectively, where parameter estimates and some model adequacy diagnostics are reported. Section 6.3 discusses the performance of the single asset market making strategy derived in Chapter 3 (with a slight modification for market orders, described in Section 6.3.1). Parameter estimates from Section 6.1.1, combined with the modified on-the-fly particle filtering method developed in Section 5.5.2, are the tools used to construct on-the-fly estimates of $\boldsymbol{\lambda}_{t}$ (adapted to the smaller, observable filtration $\overline{\mathcal{F}}_{t}$ ).

\subsection{Statistical Results: Generalized Latent Hawkes Process when $\boldsymbol{\eta}(0)=0$}

In this section, we apply Algorithm I with cluster size $m=4$, as described in Section 5.3.2, to the symmetric 2-dimensional Hawkes model used in Chapter 3, as well as the non-symmetric case. For this entire section, we impose the constraint that $\boldsymbol{\eta}(0)=\mathbf{0}$.

\subsubsection{Parameter Estimates}

The raw data is comprised of LOB events which classifies all events as executed market orders (MOs), limit order (LO) submissions, and LO cancellations. A typical trading day 


\begin{tabular}{crrrrrrrr}
\hline \hline & \multicolumn{2}{c}{ BBBY } & & \multicolumn{2}{c}{ MSFT } & & \multicolumn{2}{c}{ TEVA } \\
\cline { 2 - 3 } \cline { 8 - 9 } & mean & $($ std $)$ & & mean & $($ std $)$ & & mean & (std) \\
\hline$\widehat{\rho}$ & 0.776 & $(0.171)$ & & 0.412 & $(0.087)$ & & 0.766 & $(0.171)$ \\
$\widehat{\beta}$ & 97.42 & $(27.13)$ & & 105.35 & $(27.13)$ & & 115.64 & $(33.01)$ \\
$\widehat{\eta}_{11}(1)$ & 49.16 & $(16.05)$ & & 125.76 & $(31.53)$ & & 61.57 & $(18.59)$ \\
$\widehat{\eta}_{12}(1)$ & 6.57 & $(2.37)$ & & 19.39 & $(6.62)$ & & 11.88 & $(4.16)$ \\
$\widehat{\theta}$ & 0.085 & $(0.041)$ & & 0.157 & $(0.075)$ & & 0.105 & $(0.054)$ \\
\hline $\mathbb{E}\left[\eta_{11} \mid \widehat{\Theta}\right]$ & 36.80 & $(10.87)$ & & 51.53 & $(15.75)$ & & 45.89 & $(13.46)$ \\
$\mathbb{E}\left[\eta_{12} \mid \widehat{\Theta}\right]$ & 4.95 & $(1.72)$ & & 7.92 & $(2.89)$ & & 8.97 & $(3.44)$ \\
$L R M$ & 0.151 & $(0.076)$ & & 0.371 & $(0.240)$ & & 0.206 & $(0.117)$ \\
\hline \hline
\end{tabular}

Table 6.1: Mean and standard deviations of estimators on historical data when $n=p=2$, $\boldsymbol{\eta}(0)=\mathbf{0}, \eta_{11}(1)=\eta_{22}(1), \eta_{12}(1)=\eta_{21}(1), \beta_{1}=\beta_{2}, \theta_{1}=\theta_{2}$, and using Algorithm I with a cluster size of $m=4$. Total number of free parameters is 5. $L R M$ refers to the long run mean of activity rate of any component of $\mathbf{N}_{t}$, given by Lemma 3.2.2. Sample consists of disjoint 60-minute windows between 10:00am and 4:00pm from Jan-May 2011.

could have anywhere from 200,000 to 1,000,000 total events per equity, of which contain 5,000 to 50,000 market orders (after amalgamation - the exchange records a separate entry when a MO fills multiple LO quotes; partial fills are also treated as a single MO). The LOB must be reconstucted from this raw data by keeping track of all existing LO quotes and updating it with submissions, cancellations, and executions.

We perform a calibration to disjoint 60 minute windows between 10:00am and 4:00pm, for the months January to May of 2011. Although each window is treated equally in the sample, this could be refined by considering different periods throughout the trading day (morning, afternoon, and close). The analysis is performed for the three tickers BBBY (Bed Bath and Beyond Inc.), MSFT (Microsoft Corporation), and TEVA (Teva Pharmaceutical Industries Ltd). See Tables 6.1 and 6.2 for results for the symmetric model (as described in Chapter 3) and its non-symmetric version, respectively. We also provide histograms of the fitted parameters for the symmetric model in Figure 6.1.

Observe from Table 6.2 that even if we do not impose the symmetry conditions in the calibration, that the maximum likelihood estimators (MLEs) still produce symmetric estimates of the parameters (in mean). A simple check to see if there is negative correlation between these parameters (they can still be equal in mean, but be heavily negatively correlated) is to check their empirical correlation. Table 6.3 shows that the empirical correlations for all the quantities in question are positive. In favour of sparsity, we continue the analysis in this section only focusing on the symmetric model. 


\begin{tabular}{|c|c|c|c|c|c|c|}
\hline & \multicolumn{2}{|c|}{ BBBY } & \multicolumn{2}{|c|}{ MSFT } & \multicolumn{2}{|c|}{ TEVA } \\
\hline & mean & $(\mathrm{std})$ & mean & $(\mathrm{std})$ & mean & $(\mathrm{std})$ \\
\hline$\widehat{\rho}$ & 0.789 & $(0.168)$ & 0.419 & $(0.088)$ & 0.777 & $(0.168)$ \\
\hline$\widehat{\beta}_{1}$ & 99.43 & $(36.79)$ & 107.07 & $(33.61)$ & 119.01 & $(44.02)$ \\
\hline$\widehat{\beta}_{2}$ & 100.60 & $(37.12)$ & 107.74 & $(32.74)$ & 117.37 & $(38.16)$ \\
\hline$\widehat{\eta}_{11}(1)$ & 49.95 & $(20.49)$ & 124.87 & $(38.22)$ & 63.32 & $(23.13)$ \\
\hline$\widehat{\eta}_{12}(1)$ & 6.91 & $(3.14)$ & 20.85 & $(8.73)$ & 12.47 & $(5.58)$ \\
\hline$\widehat{\eta}_{21}(1)$ & 6.35 & $(3.26)$ & 19.23 & $(8.69)$ & 11.74 & $(5.53)$ \\
\hline$\widehat{\eta}_{22}(1)$ & 49.61 & $(20.95)$ & 129.48 & $(39.37)$ & 60.63 & $(22.37)$ \\
\hline$\widehat{\theta_{1}}$ & 0.085 & $(0.042)$ & 0.163 & $(0.084)$ & 0.104 & $(0.056)$ \\
\hline$\widehat{\theta}_{2}$ & 0.084 & $(0.044)$ & 0.149 & $(0.082)$ & 0.105 & $(0.057)$ \\
\hline $\mathbb{E}\left[\eta_{11} \mid \widehat{\Theta}\right]$ & 38.16 & $(14.57)$ & 51.99 & $(18.03)$ & 48.05 & $(17.43)$ \\
\hline $\mathbb{E}\left[\eta_{12} \mid \widehat{\Theta}\right]$ & 5.31 & $(2.40)$ & 8.59 & $(3.60)$ & 9.57 & $(4.65)$ \\
\hline $\mathbb{E}\left[\eta_{21} \mid \widehat{\Theta}\right]$ & 4.85 & $(2.31)$ & 7.95 & $(3.57)$ & 8.93 & $(4.15)$ \\
\hline $\mathbb{E}\left[\eta_{22} \mid \widehat{\Theta}\right]$ & 37.72 & $(14.43)$ & 53.49 & $(17.50)$ & 45.70 & $(15.77)$ \\
\hline$L R M_{1}$ & 0.155 & $(0.079)$ & 0.389 & $(0.257)$ & 0.209 & $(0.123)$ \\
\hline$L R M_{2}$ & 0.150 & $(0.078)$ & 0.364 & $(0.244)$ & 0.204 & $(0.117)$ \\
\hline
\end{tabular}

Table 6.2: Mean and standard deviations of estimators on historical data when $n=p=2$, $\boldsymbol{\eta}(0)=\mathbf{0}$, and using Algorithm I with a cluster size of $m=4$. $L R M$ refers to the long run mean of activity rate of $\mathbf{N}_{t}$, given by Lemma 3.2.2. Sample consists of disjoint 60-minute windows between 10:00am and 4:00pm from Jan-May 2011.

\begin{tabular}{cccc}
\hline \hline & BBBY & MSFT & TEVA \\
\hline $\operatorname{corr}\left(\widehat{\beta}_{1}, \widehat{\beta}_{2}\right)$ & 0.074 & 0.208 & 0.267 \\
$\operatorname{corr}\left(\widehat{\eta}_{11}(1), \widehat{\eta}_{22}(1)\right)$ & 0.220 & 0.206 & 0.294 \\
$\operatorname{corr}\left(\widehat{\eta}_{12}(1), \widehat{\eta}_{21}(1)\right)$ & 0.186 & 0.234 & 0.176 \\
$\operatorname{corr}\left(\widehat{\theta}_{1}, \widehat{\theta}_{2}\right)$ & 0.812 & 0.618 & 0.828 \\
\hline \hline
\end{tabular}

Table 6.3: Sample correlation of selected pairs of estimators when the calibration does not impose symmetry. 


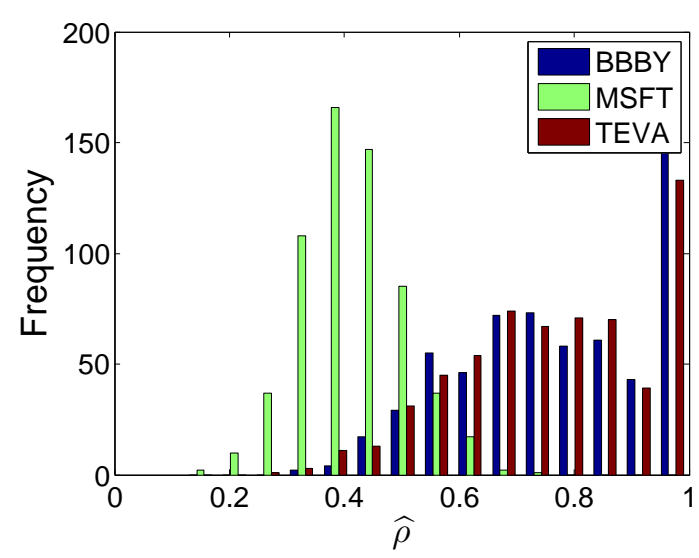

(a) Frequency of $\widehat{\rho}$

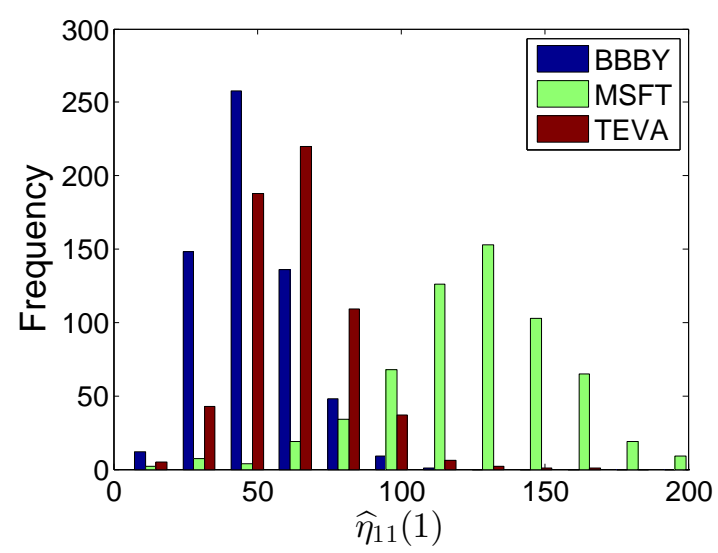

(c) Frequency of $\widehat{\eta}_{11}(1)$

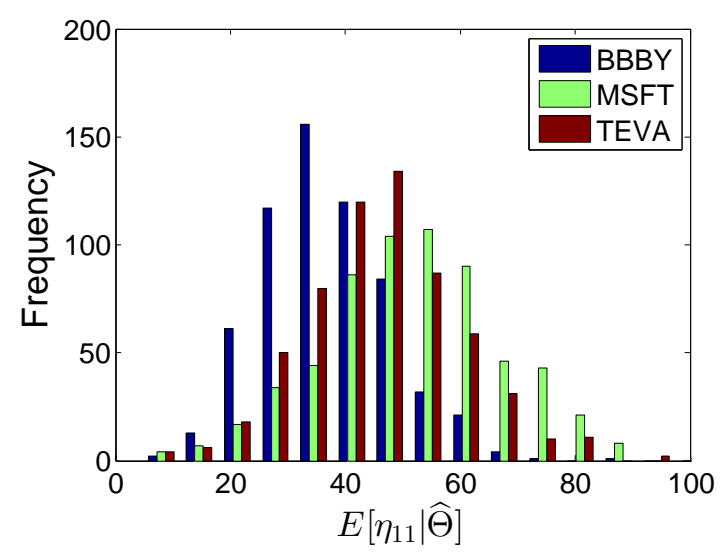

(e) Frequency of $\mathbb{E}\left(\eta_{11} \mid \widehat{\Theta}\right)$

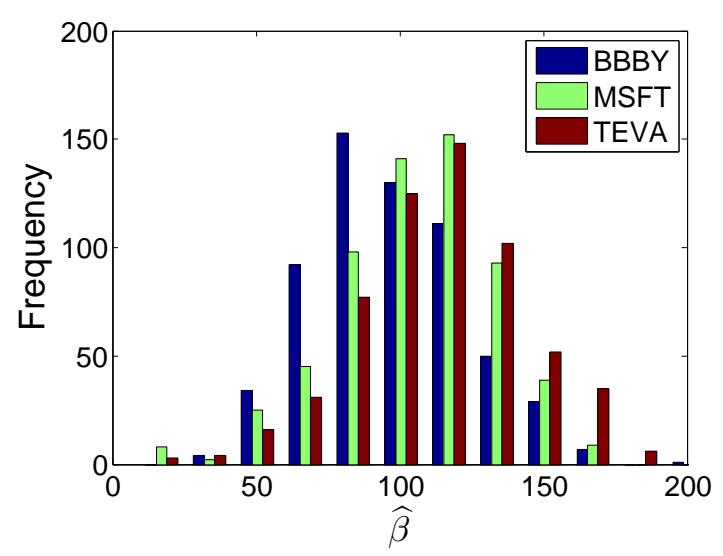

(b) Frequency of $\widehat{\beta}$

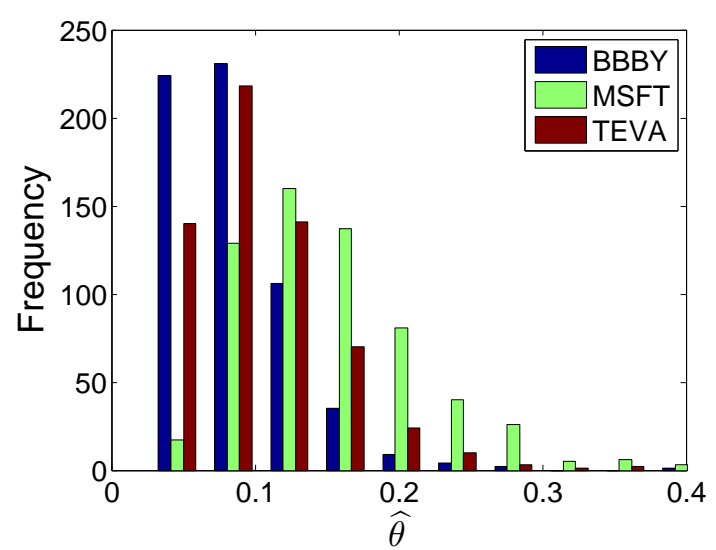

(d) Frequency of $\widehat{\theta}$

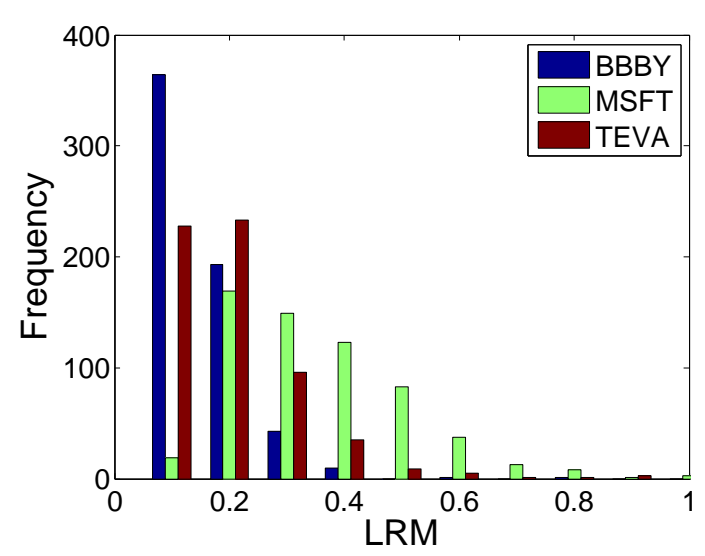

(f) Frequency of LRM

Figure 6.1: Frequency of parameter estimates when constrained to $n=p=2, \boldsymbol{\eta}(0)=\mathbf{0}$, $\eta_{11}(1)=\eta_{22}(1), \eta_{12}(1)=\eta_{21}(1), \beta_{1}=\beta_{2}, \theta_{1}=\theta_{2}$, and using Algorithm I. Sample consists of disjoint 60-minute windows between 10:00am and 4:00pm from Jan-May 2011. 


\begin{tabular}{crrr}
\hline \hline & BBBY & \multicolumn{1}{c}{ MSFT } & \multicolumn{1}{c}{ TEVA } \\
\hline$N$ & 546 & 1347 & 1286 \\
\hline$\widehat{\rho}$ & 0.701 & 0.331 & 0.564 \\
$\widehat{\beta}$ & 118.34 & 66.94 & 103.37 \\
$\widehat{\eta}_{11}(1)$ & 69.17 & 103.56 & 82.60 \\
$\widehat{\eta}_{12}(1)$ & 10.60 & 10.24 & 17.39 \\
$\widehat{\theta}$ & 0.041 & 0.084 & 0.082 \\
\hline \hline
\end{tabular}

Table 6.4: Parameter estimates used for PIT and P-P model diagnotics for the 2:00-3:00 window of May 31, 2011.

\begin{tabular}{crrr}
\hline \hline & BBBY & MSFT & \multicolumn{1}{c}{ TEVA } \\
\hline$N$ & 1526 & 2898 & 1738 \\
\hline$\widehat{\rho}$ & 0.482 & 0.373 & 0.605 \\
$\widehat{\beta}$ & 90.93 & 80.02 & 100.63 \\
$\widehat{\eta}_{11}(1)$ & 69.54 & 91.83 & 59.72 \\
$\widehat{\eta}_{12}(1)$ & 8.42 & 14.66 & 11.63 \\
$\widehat{\theta}$ & 0.126 & 0.176 & 0.137 \\
\hline \hline
\end{tabular}

Table 6.5: Parameter estimates used for PIT and P-P model diagnotics for the 3:00-4:00 window of May 31, 2011.

\subsubsection{Model Validation: Probability Integral Transform}

All model diagnostics performed in this section are done with isolated likelihood cluster size $m$ equal to 4 for Algorithm I from Section 5.3.2. We use data from the last two 60-minute windows (2:00-3:00 and 3:00-4:00) of the last day in the period used in Section 6.1 (May 31, 2011). The parameter estimates for these 60-minute windows can be found in Tables 6.4 and 6.5, respectively.

The Probability Integral Transform (PIT; see Thorarinsdottir (2013)) is a transformation of each interarrival time into a random variable supported on the unit interval. Under the true model, this is an iid sequence of uniform random variables on $[0,1]$. Exact PIT quantites can be computed using the following decomposition:

$$
\begin{aligned}
& \mathbb{P}\left[T_{k}(1)>T_{k, \text { obs }}(1) \mid \overline{\mathcal{F}}_{t_{k-1}}\right]= \\
& \sum_{i} \mathbb{P}\left[T_{k}(1)>T_{k, \text { obs }}(1) \mid \boldsymbol{\lambda}_{t_{k-1}}=\boldsymbol{\lambda}^{(i)}\right] \mathbb{P}\left[\boldsymbol{\lambda}_{t_{k-1}}=\boldsymbol{\lambda}^{(i)} \mid \overline{\mathcal{F}}_{t_{k-1}}\right] .
\end{aligned}
$$




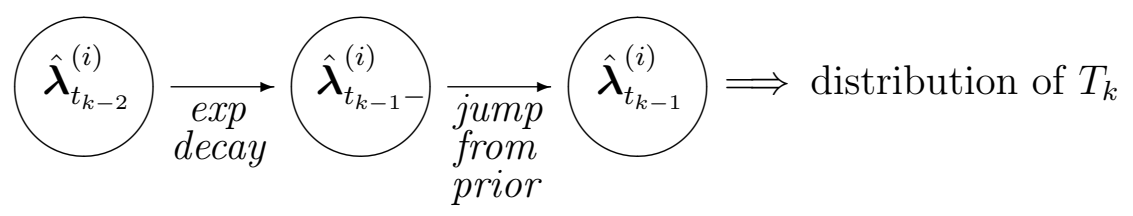

Figure 6.2: A workflow diagram representing the PIT calculation of the $i^{\text {th }}$ particle for the $k^{\text {th }}$ interarrival time $T_{k}$.

Further notice that the last probability is a posterior probability of a particular value of $\boldsymbol{\lambda}$ given the data. The details regarding the computation of this quantity can be found in Appendix E.4. Since we are conditioning on $\overline{\mathcal{F}}_{t_{k-1}}$ (the filtration at the time of an event), we do not need to include a survival probability since the last event.

Note that time to compute the posterior distribution of $\boldsymbol{\lambda}_{t}$ at the end of a cluster of size $n$ is $O\left(2^{n}\right)$. Due to this computational complexity, we use Sequential Monte Carlo (SMC) with 100 particles to efficiently approximate the PIT quantities. To calculate the survival probability associated with the $k^{\text {th }}$ interarrival time, we first propagate the particles to $t_{k-2}$, then decay them exponentially to $t_{k-1}$ and add a jump with probability $\rho$. A workflow diagram of this method is given in Figure 6.2. As discussed in Section 5.5 .2 , the state of the particle at $t_{k-1}$ must be $\overline{\mathcal{F}}_{t_{k-1}}$ measurable and, as described there, this induces some subtlety in its simulation.

Figures 6.3 and 6.4 show the distribution of the PIT quantities (for comparison purposes, the Poisson case is also shown). If the model is correct, then the PIT should produce iid uniform- $(0,1)$ random variables. A higher order test for uncorrelated uniforms is to look at the sample auto-correlation function. Figures 6.5 and 6.6 show the corresponding auto-correlation functions.

Observe the drastic improvement over the poisson case (the constant mean of the poisson case is taken to be equal to the long run mean in the full model). The autocorrelation at several lags is also statistically significantly positive when the poisson model is assumed, which is consistent with the notion of self-excitation of event arrivals. Although there are some lags for the Generalized Hawkes model that are statistically significantly different from zero, the deviation from zero autocorrelation and level of significance is far more prominent in the case of the Poisson process. Some deviation is expected since we acknowledge that the parsimonious model we are fitting is not $100 \%$ perfect. 


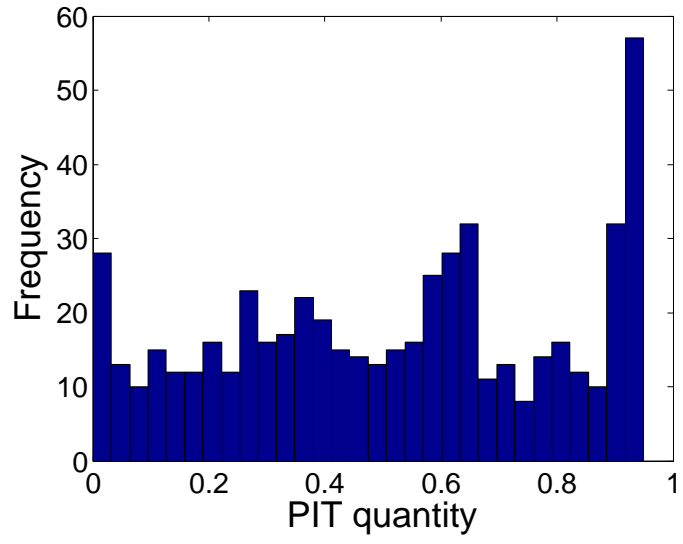

(a) Generalized Hawkes: BBBY

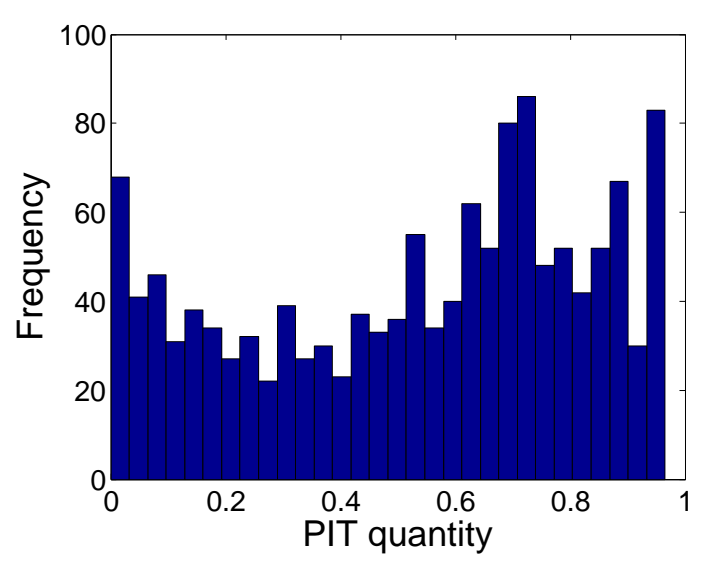

(c) Generalized Hawkes: MSFT

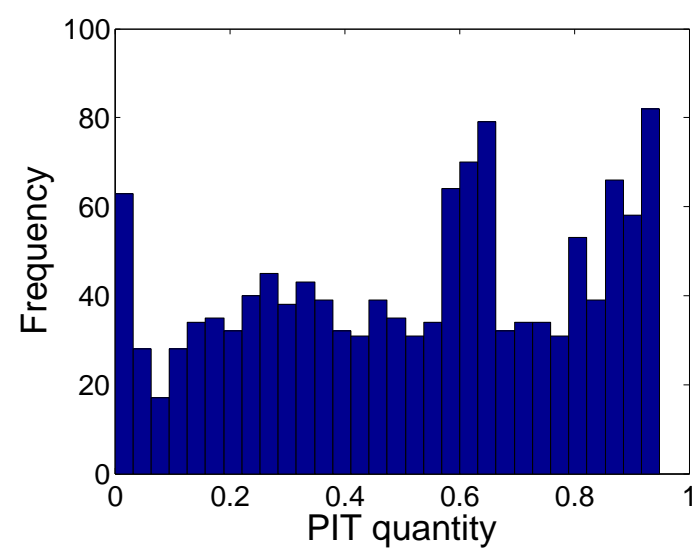

(e) Generalized Hawkes: TEVA

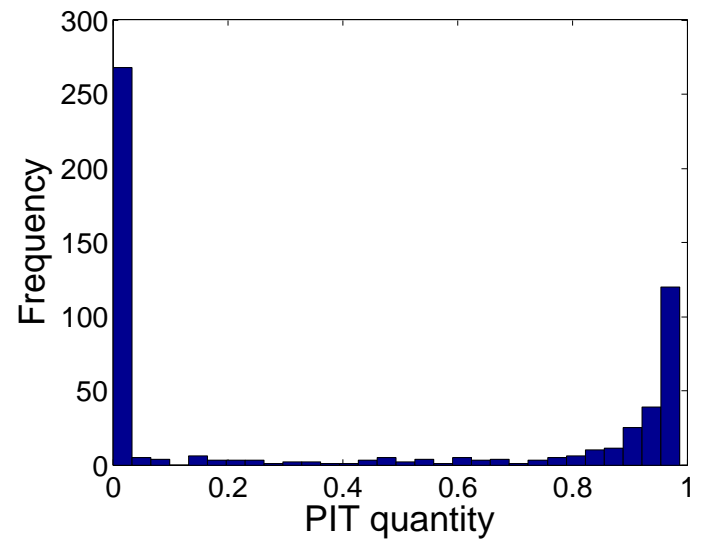

(b) Poisson: BBBY

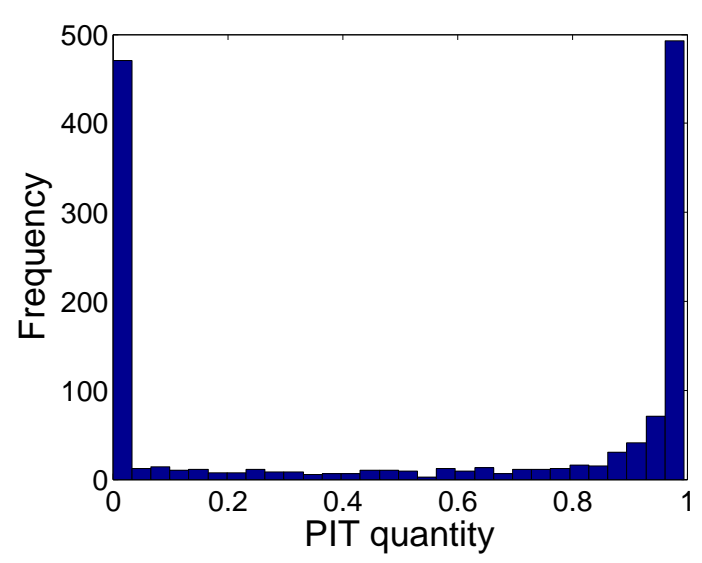

(d) Poisson: MSFT

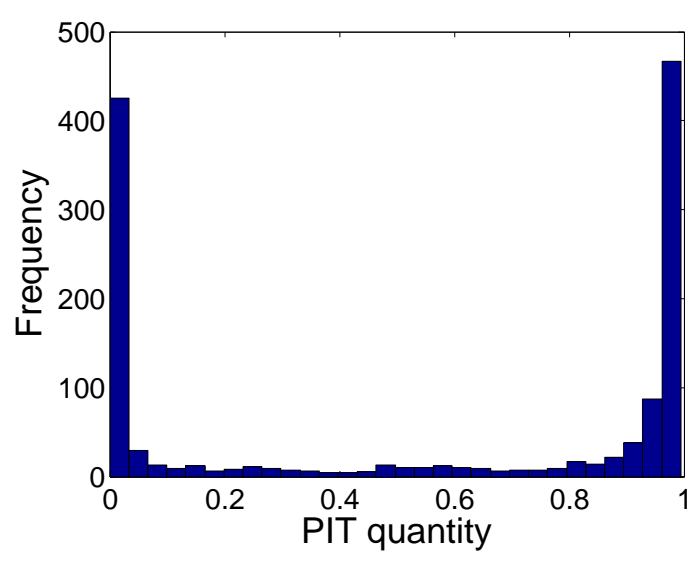

(f) Poisson: TEVA

Figure 6.3: Distribution of the PIT quantities when constrained to $n=p=2, \boldsymbol{\eta}(0)=\mathbf{0}$, $\eta_{11}(1)=\eta_{22}(1), \eta_{12}(1)=\eta_{21}(1), \beta_{1}=\beta_{2}, \theta_{1}=\theta_{2}$, and using Algorithm I for the 2:00-3:00 window. 


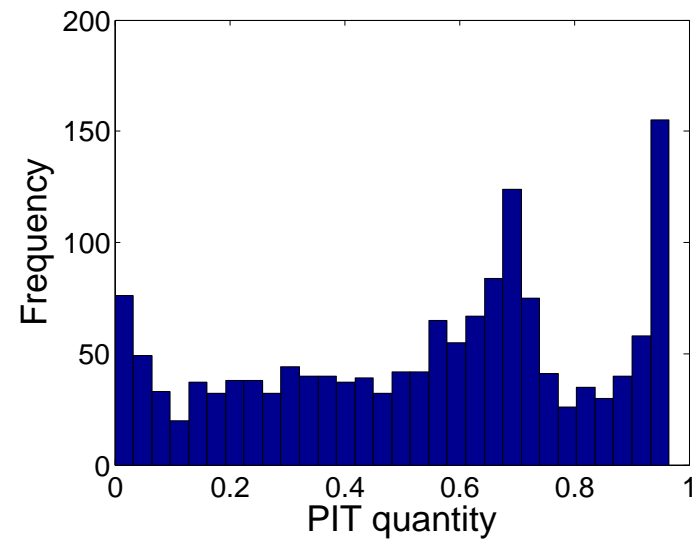

(a) Generalized Hawkes: BBBY

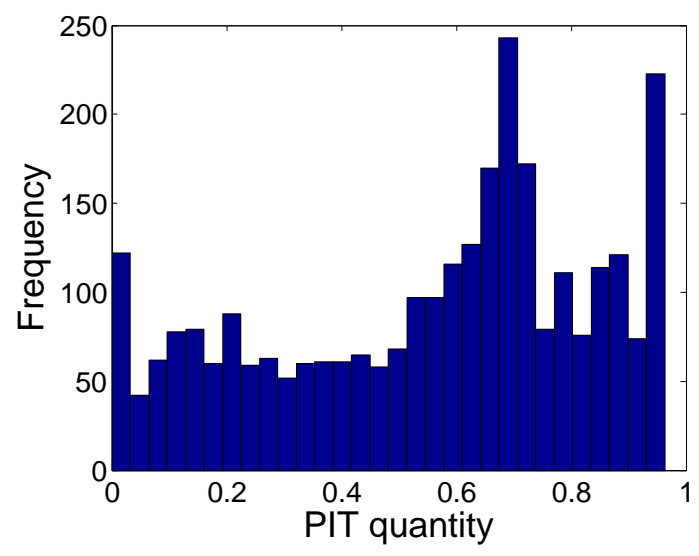

(c) Generalized Hawkes: MSFT

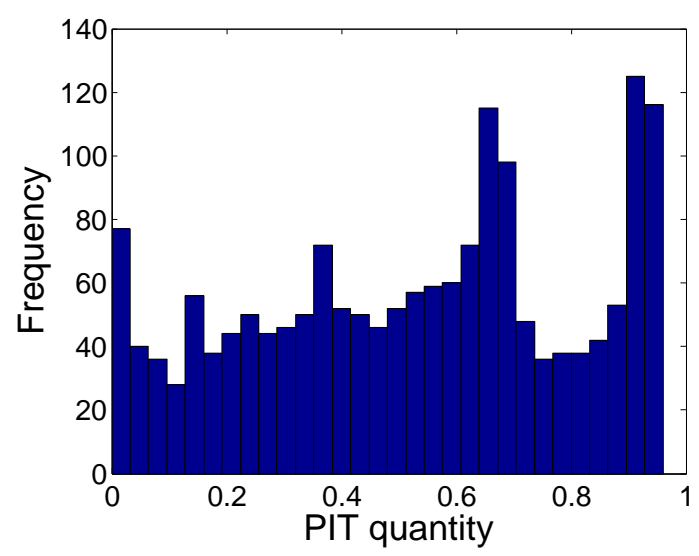

(e) Generalized Hawkes: TEVA

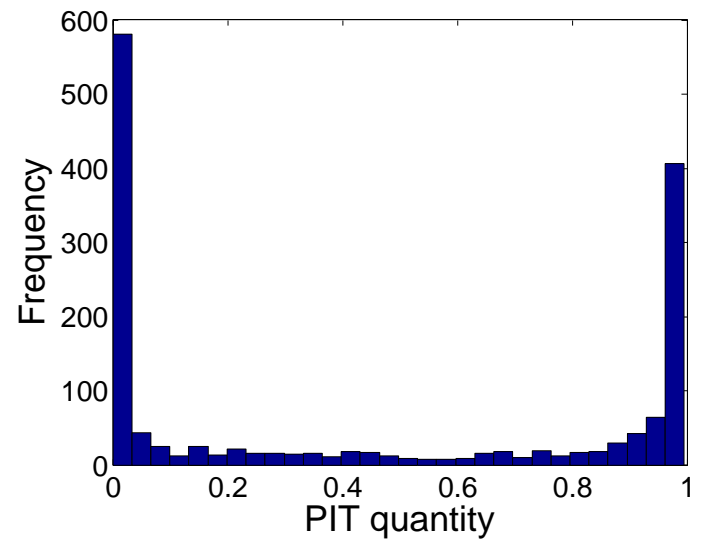

(b) Poisson: BBBY

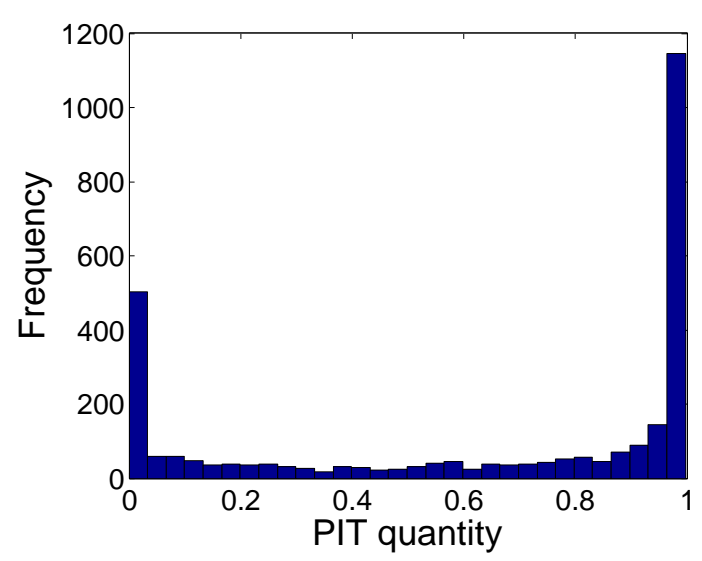

(d) Poisson: MSFT

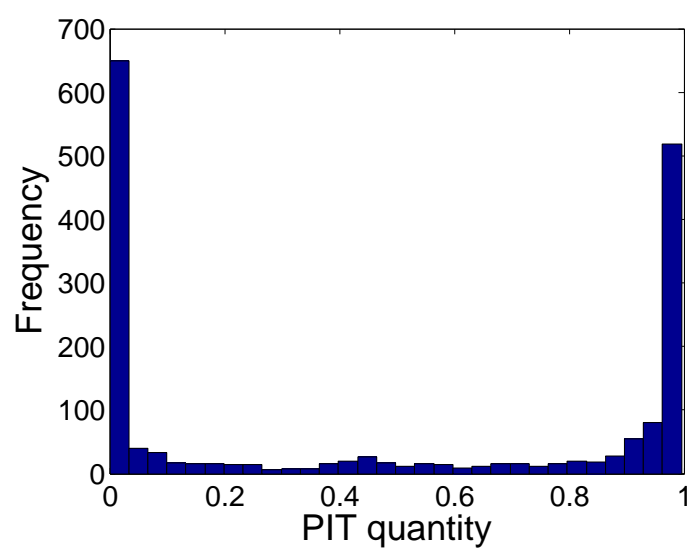

(f) Poisson: TEVA

Figure 6.4: Distribution of the PIT quantities when constrained to $n=p=2, \boldsymbol{\eta}(0)=\mathbf{0}$, $\eta_{11}(1)=\eta_{22}(1), \eta_{12}(1)=\eta_{21}(1), \beta_{1}=\beta_{2}, \theta_{1}=\theta_{2}$, and using Algorithm I for the 3:00-4:00 window. 


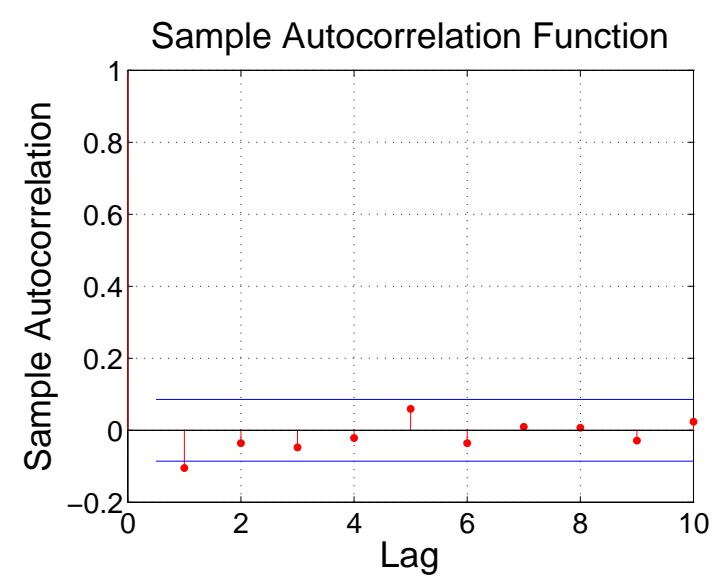

(a) Generalized Hawkes: BBBY

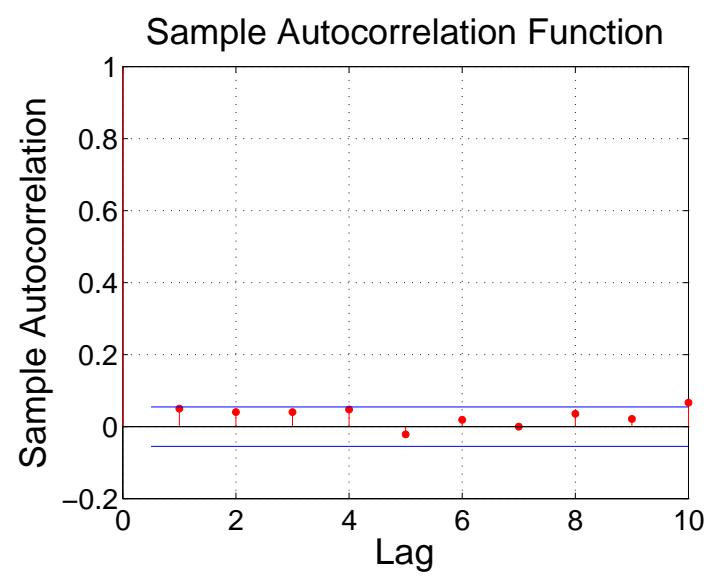

(c) Generalized Hawkes: MSFT

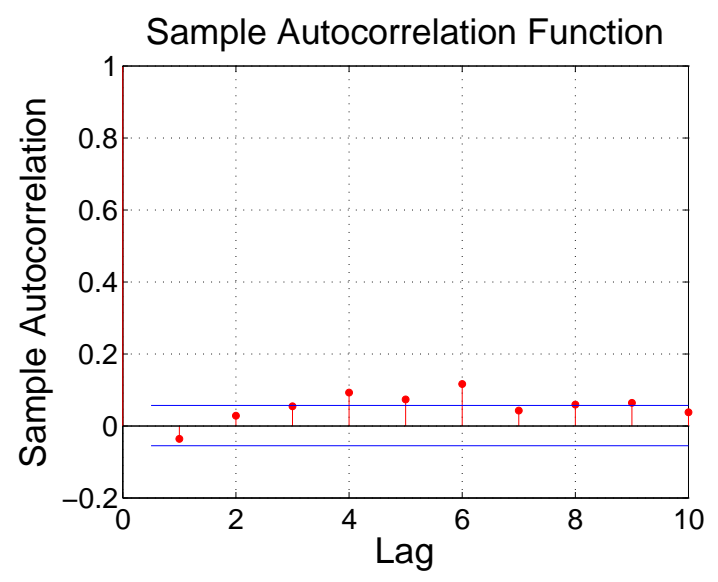

(e) Generalized Hawkes: TEVA

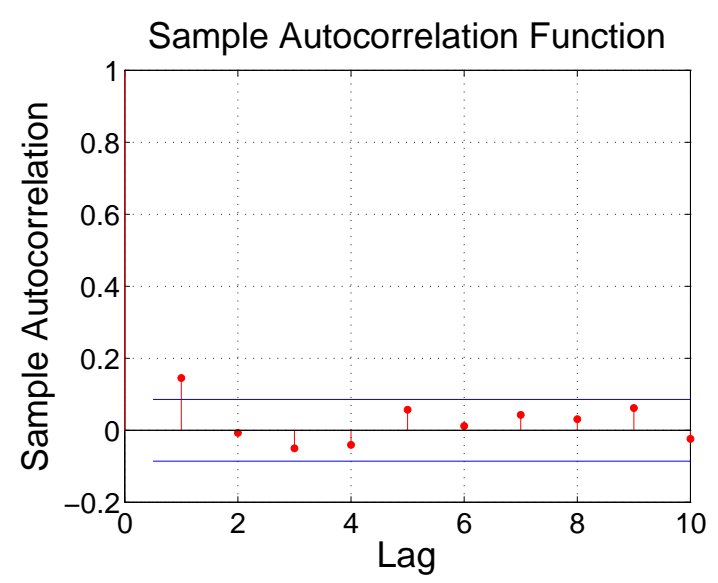

(b) Poisson: BBBY

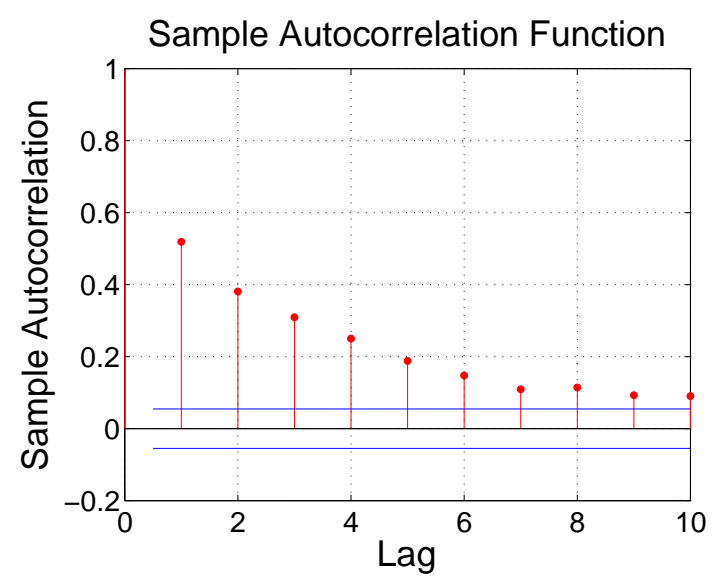

(d) Poisson: MSFT

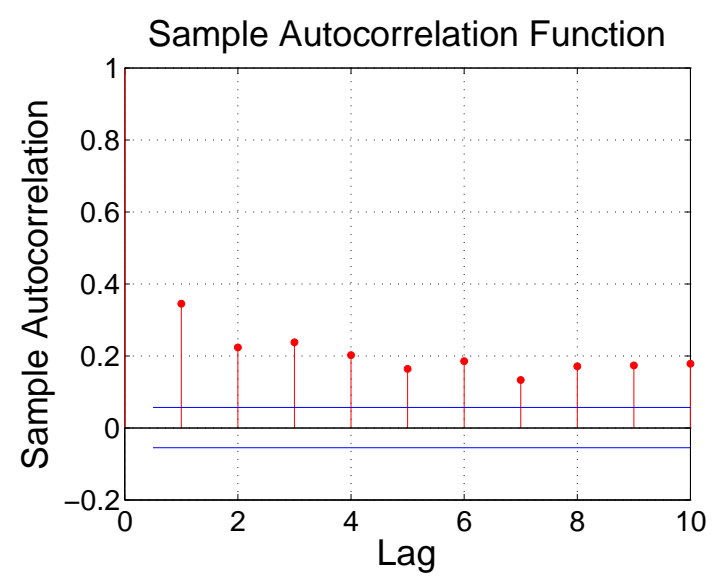

(f) Poisson: TEVA

Figure 6.5: Autocorrelation function of the PIT quantities when constrained to $n=p=2$, $\boldsymbol{\eta}(0)=\mathbf{0}, \eta_{11}(1)=\eta_{22}(1), \eta_{12}(1)=\eta_{21}(1), \beta_{1}=\beta_{2}, \theta_{1}=\theta_{2}$, and using Algorithm I for the 2:00-3:00 window. The blue lines indicate 95\% confidence intervals. 


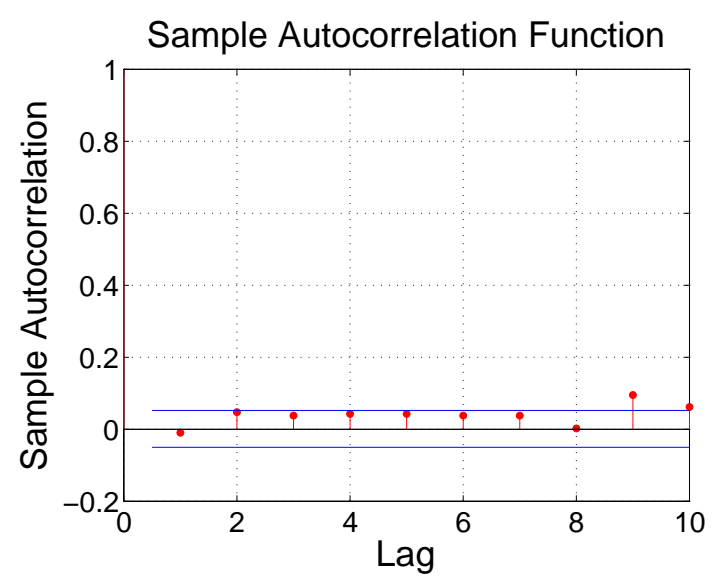

(a) Generalized Hawkes: BBBY

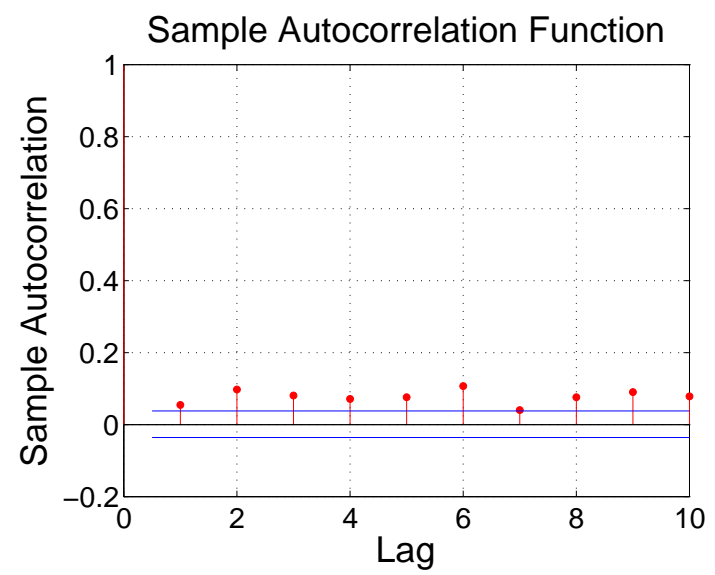

(c) Generalized Hawkes: MSFT

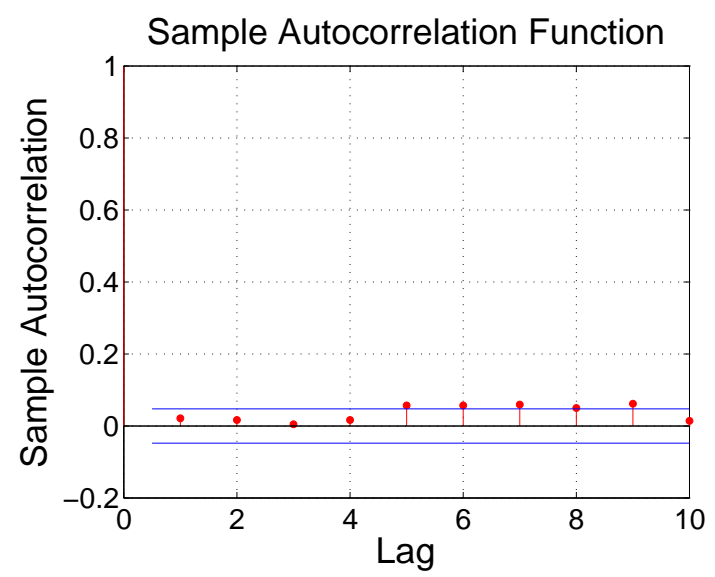

(e) Generalized Hawkes: TEVA

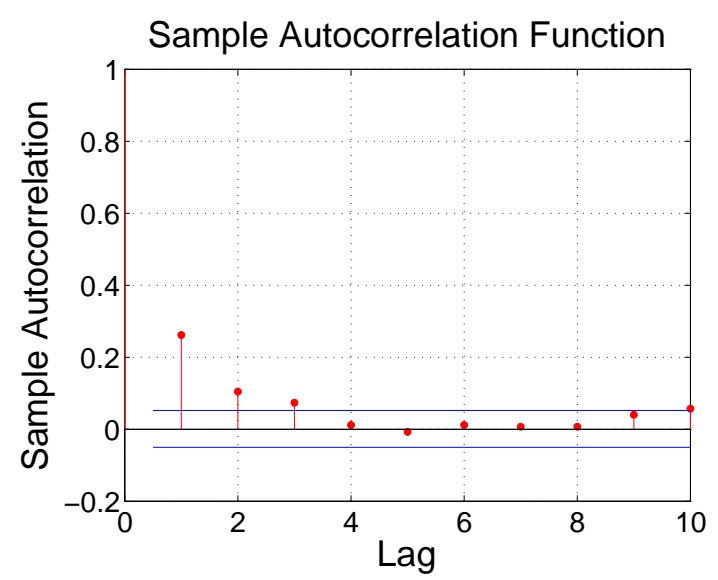

(b) Poisson: BBBY

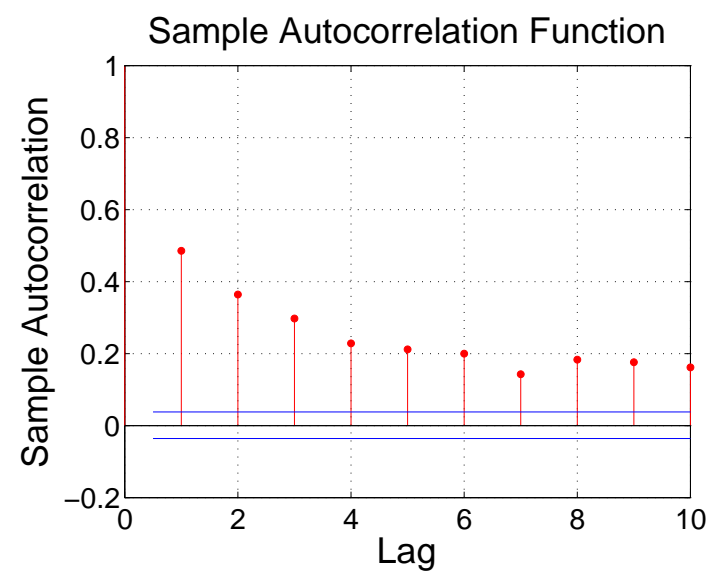

(d) Poisson: MSFT

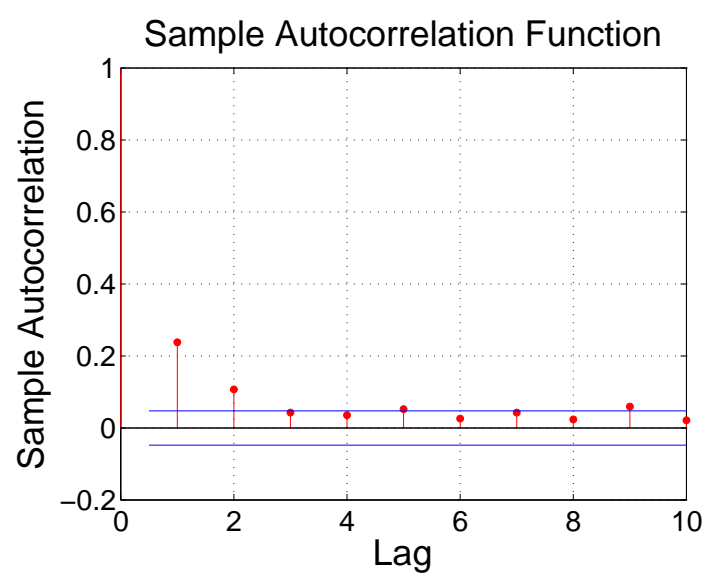

(f) Poisson: TEVA

Figure 6.6: Autocorrelation function of the PIT quantities when constrained to $n=p=2$, $\boldsymbol{\eta}(0)=\mathbf{0}, \eta_{11}(1)=\eta_{22}(1), \eta_{12}(1)=\eta_{21}(1), \beta_{1}=\beta_{2}, \theta_{1}=\theta_{2}$, and using Algorithm I for the 3:00-4:00 window. The blue lines indicate $95 \%$ confidence intervals. 


\subsubsection{Model Validation: Probability-Probability Plots}

By the theory of point processes, it is well known that for any point process $\left\{P_{t}\right\}_{t \geq 0}$ with unique points and $\lim _{t \rightarrow \infty} P_{t}=\infty$, there exists a random time change $t \longmapsto \tau(t)$ such that $\left\{P_{\tau(t)}\right\}_{t \in \mathbb{R}^{+}}$is a Poisson Process with unit intensity (see Daley and Vere-Jones (2007)). In this section, we will perform a random time-change and construct probability-probability (P-P) $\operatorname{plots}^{55}$ for the time-changed process as a diagnostic for model appropriateness.

In the classical $\rho=1$ case, the intensity process $\boldsymbol{\lambda}_{t}$ is observable. In this case, to convert $\left\{N_{t}^{-}+N_{t}^{+}\right\}_{t}$ into a Poisson Process, we know exactly what this random time change should be and is given by

$$
T_{i} \longmapsto \int_{0}^{T_{i}}\left(\lambda_{s}^{-}+\lambda_{s}^{+}\right) d s
$$

However, for the full model, $\boldsymbol{\lambda}_{t}$ is not $\overline{\mathcal{F}}_{t}$ measurable. So we must estimate it given the data. We propose the following random time change for the purpose of generating a P-P plot:

$$
T_{i} \longmapsto \int_{0}^{T_{i}}\left(\hat{\lambda}_{s}^{-}+\hat{\lambda}_{s}^{+}\right) d s
$$

where $\hat{\boldsymbol{\lambda}}_{t}$ is taken to be the mean of the $\overline{\mathcal{F}}_{t_{i-1}}$ measurable particles at time $t$ (propagated forward with exponential decay). The reason that we do not want to take $\hat{\boldsymbol{\lambda}}_{t}$ as the mean of the $\overline{\mathcal{F}}_{t}$-measurable particles is because we do not want $\hat{\boldsymbol{\lambda}}_{t}$, for $t \in\left(t_{i-1}, t_{i}\right)$, to contain any information about $T_{i}$.

Figure 6.7 is a comparison of P-P plots between the classical Hawkes model $(\rho=1)$, the full model, and the Poisson model. The jump sizes in the classical Hawkes case are scaled down to reflect the increase in $\rho$ to $\rho=1$ (the mean jump size and long run mean activity rates are both preserved).

Notice how the time changed quantiles of the full model better track the exponential quantiles. Divergence of dense clusters of points in the P-P plot from the $45^{\circ}$-line can be interpreted as model inadequacy, and even more so when the P-P plot possesses discernable curvature. Such patterns are experienced throughout the collected data. These plots continue to expose the drastic inadequacy of the Poisson model over the classical Hawkes model, and further supports the choice for a model that is more general than the classical Hawkes.

\footnotetext{
${ }^{55} \mathrm{~A}$ P-P plot compares the empirical cumulative distribution function (CDF) of a dataset with a specified theoretical CDF.
} 


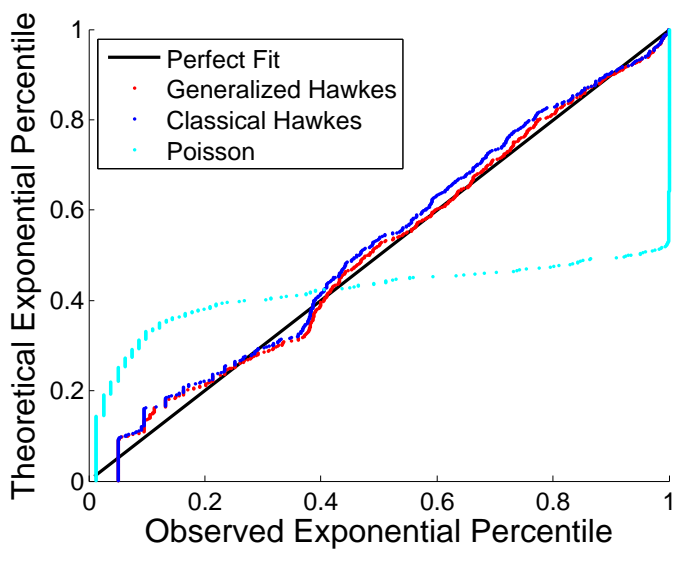

(a) BBBY - 2:00-3:00

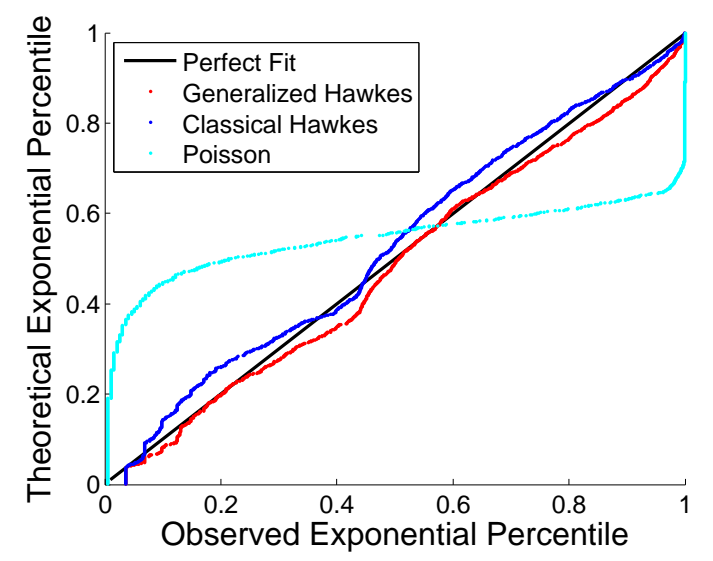

(c) MSFT - 2:00-3:00

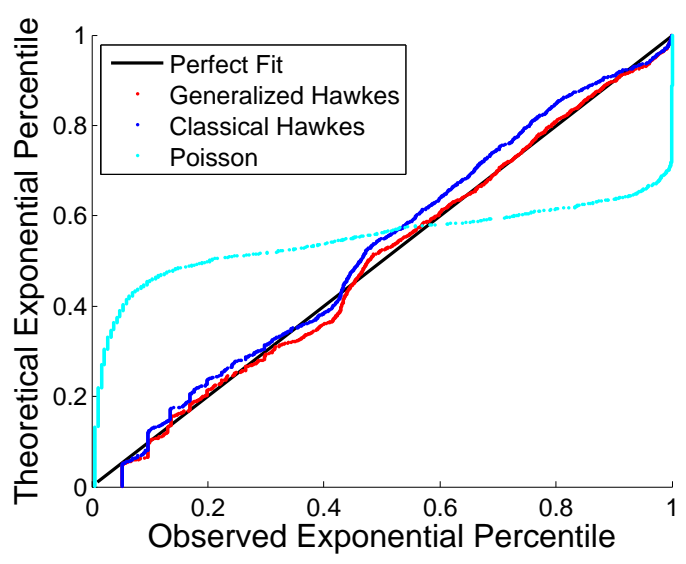

(e) TEVA - 2:00-3:00

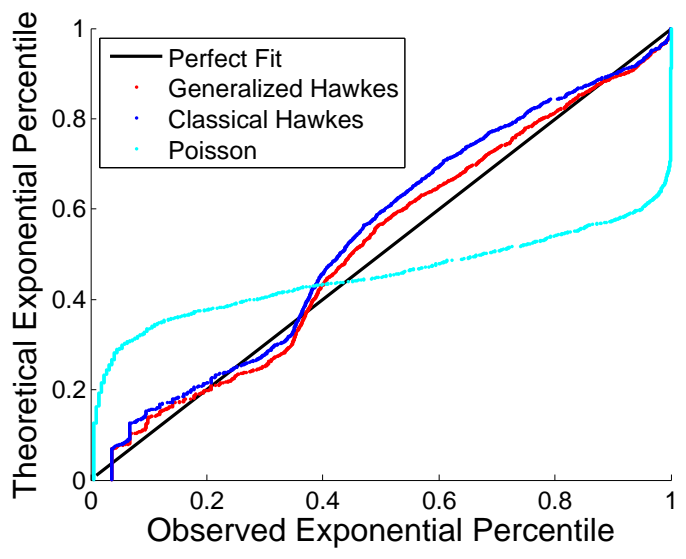

(b) BBBY - 3:00-4:00

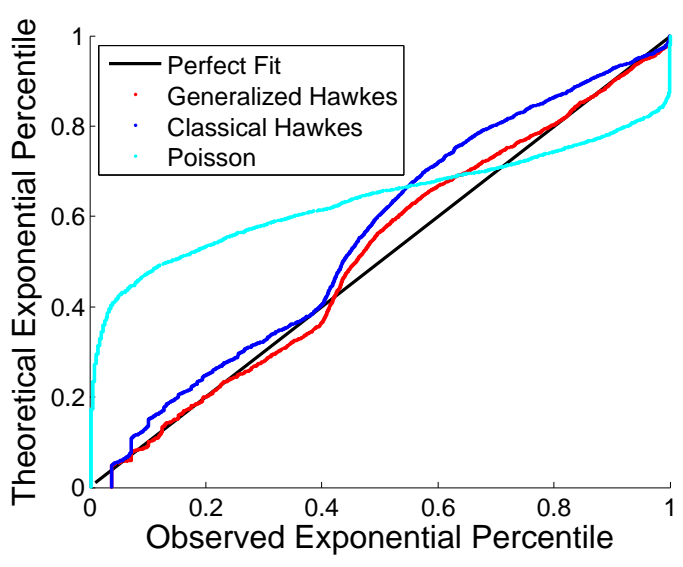

(d) MSFT - 3:00-4:00

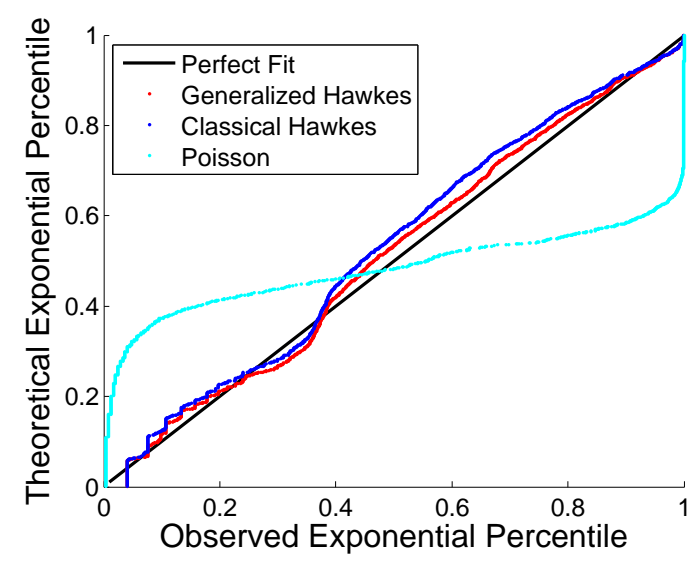

(f) TEVA - 3:00-4:00

Figure 6.7: Exponential P-P plots when constrained to $n=p=2, \boldsymbol{\eta}(0)=\mathbf{0}, \eta_{11}(1)=$ $\eta_{22}(1), \eta_{12}(1)=\eta_{21}(1), \beta_{1}=\beta_{2}, \theta_{1}=\theta_{2}$, and using Algorithm I. 


\subsection{Statistical Results: Generalized Latent Hawkes Process when $\boldsymbol{\eta}(0) \geq 0$}

In this section, we apply Algorithm II, as described in Section 5.4.2, to the symmetric and non-symmetric 2-dimensional Generalized Latent Hawkes model. This differs from the statistical analysis in Section 6.1 in that we do not impose the constraint $\boldsymbol{\eta}(0)=\mathbf{0}$ (recall that the inequality $\boldsymbol{\eta}(0) \geq \mathbf{0}$ should be interpreted element-wise). To ensure identifiability and numerical stability (as discussed in Section 5.3.2), we also impose that each element of $|\boldsymbol{\eta}(1)-\boldsymbol{\eta}(0)|$, as well as $\rho$ and $1-\rho$, are larger than some positive constant (0.01 in this case).$^{56}$

As in Section 6.1.1, we perform a calibration to disjoint 60 minute windows between 10:00am and 4:00pm for each day in May 2011. The analysis is performed for the three tickers BBBY, MSFT, and TEVA. See Tables 6.6 and 6.7 for the means and standard deviations (taken over all 60-minute windows) of parameter estimates. Notice that the elements of $\boldsymbol{\eta}(0)$ not being statistically different from zero, in addition to the elements of $\boldsymbol{\eta}(0)$ being small relative to $\boldsymbol{\eta}(1)$, suggests that the constraint $\boldsymbol{\eta}(0)=\mathbf{0}$ imposed on the data in Section 6.1 may in fact be a valid one. Although one can compute p-values to test such a hypothesis, the fact that the true model is not an element of the parameter space will certainly skew the results of such a test.

Again, there is little qualitative difference between the pairs of parameters associated with symmetry (i.e. $\left(\beta_{1}, \beta_{2}\right),\left(\eta_{12}, \eta_{21}\right)$, etc). As a parallel to the analysis in Section 6.1.1, Table 6.8 provides the relevant empirical correlations for confirming that these pairs of estimators are not negatively correlated.

Finally, to investigate the stability of the parameter estimates, histograms depicting their empirical distributions are provided in Figure 6.8. As the purpose of this section is simply to display Algorithm II producing meaningful estimators on real data (where Algorithm I would fail to do so), goodness of fit analysis has been omitted.

\footnotetext{
${ }^{56}$ The additional constraint on $|\boldsymbol{\eta}(1)-\boldsymbol{\eta}(0)|$ is to avoid the case where they are equal and $\rho$ would not be unique. The additional constraint on $\rho$ is to avoid the case when $\boldsymbol{\eta}(k)$ has zero probability for some $k$. Both of these scenarios would result in a non-identifiable model.
} 


\begin{tabular}{crrrrrrrr}
\hline \hline & \multicolumn{2}{c}{ BBBY } & & \multicolumn{2}{c}{ MSFT } & & \multicolumn{2}{c}{ TEVA } \\
\cline { 2 - 3 } \cline { 8 - 9 } & mean & $($ std $)$ & & mean & $($ std $)$ & & mean & $($ std $)$ \\
\hline$\widehat{\rho}$ & 0.755 & $(0.205)$ & & 0.451 & $(0.102)$ & & 0.747 & $(0.236)$ \\
$\widehat{\beta}$ & 96.69 & $(23.56)$ & & 85.58 & $(27.04)$ & & 107.49 & $(26.56)$ \\
$\widehat{\eta}_{11}(0)$ & 7.96 & $(7.97)$ & & 2.81 & $(3.59)$ & & 8.67 & $(9.85)$ \\
$\widehat{\eta}_{11}(1)$ & 44.81 & $(16.44)$ & & 86.67 & $(24.47)$ & & 55.54 & $(18.08)$ \\
$\widehat{\eta}_{12}(0)$ & 3.05 & $(2.27)$ & & 2.20 & $(2.66)$ & & 4.58 & $(3.85)$ \\
$\widehat{\eta}_{12}(1)$ & 5.48 & $(2.25)$ & & 12.60 & $(4.53)$ & & 9.52 & $(3.78)$ \\
$\widehat{\theta}$ & 0.081 & $(0.034)$ & & 0.156 & $(0.061)$ & & 0.107 & $(0.050)$ \\
\hline $\mathbb{E}\left[\eta_{11} \mid \widehat{\Theta}\right]$ & 31.89 & $(8.62)$ & & 39.12 & $(14.12)$ & & 39.19 & $(12.76)$ \\
$\mathbb{E}\left[\eta_{12} \mid \widehat{\Theta}\right]$ & 4.04 & $(1.64)$ & & 5.60 & $(2.12)$ & & 6.72 & $(2.52)$ \\
$L R M$ & 0.136 & $(0.056)$ & & 0.356 & $(0.151)$ & & 0.203 & $(0.098)$ \\
\hline \hline
\end{tabular}

Table 6.6: Mean and standard deviations of estimators on historical data when $n=p=2$, $\eta_{11}(1)=\eta_{22}(1), \eta_{12}(1)=\eta_{21}(1), \beta_{1}=\beta_{2}, \theta_{1}=\theta_{2}$, and using Algorithm II with 200 particles. Total number of free parameters is 7. LRM refers to the long run mean of activity rate of any component of $\mathbf{N}_{t}$. Sample consists of disjoint 60-minute windows between 10:00am and 4:00pm from May 2011. 


\begin{tabular}{|c|c|c|c|c|c|c|}
\hline & \multicolumn{2}{|c|}{ BBBY } & \multicolumn{2}{|c|}{ MSFT } & \multicolumn{2}{|c|}{ TEVA } \\
\hline & mean & (std) & mean & (std) & mean & (std) \\
\hline$\widehat{\rho}$ & 0.769 & $(0.201)$ & 0.460 & $(0.085)$ & 0.749 & $(0.211)$ \\
\hline$\widehat{\beta}_{1}$ & 94.71 & $(30.13)$ & 87.24 & $(30.07)$ & 106.18 & $(29.35)$ \\
\hline$\widehat{\beta}_{2}$ & 102.50 & $(30.76)$ & 88.43 & $(31.67)$ & 113.54 & (33.37) \\
\hline$\widehat{\eta}_{11}(0)$ & 6.42 & $(5.99)$ & 2.19 & $(2.60)$ & 5.65 & (6.89) \\
\hline$\widehat{\eta}_{11}(1)$ & 43.47 & $(16.41)$ & 87.01 & $(27.78)$ & 57.19 & $(20.27)$ \\
\hline$\widehat{\eta}_{12}(0)$ & 2.38 & $(1.84)$ & 1.20 & $(1.65)$ & 3.67 & ( 3.28$)$ \\
\hline$\widehat{\eta}_{12}(1)$ & 5.70 & $(2.87)$ & 13.88 & $(5.81)$ & 9.63 & ( 4.12$)$ \\
\hline$\widehat{\eta}_{21}(0)$ & 2.57 & $(1.56)$ & 1.23 & $(1.82)$ & 3.94 & ( 3.31$)$ \\
\hline$\widehat{\eta}_{21}(1)$ & 5.35 & $(2.39)$ & 12.68 & $(5.32)$ & 9.39 & $(4.46)$ \\
\hline$\widehat{\eta}_{22}(0)$ & 5.61 & $(6.02)$ & 3.14 & $(3.48)$ & 5.82 & ( 6.17$)$ \\
\hline$\widehat{\eta}_{22}(1)$ & 46.41 & $(20.27)$ & 86.51 & $(27.75)$ & 55.12 & (20.67) \\
\hline$\widehat{\theta}_{1}$ & 0.083 & $(0.035)$ & 0.166 & $(0.080)$ & 0.112 & $(0.055)$ \\
\hline$\widehat{\theta}_{2}$ & 0.082 & $(0.034)$ & 0.151 & $(0.057)$ & 0.109 & $(0.051)$ \\
\hline $\mathbb{E}\left[\eta_{11} \mid \widehat{\Theta}\right]$ & 31.79 & $(10.11)$ & 40.07 & $(15.02)$ & 40.44 & (11.98) \\
\hline $\mathbb{E}\left[\eta_{12} \mid \widehat{\Theta}\right]$ & 4.32 & $(2.33)$ & 6.33 & $(2.74)$ & 7.02 & $(3.27)$ \\
\hline $\mathbb{E}\left[\eta_{21} \mid \widehat{\Theta}\right]$ & 4.05 & $(1.86)$ & 5.73 & $(2.38)$ & 6.79 & $(3.12)$ \\
\hline $\mathbb{E}\left[\eta_{22} \mid \widehat{\Theta}\right]$ & 33.08 & $(10.77)$ & 39.73 & $(14.74)$ & 38.95 & (12.18) \\
\hline$L R M_{1}$ & 0.139 & $(0.060)$ & 0.373 & $(0.183)$ & 0.211 & $(0.105)$ \\
\hline$L R M_{2}$ & 0.134 & $(0.056)$ & 0.347 & $(0.144)$ & 0.197 & $(0.096)$ \\
\hline
\end{tabular}

Table 6.7: Mean and standard deviations of estimators on historical data when $n=p=2$ and using Algorithm II with 200 particles. Total number of free parameters is 13. Sample consists of disjoint 60-minute windows between 10:00am and 4:00pm from May 2011.

\begin{tabular}{cccc}
\hline \hline & BBBY & MSFT & TEVA \\
\hline $\operatorname{corr}\left(\widehat{\beta}_{1}, \widehat{\beta}_{2}\right)$ & 0.184 & 0.518 & 0.264 \\
$\operatorname{corr}\left(\widehat{\eta}_{11}(0), \widehat{\eta}_{22}(0)\right)$ & 0.510 & 0.018 & 0.291 \\
$\operatorname{corr}\left(\widehat{\eta}_{11}(1), \widehat{\eta}_{22}(1)\right)$ & 0.399 & 0.554 & 0.411 \\
$\operatorname{corr}\left(\widehat{\eta}_{12}(0), \widehat{\eta}_{21}(0)\right)$ & 0.147 & 0.250 & 0.294 \\
$\operatorname{corr}\left(\widehat{\eta}_{12}(1), \widehat{\eta}_{21}(1)\right)$ & 0.161 & 0.476 & 0.310 \\
$\operatorname{corr}\left(\widehat{\theta}_{1}, \widehat{\theta}_{2}\right)$ & 0.844 & 0.663 & 0.791 \\
\hline \hline
\end{tabular}

Table 6.8: Sample correlation of seletced pairs of estimators when the calibration does not impose symmetry for general $\boldsymbol{\eta}(0) \geq \mathbf{0}$. 


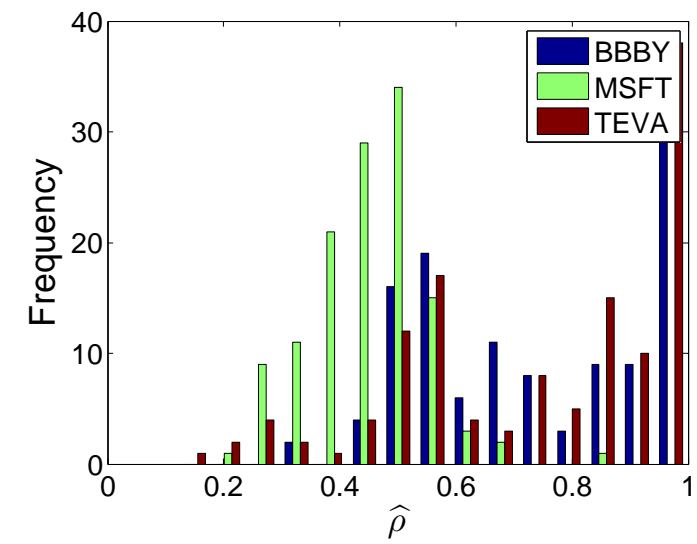

(a) Frequency of $\widehat{\rho}$

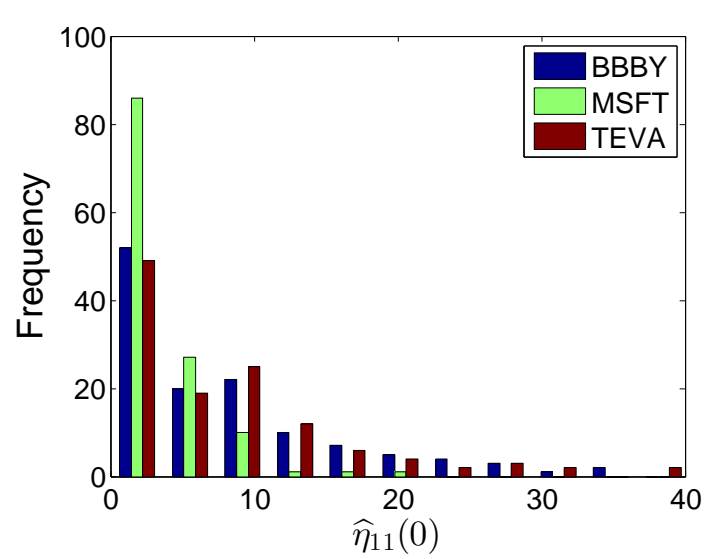

(c) Frequency of $\widehat{\eta}_{11}(0)$

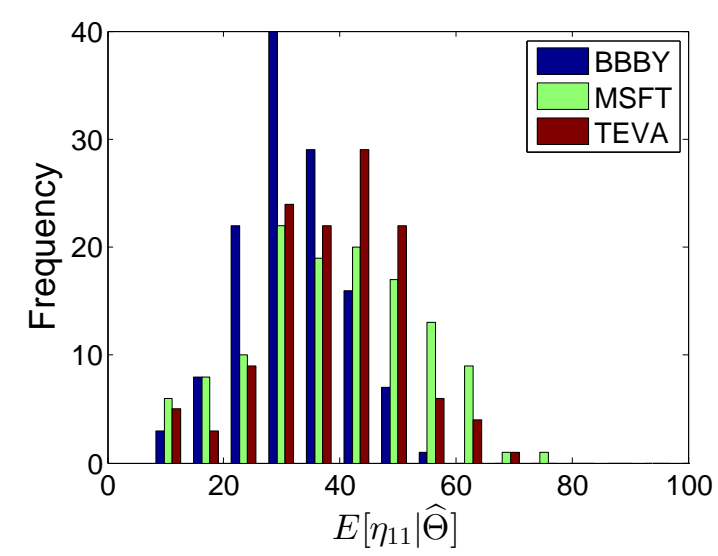

(e) Frequency of $\mathbb{E}\left(\eta_{11} \mid \widehat{\Theta}\right)$

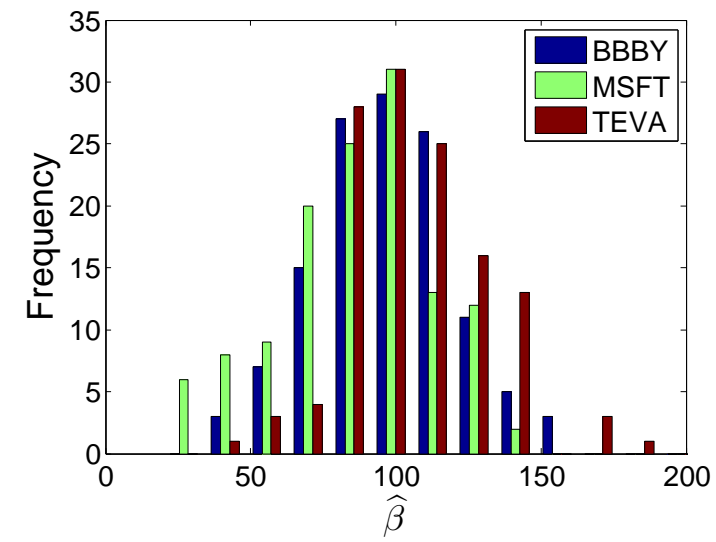

(b) Frequency of $\widehat{\beta}$

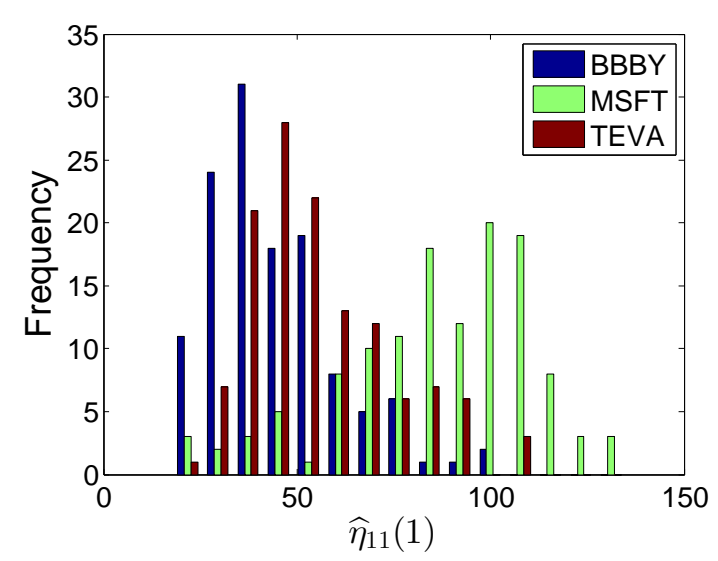

(d) Frequency of $\widehat{\eta}_{11}(1)$

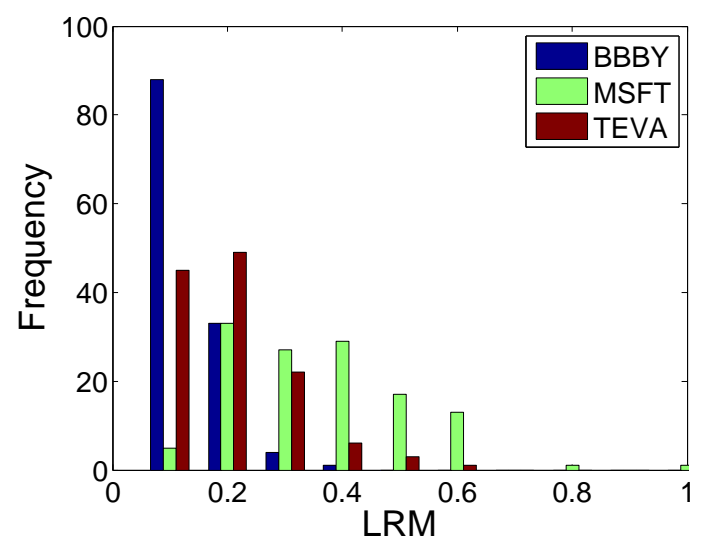

(f) Frequency of LRM

Figure 6.8: Frequency of parameter estimates when constrained to $n=p=2, \eta_{11}=\eta_{22}$, $\eta_{12}=\eta_{21}, \beta_{1}=\beta_{2}, \theta_{1}=\theta_{2}$, and using Algorithm II. 


\subsection{Algorithmic Trading in a Single Asset}

Recall that the market maker in Chapter 3 solves the stochastic control problem

$$
\Phi(t, x, s, q, \alpha, \boldsymbol{\lambda}, \boldsymbol{\kappa})=\sup _{\left(\delta_{u}^{-}, \delta_{u}^{+}\right)_{t \leq u \leq T} \in \mathcal{A}} \mathbb{E}_{(t, x, s, q, \alpha, \boldsymbol{\lambda}, \boldsymbol{\kappa})}\left[X_{T}+q_{T} S_{T}-\phi \int_{t}^{T} q_{s}^{2} d s\right]
$$

where $x$ is her cash reserve, $s$ is the asset's midprice, $q$ is her current inventory, $\alpha$ is the short-term drift in the asset's midprice, $\boldsymbol{\lambda}$ is the MO intensity function, $\boldsymbol{\kappa}$ is the shape parameter for the depth of the LOB, and we are optimizing over LO quote depths $\delta^{ \pm}$.

The optimal market making strategy, as stated in Corollary 3.5.3, is given by

$$
\delta^{ \pm *}=\delta_{0}^{ \pm}+B\left(\delta_{0}^{ \pm} ; \boldsymbol{\kappa}^{ \pm}\right)\left\{ \pm \mathbb{S}_{\lambda}^{ \pm}\left(\mathbb{E}_{t}\left[\int_{t}^{T} \alpha_{u} d u\right]\right)+\phi\left( \pm \mathbb{S}_{\lambda}^{ \pm} b_{\phi}+(1 \mp 2 q)(T-t)\right)\right\}
$$

with explicit expressions given for each term in Section 3.5.3. Recall that, in addition to the risk-neutral LO quote $\delta_{0}^{ \pm}$, the optimal spreads have explicit dependence on the expected integrated drift $\mathbb{E}_{t}\left[\int_{t}^{T} \alpha_{u} d u\right]$, the imbalance in the rates of incoming MOs via $\phi b_{\phi}$, and the inventory control term $\phi(1 \mp 2 q)$.

We use the parameter estimates from Table 6.9 (Sample period is Jan-Apr 2011), in conjunction with our SMC particle filtering methods of Section 5.5.2, to test the effectiveness of this single asset marking making strategy on real data ${ }^{57}$ from May 2011 . The per-second sample standard deviation (using all daily price differences for the stated calibration period and rescaling appropriately) is also reported. ${ }^{58}$ Observe that if were to use the parameter estimates from Table 6.1 in this strategy, then this would be considered in sample testing and would violate the condition that any strategy should be adapted to $\overline{\mathcal{F}}_{t}$. This distinction (between using the mean of Jan-May versus Jan-Apr estimators), however, does not have any material impact on results (compare Tables 6.1 and 6.9).

The high frequency trader (HFT) will make markets in 3 different equities (BBBY, MSFT, and TEVA - treated individually ${ }^{59}$ between 10:00am and 3:30pm on each business day. She will also be required to be flat $(q=0)$ at each 5 minute interval. Because of this condition, the HFT sets $T=300$ seconds as the trading horizon in the optimal strategy

\footnotetext{
${ }^{57}$ The backtesting of this strategy requires Level II financial data because we require knowledge of the entire limit order book.

${ }^{58}$ Because using an incorrect value for $\sigma$ is equivalent to using a different inventory-aversion level $\phi$, we only seek an approximate estimate of $\sigma$. In fact, recall that the optimal limit quote posting depth $\delta_{t}^{ \pm}$is free of $\sigma$ (see Corollary 3.5.3). Hence, $\widehat{\sigma}$ is only used when calculating mean inventory risk.

${ }^{59}$ Backtesting for BBBY and MSFT was performed on data from the NASDAQ exchange. Backtesting for TEVA was performed on data from the NYSE exchange.
} 


\begin{tabular}{crrrrrrrr}
\hline \hline & \multicolumn{2}{c}{ BBBY } & & \multicolumn{2}{c}{ MSFT } & & \multicolumn{2}{c}{ TEVA } \\
\cline { 2 - 3 } \cline { 8 - 9 } \cline { 8 - 9 } & mean & $($ std $)$ & & mean & $($ std $)$ & & mean & (std) \\
\hline$\widehat{\rho}$ & 0.768 & $(0.173)$ & & 0.410 & $(0.089)$ & & 0.770 & $(0.167)$ \\
$\widehat{\beta}$ & 94.41 & $(26.99)$ & & 106.27 & $(26.90)$ & & 115.20 & $(34.39)$ \\
$\widehat{\eta}_{11}(1)$ & 49.09 & $(16.51)$ & & 127.33 & $(31.13)$ & & 61.35 & $(19.28)$ \\
$\widehat{\eta}_{12}(1)$ & 6.63 & $(2.36)$ & & 19.68 & $(6.63)$ & & 12.12 & $(4.35)$ \\
$\widehat{\theta}$ & 0.086 & $(0.043)$ & & 0.158 & $(0.078)$ & & 0.104 & $(0.055)$ \\
$\widehat{\sigma}$ & 0.0039 & - & 0.0018 & - & & 0.0036 & - \\
\hline \hline
\end{tabular}

Table 6.9: Fitted parameters to be used in the backtesting of our single asset market making strategy. Sample period is Jan-Apr 2011.

\begin{tabular}{cc}
\hline \hline Parameter & Value \\
\hline$\kappa_{\beta}$ & 10 \\
$\kappa_{\eta}$ & 10 \\
$\kappa_{\nu}$ & 25 \\
$\kappa_{\theta}$ & 50 \\
$\zeta$ & 2 \\
$\sigma_{\alpha}$ & 0.01 \\
$\epsilon$ & 0.04 \\
$T$ & 300 \\
lot size & 100 shares \\
\hline \hline
\end{tabular}

Table 6.10: Other model parameters to be used in the backtesting of the single asset market making strategy.

given in Corollary 3.5.3. The other parameters in the model are given in Table $6.10 .{ }^{60}$ Note that one unit of inventory will correspond to 100 shares of the equity. Although we simply use the estimators in Table 6.9 over the entire month of May, in practice one would periodically update these estimates using the data from the previous 30-60 minute window. With such a continuous calibration procedure, the strategy's performance may be significantly improved.

\footnotetext{
${ }^{60}$ These are taken to be the same as in the simulation study of Section 3.6 as they provide reasonable estimates for LOB volume and suspected short-term drift behaviour.
} 


\subsubsection{The Inclusion of Market Orders}

As demonstrated in Section 2.4, the ability for the HFT to execute market orders is valuable. This effect is accentuated when the investor faces the strong adverse selection effects present in real financial markets and aggressively wants to take liquidity from the market to acquire a favourable position (or liquidate an unfavourable one).

However, the inclusion of market orders turns this problem into a combined impulse stochastic control problem. The corresponding Hamilton-Jacobi-Bellman equation will then be a Hamilton-Jacobi-Bellman quasi-variational inequality (HJBQVI) of the form of (2.13) and we lose analytic tractability in the asymptotic regime that the problem in Chapter 3 was solved in. Instead of resorting to a numerical scheme to solve the problem (with 5 continuous spatial dimensions in addition to inventory and time), we impose a specific ad-hoc criteria of when to execute market orders to combat adverse selection, as described below.

The investor submits a market buy order at time $t$ whenever the following two conditions are met: $\mathbb{E}\left[\int_{t}^{t+1} \alpha_{u} d u \mid \overline{\mathcal{F}}_{t}\right]>\bar{\alpha}$ (the existence of a sufficiently strong directional signal), where $\bar{\alpha}>0$ is some prespecified threshold and referred to as the investor's MO aggression level, and $\delta_{t}^{-}=0$ (which represents the HFT's willingness to trade that way as it jointly incorporates information about $q_{t}, \boldsymbol{\lambda}_{t}, \boldsymbol{\kappa}_{t}$, and $\alpha_{t}$ ). The financial intuition behind the first condition is that it represents the expected change in the midprice over the next 1 second. The threshold $\bar{\alpha}$ can depend on many things, but it should at least be large enough to cover fees, the bid/offer spread incurred for market order submission, etc. The conditions are similar for the submission of a market sell order with $\mathbb{E}\left[\int_{t}^{t+1} \alpha_{u} d u \mid \overline{\mathcal{F}}_{t}\right]<-\bar{\alpha}$ and $\delta_{t}^{+}=0$

The quantity $\mathbb{E}\left[\int_{t}^{t+1} \alpha_{u} d u \mid \overline{\mathcal{F}}_{t}\right]$ is the expected change in the midprice over the next 1 second and is exactly what the model predicts the mean profit and loss (PnL) of holding one unit of the asset (and liquidating at the midprice) to be. The fact that we use 1 second is not particularly important here. The only requirement is that it should be larger than the $1 / 4$-life ${ }^{61}$ of the effect of any individual event to ensure the horizon is long enough for the investor to realize a large proportion of expected profits by holding an open position. The lifetime of any single instance of the signal will be dictated by $\beta$ and $\zeta$, and once $\left(\alpha_{t}, \boldsymbol{\lambda}_{t}, \boldsymbol{\kappa}_{t}\right)$ returns close to its mean-reversion state, the quantity

\footnotetext{
${ }^{61}$ The choice of $1 / 4$ here is an arbitrary one. It is close enough to 0 so that the investor holds the asset long enough to accumulate this non-zero drift, but not so close to 0 such that the investor is holding this risky position long after the directional movements have dissipated.
} 
$\mathbb{E}\left[\int_{t}^{t+u} \alpha_{s} d s \mid \overline{\mathcal{F}}_{t}\right]$ will (effectively) be 0 for all $u \geq 0$, ensuring that the MO aggression signal only depends on evolution until the first reset to $\left(\alpha_{t}, \boldsymbol{\lambda}_{t}, \boldsymbol{\kappa}_{t}\right) \approx\left(0, \boldsymbol{\theta}, \boldsymbol{\theta}_{\kappa}\right)$.

\subsubsection{Performace of the Strategy}

Daily PnL results are reported for inventory-aversion levels $\phi=10^{-8}, 10^{-7}, 10^{-6}, 10^{-5}$, and MO aggression levels $\bar{\alpha}=0.02,0.05,0.10,0.20$ for each of the three equities BBBY, MSFT, and TEVA, in Tables $6.11,6.12$, and 6.13, respectively. The results presented are on a per day basis and are consistent with those in Section 3.6. That is, increasing the investor's inventory aversion parameter decreases her mean PnL, standard deviation of PnL, and also mean inventory risk $^{62}$ (i.e. she holds less risk throughout the strategy). To give some perspective into the mean inventory risk (MIR) risk metric, note that the strategy that holds $\tilde{q}$ units for the entire strategy would have an MIR of $3000 \tilde{q}^{2}, 670 \tilde{q}^{2}$, and $2600 \tilde{q}^{2}$ for each of the three equities, respectively.

Furthermore, the more aggressive the investor is to submit MOs in an attempt to capture short term alpha (i.e. when $\bar{\alpha}$ is small), the greater her mean PnL will be. Again, this is at the expense of having larger variance and holding more risk throughout the day. Similar to the analysis of inventory aversion level $\phi$, the MO aggression level $\bar{\alpha}$ will ultimately depend on the risk profile of the investor.

To put the results of Tables 6.11 - 6.13 into perspective, we look at the distribution of the maximum inventory held by the investor (for each value of $\bar{\alpha}$ ) for each 5 -minute interval in Figure 6.9. This will give some insight into capital and margin requirements. There is also a maximum inventory level of 20 units (long or short) being imposed to prevent the HFT's inventory from becoming too large (in absolute value). The more frequent occurrence of attaining this maximum inventory constraint for MSFT (relative to BBBY and TEVA) is due to the smaller fitted volatility $\widehat{\sigma}$, as reported in Table 6.9.

It is clear that the less aggressive the investor is to submit MOs (larger $\bar{\alpha}$ ), the smaller her maximum inventory level is. Conversely, the more aggressively the investor submits MOs to capture small deviations of $\alpha_{t}$ from 0 , the larger her maximum inventory level is, which may require more capital to fund such a strategy. In the most conservative case where the investor must fund the entire position without leverage, each of the three strategies would require approximately $\$ 55,000$ in capital. ${ }^{63}$ Given that the capital requirements

\footnotetext{
${ }^{62}$ Recall that inventory risk in the single asset case is defined as $\sigma^{2} \int_{0}^{T} q_{s}^{2} d s$.

${ }^{63}$ This is calculated as the cost to fund either 900 shares in BBBY, 2000 shares in MSFT, or 1000 shares in TEVA, as required by the strategy's accumulated inventory (the distribution of this quantity is shown
} 


\begin{tabular}{|c|c|c|c|c|c|}
\hline \multicolumn{6}{|c|}{ BBBY } \\
\hline & $\bar{\alpha}$ & 0.02 & 0.05 & 0.10 & 0.20 \\
\hline \multirow{3}{*}{$\phi=10^{-8}$} & mean & 79.64 & 73.55 & 12.10 & -2.08 \\
\hline & $(\mathrm{std})$ & $(137.82)$ & $(135.16)$ & $(80.20)$ & $(56.76)$ \\
\hline & (MIR) & $(23,741)$ & $(19,884)$ & $(6,112)$ & $(1,293)$ \\
\hline \multirow{3}{*}{$\phi=10^{-7}$} & mean & 79.30 & 72.78 & 12.01 & -2.18 \\
\hline & $(\mathrm{std})$ & $(136.83)$ & $(134.55)$ & $(80.04)$ & $(56.59)$ \\
\hline & (MIR) & $(23,604)$ & $(19,781)$ & $(6,070)$ & $(1,253)$ \\
\hline \multirow{3}{*}{$\phi=10^{-6}$} & mean & 69.70 & 65.23 & 10.13 & -3.46 \\
\hline & $(\mathrm{std})$ & $(131.70)$ & $(131.21)$ & $(80.07)$ & $(55.90)$ \\
\hline & (MIR) & $(22,125)$ & $(18,746)$ & $(5,850)$ & $(1,240)$ \\
\hline \multirow{3}{*}{$\phi=10^{-5}$} & mean & 45.69 & 46.85 & 10.87 & -4.42 \\
\hline & $(\mathrm{std})$ & $(124.11)$ & $(118.57)$ & $(76.31)$ & $(59.12)$ \\
\hline & (MIR) & $(12,154)$ & $(12,304)$ & $(3,971)$ & $(670)$ \\
\hline
\end{tabular}

Table 6.11: Daily PnL results for BBBY. The asset's daily closing price ranged from approximately $\$ 45$ to $\$ 58$ throughout the trading period.

are more or less equal, it is clear that market making on MSFT was the most profitable, followed by TEVA and BBBY.

The HFT strategy also performs the best for MSFT when looking at the strategy's Sharpe Ratio $^{64}$. Furthermore, it is also the most profitable when considering how much PnL is generated for each unit of MIR held by the investor. For every thousand units of mean inventory risk, the HFT earns approximately $\$ 7$ in mean PnL. Compare this with BBBY and TEVA where this ratio is only about $\$ 3$ and $\$ 4$ per thousand units of MIR, respectively.

in Figure 6.9) when the spot prices were at their maximum values of $\$ 58, \$ 29$, and $\$ 57$, respectively.

${ }^{64}$ The Sharpe Ratio is defined as a trading strategy's mean return above a benchmack (we take zero in this thesis because we are concerned with intraday trading) divided by the standard deviation of return. 


\begin{tabular}{|c|c|c|c|c|c|}
\hline \multicolumn{6}{|c|}{ MSFT } \\
\hline & $\bar{\alpha}$ & 0.02 & 0.05 & 0.10 & 0.20 \\
\hline \multirow{3}{*}{$\phi=10^{-8}$} & mean & 521.46 & 445.13 & 217.40 & -0.62 \\
\hline & $(\operatorname{std})$ & $(232.65)$ & $(231.84)$ & $(189.90)$ & (101.18) \\
\hline & (MIR) & $(71,320)$ & $(62,864)$ & $(36,402)$ & $(10,657)$ \\
\hline \multirow{3}{*}{$\phi=10^{-7}$} & mean & 520.03 & 444.98 & 217.69 & -0.14 \\
\hline & (std) & $(231.88)$ & $(231.18)$ & $(190.04)$ & $(101.30)$ \\
\hline & (MIR) & $(70,351)$ & $(62,466)$ & $(36,388)$ & $(10,636)$ \\
\hline \multirow{3}{*}{$\phi=10^{-6}$} & mean & 493.63 & 432.08 & 217.45 & -0.85 \\
\hline & (std) & $(238.28)$ & $(237.31)$ & (192.24) & (101.35) \\
\hline & (MIR) & $(64,475)$ & $(60,334)$ & $(36,048)$ & $(10,410)$ \\
\hline \multirow{3}{*}{$\phi=10^{-5}$} & mean & 260.70 & 220.48 & 104.38 & -22.85 \\
\hline & (std) & $(175.17)$ & $(170.66)$ & $(156.67)$ & $(93.84)$ \\
\hline & (MIR) & $(34,677)$ & $(35,995)$ & $(22,830)$ & $(6,608)$ \\
\hline
\end{tabular}

Table 6.12: Daily PnL results for MSFT. The asset's daily closing price ranged from approximately $\$ 25$ to $\$ 29$ throughout the trading period. 


\begin{tabular}{|c|c|c|c|c|c|}
\hline \multicolumn{6}{|c|}{ TEVA } \\
\hline & $\bar{\alpha}$ & 0.02 & 0.05 & 0.10 & 0.20 \\
\hline \multirow{3}{*}{$\phi=10^{-8}$} & mean & 194.53 & 188.90 & 13.39 & -44.76 \\
\hline & $(\mathrm{std})$ & $(390.24)$ & $(372.03)$ & $(245.26)$ & $(171.54)$ \\
\hline & (MIR) & $(49,303)$ & $(42,950)$ & $(16,378)$ & $(4,609)$ \\
\hline \multirow{3}{*}{$\phi=10^{-7}$} & mean & 192.29 & 186.87 & 13.39 & -44.76 \\
\hline & (std) & $(388.34)$ & $(368.79)$ & $(245.26)$ & $(171.54)$ \\
\hline & (MIR) & $(49,122)$ & $(42,872)$ & $(16,378)$ & $(4,609)$ \\
\hline \multirow{3}{*}{$\phi=10^{-6}$} & mean & 186.89 & 183.91 & 10.39 & -45.19 \\
\hline & $(\mathrm{std})$ & $(373.56)$ & $(361.22)$ & $(246.54)$ & $(166.90)$ \\
\hline & (MIR) & $(46,390)$ & $(41,065)$ & $(16,181)$ & $(4,331)$ \\
\hline \multirow{3}{*}{$\phi=10^{-5}$} & mean & 60.48 & 68.16 & -34.20 & -53.94 \\
\hline & (std) & $(250.02)$ & $(254.07)$ & $(225.55)$ & (151.91) \\
\hline & (MIR) & $(22,245)$ & $(23,406)$ & $(9,649)$ & $(2,431)$ \\
\hline
\end{tabular}

Table 6.13: Daily PnL results for TEVA. The asset's daily closing price ranged from approximately $\$ 45$ to $\$ 57$ throughout the trading period. 


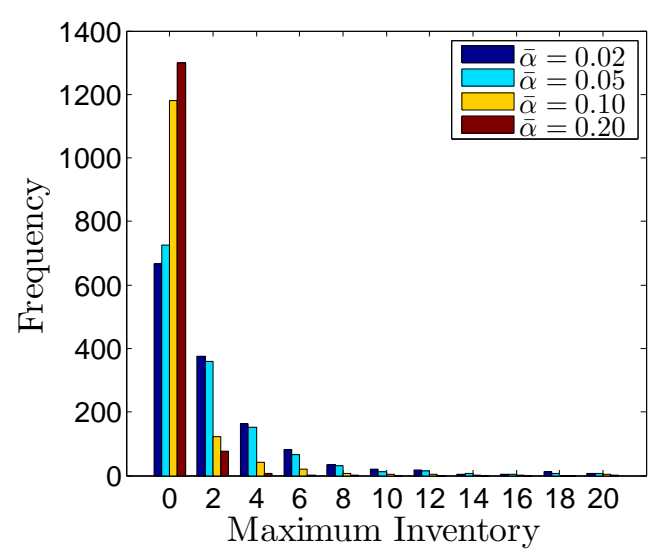

(a) BBBY

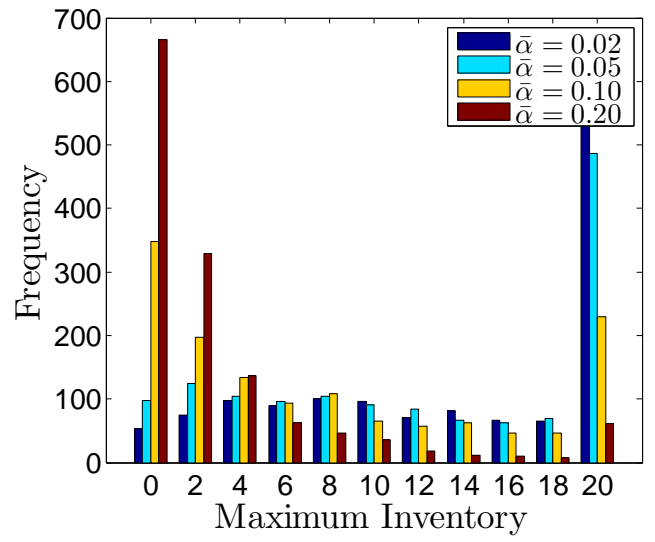

(b) MSFT

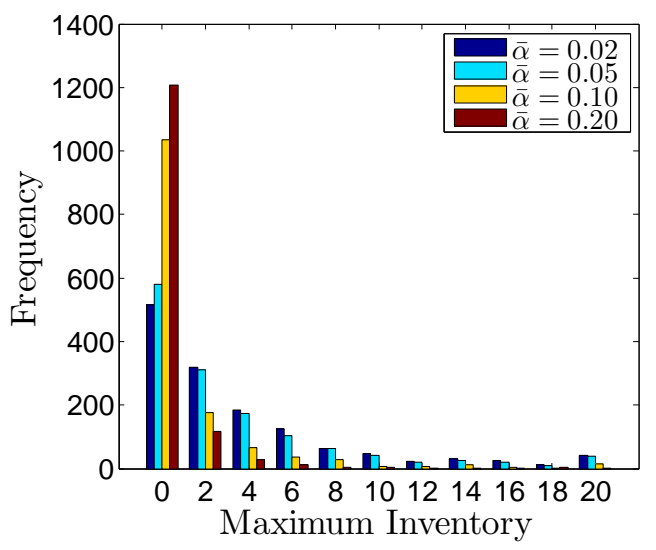

(c) TEVA

Figure 6.9: Distribution of $\max _{0 \leq t \leq T}\left\{\left|q_{t}\right|\right\}$ for various MO aggression levels, $\bar{\alpha}$. The inventory aversion level is fixed at $\phi=10^{-7}$. There are 1,386 5-minute trading intervals in this sample. 


\section{Chapter 7}

\section{Final Conclusions}

\subsection{Summary of Contributions}

This thesis derives optimal high frequency trading strategies for a variety of asset classes via stochastic optimal control theory. It is complete in the regard that calibration and estimation methodologies are provided and practical, numerical considerations are addressed, where appropriate. The major contributions to current academic literature are summarized below.

\section{- Market making in a single asset}

Chapter 3 addresses the problem of market making in a single asset in the presence of adverse selection and trade clustering. It is the first piece to explicitly derive an optimal market making strategy while accounting for asymmetric information in the market. The arrival of market orders is modeled as a bivariate Hawkes process, where the distinction is made between an MO being executed by an informed agent versus an uninformed one. Equipped with the tools presented in Chapter 5, the strategy is backtested on real data and yields positive results for each of the three considered stocks. The addition of a provision for the market maker to be aggressive and submit MOs when she detects trading from informed agents further illustrates the need to incorporate adverse selection in market microstructure models.

\section{- Algorithmic trading in multiple assets}

Chapter 4 develops a mathematical framework for an economy with multiple assets. Furthermore, these assets are permitted to have a structural dependence and bid/offer spreads that yields a non-trivial no-arbitrage region for their midprices. 
An optimal HFT strategy is derived for a profit maximizing agent that is inventory averse. We show that this strategy consists of two main components, a market making component and a statistical arbitrage component, that contribute to the strategy at complimentary times. This strategy appears to be the first to exploit the specific structure that is present in overcomplete markets. The added value to the investor is shown to be substantial when considering the over-completeness of the market versus treating the assets as simply correlated. Finally, numerical considerations are made and a dimensional reduction to the explicit numerical discretization scheme is also provided.

\section{- The Generalized Latent Hawkes process}

In response to the novel, yet seemingly complex, model presented in Chapter 3, two effecient, complimentary calibration methodologies are supplied. By utilizing Sequential Monte Carlo methods, a stepwise algorithm to construct on the fly estimators of the latent intensity process is also provided. The results developed in Chapter 5 provide the necessary bridge for the real-time application of the single asset market making strategy derived in Chapter 3. Furthermore, the generalization to allow for random, unobservable jumps is a feature that is a currently unexplored area in current literature and, as outlined in Chapter 5, has practical application in a variety of disciplines.

\section{2 $\quad$ Future Directions}

Although there are several directions upon which the research presented in this thesis can be further explored, the author would like to highlight some that he feels will be of the greatest impact on both the academic community and industry. Namely, extensions to other markets that do not operate through a centralized exchange with price-time priority, further developing the multiple asset framework described in Chapter 4, and making use of the Generalized Latent Hawkes model presented in Chapter 5 to better analyze and describe event data ${ }^{65}$. Each of these are summarized below.

\section{- Stylized adverse selection models}

The models for short-term alpha presented in Sections 3.4 and 4.4 .1 are just two ways to directly incorporate adverse selection into a microstructure model. Although these models offer qualititative features that are currently consistent with

\footnotetext{
${ }^{65}$ Although this thesis primarily focuses on high frequency trading and quantitative finance, the author acknowledges the vast application of Hawkes processes in other disciplines and encourages academics in these fields to explore.
} 
what is observed in real data, it is quite possible that a shift in agent behaviour will prompt the development of more stylized and involved models of adverse selection.

\section{- Exotic multiple asset frameworks}

Multiple asset economies in many asset classes have inherent structure different from being overcomplete, as was explored in Chapter 4. A prominent example is in foreign exchange (FX) where the product (as opposed to the linear combination in the overcomplete setting) of three traded assets is a constant. ${ }^{66}$ Whether it be in the context of optimal liquidation, hedging, or simply profit/utility maximization, HFT in economies with exotic constraints on their midprices continues to be an interesting and impactful area of research.

\section{- HFT with throttled activity}

In an active effort to thwart the ongoing technological arms race, some exchanges (e.g., EBS and Reuters) have begun to throttle activity. All activity sent to this exchange during the throttling window is amalgamated (according to some rules specific to each exchange) and then executed all at once at the end of the window. Typical lengths of of throttling windows are of the order of $100 \mathrm{~ms}$, but this varies according to the exchange.

As one might expect, this complicates the continuous time framework that most models are based on. In fact, even if one poses a model in event-space, where each discrete time step corresponds to a single market order submission or a limit order submission or cancellation, throttling will entail many trades occuring within the same event. The lack of event time granularity of individual event data, since all data is time stamped at the end of the throttling window, is also a major technical complication. This appears to a be a completely unexplored area of research that would be applicable in FX markets.

\section{- HFT without a centralized exchange}

Most microstructure models and HFT strategies are hinged on the idea of a single centralized exchange with a limit order book that all trades pass through, thus promoting transparancy in market activity. However, some asset classes (e.g., FX) do not trade on a single centralized exchange. ${ }^{67}$ Furthermore, a large portion of the traded volume can come from proprietary platforms supplied by large firms, thus making trade data inaccessible by anyone outside that firm.

Statistical complications arise when attempting to use trade data provided by an exchange as a proxy for the entire market. From a financial point of view, there is

\footnotetext{
${ }^{66}$ Consider the three traded currency pairs GBPUSD, EURUSD, and EURGBP as an example of such an economy where the relation EURUSD $=$ EURGBP $\times$ GBPUSD holds true up to the assets' bid/offer spreads.

${ }^{67}$ Although there are exchanges that amalgamate limit quotes and execute MOs in this way, issues such as latency and throttling cause complications when attempting to amalgamate them into one LOB.
} 
also a lack of transparency in what prices other market making firms are providing their clients through their own platforms making it difficult to determine another firm's fill probabilities when sending a particular set of quotes to a client. Acknowledging these market intricacies in a microstructure model and accounting for them in an HFT strategy has immediate industrial application and is ripe for academic development.

\section{- The Generalized Latent Hawkes model}

Chapter 5 outlines the various applications of the Hawkes process in seismology, neuroscience, genetics and molecular biology, and the social sciences. Section 5.6 details some other applications of the Generalized Latent Hawkes process within the context of quantitative finance. Although this thesis takes great strides to improve the practicality and efficiency of standard likelihood estimation methods within this class of models, it is believed that the derived methods can be further developed on both an efficiency and stability basis. 


\title{
Appendix A
}

\section{Acronyms and Notation}

\author{
A.1 Acronyms \\ AT Algorithmic trading \\ BBO Best bid and offer \\ bp Basis point \\ CDF Cumulative distribution function \\ DGP Data generating process \\ DPE Dynamic programming equation \\ DPP Dynamic programming principle \\ F-K Feynman-Kac \\ FP Fill probability \\ FX Foreign exchange \\ HF High frequency \\ HFT High frequency trading/trader \\ HJB Hamilton-Jacobi-Bellman \\ iid Independent and identically distributed \\ K-S Kolmogorov-Smirnov \\ LCRL Left continuous with right limits \\ LO Limit order \\ LOB Limit order book \\ LLH Log-likelihood
}




$\begin{array}{ll}\text { LRM } & \text { Long run mean } \\ \text { MCEM } & \text { Monte Carlo expectation maximization } \\ \text { MIR } & \text { Mean inventory risk } \\ \text { MLE } & \text { Maximum likelihood estimation/estimator } \\ \text { MM } & \text { Market maker } \\ \text { MO } & \text { Market order } \\ \text { ODE } & \text { Ordinary differential equation } \\ \text { P-P } & \text { Probability-probability } \\ \text { PDE } & \text { Partial differential equation } \\ \text { PIDE } & \text { Partial integro-differential equation } \\ \text { PIT } & \text { Probability integral transform } \\ \text { PnL } & \text { Profit and loss } \\ \text { QVI } & \text { Quasi-variational inequality } \\ \text { RCLL } & \text { Right continuous with left limits } \\ \text { SDE } & \text { Stochastic differential equation } \\ \text { SMC } & \text { Sequential Monte Carlo }\end{array}$




\section{A.2 Notation for Chapter 3}

\section{Stochastic Processes:}

$\alpha_{t} \quad$ Drift process of $S_{t}$

$B_{t} \quad$ Standard Brownian Motion

$\delta_{t}^{-}, \delta_{t}^{+} \quad$ Limit buy/sell order quote depth as measured from $S_{t}$

$\epsilon_{k}^{-}, \epsilon_{k}^{+}, \widetilde{\epsilon}_{k}^{-}, \widetilde{\epsilon}_{k}^{+} \quad$ iid sequences of exponential RV's

$\boldsymbol{\kappa}_{t}=\left(\kappa_{t}^{-}, \kappa_{t}^{+}\right) \quad$ Parameter describing the shape of the limit buy/sell side of the LOB

$\boldsymbol{\lambda}_{t}=\left(\lambda_{t}^{-}, \lambda_{t}^{+}\right) \quad$ Intensity of total market orders $\left(\bar{M}_{t}^{-}+\widetilde{M}_{t}^{-}, \bar{M}_{t}^{+}+\widetilde{M}_{t}^{+}\right)$

$\Lambda_{t}^{-}, \Lambda_{t}^{+} \quad$ Limit buy/sell fill rate facing the trader

$m_{t}^{ \pm}(u) \quad$ Mean of $\lambda_{u}^{ \pm}$conditional on $\mathcal{F}_{t}$

$\tilde{m}_{t}^{ \pm}(u) \quad$ Mean of $\kappa_{u}^{ \pm}$conditional on $\mathcal{F}_{t}$

$\bar{M}_{t}^{-}, \bar{M}_{t}^{+} \quad$ Counting process for influential market sell/buy orders

$\widetilde{M}_{t}^{-}, \widetilde{M}_{t}^{+} \quad$ Counting process for non-influential market sell/buy orders

$N_{t}^{-}, N_{t}^{+} \quad$ Counting process for filled limit buy/sell orders

$q_{t} \quad$ Inventory process

$S_{t} \quad$ Midprice process

$W_{t} \quad$ Standard Brownian Motion

$X_{t} \quad$ Cash process

$Z_{t}^{ \pm} \quad$ Poisson processes with intensity $\mu^{ \pm}$that cause jumps in $\boldsymbol{\lambda}_{t}$ and $\boldsymbol{\kappa}_{t}$

\section{Functions/Random Variables:}

$a, b ., c . \quad$ Functions in the exact expansions of $g$.

$B(\delta ; \boldsymbol{\kappa}) \quad$ Function that appears in the expansion of the optimal control

$\delta^{ \pm *} \quad$ Asymptotic optimal control

$\delta_{0}^{ \pm}, \delta^{ \pm} \quad$ Functions in the linear expansion of $\delta^{ \pm *}$

$\delta_{\text {opt }}^{ \pm *} \quad$ Exact optimal control

$\epsilon^{ \pm}, \widetilde{\epsilon}^{ \pm} \quad$ Shorthand for $\epsilon_{k}^{ \pm}, \widetilde{\epsilon}_{k}^{ \pm}$

$g \quad$ Part of the value function that is in excess of $x+q s$

$g_{0}, g$ Functions in the linear expansion of $g$

$h_{ \pm}(\delta ; \boldsymbol{\kappa}) \quad$ Fill probability function on the limit buy/sell side of the LOB

$\Phi \quad$ Value function 


\section{Constants:}

$\mathfrak{a}^{ \pm} \quad$ Scaled versions of $\mathbb{E}\left[\epsilon^{ \pm}\right]$with scale parameter $\varepsilon$

$\widetilde{\mathfrak{a}}^{ \pm} \quad$ Scaled versions of $\mathbb{E}\left[\widetilde{\epsilon}^{ \pm}\right]$with scale parameter $\varepsilon$

$\mathbf{A} \quad\left(\begin{array}{cc}\beta-\eta \rho & -\nu \rho \\ -\nu \rho & \beta-\eta \rho\end{array}\right)$

$\beta \quad$ Mean reversion rate of $\lambda_{t}^{ \pm}$

$\beta_{\kappa} \quad$ Mean reversion rate of $\kappa_{t}^{ \pm}$

$c_{\text {trans }} \quad$ Multiplicative liquidation transaction cost at time $T$

$d_{ \pm} \quad$ Shift parameter in the exponential and power FP functions

$\varepsilon \quad$ Multiplicative scale parameter of $\mathbb{E}\left[\epsilon^{ \pm}\right], \mathbb{E}\left[\widetilde{\epsilon}^{ \pm}\right]$

$\eta \quad$ Jump size of $\lambda_{t}^{ \pm}$due to arrival of $\bar{M}_{t}^{ \pm}$

$\tilde{\eta} \quad$ Jump size of $\lambda_{t}^{ \pm}$due to arrival of $Z_{t}^{ \pm}$

$\eta_{\kappa} \quad$ Jump size of $\kappa_{t}^{ \pm}$due to arrival of $\bar{M}_{t}^{ \pm}$

$\tilde{\eta}_{\kappa} \quad$ Jump size of $\kappa_{t}^{ \pm}$due to arrival of $Z_{t}^{ \pm}$

$\gamma^{ \pm} \quad$ Parameter in the power FP function

$\mu^{ \pm} \quad$ Intensity of $Z_{t}^{ \pm}$

$\nu \quad$ Jump size of $\lambda_{t}^{ \pm}$due to arrival of $\bar{M}_{t}^{\mp}$

$\tilde{\nu} \quad$ Jump size of $\lambda_{t}^{ \pm}$due to arrival of $Z_{t}^{\mp}$

$\nu_{\kappa} \quad$ Jump size of $\kappa_{t}^{ \pm}$due to arrival of $\bar{M}_{t}^{\mp}$

$\tilde{\nu}_{\kappa} \quad$ Jump size of $\kappa_{t}^{ \pm}$due to arrival of $Z_{t}^{\mp}$

$\phi \quad$ Inventory-aversion parameter

$\rho \quad$ Probability that any market order is influential

$\sigma \quad$ Volatility of $S_{t}$

$\sigma_{\alpha} \quad$ Volatility of $\alpha_{t}$

$\varsigma \quad \max \left(\phi,|\alpha|, \mathbb{E}\left[\epsilon^{ \pm}\right]\right)$

$\theta \quad$ Mean reversion level of $\lambda_{t}^{ \pm}$

$\theta_{\kappa} \quad$ Mean reversion level of $\kappa_{t}^{ \pm}$

$T \quad$ Trading time horizon (seconds)

$v \quad$ Constant term in the drift of $S_{t}$

$\zeta \quad$ Mean reversion rate of $\alpha_{t}$

$\boldsymbol{\zeta} \quad\left(\begin{array}{c}\beta \theta+\tilde{\eta} \mu^{-}+\tilde{\nu} \mu^{+} \\ \beta \theta+\tilde{\nu} \mu^{-}+\tilde{\eta} \mu^{+}\end{array}\right)$ 


\section{Other:}

$\begin{array}{ll}\mathcal{A} & \text { Set of all bounded } \mathcal{F}_{t} \text {-previsible functions } \\ C^{k} & \text { Set of } k \text {-times continuously differentiable functions } \\ \mathcal{F}_{t} & \text { Element of the natural filtration generated by all processes } \\ \overline{\mathcal{F}}_{t} & \text { Element of the natural filtration generated by }\left\{S_{t}, \epsilon_{M_{t}^{ \pm}}^{ \pm}, M_{t}^{ \pm}, N_{t}^{ \pm}\right\} \\ \mathbb{F} & \text { The filtration }\left\{\mathcal{F}_{t}\right\}_{0 \leq t \leq T} \\ \mathcal{L} & \text { The part of the generator of the processes } \alpha_{t}, \boldsymbol{\lambda}_{t}, \boldsymbol{\kappa}_{t}, \text { and } Z_{t}^{ \pm} \text {that does not } \\ & \text { depend on } \delta_{t}^{ \pm} \\ \Omega & \text { Sample space } \\ \mathbb{P} & \text { Probability measure on }\left(\Omega, \mathcal{F}_{T}\right) \text { equipped with filtration } \mathbb{F} \\ \mathbb{S}^{ \pm}, \tilde{\mathbb{S}}^{ \pm} & \text {Mean shift operators } \\ \mathcal{S}^{ \pm} & \text {Shift operators }\end{array}$




\section{A.3 Notation for Chapter 4}

\section{Stochastic Processes:}

$\boldsymbol{\alpha}_{t} \quad$ Drift process of $\mathbf{S}_{t}$

$\mathbf{B}_{t} \quad$ Standard Brownian Motion in $n$ dimensions

$\boldsymbol{\delta}_{t}=\left(\boldsymbol{\delta}_{t}^{-}, \boldsymbol{\delta}_{t}^{+}\right) \quad$ Vector of LO quote depths with components $\delta_{i, t}^{ \pm}$

$\mathfrak{d}_{(k)} \quad$ Sequence of random variables representing immediate market impact

$\mathfrak{e}_{(k)} \quad$ iid sequence of exponential random variables with mean 1

$\mathbf{M}_{t}^{-}, \mathbf{M}_{t}^{+} \quad$ Vector of counting processes for the investor's filled buy/sell LOs

$N_{t} \quad$ Total number of MOs from other market participants

$\bar{N}_{t} \quad$ Total number of MOs from all market participants

$\mathbf{N}_{t}^{-}, \mathbf{N}_{t}^{+} \quad$ Vectors of counting processes for MOs from other market participants with components $N_{i, t}^{ \pm}$

$\overline{\mathbf{N}}_{t}^{-}, \overline{\mathbf{N}}_{t}^{+} \quad$ Vectors of counting processes for MOs from all market participants with components $\bar{N}_{i, t}^{ \pm}$

$\widehat{\mathbf{N}}_{t}^{-}, \widehat{\mathbf{N}}_{t}^{+} \quad$ Vectors of counting processes for MOs from informed agents

$\mathbf{q}_{t} \quad$ Inventory process

$\mathbf{S}_{t} \quad$ Midprice process with component $S_{t}^{i}$

$\widehat{\mathbf{S}}_{t} \quad$ Unreflected version of $\mathbf{S}_{t}$

$\varsigma \quad$ Sequence represnting the investor's MO buy/sell indicator with element $\varsigma_{i}$

$\tau \quad$ Sequence of stopping times representing MO submissions with element $\tau_{i}$

$X_{t} \quad$ Cash process

\section{Functions/Random Variables:}

$g \quad$ Part of the value function that is in excess of $x+\mathbf{q} \cdot \mathbf{s}$

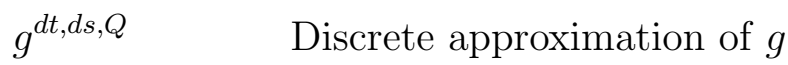

$\Gamma \quad$ Function related to dimensional reduction in spatial variable $\mathbf{s}$

$h_{i}^{-}(\delta), h_{i}^{+}(\delta) \quad$ Fill probability function on the limit buy/sell side of the LOB for asset $i$

$\ell(q) \quad$ Penalty function

$\Phi \quad$ Value function 


\section{Constants:}

$\mathbf{0} \quad\left(n-n^{\prime}\right)$-dimensional vector of 0's

b Element of $\mathbb{R}^{n}$ used in the condition in definition of over-complete market

$e_{i} \quad$ Vector with 1 in the $i^{\text {th }}$ position and 0 elsewhere

$\epsilon \quad$ Scale factor for $\Psi$

$\gamma \quad$ Terminal liquidation market impact parameter

$\kappa_{i}^{-}, \kappa_{i}^{+} \quad$ Scale parameters in exponential fill rate functions

$\lambda_{i}^{-}, \lambda_{i}^{+} \quad$ Intensity of market orders counting processes $N_{t}^{-}, N_{t}^{+}$

$n \quad$ Number of risky assets

$n^{\prime} \quad$ Dimensionality after reduction

$\boldsymbol{n} \quad$ Normal vector

$\phi \quad$ Inventory penalty parameter

$\Psi \quad$ Mean jump size matrix for $\alpha_{t}$

$Q \quad$ Upper limit on inventory for each asset

$\rho \quad$ Probability an MO is influential

$\boldsymbol{\sigma} \quad$ Volatility matrix

$T \quad$ Trading horizon

$\bar{T} \quad$ Investment horizon in the condition in definition of over-complete market

$T_{1}, T_{2} \quad$ Maturity of first and second nearby futures contracts in examples

$\boldsymbol{\xi} \quad$ Vector of spreads with component $\xi_{i}$

$\boldsymbol{\zeta} \quad$ Mean reversion rate matrix for $\boldsymbol{\alpha}_{t}$

\section{Other:}
$\mathcal{A} \quad$ No-arbitrage region
$\partial \mathcal{A} \quad$ Boundary of the set $\mathcal{A}$
$\overline{\mathcal{A}} \quad$ Closure of the set $\mathcal{A}$
$\mathcal{A}^{\prime} \quad$ Dimensionally reduced no-arbitrage region
$\overline{\mathcal{A}}^{\prime} \quad$ Closure of $\mathcal{A}^{\prime}$
$B \quad$ Subspace containing all vectors $\mathbf{b}$ from definition of over-complete market
$B^{\perp} \quad$ Orthogonal compliment of $B$
$C^{k} \quad$ Set of $k$-times continuously differentiable functions
$C_{b}^{k} \quad$ Set of $k$-times continuously differentiable functions with bounded derivatives
$\Delta_{i}^{ \pm} \quad$ Difference operator associated with a filled LO 
$\mathcal{F}_{t} \quad$ Element of the natural filtration generated by $\left(\mathbf{S}_{t}, \mathbf{M}_{t}^{ \pm}, \mathbf{N}_{t}^{ \pm}, \overline{\mathbf{N}}_{t}^{ \pm}\right)$

$\mathbb{F} \quad$ The filtration $\left\{\mathcal{F}_{t}\right\}_{0 \leq t \leq T}$

$L^{2} \quad$ Set of all square-integrable functions on $\left(\Omega, \mathcal{F}_{\bar{T}}, \mathbb{P}\right)$

$\mathcal{L} \quad$ Infinitesimal generator of $\mathbf{S}_{t}$ under Assumption 4.2.4

$\mathcal{M}^{d t, d s, Q}$ Discrete dynamic evolution operator for the impulse region

$\Omega \quad$ Sample space

$\mathbb{P} \quad$ Probability measure on $\left(\Omega, \mathcal{F}_{\bar{T}}\right)$ equipped with filtration $\mathbb{F}$

$\mathbb{S}_{d s} \quad n$-dimensional regular partition of the dimensionally reduced $\mathbf{s}$-grid

$\mathcal{S}^{d t, d s, Q} \quad$ Discrete combined dynamic evolution operator

$\mathbb{T}_{d t} \quad$ Regular time partition

$\mathcal{T}^{d t, d s, Q} \quad$ Discrete dynamic evolution operator for the continuation region

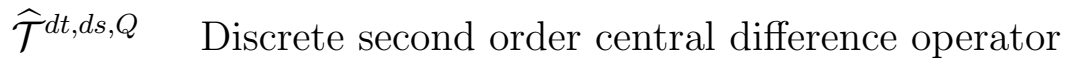

$\mathcal{X} \quad$ Set of all admissible strategies

$\mathbb{Y}_{Q} \quad$ Subset of $\mathbb{Z}^{n}$ that corresponds to inventory with maximum $Q$

$\nabla \quad$ Gradient operator in the s component

$\langle\cdot\rangle_{t} \quad$ Quadratic variation operator 


\section{A.4 Notation for Chapter 5}

\section{Stochastic Processes:}

$\boldsymbol{\eta}_{k} \quad$ iid sequence of random matrices

$\boldsymbol{\eta}_{t} \quad$ Shorthand for $\boldsymbol{\eta}_{\sum N_{i, t-}}$

$\boldsymbol{\lambda}_{t} \quad$ Intensity process of $\mathbf{N}_{t}$

$\mathbf{N}_{t} \quad$ Vector of counting processes with $i^{\text {th }}$ component $N_{i, t}$

\section{Functions/Random Variables:}

$\begin{array}{ll}\eta_{i j} & (i, j) \text { element of } \boldsymbol{\eta} \\ \boldsymbol{\eta} & \text { Random matrix with the same distribution as any element of } \boldsymbol{\eta}_{1} \\ f(x \mid y) & \mathbb{P}\left[\boldsymbol{\lambda}_{t_{k}} \in d x \mid \boldsymbol{\lambda}_{t_{k-1}}=y\right] \\ g(t, l \mid y) & \mathbb{P}\left[T_{k}(1) \in d t, T_{k}(2)=l \mid \boldsymbol{\lambda}_{t_{k-1}}=y\right] \\ l\left(q \mid y, S_{1}, S_{2}\right) & \mathbb{P}\left[\boldsymbol{\eta}_{t_{k}}=\boldsymbol{\eta}_{S_{1}(1)}(q) \mid \boldsymbol{\lambda}_{t_{k-1}}=y, T_{k}=S_{1}, T_{k+1}=S_{2}\right] \\ \mathcal{L} & \text { Likelihood function } \\ \overline{\mathcal{L}} & \text { Pseudo-likelihood function used in Algorithm I } \\ \tilde{\mathcal{L}} & \text { Isolated likelihood function used in Algorithm I } \\ \mathcal{L} & \text { Composite marginal likelihood function } \\ m(N) ; y) & \text { Size of the largest cluster in a data set of size } N \\ \boldsymbol{\nu} & \boldsymbol{\rho}=1 \text { MLE of } \boldsymbol{\eta} \\ Q(\xi \mid Y) & \text { Maximizer of } \overline{\mathcal{L}} \\ \boldsymbol{\rho}_{k}, \boldsymbol{\beta}_{k}, \boldsymbol{\theta}_{k}, \boldsymbol{\eta}_{k} & \text { Parameter estimates after } k \text { iterations of Algorithm I } \\ \rho_{\text {div }}, \beta_{i, \text { div }} & \text { Divergence difference of } \boldsymbol{\rho}, \beta_{i} \\ t_{i} & \text { Time of the } i^{\text {th }} \text { event with component label } \\ T_{i} & \text { Inter-arrival time of the } i\end{array}$

\section{Constants:}

$0 \quad n \times n$ matrix of zeros

$\mathbf{1}_{n} \quad n$-dimensional vector of 1's

$\delta_{i j} \quad$ Suggested minimum difference in the possible values of $\eta_{i j}$

$e_{i} \quad n$-dimensional unit vector with 1 in the $i^{\text {th }}$ component 


$\begin{array}{ll}\epsilon & \text { Tolerance used in renewal point identification } \\ \boldsymbol{\beta} & \text { Mean reversion rate matrix of } \boldsymbol{\lambda}_{t} \\ \boldsymbol{\eta}(k), \eta_{i j}(k) & k^{\text {th }} \text { possible value of } \boldsymbol{\eta}, \eta_{i j} \\ \mathbb{I}_{n} & n \text {-dimensional identity matrix } \\ m & \text { Cluster size in Algorithm I } \\ M & \text { Number of particles in SMC particle filter } \\ n & \text { Dimension of } \mathbf{N}_{t} \\ N & \text { Total number of points } \\ p & \text { Size of the support of } \boldsymbol{\eta} \\ \rho_{i} & (i+1)^{\text {th }} \text { element of } \boldsymbol{\rho} \\ \rho & \text { Shorthand for } \rho_{1} \text { when } p=2 \\ \boldsymbol{\rho} & \text { Vector of probability weights on the distribution of } \boldsymbol{\eta} \\ \theta_{i}, \beta_{i} & i^{\text {th }} \text { element of } \boldsymbol{\theta}, \boldsymbol{\beta} \text { (when } \boldsymbol{\beta} \text { is diagonal) } \\ \boldsymbol{\theta} & \text { Mean reversion level of } \boldsymbol{\lambda}_{t} \\ T & \text { Upper time limit of sample period } \\ \Theta & \text { Element of the parameter space }\end{array}$

\section{Other:}

$\begin{array}{ll}C_{k}^{m} & \text { A cluster of } m+1 \text { points beginning with } t_{k} \\ \mathcal{F}_{t} & \text { Element of the natural filtration generated by }\left\{\mathbf{N}_{t}\right\},\left\{\boldsymbol{\eta}_{t}\right\} \\ \overline{\mathcal{F}}_{t} & \text { Element of the natural filtration generated by }\left\{\mathbf{N}_{t}\right\} \\ \Omega & \text { Sample space } \\ \mathbb{P} & \text { Probability measure on }\left(\Omega, \mathcal{F}_{T}\right)\end{array}$




\section{Appendix B}

\section{Supplementary Background Material}

This Appendix provides some supplementary material related to results that are used throughout the body of this thesis.

\section{B.1 Hamilton-Jacobi-Bellman Equations}

This section states the Hamilton-Jacobi-Bellman equations for a stochastic control problem and a combined stochastic control and impulse control problem. For detailed derivations of these equations, please refer to Øksendal and Sulem (2007).

Define $\mathcal{S} \subseteq \mathbb{R}^{k}$ and let $\left\{Y_{t}\right\}_{t \geq 0}$ be a ("well-defined") jump diffusion where the drift, volatility, and jump measure can be controlled by some other process $u_{t}$ that is assumed to be RCLL and adapted to $\mathcal{F}_{t}$. Hence, $Y_{t}$ (or more specifically, $Y_{t}^{(u)}$ ) is a controlled jump diffusion.

The performance criterion is given by

$$
J^{(u)}(t, y)=\mathbb{E}_{(t, y)}\left[\int_{t}^{\tau} f\left(Y_{s}, u_{s}\right) d s+g\left(Y_{\tau}\right) \mathbf{1}_{\{\tau<\infty\}}\right]
$$

where $\tau=\inf \left\{t \geq 0: Y_{t}^{(u)} \notin \mathcal{S}\right\}$ and $\mathbb{E}_{(t, y)}[\cdot]$ is shorthand for $\mathbb{E}\left[\cdot \mid Y_{t}=y\right]$.

Define the value function by

$$
\Phi(t, y)=\sup _{u \in \mathcal{U}} J^{(u)}(t, y)
$$


where $\mathcal{U}$ is the set of admissible controls.

For any (admissible) Markov control $u_{t}$, denote the generator of $Y_{t}$ by $\mathcal{L}^{(u)}$.

Through a dynamic programming argument analogous to that of Section 2.2, the HJB equation for the value function in (B.1) is:

$$
\left\{\begin{aligned}
\partial_{t} \Phi+\sup _{u}\left\{f(y, u)+\mathcal{L}^{(u)} \Phi\right\} & =0 \quad y \in \mathcal{S} \\
\Phi(t, y) & =g \quad y \notin \mathcal{S}
\end{aligned}\right.
$$

Now suppose that we are able to intervene with impulse controls. More precisely, define the impulse control as the following double sequence:

$$
v=\left(\tau_{1}, \tau_{2}, \ldots ; \varsigma_{1}, \varsigma_{2}, \ldots\right)
$$

where $0 \leq \tau_{1} \leq \tau_{2} \leq \ldots$ are $\mathcal{F}_{t}$ stopping times, and $\mathcal{F}_{\tau_{j}}$ measurable impulses $\varsigma_{j}(j \in \mathbb{N})$. For a given $v$, the impulse control affects the controlled process $Y_{t}$ at the impulse times via

$$
Y_{\tau_{j}}^{(u, v)}=\Gamma\left(Y_{\tau_{j}-}+\Delta_{N} Y_{\tau_{j}}, \varsigma_{j}\right)
$$

for some function $\Gamma$, where $\Delta_{N} Y_{t}$ is the jump in $Y_{t}$ due to a jump in the jump diffusion component of $Y_{t}$.

One may also impose a cost function associated with such impulses. This is done by adding an extra term in the performance criterion:

$$
\tilde{J}^{(u, v)}(t, y)=\mathbb{E}_{(t, y)}\left[\int_{t}^{\tau} f\left(Y_{s}, u_{s}\right) d s+g\left(Y_{\tau}\right) \mathbf{1}_{\{\tau<\infty\}}+\sum_{\tau_{j} \leq \tau} K\left(Y_{\tau_{j}-}+\Delta_{N} Y_{\tau_{j}}, \varsigma_{j}\right)\right] .
$$

Now define the value function as

$$
\tilde{\Phi}(t, y)=\sup _{(u, v)} \tilde{J}^{(u, v)}(t, y) .
$$

Through a dynamic programming argument analogous to that of Section 2.4, the HJB 
quasi-variational inequality (QVI) for the value function in (B.3) is then

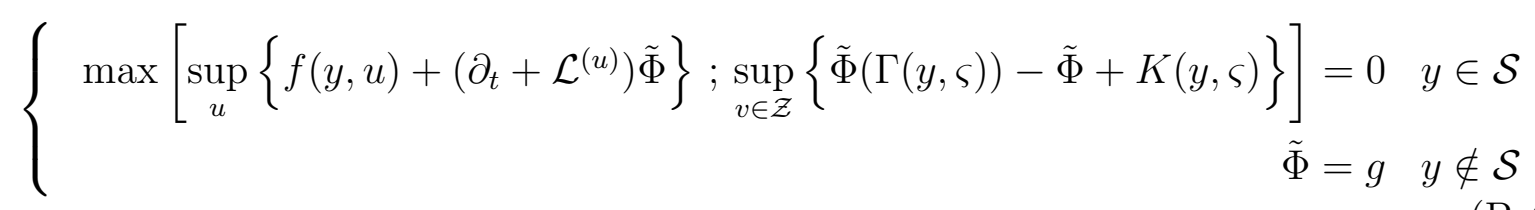

where $\mathcal{Z}$ is the set of admissible impulse controls.

\section{B.2 Viscosity Solutions}

The results in Appendix B.1 are extremely convenient when the value function $\Phi$ is suffieciently smooth on $\mathcal{S}$ (i.e. $\Phi$ is $C^{2}$ on the interior of $\mathcal{S}$ and $C^{1}$ at the boundary). However, this need not be the case. In such scenarios, the expression $\mathcal{L} \Phi$ does not make sense. To this end, we define the notion of viscosity solutions.

Definition B.2.1. Let $\Phi \in C(\overline{\mathcal{S}})$.

(i) We say that $\Phi$ is a viscosity subsolution of (B.4) if for any $t_{0} \geq 0, x_{0} \in \operatorname{int}(\mathcal{S})$, and $h(t, x) \in C^{1}\left(\mathbb{R}_{+}\right) \cap C^{2}\left(\mathbb{R}^{k}\right)$ such that $h\left(t_{0}, x_{0}\right)=\Phi\left(t_{0}, x_{0}\right)$ and $h(t, x) \leq \Phi(t, x)$ on $\mathcal{S}$, we then have $h(t, x)$ satisfying (B.4).

(ii) We say that $\Phi$ is a viscosity supersolution of (B.4) if for any $t_{0} \geq 0, x_{0} \in \operatorname{int}(\mathcal{S})$, and $h(t, x) \in C^{1}\left(\mathbb{R}_{+}\right) \cap C^{2}\left(\mathbb{R}^{k}\right)$ such that $h\left(t_{0}, x_{0}\right)=\Phi\left(t_{0}, x_{0}\right)$ and $h(t, x) \geq \Phi(t, x)$ on $\mathcal{S}$, we then have $h(t, x)$ satisfying (B.4).

(iii) We say that $\Phi$ is a viscosity solution of (B.4) if it is both a viscosity subsolution and supersolution.

\section{B.3 Feynman-Kac Forumla for Jump Diffusions}

Let $\left\{X_{t}\right\}_{0 \leq t \leq T}$ be a jump diffusion on $\mathbb{R}^{k}$ with $\mathbb{P}$-generator $\mathcal{L}$. Suppose that some function $u(t, x)$ satisfies the following partial integro-differential equation (PIDE) with boundary condition:

$$
\left\{\begin{aligned}
\left(\partial_{t}+\mathcal{L}\right) u(t, x)+f(t, x) & =V(t, x) u(t, x) \\
u(x, T) & =\varphi(x)
\end{aligned}\right.
$$


Lemma B.3.1. Feynman-Kac Formula. The solution to (B.5) has the following stochastic representation:

$$
u(t, x)=\mathbb{E}^{\mathbb{P}}\left[\int_{t}^{T} e^{-\int_{t}^{s} V\left(z, X_{z}\right) d z} f\left(s, X_{s}\right) d s+e^{-\int_{t}^{T} V\left(z, X_{z}\right) d z} \varphi\left(X_{T}\right) \mid X_{t}=x\right]
$$

Proof. Suppose $u(t, x)$ is a solution to (B.5) and consider the process

$$
Y\left(r, X_{r}\right):=\int_{t}^{r} e^{-\int_{t}^{s} V\left(z, X_{z}\right) d z} f\left(s, X_{s}\right) d s+e^{-\int_{t}^{r} V\left(z, X_{z}\right) d z} u\left(r, X_{r}\right) .
$$

It then follows that

$$
\mathbb{E}_{t, x}\left[Y\left(T, X_{T}\right)-Y\left(t, X_{t}\right)\right]=\mathbb{E}_{t, x}\left[\int_{t}^{T} e^{-\int_{t}^{r} V}\left\{f+\left(\partial_{r}+\mathcal{L}\right) u-V u\right\} d r\right]=0 .
$$

The first equality is true by Dynkin's Formula. The second equality is due to the condition in (B.5). Hence, we have

$$
\begin{aligned}
u(t, x) & =Y(t, x)=\mathbb{E}_{t, x}\left[Y\left(t, X_{t}\right)\right]=\mathbb{E}_{t, x}\left[Y\left(T, X_{T}\right)\right] \\
& =\mathbb{E}_{t, x}\left[\int_{t}^{T} e^{-\int_{t}^{s} V\left(z, X_{z}\right) d z} f\left(s, X_{s}\right) d s+e^{-\int_{t}^{T} V\left(z, X_{z}\right) d z} u\left(T, X_{T}\right)\right] \\
& =\mathbb{E}_{t, x}\left[\int_{t}^{T} e^{-\int_{t}^{s} V\left(z, X_{z}\right) d z} f\left(s, X_{s}\right) d s+e^{-\int_{t}^{T} V\left(z, X_{z}\right) d z} \varphi\left(X_{T}\right)\right] .
\end{aligned}
$$

It is interesting to note that Lemma B.3.1 is a special case of the result presented in Equation (B.2) of Appendix B.1 where the set of admissible controls $\mathcal{U}$ contains exactly one element. 


\section{Appendix $\mathrm{C}$}

\section{Proofs}

\section{C.1 Proof of Lemma 3.2.2}

Integrating both sides of (3.2), taking conditional expectation, applying Fubini's Theorem, and then taking derivative gives the following coupled system of ODEs for $m_{t}^{ \pm}(u)$ :

$$
\frac{d}{d u}\left(\begin{array}{l}
m_{t}^{-}(u) \\
m_{t}^{+}(u)
\end{array}\right)+\left(\begin{array}{cc}
\beta-\eta \rho & -\nu \rho \\
-\nu \rho & \beta-\eta \rho
\end{array}\right)\left(\begin{array}{l}
m_{t}^{-}(u) \\
m_{t}^{+}(u)
\end{array}\right)-\left(\begin{array}{c}
\beta \theta+\tilde{\eta} \mu^{-}+\tilde{\nu} \mu^{+} \\
\beta \theta+\tilde{\nu} \mu^{-}+\tilde{\eta} \mu^{+}
\end{array}\right)=\left(\begin{array}{l}
0 \\
0
\end{array}\right)
$$

with initial conditions $m_{t}^{ \pm}(t)=\lambda_{t}^{ \pm}$. This is a standard matrix equation, and, if $\mathbf{A}$ is invertible, it admits the unique solution

$$
\left(\begin{array}{l}
m_{t}^{-}(u) \\
m_{t}^{+}(u)
\end{array}\right)=e^{-\mathbf{A}(u-t)}\left[\left(\begin{array}{c}
\lambda_{t}^{-} \\
\lambda_{t}^{+}
\end{array}\right)-\mathbf{A}^{-1} \zeta\right]+\mathbf{A}^{-1} \zeta
$$

Since $\mathbf{A}$ is symmetric, it is diagonalizable by an orthonormal matrix $\mathbf{U}$. Furthermore, its eigenvalues are $\beta-(\eta \pm \nu) \rho$. Clearly, the limit $u \rightarrow \infty, m_{t}(u)$ converges if and only if $\beta-(\eta \pm \nu) \rho>0$, which implies $\beta>(\eta+\nu) \rho$ since $\eta, \nu, \rho \geq 0$.

The remaining case is if $\mathbf{A}$ has is not invertivle (i.e. has at least one zero eigenvalue). However, it is easy to see that in this case, the solution to (C.1) has at least one of $m_{t}^{ \pm}(u)$ growing linearly as a function of $u$. Furthermore, if at least one eigenvalue is zero, then either $\beta=(\eta-\nu) \rho$ or $\beta=(\eta+\nu) \rho$, which lie outside the stated bounds. 


\section{C.2 Proof of Proposition 3.5.1}

Applying the ansatz of the form on $\Phi$, differentiating inside the supremum in Equation (3.7) with respect to $\delta^{ \pm}$, expanding $g$ using the specified ansatz, writing $\delta^{ \pm *}=\delta_{0}^{ \pm}+\alpha \delta_{\alpha}^{ \pm}+$ $\varepsilon \delta_{\varepsilon}^{ \pm}+\phi \delta_{\phi}^{ \pm}+o(\varsigma)$, and setting the resulting equation to 0 gives our first-order optimality condition. To this order, the first-order conditions imply that

$$
\begin{aligned}
& h_{ \pm}\left(\delta_{0}^{ \pm}\right)+\delta_{0}^{ \pm} h_{ \pm}^{\prime}\left(\delta_{0}^{ \pm}\right)+\alpha\left\{\delta_{\alpha}\left(h_{ \pm}^{\prime \prime}\left(\delta_{0}^{ \pm}\right)+2 h_{ \pm}^{\prime}\left(\delta_{0}^{ \pm}\right)\right)+h^{\prime}\left(\delta_{0}^{ \pm}\right)\left(\mathbb{S}_{q \lambda}^{ \pm} g_{\alpha}-\mathbb{S}_{\lambda}^{ \pm} g_{\alpha}\right)\right\} \\
&+\varepsilon\left\{\delta_{\varepsilon}\left(h_{ \pm}^{\prime \prime}\left(\delta_{0}^{ \pm}\right)+2 h_{ \pm}^{\prime}\left(\delta_{0}^{ \pm}\right)\right)+h_{ \pm}^{\prime}\left(\delta_{0}^{ \pm}\right)\left(\mathbb{S}_{q \lambda}^{ \pm} g_{\varepsilon}-\mathbb{S}_{\lambda}^{ \pm} g_{\varepsilon}+ \pm \rho \mathfrak{a}^{ \pm} \mathcal{S}_{q \lambda}^{ \pm} g_{\alpha}\right)\right\} \\
&+\phi\left\{\delta_{\phi}\left(h_{ \pm}^{\prime \prime}\left(\delta_{0}^{ \pm}\right)+2 h_{ \pm}^{\prime}\left(\delta_{0}^{ \pm}\right)\right)+h_{ \pm}^{\prime}\left(\delta_{0}^{ \pm}\right)\left(\mathbb{S}_{q \lambda}^{ \pm} g_{\phi}-\mathbb{S}_{\lambda}^{ \pm} g_{\phi}\right)\right\}=o(\varsigma) .
\end{aligned}
$$

Observe that the Taylor expansion of $h(\delta)$ about $\delta_{0}$ requires the $C^{2}$ regularity condition to keep the error of the correct order. The $C^{1}$ regularity condition ensures that the global maximizer satisfies (C.3). Setting the constant term in (C.3) to zero yields (3.13). Setting the coefficients of $\alpha, \varepsilon$, and $\phi$ each separately to zero and solving for $\delta_{\alpha}, \delta_{\varepsilon}$, and $\delta_{\phi}$ results in (3.11). The finiteness of the optimal control correct to this order is ensured by the last condition in Assumption 3.3.1.

The existence of a solution to (3.13) is clear by noticing that the solution is a critical point of the continuous function $\delta h(\delta)$. The critical point exists since $\delta h(\delta)$ is non-positive for $\delta \leq 0$, is strictly positive on an open interval of the form $(0, d)$ due to $h \in C^{1}$ (since $h>0$ in an open neighbourhood of $\delta=0$ ), and goes to 0 in the limit (when $\delta \rightarrow \infty$ ) by Assumption 3.3.1.

To see that the exact values of the optimal controls are non-negative, observe that the value function is increasing in $x$. Therefore, $\Phi(t, x+\delta, \cdot)<\Phi(t, x, \cdot)$ for any $\delta<0$. Since the shift operators appearing in the argument of the supremum are linear operators, and $h(\delta ; \kappa)$ is bounded above by 1 and attains this maxima at $\delta=0$, the $\delta=0$ strategy dominates all strategies which have $\delta<0$.

\section{C.3 Proof of Theorem 3.5.2}

Inserting the expansion for $g$ and the feedback controls (3.11) for $\delta$ into the HJB equation in (3.7), and carrying out tedious but ultimately straightforward expansions, to order $\varsigma$, 
Equation (3.7) reduces to

$$
\begin{aligned}
o(\varsigma)=\mathcal{D} g_{0}+\left(\lambda^{+} \delta_{0}^{+} h_{+}\left(\delta_{0}^{+}\right)+\lambda^{-} \delta_{0}^{-} h_{-}\left(\delta_{0}^{-}\right)\right) \\
+\alpha\left\{q+(\mathcal{D}-\zeta) g_{\alpha}+\lambda^{+} h_{+}\left(\delta_{0}\right)\left[\mathbb{S}_{q \lambda}^{+}-\mathbb{S}_{\lambda}^{+}\right] g_{\alpha}+\lambda^{-} h_{-}\left(\delta_{0}\right)\left[\mathbb{S}_{q \lambda}^{-}-\mathbb{S}_{\lambda}^{-}\right] g_{\alpha}\right\} \\
+\varepsilon\left\{\mathcal{D} g_{\varepsilon}+\lambda^{+} h_{+}\left(\delta_{0}\right)\left(\left[\mathbb{S}_{q \lambda}^{+}-\mathbb{S}_{\lambda}^{+}\right] g_{\varepsilon}+\rho \mathfrak{a}^{+} \mathcal{S}_{q \lambda}^{+} g_{\alpha}\right)\right. \\
\left.\quad+\lambda^{-} h_{-}\left(\delta_{0}\right)\left(\left[\mathbb{S}_{q \lambda}^{-}-\mathbb{S}_{\lambda}^{-}\right] g_{\varepsilon}-\rho \mathfrak{a}^{-} \mathcal{S}_{q \lambda}^{-} g_{\alpha}\right)\right\} \\
+\phi\left\{-q^{2}+\mathcal{D} g_{\phi}+\lambda^{+} h_{+}\left(\delta_{0}\right)\left[\mathbb{S}_{q \lambda}^{+}-\mathbb{S}_{\lambda}^{+}\right] g_{\phi}+\lambda^{-} h_{-}\left(\delta_{0}\right)\left[\mathbb{S}_{q \lambda}^{-}-\mathbb{S}_{\lambda}^{-}\right] g_{\phi}\right\}
\end{aligned}
$$

where $\mathcal{D}=\partial_{t}+\mathcal{L}$ and the boundary conditions $g_{0}(T, \cdot)=g_{\alpha}(T, \cdot)=g_{\varepsilon}(T, \cdot)=g_{\phi}(T, \cdot)=0$ apply. Clearly, $g_{0}$ is independent of $q$ and, as seen in Proposition 3.5.1, does not affect the optimal strategy. Next, perform the following steps: (i) set the coefficients of $\alpha, \varepsilon$, and $\phi$ to zero separately; (ii) write $g_{\alpha}, g_{\varepsilon}$, and $g_{\phi}$ as in (3.14); and (iii) collect powers of $q$, and set them individually to zero. ${ }^{68}$ Then one finds the following equations for the functions $b_{\alpha}(t), b_{\varepsilon}(t, \boldsymbol{\lambda}), b_{\phi}(t, \boldsymbol{\lambda}, \boldsymbol{\kappa})$, and $c_{\phi}(t)$ :

$$
\begin{array}{rlr}
0 & =\mathcal{D} b_{\alpha}-\zeta b_{\alpha}+\lambda^{+}\left[\mathbb{S}_{\lambda}^{+}-1\right] b_{\alpha}+\lambda^{-}\left[\mathbb{S}_{\lambda}^{-}-1\right] b_{\alpha}+1, & \text { (C.5a) } \\
0 & =\mathcal{D} b_{\varepsilon}+\lambda^{+}\left[\mathbb{S}_{\lambda}^{+}-1\right] b_{\varepsilon}+\lambda^{-}\left[\mathbb{S}_{\lambda}^{-}-1\right] b_{\varepsilon}+\left\{\rho\left(\lambda^{+} \mathfrak{a}^{+}-\lambda^{-} \mathfrak{a}^{-}\right)+\left(\mu^{+} \tilde{\mathfrak{a}}^{+}-\mu^{-} \tilde{\mathfrak{a}}^{-}\right)\right\} b_{\alpha}, \\
& & \text { (C.5b) } \\
0 & =\mathcal{D} b_{\phi}+\lambda^{+}\left[\mathbb{S}_{\lambda}^{+}-1\right] b_{\phi}+\lambda^{-}\left[\mathbb{S}_{\lambda}^{-}-1\right] b_{\phi}-2 h\left(\delta_{0}\right)\left(\lambda^{+}-\lambda^{-}\right) c_{\phi}, & \text { (C.5c) } \\
0 & =\mathcal{D} c_{\phi}+\lambda^{+}\left[\mathbb{S}_{\lambda}^{+}-1\right] c_{\phi}+\lambda^{-}\left[\mathbb{S}_{\lambda}^{-}-1\right] c_{\phi}-1 . & \text { (C.5d) }
\end{array}
$$

These equations, together with the boundary conditions $b_{\alpha}(T, \cdot)=b_{\varepsilon}(T, \cdot)=b_{\phi}(T, \cdot)=$ $c_{\phi}(T, \cdot)=0$, admit, through a Feynman-Kac argument, the solutions presented in (3.15). More specifically, we apply the Feynman-Kac formula in Lemma B.3.1 to link the solution of the derived PIDE back to its stochastic representation, as presented in (3.15).

The functions $a_{\alpha}, a_{\varepsilon}$ and $a_{\phi}$ are independent of $q$ and, since the optimal spreads given in (3.12) contain difference operators in $q$ which vanish when they act on functions independent of $q$, do not influence the optimal strategy.

\section{C.4 Proof of Corollary 3.5.3}

By applying Equation (3.14) for $g_{\alpha}, g_{\varepsilon}$ and $g_{\phi}$ in Theorem 3.5.2 to Equations (3.11) and (3.12) of Proposition 3.5.1 and using the fact that the $a, b$ and $c$ functions are all

\footnotetext{
${ }^{68}$ Note that this step is not an asymptotic expansion in $q$. Rather, it is exact given the prescribed expansion in the other parameters.
} 
independent of $q, \delta^{* \pm}$ reduces, after some tedious computations, to

$$
\delta^{ \pm *}=\delta_{0}^{ \pm}+B\left(\delta_{0}^{ \pm} ; \boldsymbol{\kappa}^{ \pm}\right)\left\{ \pm \alpha b_{\alpha}+\varepsilon\left( \pm \mathbb{S}_{\lambda}^{ \pm} b_{\varepsilon}+\rho \mathfrak{a}^{ \pm} b_{\alpha}\right)+\phi\left( \pm \mathbb{S}_{\lambda}^{ \pm} b_{\phi}+(1 \mp 2 q)(T-t)\right)\right\}
$$

Next, observing that $\pm \alpha b_{\alpha}+\varepsilon\left( \pm \mathbb{S}_{\lambda}^{ \pm} b_{\varepsilon}+\rho \mathfrak{a}^{ \pm} b_{\alpha}\right)= \pm \mathbb{S}_{\lambda}^{ \pm}\left(\mathbb{E}\left[\int_{t}^{T} \alpha_{u} d u\right]\right)$ gives (3.16). Finally, let $\delta_{\mathrm{opt}}^{ \pm}$denote the exact optimal controls. Using Proposition 3.5.1, we obtain that $\delta_{\mathrm{opt}}^{ \pm}$is non-negative and $\left|\delta^{*}-\delta_{\mathrm{opt}}^{ \pm}\right|=o(\varsigma)$. Therefore,

$$
\left|\max \left\{\delta^{ \pm *}, 0\right\}-\delta_{\mathrm{opt}}^{ \pm}\right| \leq\left|\delta^{ \pm *}-\delta_{\mathrm{opt}}^{ \pm}\right|=o(\varsigma)
$$

completes the proof.

\section{C.5 Proof of Lemma 4.5.1:}

Define $m:=\operatorname{dim} B$. Then $B=\operatorname{span}\left\{b_{1}, \ldots, b_{m}\right\}$, where $b_{1}, \ldots, b_{m}$ is an orthogonal basis, and for each $i=1, \ldots, m$ we have $($ wlog $) \mathbb{P}\left[b_{i} \cdot \mathbf{S}_{\bar{T}}=0\right]=1$. No arbitrage (i.e. $\mathbf{s} \in \mathcal{A}$ ) is equivalent to the system of inequalities

$$
y \cdot \mathbf{s}+\sum_{j=1}^{n}\left|y_{j}\right| \frac{\xi_{j}}{2}>0 \quad \text { for } y= \pm b_{1}, \ldots, \pm b_{m}
$$

which simplifies to

$$
-\sum_{j=1}^{n}\left|y_{j}\right| \frac{\xi_{j}}{2}<y \cdot \mathbf{s}<\sum_{j=1}^{n}\left|y_{j}\right| \frac{\xi_{j}}{2} \quad \text { for } y=b_{1}, \ldots, b_{m}
$$

Because the $b_{i}$ 's are linearly independent, one can see that (C.6) is equivalent to $\Gamma(\mathbf{s})$ being inside some rectangular subset of $\mathbb{R}^{m}$ for the function $\Gamma(\mathbf{s})=\left[b_{j} \cdot \mathbf{s}\right]_{j}$, which is also clearly $C^{2}$. At this point, $(I I)$ should be obvious from (C.6) and the definition of $\Gamma$, $(I I I)$ is proven, and linear independence of the basis vectors gives $(I V)$.

To prove $(I)$, we must appeal to some invariance properties of $g$. Notice that $\overline{\mathcal{A}}$ can now be reparametrized to the product space $\prod_{i=1}^{m}\left[-\sum_{j=1}^{n}\left|b_{i} \cdot e_{j}\right| \frac{\xi_{j}}{2}, \sum_{j=1}^{n}\left|b_{i} \cdot e_{j}\right| \frac{\xi_{j}}{2}\right] \times \mathbb{R}^{n-m}$. Looking at (4.10) and the Neumann boundary condition (only depends on s via the direction of the normal vector, which only depends on which piece of the boundary $\mathbf{s}$ is on; this can be determined uniquely via $\Gamma(\mathbf{s})$ by taking a limiting sequence in $\mathbf{s}$ to the boundary), we can see that under this new parametrization, $g$ only depends on $\mathbf{s}$ 
through the first $m$ components. Hence, if $\mathbf{s}_{1}, \mathbf{s}_{2} \in \overline{\mathcal{A}}$ are such that $\Gamma\left(\mathbf{s}_{1}\right)=\Gamma\left(\mathbf{s}_{2}\right)$, then $g\left(\mathbf{s}_{1}\right)=g\left(\mathbf{s}_{2}\right)$ and $(I)$ is proved.

\section{C.6 Proof of Lemma 4.5.3:}

The lower bound is attained by the strategy that immediately liquidates and then does nothing until time $T$. For the upper bound we have

$$
\begin{aligned}
\Phi \leq & \left.\sup _{(\boldsymbol{\delta}, \tau) \in \mathcal{U}} \mathbb{E}_{t}\left[X_{T}+\mathbf{q}_{T} \cdot \mathbf{S}_{T}-\phi \int_{t}^{T} \ell\left(\mathbf{q}_{u}\right) d u\right]\right|_{\mathbf{q}=0}+\mathbf{q} \cdot \mathbf{s}+\|\mathbf{q}\|_{1} \sum_{i} \xi_{i} \\
\leq & \left.\sup _{(\delta, \tau) \in \mathcal{U}^{\prime}} \mathbb{E}_{t}\left[X_{T}+\mathbf{q}_{T} \cdot \mathbf{S}_{T}-\phi \int_{t}^{T} \ell\left(\mathbf{q}_{u}\right) d u\right]\right|_{\mathbf{q}=0}+\mathbf{q} \cdot \mathbf{s} \\
& +(T-t)\left(\sum_{i} \xi_{i}+\sup _{\delta, i, \pm} \delta h(\delta)\right)+\|\mathbf{q}\|_{1} \sum_{i} \xi_{i} \\
= & +\sum_{i} \xi_{i} \sup _{\omega, t, i}\left(\mathbf{q}_{t} \cdot e_{i}\right) c_{3}(\sigma) \sqrt{T-t} \mathbb{E}\left[\chi_{1}\right]+(T-t) \sum_{i} \xi_{i} \sup _{\delta, i, \pm} \delta h(\delta) \\
& +\mathbf{q} \cdot \mathbf{s}+(T-t)\left(\sum_{i} \xi_{i}+\sup _{\delta, i, \pm} \delta h(\delta)\right)+\|\mathbf{q}\|_{1} \sum_{i} \xi_{i}
\end{aligned}
$$

where $\chi_{u}$ is the maximum (in absolute value) of a standard $n$-dimensional Brownian motion up to time $u$ and $\mathcal{U}^{\prime} \subset \mathcal{U}$ is the subset of admissible controls with $\boldsymbol{\delta}=\infty$ whenever $\|\mathbf{q}\|=0$. Finally, the result follows from Assumption 4.2.5.

Although this upper bound is crude, it is all that is necessary to continue. We now turn our attention to each term in the upper bound in this proof. The terms $\mathbf{q} \cdot \mathbf{s}+\|\mathbf{q}\|_{1} \sum_{i} \xi_{i}$ correspond to free liquidation to $\mathbf{q}=0$ with an added bonus of $\sum_{i} \xi_{i}$ for unit of each open position. There is no way for the HFT to do better than this (or even come close) on an expected value basis. The term $(T-t)\left(\sum_{i} \xi_{i}+\sup _{\omega, t, i, \pm} \delta h(\delta)\right)$ is an upper bound on the profit of all future open positions that use filled limit orders. Lastly, we must consider all market order initiated open positions (for the case where $\mathbf{q}=0$ is not an element of the continuation region). If these positions are aided with filled limit orders, then we have already accounted for such events. Otherwise, in order to produce a profit, the brownian motion must travel a certain distance from where the open position was initiated. The total number of times this happens is proportional to $\sqrt{T-t}$, and $\phi>0$ guarantees that $\mathbf{q}_{t}(\omega)$ is uniformly bounded. We are able to bound the expected round 
trip profit because the dimensionally reduced no-arbitrage region is bounded.

\section{C.7 Proof of Proposition 4.5.4 (Monotonicity):}

Let $\varphi_{1} \leq \varphi_{2}, Q \in \mathbb{N}$, and $d s>0$. Monotonicity of the operator $\mathcal{M}$ is obvious. Because $\varphi$ and its derivatives are continuous, and the domain of $\varphi$ is compact, there exists finite constants $A_{1}, A_{2}>0$ such that

$$
e^{\kappa \Delta \varphi_{2}}-e^{\kappa \Delta \varphi_{1}} \geq\left(e^{-\kappa \varphi_{2}}-e^{-\kappa \varphi_{1}}\right) e^{\kappa \mathbb{S} \varphi_{1}}=\left(e^{-\kappa\left(\varphi_{2}-\varphi_{1}\right)}-1\right) e^{\kappa \Delta \varphi_{1}} \geq-A_{1}\left(\varphi_{2}-\varphi_{1}\right)
$$

where $A_{1}$ does not depend on $\varphi_{1}$ or $\varphi_{2}, \mathbb{S}$ is the shift operator associated with $\Delta$, and

$$
\hat{\mathcal{T}}_{i j}^{d t, d s, Q}\left(t, \mathbf{s}, \mathbf{q}, \varphi_{2}\right)-\hat{\mathcal{T}}_{i j}^{d t, d s, Q}\left(t, \mathbf{s}, \mathbf{q}, \varphi_{1}\right) \geq-A_{2}\left(\varphi_{2}-\varphi_{1}\right)
$$

where $A_{2}$ only depends on $d s$.

Using (C.7) and (C.8) gives

$$
\begin{aligned}
& \mathcal{T}^{d t, d s, Q}\left(t, \mathbf{s}, \mathbf{q}, \varphi_{2}\right)-\mathcal{T}^{d t, d s, Q}\left(t, \mathbf{s}, \mathbf{q}, \varphi_{1}\right) \\
&= \varphi_{2}-\varphi_{1}+d t \frac{1}{2} \sum_{i, j=1}^{n}\left[\boldsymbol{\sigma} \boldsymbol{\sigma}^{\prime}\right]_{i j}\left(\hat{\mathcal{T}}_{i j}^{d t, d s, Q}\left(\cdot, \varphi_{2}\right)-\hat{\mathcal{T}}_{i j}^{d t, d s, Q}\left(\cdot, \varphi_{1}\right)\right) \\
& \quad+d t \sum_{i, \pm} \frac{\lambda}{\kappa} e^{-1+\kappa \xi / 2}\left(e^{\kappa \Delta \varphi_{2}}-e^{\kappa \Delta \varphi_{1}}\right) \\
& \geq\left(\varphi_{2}-\varphi_{1}\right)\left[1-d t\left\{A_{1} \sum_{i, \pm} \frac{\lambda}{\kappa} e^{-1+\kappa \xi / 2}+\frac{A_{2}}{2} \sum_{i, j=1}^{n}\left[\boldsymbol{\sigma} \boldsymbol{\sigma}^{\prime}\right]_{i j}\right\}\right] .
\end{aligned}
$$

Taking

$$
d t<\left(A_{1} \sum_{i, \pm} \frac{\lambda}{\kappa} e^{-1+\kappa \xi / 2}+\frac{A_{2}}{2} \sum_{i, j=1}^{n}\left[\boldsymbol{\sigma} \boldsymbol{\sigma}^{\prime}\right]_{i j}\right)^{-1}
$$

gives monotonicity of $\mathcal{T}$. 


\section{C.8 Proof of Proposition 4.5.6 (Consistency):}

Equation (4.20) follows by continuity of $\varphi$. To prove (4.19), let $\left(t^{\prime}, \mathbf{s}^{\prime}, \mathbf{q}\right) \in[0, T) \times \overline{\mathcal{A}}^{\prime} \times \mathbb{Z}^{n}$ and observe:

$$
\begin{aligned}
\frac{1}{d t}\left[\mathcal{T}^{d t, d s, Q}\left(t^{\prime}+d t, \mathbf{s}^{\prime}, \mathbf{q}, \varphi\right)-\varphi\left(t^{\prime}, \mathbf{s}^{\prime}, \mathbf{q}\right)\right] & =\frac{1}{d t}\left[\varphi\left(t^{\prime}+d t, \mathbf{s}^{\prime}, \mathbf{q}\right)-\varphi\left(t^{\prime}, \mathbf{s}^{\prime}, \mathbf{q}\right)\right]-\phi \ell(\mathbf{q}) \\
& +\frac{1}{2} \sum_{i, j=1}^{n}\left[\boldsymbol{\sigma} \boldsymbol{\sigma}^{\prime}\right]_{i j} \hat{\mathcal{T}}_{i j}^{d t, d s, Q}\left(t^{\prime}+d t, \mathbf{s}^{\prime}, \mathbf{q}, \varphi\right) \\
& +\sum_{i, \pm} \frac{\lambda}{\kappa} \exp \left\{-1+\frac{\kappa \xi}{2}+\kappa \Delta \varphi\left(t^{\prime}+d t, \mathbf{s}^{\prime}, \mathbf{q}\right)\right\}
\end{aligned}
$$

The only term that does not trivially converge is $\Delta \varphi\left(t^{\prime}+d t, \mathbf{s}^{\prime}, \mathbf{q}\right.$ ) (the other terms clearly converge since $\left.\varphi \in C_{b}^{1,2}\right)$. But $\Delta \varphi\left(t^{\prime}+d t, \mathbf{s}^{\prime}, \mathbf{q}\right)$ is also continuous in $(t, \mathbf{s})$ whenever $Q \geq\|\mathbf{q}\|_{\infty}+1$, thus proving (4.19).

Observe that we do not need to include a sequence of inventory levels due to the fact that $\mathbf{q}$ resides on the sparse grid $\mathbb{Z}^{n}$. More formally, one could only consider the limiting sequence $\left(t^{\prime}+d t, \mathbf{s}^{\prime}, \mathbf{q}^{\prime}\right) \rightarrow(t, \mathbf{s}, \mathbf{q})$ sufficiently far in the tail when $\mathbf{q}^{\prime}=\mathbf{q}$ onwards.

\section{C.9 Proof of Theorem 5.3.1}

Suppose $\lambda_{i, t_{a_{0}-}}=\theta_{i}\left(1+\delta_{i}\right)$ with $\boldsymbol{\delta} \in \mathbb{R}^{n}, 0 \leq\|\boldsymbol{\delta}\|_{\infty}<\epsilon$. Defining $\tilde{\boldsymbol{\delta}} \in \mathbb{R}^{n}$ by $\tilde{\boldsymbol{\delta}}_{i}=\delta_{i} \theta_{i}$ we have the exact value of the intensity given by

$$
\lambda_{i, t}(H)=\left[\boldsymbol{\theta}+e^{-\boldsymbol{\beta}\left(t-t_{a_{0}}\right)} \tilde{\boldsymbol{\delta}}+\int_{t_{a_{0}}}^{t} e^{-\boldsymbol{\beta}(t-u)} \boldsymbol{\eta}_{u} d \mathbf{N}_{u}(H)\right] \cdot E_{i}
$$

which is the approximated value plus a linear term in $\tilde{\boldsymbol{\delta}}$.

Similarly, the log of the true probability $\mathbb{P}\left[\tau>T_{a_{k}}(1) \mid H\right]$ also differs from the approximated probability, as given in Equation (5.11), by a term that is also linear in $\tilde{\boldsymbol{\delta}}$. We can, however, make use of this linearity to justify the asymptotic behaviour of the isolated likelihood function. 
In particular, the probability in (5.11) is off by a multiplicative factor of

$$
\begin{aligned}
\exp \left\{-\boldsymbol{\beta}^{-1}\left(\mathbb{I}_{n}-e^{-\boldsymbol{\beta}\left(t_{a_{k}}-t_{a_{0}}\right)}\right) \tilde{\boldsymbol{\delta}} \cdot \mathbf{1}_{n}\right\} & =1-\boldsymbol{\beta}^{-1}\left(\mathbb{I}_{n}-e^{-\boldsymbol{\beta}\left(t_{a_{k}}-t_{a_{0}}\right)}\right) \tilde{\boldsymbol{\delta}} \cdot \mathbf{1}_{n}+o\left(\|\tilde{\boldsymbol{\delta}}\|_{\infty}\right) \\
& =1+O\left(\|\tilde{\boldsymbol{\delta}}\|_{\infty}\right)
\end{aligned}
$$

Denote $\tilde{\boldsymbol{\lambda}}_{t}$ as the approximated value of $\boldsymbol{\lambda}_{t}$. Combining (C.10) and (C.12), we have

$$
\begin{aligned}
\mathcal{L} & =\sum_{H} \prod_{k=1}^{m} \rho_{H_{k}} \cdot\left[\tilde{\lambda} .+O\left(\|\tilde{\boldsymbol{\delta}}\|_{\infty}\right)\right] \cdot \mathbb{P}\left[\tau>T_{a_{k}}(1) \mid \tilde{\boldsymbol{\lambda}} \cdot\right]\left[1+O\left(\|\tilde{\boldsymbol{\delta}}\|_{\infty}\right)\right] \\
& =\sum_{H} \prod_{k=1}^{m} \rho_{H_{k}} \cdot \tilde{\lambda} \cdot \cdot \mathbb{P}\left[\tau>T_{a_{k}}(1) \mid \tilde{\boldsymbol{\lambda}} \cdot\right]\left[1+O\left(\|\tilde{\boldsymbol{\delta}}\|_{\infty}\right)\right] \\
& =\tilde{\mathcal{L}} \cdot\left[1+O\left(\|\tilde{\boldsymbol{\delta}}\|_{\infty}\right)\right]^{m} \\
& =\tilde{\mathcal{L}} \cdot[1+O(\epsilon)]
\end{aligned}
$$

\section{C.10 Proof of Theorem 5.3.2}

Suppose $\lambda_{i, t_{a_{0}}}=\theta_{i}\left(1+\delta_{i}\right)$ with $\boldsymbol{\delta} \in \mathbb{R}^{n}, 0 \leq\|\boldsymbol{\delta}\|_{\infty}<\epsilon$. Then, for $t>t_{a_{0}}$, the true value of $\lambda_{i, t}$ is at most the approximated value, $\tilde{\lambda}_{i, t}$, multiplied by $\left(1+\delta_{i}\right)$ (since $\boldsymbol{\beta}$ is diagonal with positive elements, we also have the necessary fact that $\left.\lambda_{i, t} \geq \tilde{\lambda}_{i, t}\right)$. That is, $\tilde{\lambda}_{i, t} \leq \lambda_{i, t} \leq \tilde{\lambda}_{i, t}\left(1+\delta_{i}\right)$. Using this knowledge, we have

$$
\begin{aligned}
& \frac{\tilde{\mathcal{L}}}{\mathcal{L}}=\frac{\tilde{\mathcal{L}}}{\sum_{H} \prod_{k=1}^{m} \rho_{H_{k}} \cdot \lambda_{T_{a_{k}}(2), t_{a_{k}}-}(H) \cdot \mathbb{P}\left[\tau>T_{a_{k}}(1) \mid H, \lambda_{t_{a_{o}}-}\right]} \\
& \geq \frac{\tilde{\mathcal{L}}}{\sum_{H} \prod_{k=1}^{m} \rho_{H_{k}} \cdot \tilde{\lambda}_{T_{a_{k}}(2), t_{a_{k}}-}(H)\left(1+\delta_{T_{a_{k}}(2)}\right) \cdot \mathbb{P}\left[\tau>T_{a_{k}}(1) \mid H, \boldsymbol{\lambda}_{t_{a_{o}}-}\right]} \\
& \geq \frac{\tilde{\mathcal{L}}}{\sum_{H} \prod_{k=1}^{m} \rho_{H_{k}} \cdot \tilde{\lambda}_{T_{a_{k}}(2), t_{a_{k}}-}(H)\left(1+\delta_{T_{a_{k}}(2)}\right) \cdot \mathbb{P}\left[\tau>T_{a_{k}}(1) \mid H, \tilde{\boldsymbol{\lambda}}_{t_{a_{o}}-}\right]}
\end{aligned}
$$




$$
\begin{aligned}
& >\frac{\tilde{\mathcal{L}}}{\sum_{H} \prod_{k=1}^{m} \rho_{H_{k}} \cdot \tilde{\lambda}_{T_{a_{k}}(2), t_{a_{k}}}(H)(1+\epsilon) \cdot \mathbb{P}\left[\tau>T_{a_{k}}(1) \mid H, \tilde{\boldsymbol{\lambda}}_{t_{a_{o}}-}\right]} \\
& =(1+\epsilon)^{-m}
\end{aligned}
$$

where the second inequality is due the fact that $\boldsymbol{\beta}^{-1}\left(\mathbb{I}_{n}-e^{-\boldsymbol{\beta} t}\right) \boldsymbol{\delta} \cdot \mathbf{1}_{n}>0$ when $\boldsymbol{\beta}$ is diagonal.

Similarly,

$$
\begin{aligned}
\frac{\tilde{\mathcal{L}}}{\mathcal{L}} & =\frac{\tilde{\mathcal{L}}}{\sum_{H} \prod_{k=1}^{m} \rho_{H_{k}} \cdot \lambda_{T_{a_{k}}(2), t_{a_{k}}-}(H) \cdot \mathbb{P}\left[\tau>T_{a_{k}}(1) \mid H, \boldsymbol{\lambda}_{t_{a_{o}}-}\right]} \\
& \leq \frac{\tilde{\mathcal{L}}}{\sum_{H} \prod_{k=1}^{m} \rho_{H_{k}} \cdot \tilde{\lambda}_{T_{a_{k}}(2), t_{a_{k}}-}(H) \cdot \mathbb{P}\left[\tau>T_{a_{k}}(1) \mid H, \boldsymbol{\lambda}_{t_{a_{o}}-}\right]} \\
& <\frac{\tilde{\mathcal{L}}}{\sum_{H} \prod_{k=1}^{m} \rho_{H_{k}} \cdot \tilde{\lambda}_{T_{a_{k}}(2), t_{a_{k}}-}(H) \mathbb{P}\left[\tau>T_{a_{k}}(1) \mid H, \tilde{\boldsymbol{\lambda}}_{t_{a_{o}}}\right] \exp \left\{-\boldsymbol{\beta}^{-1}\left(\mathbb{I}_{n}-e^{-\boldsymbol{\beta} T_{a_{k}}(1)}\right) \mathbf{1}_{n} \cdot \mathbf{1}_{n}\|\boldsymbol{\theta}\| \epsilon\right\}} \\
& <\frac{\tilde{\mathcal{L}}}{\sum_{H} \prod_{k=1}^{m} \rho_{H_{k}} \cdot \tilde{\lambda}_{T_{a_{k}}(2), t_{a_{k}}-}(H) \cdot \mathbb{P}\left[\tau>T_{a_{k}}(1) \mid H, \tilde{\boldsymbol{\lambda}}_{t_{a_{o}}-}\right] \cdot \exp \left\{-\boldsymbol{\beta}^{-1} \mathbf{1}_{n} \cdot \mathbf{1}_{n}\|\boldsymbol{\theta}\| \epsilon\right\}} \\
& =\exp \left\{m \epsilon\|\boldsymbol{\theta}\| \sum_{j=1}^{n} \beta_{j j}^{-1}\right\}
\end{aligned}
$$

Taking the logarithm of both inequalities yields the result. 


\section{Appendix D}

\section{Some Explicit Formulae}

\section{D.1 Exact Optimal Trading Strategy in the Single Asset Hawkes Framework}

Although an exact optimal control is not analytically tractable in general, the feedback control form for the cases of exponential and power-law FPs can be obtained within our modeling framework.

Proposition D.1.1. Exact Optimal Controls for Exponential and Power-Law. Suppose that the scale parameter process $\kappa_{t}^{ \pm}$is bounded away from zero almost surely. More specifically, assume that $\mathbb{P}\left[\inf _{t \in[0, T]} \kappa_{t}^{ \pm}>0\right]=1$.

1. If $h^{ \pm}(\delta ; \boldsymbol{\kappa})=e^{-\kappa^{ \pm} \delta}$ for $\delta>0$, then the feedback control of the optimal trading strategy for the HJB equation (3.7) is given by

$$
\delta_{t}^{ \pm}=\max \left\{\frac{1}{\kappa^{ \pm}}-\left\{\mathbb{S}_{q \lambda}^{ \pm} g-\mathbb{S}_{\lambda}^{ \pm} g\right\}, 0\right\}
$$

2. If $h^{ \pm}(\delta ; \boldsymbol{\kappa})=\left(1+\kappa^{ \pm} \delta\right)^{\gamma^{ \pm}}$for $\delta>0$, then the feedback control of the optimal trading strategy for the HJB equation (3.7) is given by

$$
\delta_{t}^{ \pm}=\max \left\{\frac{\alpha}{\alpha-1}\left(\frac{1}{\kappa^{ \pm}}-\left\{\mathbb{S}_{q \lambda}^{ \pm} g-\mathbb{S}_{\lambda}^{ \pm} g\right\}\right), 0\right\}
$$


Here, the ansatz $\Phi=x+q s+g(t, q, \alpha, \boldsymbol{\lambda}, \boldsymbol{\kappa})$ with boundary condition $g(T, \cdot)=0$ has been applied. Furthermore, the solutions in (D.1a) and (D.1b) are unique.

Proof. Applying the first order conditions to the supremum terms and using the specified ansatz leads, after some simplifications, to the stated result. We show this in detail for $\delta^{-}$in (D.1a) only as the other cases are analogous. The relevant supremum term in the HJB equation in (3.7) simplifies to, after applying the ansatz $\Phi=x+q s+g(t, q, \alpha, \boldsymbol{\lambda}, \boldsymbol{\kappa})$,

$$
e^{-\delta^{-} \kappa^{-}}\left[\mathbb{S}_{\lambda}^{-} g-g+\delta^{-}\right]+\left(1-e^{-\delta^{-} \kappa^{-}}\right)\left[\mathbb{S}_{\lambda}^{-} g-g\right] .
$$

Differentiating (D.2) with respect to $\delta^{-}$and setting the resulting expression equal to zero yields (D.1a). Checking the second derivative of (D.2) verifies that this point is in fact a local maximum. If this point is negative, then the optimal $\delta^{-}$is $\delta^{-}=0$ by Assumption 3.3.1.

Uniqueness is trivial.

\section{D.2 Explicit Computation of $b_{\varepsilon}$}

Rather than computing $b_{\varepsilon}$ directly, it is more convenient to compute the expected integrated drift and then identify the appropriate terms. To this end we have the following result.

Proposition D.2.1. Expected Integrated Drift. The expected integrated drift is given by the expression

$$
\mathbb{E}\left[\int_{t}^{T} \alpha_{s} d s \mid \boldsymbol{\lambda}_{t}=\boldsymbol{\lambda}, \alpha_{t}=\alpha\right]=\varepsilon b_{\epsilon}(t, \boldsymbol{\lambda})+\alpha b_{\alpha}(t)
$$

where $\varepsilon b_{\varepsilon}(t, \boldsymbol{\lambda})=A(t)+\boldsymbol{\lambda} \cdot \mathbf{C}(t)$ and

$$
\begin{aligned}
A(t)= & \frac{\varepsilon}{\zeta}\left(\mu^{+} \tilde{\mathfrak{a}}^{+}-\mu^{-} \tilde{\mathfrak{a}}^{-}\right)\left((T-t)-b_{\alpha}(t)\right)+\boldsymbol{\zeta}^{\prime} \cdot \mathbf{B}(t), \\
\mathbf{B}(t)= & \frac{\rho}{\zeta}\left\{\mathbf{A}^{-1}\left((T-t) \mathbf{I}-\mathbf{A}^{-1}\left(\mathbf{I}-e^{-\mathbf{A}(T-t)}\right)\right)\right. \\
& \left.\quad-(\mathbf{A}-\zeta \mathbf{I})^{-1}\left(b_{\alpha}(t) \mathbf{I}-\mathbf{A}^{-1}\left(\mathbf{I}-e^{-\mathbf{A}(T-t)}\right)\right)\right\} \varepsilon \mathfrak{a}, \\
\mathbf{C}(t)= & \frac{\rho}{\zeta}\left\{\mathbf{A}^{-1}\left(\mathbf{I}-e^{-\mathbf{A}(T-t)}\right)-(\mathbf{A}-\zeta \mathbf{I})^{-1}\left(e^{-\zeta(T-t)} \mathbf{I}-e^{-\mathbf{A}(T-t)}\right)\right\} \varepsilon \mathfrak{a} .
\end{aligned}
$$

Lastly, $\mathfrak{a}=\left(-\mathfrak{a}^{-}, \mathfrak{a}^{+}\right)^{\prime}$ and $\boldsymbol{\zeta}=\left(\beta \theta+\mu^{+} \widetilde{\nu}+\mu^{-} \widetilde{\eta}, \beta \theta+\mu^{+} \widetilde{\eta}+\mu^{-} \widetilde{\nu}\right)^{\prime}$. 
Proof. Denoting $f(t, \alpha, \boldsymbol{\lambda})=\mathbb{E}\left[\int_{t}^{T} \alpha_{s} d s \mid \boldsymbol{\lambda}_{t}=\boldsymbol{\lambda}, \alpha_{t}=\alpha\right]$, we have, through a FeynmanKac argument, that $f$ satisfies the $\mathrm{PDE}$

$$
\left(\partial_{t}+\mathcal{L}\right) f+\lambda^{+}\left(\mathbb{S}_{\lambda}^{+} f-f\right)+\lambda^{-}\left(\mathbb{S}_{\lambda}^{-} f-f\right)+\alpha=0,
$$

where the infinitesimal generator of $\alpha$ and $\boldsymbol{\lambda}$ is

$$
\mathcal{L}=\beta\left(\theta-\lambda^{-}\right) \partial_{\lambda^{-}}+\beta\left(\theta-\lambda^{+}\right) \partial_{\lambda^{+}}-\zeta \alpha \partial_{\alpha}+\frac{1}{2} \sigma^{2} \partial_{\alpha \alpha}+\mu^{-}\left(\widetilde{\mathcal{S}}_{\lambda}^{-}-1\right)+\mu^{+}\left(\widetilde{\mathcal{S}}_{\lambda}^{+}-1\right) .
$$

Substituting the affine ansatz $f=A(t)+\boldsymbol{\lambda} \mathbf{C}(t)+\alpha b_{\alpha}(t)$ into the PDE, subject to the boundary conditions $A(T)=\mathbf{C}(T)=0$, leads to the system of coupled ODEs:

$$
\left\{\begin{aligned}
\partial_{t} A(t)+\varepsilon\left(\mu^{+} \widetilde{\mathfrak{a}}^{+}-\mu^{-} \widetilde{\mathfrak{a}}^{-}\right) b_{\alpha}(t)+\boldsymbol{\zeta}^{\prime} \cdot \mathbf{C}(t) & =0, \\
\partial_{t} \mathbf{C}(t)-\mathbf{A C}(t)+\rho b_{\alpha}(t) \varepsilon \mathfrak{a} & =0 .
\end{aligned}\right.
$$

The solution of this coupled system is given by (D.4). The assertion that $A(t)+\boldsymbol{\lambda} \cdot \mathbf{C}(t)=$ $\varepsilon b_{\varepsilon}$ with $b_{\varepsilon}$ provided in (3.15b) can be confirmed by (i) writing down the PDE which the function $b_{\varepsilon}$ satisfies, (ii) noting that it admits an affine ansatz $A_{\varepsilon}(t)+\boldsymbol{\lambda} \cdot \mathbf{C}_{\varepsilon}(t)$, and (iii) noting that the ODEs that $A_{\varepsilon}(t)$ and $\mathbf{C}_{\varepsilon}(t)$ satisfy are the same ODEs as $A(t)$ and $\mathbf{C}(t)$ with the same boundary conditions. Uniqueness then implies that they are equal.

\section{D.3 Conditional Mean of LOB Depth Process}

Lemma D.3.1. Conditional Mean of $\boldsymbol{\kappa}_{t}$. Under the dynamics given in (3.3), the conditional mean $\tilde{m}_{t}^{ \pm}(u):=\mathbb{E}\left[\kappa_{u}^{ \pm} \mid \mathcal{F}_{t}\right]$ is

$$
\tilde{m}_{t}^{ \pm}(u)=\theta_{\kappa}+\frac{\rho}{\beta_{\kappa}}\left[\eta_{\kappa} m_{t}^{ \pm}(u)+\nu_{\kappa} m_{t}^{\mp}(u)\right]+\left[\kappa_{t}^{ \pm}-\theta_{\kappa}-\frac{\rho}{\beta_{\kappa}}\left(\eta_{\kappa} \lambda_{t}^{ \pm}+\nu_{\kappa} \lambda_{t}^{\mp}\right)\right] e^{-\beta_{\kappa}(u-t)}
$$

where $m_{t}^{ \pm}(u)$ are given in Equation (C.2).

Proof. Proceeding as in the proof of Lemma 3.2.2 in Appendix C.1, $\tilde{m}_{t}^{ \pm}(u)$ satisfies the (uncoupled) system of ODEs

$$
\frac{d \tilde{m}_{t}^{ \pm}(u)}{d u}+\beta_{\kappa} \tilde{m}_{t}^{ \pm}(u)=\beta_{\kappa} \theta_{\kappa}+\rho\left[\eta_{\kappa} m_{t}^{ \pm}(u)+\nu_{\kappa} m_{t}^{\mp}(u)\right]
$$

where $m_{t}^{ \pm}(u)$ is given by Equation (C.2). Solving (D.8) with the initial condition $\tilde{m}_{t}^{ \pm}(t)=$ $\kappa_{t}^{ \pm}$gives the stated result. 


\section{Appendix E}

\section{Numerical Procedures}

\section{E.1 Fitting the Classical Hawkes Model to Level I Trade Data}

When all market orders are influential (i.e. when $\rho=1$ ), the path of the intensity process is fully specified. Consequently, the likelihood can be written explicitly and a straightforward maximum likelihood estimation can be used (albeit it must be maximized numerically). We appeal to Ogata (1978) for results concerning consistency and asymptotic convexity of the likelihood function.

Suppose $\left\{t_{1}, t_{2}, \ldots, t_{n}\right\}$ is a set of observed trade times (with $t_{n} \leq T$ representing the time of the last trade) and $\left\{B_{1}, B_{2}, \ldots, B_{n}\right\}$ are buy/sell indicators (i.e. 0 if the trade is a market sell and 1 if the trade is a market buy). Then the hazard rates and their integrals at an arbitrary time $t$ can be found by integrating (3.2) and are explicitly given by

$$
\lambda_{t}^{ \pm}=\theta+\sum_{i=1}^{n} H_{i}^{ \pm} e^{-\beta\left(t-t_{i}\right)} \quad \text { and } \quad \int_{0}^{t} \lambda_{u}^{ \pm} d u=\theta t+\sum_{i=1}^{n} H_{i}^{ \pm} \frac{1-e^{-\beta\left(t-t_{i}\right)}}{\beta}
$$

where $H_{i}^{ \pm}=\left(B_{i} \eta+\left(1-B_{i}\right) \nu, B_{i} \nu+\left(1-B_{i}\right) \eta\right)$. Finally, the log-likelihood

$$
\mathcal{L}=-2 \theta T+\sum_{i=1}^{n}\left\{B_{i} \log \left(\lambda_{t_{i}}^{+}\right)+\left(1-B_{i}\right) \log \left(\lambda_{t_{i}}^{-}\right)-(\eta+\nu) \frac{1-e^{-\beta\left(T-t_{i}\right)}}{\beta}\right\} .
$$

Maximizing this log-likelihood results in the MLEs of the model parameters, and substi- 
tution back into Equation (E.1) provides the estimated path of activity. Integrating this activity over the last second, i.e., $\int_{t-1}^{t} \lambda_{u}^{ \pm} d u$, provides us with a smoothed version of the intensity and shown in Figure 3.1 as the path labeled "Fitted." In turn, this is directly comparable to the one second historical intensity in Figure 3.1 labeled as "Historical."

For the time window 3:30pm to 4:00pm on Feb 1, 2008 for IBM, the estimated parameters are as follows:

$$
\widehat{\beta}=180.05, \quad \widehat{\theta}=2.16, \quad \widehat{\eta}=64.16, \quad \text { and } \quad \widehat{\nu}=55.74
$$

Notice that the spikes in the historical intensity are often above the fitted intensities. The reason for this difference is that, in this calibration, the fitted intensities assume that all trades are influential (i.e. $\rho=1$ ). Consequently, the size of the jump in intensities must be smaller than the true jump size to preserve total mean activity of trades. When a full calibration is carried out, in which $\rho$ is not necessarily 1 and the influential/non-influential nature of the event must be filtered, the jump sizes are indeed larger.

\section{E.2 Simulation Procedure for Section 3.6}

Here we describe in more detail the approach to simulating the PnL distribution of the HF strategy. Note that this method produces an exact simulation; specifically, there are no discretization errors that would be associated with approximating a continuous time process by a discrete one (i.e., simulated interarrival times are correct up to machine precision).

1. Generate the duration until the next market order given the current level of activity $\lambda_{t_{n}}^{ \pm}$.

- In between orders, the total rate of order arrival is $\lambda_{t}=2 \theta+\left(\lambda_{t_{n}}^{+}+\lambda_{t_{n}}^{-}-\right.$ $2 \theta) e^{-\beta\left(t-t_{n}\right)}$. To obtain a random draw of the time of the next trade, draw a uniform $u \sim U(0,1)$ and find the root of the equation $\tau e^{\tau}=\frac{1}{2 \theta}\left(\lambda_{t_{n}}-2 \theta\right) e^{\varsigma}$ where $\varsigma=\frac{\lambda_{t_{n}}-2 \theta}{2 \theta}+\frac{\beta}{2 \theta} \ln u .{ }^{69}$ Then, $T_{n+1}=\frac{1}{\beta}(\tau-\varsigma)$ is a sample for the next duration and $t_{n+1}=t_{n}+T_{n+1}$.

2. Decide if the trade is a buy or sell market order.

- The probability that the MO is a buy order is $p_{b u y}=\frac{\theta+\left(\lambda_{t_{n}}^{+}-\theta\right) e^{-\beta T_{n+1}}}{2 \theta+\left(\lambda_{t_{n}}^{+}+\lambda_{t_{n}}^{-}-2 \theta\right) e^{-\beta T_{n+1}}}$. Therefore, draw a uniform $u \sim U(0,1)$, and if $u<p_{\text {buy }}$, the order is a buy order; otherwise it is a sell order.

\footnotetext{
${ }^{69}$ This can be efficiently computed using the Lambert-W function.
} 
- Set the buy/sell indicator $B_{n+1}=1$ if it is a buy market order and $B_{n+1}=-1$ if it is a sell market order.

3. Decide whether the market order filled the agent's posted limit order.

- Compute the posted limit order at the time of the market order $\lambda_{t_{n+1}}^{ \pm}=\theta+$ $\left(\lambda_{t_{n}}^{ \pm}-\theta\right) e^{-\beta T_{n+1}}$.

- Draw a uniform $u \sim U(0,1)$.

- If the market order was a sell (buy) order, then if $u<e^{-\kappa_{t}^{-} \delta_{t}^{-}}\left(u<e^{-\kappa_{t}^{+} \delta_{t}^{+}}\right)$the agent's buy (sell) limit order was lifted (hit).

4. Update the midprice and drift of the asset.

- Generate two correlated normals $Z_{1}$ and $Z_{2}$ with zero mean and covariances:

$$
\begin{aligned}
& \mathbb{C}\left(Z_{1}, Z_{1}\right)=\frac{\sigma^{2}}{\zeta^{2}}\left(T_{n+1}-2 \frac{1-e^{-\zeta T_{n+1}}}{\zeta}+\frac{1-e^{-2 \zeta T_{n+1}}}{2 \zeta}\right), \\
& \mathbb{C}\left(Z_{2}, Z_{2}\right)=\frac{\sigma^{2}}{2 \zeta}\left(1-e^{-2 \zeta T_{n+1}}\right) \\
& \mathbb{C}\left(Z_{1}, Z_{2}\right)=\frac{\sigma^{2}}{2 \zeta^{2}}\left(1-2 e^{-\zeta T_{n+1}}+e^{-2 \zeta T_{n+1}}\right) .
\end{aligned}
$$

Generate a third independent standard normal $Z$.

- Update price and drift. $S_{t_{n+1}}=S_{t_{n}}+\alpha_{t_{n}} \frac{1}{\zeta}\left(1-e^{-\zeta_{n+1}}\right)+Z_{1}+\sigma \sqrt{T_{n+1}} Z$ and $\alpha_{t_{n+1}}=e^{-\zeta T_{n+1}} \alpha_{t_{n}}+Z_{2}$.

5. Update the inventory and agent's cash:

$$
X_{t_{n+1}}=X_{t_{n}}+B_{n+1} S_{t_{n+1}}+\delta_{t_{n+1}}^{+} \cdot \mathbf{1}_{B_{n+1}=1}+\delta_{t_{n+1}}^{-} \cdot \mathbf{1}_{B_{n+1}=-1}
$$

and $q_{t_{n+1}}=q_{t_{n}}-B_{n+1}$.

6. Decide if the trade is influential, and update activities, FPs, and drift.

- Draw a uniform $u \sim(0,1)$; if $u<\rho$, the trade is influential, so set $H_{n+1}=1$; otherwise set $H_{n+1}=0$. Finally,

$$
\begin{aligned}
& \lambda_{t_{n+1}}^{ \pm}=\theta+\left(\lambda_{t_{n}}^{ \pm}-\theta\right) e^{-\beta T_{n+1}}+\left(\frac{1}{2}\left(1 \pm B_{n+1}\right) \nu+\frac{1}{2}\left(1 \mp B_{n+1}\right) \eta\right) H_{n+1}, \\
& \kappa_{t_{n+1}}^{ \pm}=\theta_{\kappa}+\left(\kappa_{t_{n}}^{ \pm}-\theta_{\kappa}\right) e^{-\beta_{\kappa} T_{n+1}}+\left(\frac{1}{2}\left(1 \pm B_{n+1}\right) \nu_{\kappa}+\frac{1}{2}\left(1 \mp B_{n+1}\right) \eta_{\kappa}\right) H_{n+1}, \\
& \alpha_{t_{n+1}}=\frac{1}{2}\left(1+B_{n+1}\right) \epsilon^{+}-\frac{1}{2}\left(1-B_{n+1}\right) \epsilon^{-}+\alpha_{t_{n+1}}
\end{aligned}
$$

7. Repeat from Step 1 until $t_{n+1} \geq T$.

8. Evolve the diffusion from the last time prior to maturity until maturity using Step 4 with $t_{n+1}=T$. 
9. Compute the terminal $\mathrm{PnL}=X_{T}+q_{T} S_{T}\left[1-c_{\text {trans }} \cdot \operatorname{sign}\left(q_{T}\right)\right]$, where $c_{\text {trans }}$ is the terminal liquidation cost.

The PnLs for the other types of HFTs employed in the simulation are obtained similarly.

\section{E.3 Evaluating Non-Trivial Terms in the Numerical Scheme of Section 4.5.2}

This section illustrates how to apply the dimensional reduction to terms where the result is non-trivial. All expressions are given explicitly for the $n=2$ example. In what follows, all functions are evaluated at a fixed inventory level, $\mathbf{q}$, and this dependence is supressed.

\section{E.3.1 The Mixed Partial Term}

For $i \neq j$, we numerically approximate the following term that appears in (4.12):

$$
\frac{\partial^{2} g}{\partial s_{i} \partial s_{j}} \approx \frac{g\left(s_{i}+d \tilde{s}, s_{j}+d \tilde{s}, \cdot\right)-g\left(s_{i}+d \tilde{s}, \cdot\right)-g\left(s_{j}+d \tilde{s}, \cdot\right)+g(\cdot)}{d \tilde{s}^{2}}
$$

where $d \tilde{s}$ is the step size of the finite difference approximation. Note that if we want the grids to line up in our $n=2$ example, we require $d \tilde{s}=d s$ where $d s$ is the step size on the dimensionally reduced grid given in Appendix E.3.3.

\section{E.3.2 Dimensional Reduction}

Recall that Corollary 4.5.2 states that there exists $v \in \mathbb{R}^{n} \backslash\{0\}$ such that $D_{v} g=0$. In our example, $v=\mathbf{1}$, so we take $\mathbf{s}:=s_{1}-s_{2}$.

Continuing from (E.3), we then have

$$
\frac{\partial^{2} g}{\partial s_{1} \partial s_{2}} \approx \frac{2 g(\cdot)-g(\mathbf{s}-d s, \cdot)-g(\mathbf{s}+d s, \cdot)}{d s^{2}}
$$

where the right hand side is defined on the dimensionally reduced grid and taken at the previous time step $(t+d t)$. 
For the 2-dimensional illustrative example studied in Chapter 4, the full numerical approximation for the diffusive term in $\mathcal{L} g$ is then given by

$$
\frac{g(\mathbf{s}+d s, t+d t)+g(\mathbf{s}-d s, t+d t)-2 g(\mathbf{s}, t+d t)}{2 d s^{2}}\left(\left[\boldsymbol{\sigma} \boldsymbol{\sigma}^{\prime}\right]_{11}+\left[\boldsymbol{\sigma} \boldsymbol{\sigma}^{\prime}\right]_{22}-2\left[\boldsymbol{\sigma} \boldsymbol{\sigma}^{\prime}\right]_{12}\right) .
$$

\section{E.3.3 The Neumann Condition}

For $\mathbf{s} \in \partial \mathcal{A}$, numerically impose $(\mathbf{q}+\nabla g) \cdot \boldsymbol{n}=0$. Take $d \bar{s}=c_{0} d s$ so the grids match up, where $c_{0}$ depends only on the choice of orthogonal basis of $B$.

In our $n=2$ example, we have the normal vector $\boldsymbol{n}=(1,-1)$ and $c_{0}=1$ resulting

in $\nabla g \approx \mp\left(\frac{g(\mathbf{s})-g(\mathbf{s} \pm d s)}{d s}, \frac{g(\mathbf{s} \pm d s)-g(\mathbf{s})}{d s}\right)$ with the upper (lower) symbol for reflection on the upper (lower) boundary when viewed graphically. The relation is only approximate because we are using a finite difference method. Finally, for $\mathbf{s} \in \partial \mathcal{A}$, we have

$$
g(\mathbf{s}) \approx g(\mathbf{s} \pm d s) \pm \frac{\left(q_{1}-q_{2}\right) d s}{2} .
$$

\section{E.3.4 Explicit Example when $n=3$}

The analogue of the right-most coefficient in (E.5) is given by

$$
\left[\boldsymbol{\sigma} \boldsymbol{\sigma}^{\prime}\right]_{11}+\left[\boldsymbol{\sigma} \boldsymbol{\sigma}^{\prime}\right]_{22}+\left[\boldsymbol{\sigma} \boldsymbol{\sigma}^{\prime}\right]_{33}-2\left[\boldsymbol{\sigma} \boldsymbol{\sigma}^{\prime}\right]_{12}-2\left[\boldsymbol{\sigma} \boldsymbol{\sigma}^{\prime}\right]_{13}+2\left[\boldsymbol{\sigma} \boldsymbol{\sigma}^{\prime}\right]_{23}
$$

and, for $\mathbf{s} \in \partial \mathcal{A}$, we have

$$
g(\mathbf{s}) \approx g(\mathbf{s} \pm d s) \pm \frac{\left(q_{1}-q_{2}-q_{3}\right) d s}{3} .
$$

\section{E.4 Computing the Posterior Distribution of $\lambda_{t}$}

To compute the posterior probabilities in a cluster of size $m$, we simply apply the Bayes Rule since we know the prior probability of each possible intensity path. In particular, for each potential path, we compute the likelihood of the data given an $H$ (jump type indicator) sequence, multiply the likelihood by the prior probability for $H_{i}$ (denoted $q_{i}$ 
for the $i^{\text {th }}$ path), and then normalize these quantities so that they sum to 1 . That is,

$$
\mathbb{P}\left[\boldsymbol{\lambda}_{t}=\boldsymbol{\lambda}^{(i)} \mid \overline{\mathcal{F}}_{t}\right] \approx \mathbb{P}\left[\boldsymbol{\lambda}_{t}=\boldsymbol{\lambda}^{(i)} \mid \sigma\left(\left\{\mathbf{N}_{u}\right\}_{u \in\left[t_{k_{0}}, t\right]}\right), \boldsymbol{\lambda}_{t_{k_{0}}}=\boldsymbol{\theta}\right]=\frac{q_{i} \hat{\mathcal{L}}_{i}}{\sum_{j} q_{j} \hat{\mathcal{L}}_{j}}
$$

$$
\text { with } \quad \hat{\mathcal{L}}_{i}=\tilde{\mathcal{L}}\left(H_{i}\right) \cdot \mathbb{P}\left[T>T_{a_{m+1}}(1) \mid H_{i}\right]
$$

and $\tilde{\mathcal{L}}(H)$ given by Equation (5.9). This is analogous to the Isolated Likelihood function given in (5.9), except it contains an extra survival probability term to account for no events since the last arrival. 


\section{Bibliography}

Alfonsi, A. and A. Fruth (2010). Optimal execution strategies in limit order books with general shape function. Journal of Quantitative Finance 10(2), 143-157.

Alfonsi, A., A. Schied, and A. Slynko (2012). Order book resilience, price manipulation, and the positive portfolio problem. SIAM Journal on Financial Mathematics 3, 511533.

Almgren, R. (2003, January). Optimal execution with nonlinear impact functions and trading-enhanced risk. Applied Mathematical Finance 10(1), 1-18.

Almgren, R. (2012). Optimal trading with stochastic liquidity and volatility. SIAM Journal on Financial Mathematics 3, 163-181.

Almgren, R. and N. Chriss (2000). Optimal execution of portfolio transactions. Journal of Risk 3.

Avellaneda, M. and S. Stoikov (2008, November). High-frequency trading in a limit order book. Quantitative Finance 8, 217-224.

Azizpour, S. and K. Giesecke (2008). Self-exciting corporate defaults: Contagion vs. frailty. Available at http://arxiv.org/pdf/1104.1773.

Bacry, E. and J. F. Muzy (2013). Hawkes model for price and trades high-frequency dynamics. Available at http://arxiv.org/pdf/1301.1135v1.

Barles, G. and P. Souganidis (1991). Convergence of approximation schemes for fully nonlinear second order equations. Asymptotic Analysis 4, 271-283.

Bäuerle, N. and U. Rieder (2009). MDP algorithms for portfolio optimization problems in pure jump markets. Finance and Stochastics 13, 591-611.

Bayraktar, E. and M. Ludkovski (2012). Liquidation in limit order books with controlled intensity. Mathematical Finance, http://dx.doi.org/10.1111/j.1467-9965.2012.00529.x.

Bertsekas, D. P. and S. Shreve (1978). Stochastic Optimal Control, Volume 139 of Mathematics in Science and Engineering. New York: Academic Press. 
Bertsimas, D. and A. W. Lo (1998, April). Optimal control of execution costs. Journal of Financial Markets 1(1), 1-50.

Bouchard, B., N.-M. Dang, and C.-A. Lehalle (2011). Optimal control of trading algorithms: A general impulse control approach. SIAM Journal on Financial Mathematics 2, 404-438.

Bowsher, C. G. (2007). Modelling security market events in continuous time: Intensity based, multivariate point process models. Journal of Econometrics 141(2), 876 - 912.

Caragea, P. and R. L. Smith (2007). Asymptotic properties of computationally efficient alternative estimators for a class of multivariate normal models. Journal of Multivariate Analysis 98, 1417-1440.

Carmona, R. and K. Webster (2012a). A belief-driven order book model. Available at http://citeseerx.ist.psu.edu/viewdoc/summary?doi=10.1.1.380.1304.

Carmona, R. and K. Webster (2012b). High frequency market making. Available at http://arxiv.org/abs/1210.5781.

Carr, P., H. Geman, D. B. Madan, and M. Yor (2003). Stochastic volatility for Lévy processes. Mathematical Finance 13(3), 345-382.

Cartea, Á. and S. Jaimungal (2013a). Modeling asset prices for algorithmic and high frequency trading. Applied Mathematical Finance, Forthcoming.

Cartea, Á. and S. Jaimungal (2013b). Risk metrics and fine tuning of high-frequency trading strategies. Mathematical Finance, http://dx.doi.org/10.1111/mafi.12023.

Cartea, A. and S. Jaimungal (2014). Optimal execution with limit and market orders. Working Paper. Available at http://www.ssrn.com/abstract=2397805.

Cartea, Á., S. Jaimungal, and R. Donnelly (2013). Robust market making. SSRN: http://ssrn.com/abstract=2310645.

Cartea, A., S. Jaimungal, and D. Kinzebulatov (2013). Algorithmic trading with learning. Working Paper. Available at http://www.ssrn.com/abstract=2373196.

Cartea, A., S. Jaimungal, and J. Ricci (2014). Buy low, sell high: A high-frequency trading perspective. SIAM Journal of Financial Mathematics, Forthcoming.

Cartea, Á. and J. Penalva (2012). Where is the value in high frequency trading? Quarterly Journal of Finance 2(3), 1-46.

Cebirog̃lu, G. and U. Horst (2013). Optimal order exposure and the market impact of limit orders. SSRN: http://ssrn.com/abstract=1997092. 
CFTC and SEC (2010, September). Findings regarding the market events of May 6, 2010. Report, SEC.

Chandler, R. E. and S. Bate (2007). Inference for clustered data using the independence loglikelihood. Biometrika 94, 167-183.

Chornoboy, E. S., L. P. Schramm, and A. F. Karr (1988). Maximum likelihood identification of neural point process systems. Biological Cybernetics 59, 265-275.

Cont, R., S. Stoikov, and R. Talreja (2010). A stochastic model for order book dynamics. Operations Research 58, 217-224.

Cvitanic, J. and A. A. Kirilenko (2010). High Frequency Traders and Asset Prices. SSRN eLibrary.

Daley, D. and D. Vere-Jones (2007). An Introduction to the Theory of Point Processes: Volume II: General Theory and Structure. Springer.

Doucet, A., S. Godsill, and C. Andrieu (2000). On sequential Monte Carlo sampling methods for Bayesian filtering. Statistics and Computing 10, 197-208.

Easley, D. and M. O'Hara (1992, June). Time and the process of security price adjustment. The Journal of Finance XLVII(2), 577-605.

Embrechts, P., T. Liniger, and L. Lin (2011). Multivariate Hawkes processes: an application to financial data. Journal of Applied Probability 48A, 367-378.

European Commission (2010). Public consultation: Review of the markets in financial instruments directive (MiFID).

Forsyth, P., S. Kennedy, J. Tse, and H. Windcliff (2012). Optimal trade execution: a mean-quadratic-variation approach. Journal of Economic Dynamics and Control 36, 1971-1991.

Frei, C. and N. Westray (2013). Optimal execution of a VWAP order: A stochastic control approach. Mathematical Finance, available at http://dx.doi.org/10.1111/mafi.12048.

Gordon, N. J., D. J. Salmond, and A. F. M. Smith (1993). Novel approach to nonlinear/non-Gaussian Bayesian state estimation. IEEE Proceedings F on Radar and Signal Processing 140(2), 107-113.

Guéant, O., C.-A. Lehalle, and J. Fernandez Tapia (2012). Optimal portfolio liquidation with limit orders. SIAM Journal on Financial Mathematics 3(1), 740-764.

Guéant, O., C.-A. Lehalle, and J. Fernandez Tapia (2013). Dealing with the inventory risk: a solution to the market making problem. Mathematics and Financial Economics 7(4), 477-507. 
Guéant, O. and G. Royer (2014). VWAP execution and guaranteed VWAP. SIAM Journal of Financial Mathematics 5(1), 445-471.

Guilbaud, F. and H. Pham (2013a). Optimal high-frequency trading in a pro rata microstructure with predictive information. Mathematical Finance (forthcoming), available at http://arxiv.org/pdf/1205.3051v1.

Guilbaud, F. and H. Pham (2013b). Optimal high frequency trading with limit and market orders. Quantitative Finance 13(1), 79-94.

Gusto, G. and S. Schbath (2005). Fado: A statistical method to detect favored or avoided distances between occurrences of motifs using the Hawkes' model. Statistical Applications in Genetics and Molecular Biology 4(1).

Hasbrouck, J. and G. Saar (2013). Low-latency trading. Journal of Financial Markets.

Hawkes, A. (1971). Spectra of some self-exciting and mutually exciting point processes. Biometrika 58(1), 83-90.

Heston, S. L. (1993). A closed-form solution for options with stochastic volatility with applications to bond and currency options. The Review of Financial Studies 6(2), $327-343$.

Ho, T. and H. R. Stoll (1981). Optimal dealer pricing under transactions and return uncertainty. Journal of Financial Economics 9, 47-73.

Horst, U. and F. Naujokat (2014). When to cross the spread: Trading in two-side limit order books. SIAM Journal of Financial Mathematics 5(1), 278-315.

Horst, U. and C. Rothe (2008). Queuing, social interactions and the microstructure of financial markets. Macroeconomic Dynamics 12, 211-233.

Hull, J. C. (2014). Options, Futures, and Other Derivatives (9 ed.). Pearson. ISBN: 978-0132164948.

Jaimungal, S. and D. Kinzebulatov (2014, July). Optimal execution with a price limiter. RISK.

Jaimungal, S., D. Kinzebulatov, and D. Rubisov (2013). Optimal accelerated share repurchase. Working Paper. Available at http://www.ssrn.com/abstract=2360394.

Kharroubi, I. and H. Pham (2010). Optimal portfolio liquidation with execution cost and risk. SIAM Journal on Financial Mathematics 1, 897-931.

Kirilenko, A. A., A. P. S. Kyle, M. Samadi, and T. Tuzun (2010). The Flash Crash: The Impact of High Frequency Trading on an Electronic Market. SSRN eLibrary. 
Kou, S. G. (2002). A jump-diffusion model for option pricing. Management Science 48(8), 1086-1101.

Large, J. (2007). Measuring the resiliency of an electronic limit order book. Journal of Financial Markets 10(1), 1-25.

Madhavan, A. (2000). Market microstructure: A survey. Available at SSRN: http://ssrn.com/abstract=218180.

Mohler, G. O., M. B. Short, P. J. Brantingham, F. P. Schoenberg, and G. E. Tita (2011). Self-exciting point process modeling of crime. Journal of the Americal Statistical Association 106, 100-108.

Obizhaeva, A. A. and J. Wang (2013). Optimal trading strategy and supply/demand dynamics. Journal of Financial Markets 16(1), 1-32.

Ogata, Y. (1978). The asymptotic behaviour of maximum likelihood estimators for stationary point processes. Ann. Inst. Statist. Math. 30, 243-261.

Ogata, Y. (1988). Statistical models for earthquake occurrences and residual analysis for point processes. Journal of the Americal Statistical Association 83, 9-27.

O’Hara, M. (1998). Market Microstructure Theory. Wiley. ISBN: 978-0631207610.

Øksendal, B. and A. Sulem (2007). Applied Stochastic Control of Jump Diffusions, Volume 2. Berlin, Heidelberg, New York: Universitext, Springer Verlag.

Pitt, M. K. and N. Shephard (1999). Filtering via simulation: Auxiliary particle filters. Journal of the American Statistical Association 94(446), 590-599.

Roşu, I. (2009). A dynamic model of the limit oder book. Review of Financial Studies 22, 4601-4641.

Sarkar, A. and R. A. Schwartz (2009). Market sidedness: Insights into motives for trade initiation. The Journal of Finance 64(1), 375-423.

Shek, H. H. S. (2011). Modeling high frequency market order dynamics using self-excited point process. Available at SSRN: http://ssrn.com/abstract=1668160.

Stein, M., Z. Chi, and L. Welty (2004). Approximating likelihoods for large spatial data sets. Journal of the Royal Statistical Society 66(2), 275-296.

The Government Office for Science, London (2012). Foresight: The future of computer trading in financial markets.

Thorarinsdottir, T. L. (2013). Calibration diagnostics for point process models via the probability integral transform. Available at http://arxiv.org/pdf/1305.3409v1. 
Toke, I. (2011). Market making in an order book model and its impact on the spread. In F. Abergel, B. Chakrabarti, A. Chakraborti, and M. Mitra (Eds.), Econophysics of Order-driven Markets, New Economic Windows, pp. 49-64. Springer Milan.

Varin, C., N. Reid, and D. Firth (2011). An overview of composite likelihood methods. Statistica Sinica 21, 5-42.

Vere-Jones, D. (1995). Forecasting earthquakes and earthquake risk. International Journal of Forecasting 11, 503-538. 